\title{
SELECTIVE COLLECTION OF FINE PARTICLES BY WATER DROPS
}

A Thesis Submitted for the Degree of

Doctor of Philosophy

By

Kathika Rashanthi Liyanaarachchi

B.Sc. Eng. (Hons)

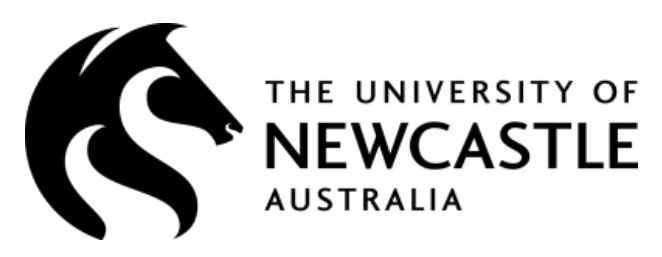

University of Newcastle, Australia. February 2015 


\section{STATEMENT OF ORIGINALITY}

This Thesis contains no material which has been accepted for the award of any other degree or diploma in any university or other tertiary institution and, to the best of my knowledge and belief, contains no material previously published or written by another person, except where due reference has been made in the text. I give the consent to this copy of my thesis, when deposited in the University Library, being made available for loan and photocopying subject to the provisions of the Copyright Act 1968.

I hereby certify that the work embodied in this thesis contains published papers/scholarly work of which I am a joint author. A complete list of these publications is included in page ix.

Kathika Rashanthi Liyanaarachchi

Professor Kevin Galvin

Principal Supervisor 
Dedicated to My Loving Husband \& My Family 


\section{ACKNOWLEDGEMENTS}

I would like to thank my supervisor, Professor Kevin Galvin for his invaluable guidance, advice and support throughout this research. I would like to extend my sincere thanks to Dr. Grant Webber for his cooperation. Special thanks go to Dr. Peter Ireland and Mr. Mark Mason for their continuous support and encouragement during this project. I am also grateful to Dr. Simon Iveson for his valuable comments on my thesis.

Many thanks to Mr. David Roberts and Mr. Bill Williams for their assistance in the construction of the laboratory experimental apparatus. The enthusiasm of the members of advanced particle processing technology group and the technical staff in the discipline of Chemical Engineering are gratefully acknowledged.

I am blessed to have a good support from my family as well as my husband throughout my $\mathrm{PhD}$. Further, I would like to thank the financial support of Australian Coal Association Research Program (ACARP) for this research. 


\section{ABSTRACT}

Fine particle processing has long been a challenge in the minerals processing industry. Ores often have to be ground to less than $100 \mu \mathrm{m}$ in size in order to liberate the valuable components. Even in the coal industry, where there is minimal comminution, a considerable fraction of the run-of-mine coal is less than $100 \mu \mathrm{m}$ in size. Hence, it can be difficult to efficiently recover these fine particles using existing beneficiation methods, such as flotation. In the past few decades, there has been on-going effort focussed on developing methods to separate finer particles in an efficient way, with an increasing interest in approaches that involve dry particles.

This Thesis is concerned with the potential for achieving selective separations through contacting particles with water drops. More specifically, the objective was to achieve selective capture of the hydrophilic particles via the water phase, leaving the hydrophobic particles in a dry state. Two different approaches were investigated through the establishment of novel experimental systems.

The first approach involved a system consisting of falling water drops passing through a gas-suspension of particles. Conceptually, this approach is the opposite of conventional flotation. A gaseous dispersion of particles was used instead of a water based dispersion of particles, and a system of falling water drops was used instead of a system of rising air bubbles. Thus the particles travelled horizontally with the airflow, colliding with drops via the gas side of the gas-liquid interface of the falling drops. The hydrophobic particles either adhered at the surface or were deflected, while the hydrophilic particles were engulfed completely, settling through to the base of the drop. Ideally the hydrophobic particles passed through the collection zone, reporting in a dry form. Thus, in the context of the coal industry, a high calorific dry product should be generated. Due to the low viscosity of air compared to water, the collision efficiency of the particles with the water drops should increase significantly compared to conventional flotation. Thus viscous forces become far less significant, allowing the inertial forces to dominate, even for ultrafine particles. Energy consumption should then be much lower. 
A new laboratory-scale experimental system was then designed and set up to further investigate this approach. The development of this facility represented a major outcome of the study, the goal being to control the steady flow of the particles and the drops to achieve reproducible and meaningful data sets. A number of design modifications were needed to achieve these requirements. The experimental system consisted of a feed column, water distribution chamber mounted over a collision chamber, underflow collection system, and dry particle cyclone stage. The airflow was driven by an air suction pump. Hydrophilic and hydrophobic particles covering a broad size range (8$116 \mu \mathrm{m})$ and spherical and irregular shapes were used in the experimental program to investigate the selective collection efficiency of this system. Results, expressed in terms of the recovery to underflow, were found to be highly reproducible, to within $3 \%$.

A simple mathematical model was also developed to describe the interception of the particles with the water drops. Particles were assumed to be collected if there was any overlap in the trajectories of the particles and the drops. In one version, the particle velocity was assumed to be much higher than the falling speed of the drops, while in a second version the particle velocity was assumed to be much smaller than the drop speed. These models were then validated using the Discrete Element Method, demonstrating the accuracy of the model in these two extremes. Moreover, the DEM approach allowed intermediate, less extreme, conditions to be examined. Thus the modelling approach provided an appreciation of the principle parameters governing the interception, most notably the water flux, droplet diameter, and active length of the collection zone. Clearly, the model did not consider the subtle effects associated with the particle-drop adhesion, dynamic wetting, disengagement from the fluid streamlines, and particle shape effects. These more subtle effects were manifested through the experimental work.

The recovery of the particles in the underflow was calculated relative to the total feed that passed through the experimental system. There was a linear dependence between the particle recovery in the underflow and the water flux, with a slope dependent on the relative selectivity between the different particle species. The analysis was examined using a water flux of up to $0.0167 \mathrm{~m}^{3} /\left(\mathrm{m}^{2} \mathrm{~s}\right)$. The results agreed well with the analytical and DEM models within the aforementioned water flux range. 
With the second approach, electrostatic formation of liquid marbles was investigated as an alternative mechanism for collecting hydrophilic glass ballotini particles into a water drop. In these experiments curious electrostatic phenomena were observed, resulting in the formation of particle agglomerates. Fine hydrophilic and hydrophobic particles on a charged substrate were seen to intermittently jump across a gap of several millimetres to penetrate the surface of a hanging water drop. The substrate was wrapped in Teflon (PTFE) tape, which provided an ideal substrate for frictional charging. Within around 300 milliseconds, the drop filled up like a 'sack of marbles'.

Charge and volume data analysis of these experiments revealed that there is a tendency of the particles to jump to the drop intermittently in sudden 'cascades' or 'avalanches', rather than through gradual and incremental transfer as the substrate was moved toward the hanging drop. Although the distance between the substrate and the drop was important in affecting particle lift, the substrate speed had relatively little effect on the onset of the particle 'avalanche'. This interesting phenomenon was also observed using different organic liquids such as glycerine and hexane.

Overall, it was apparent that a limited selectivity between hydrophilic and hydrophobic particles can be achieved through direct interaction between a gaseous dispersion of the particles and a system of water drops. However, the level of selectivity was deemed to be insufficient for justifying the development of a successful industrial separation. The data sets generated from this work can be useful in any follow-up work in improving our understanding of the precise particle-drop interception, and final interaction. Electrostatic forces were clearly powerful across significant distances, and offered the potential for developing innovative methods for processing fine, dry particles. This approach may also prove useful in the formation of both hydrophilic and hydrophobic liquid marbles. 


\section{PUBLICATIONS}

Liyannarachchi, K.R., Webber, G.B., van Netten, K., Moreno-Atanasio, R. and Galvin, K.P., (2014), Selective Collection of Fine Particles by Water Drops, Journal of Advanced Powder Technology, Vol.25, Issue 4, p.1311-1318.

Liyanaarachchi, K.R., Ireland, P.M., Webber, G.B. and Galvin, K.P. (2013), Electrostatic Formation of Liquid Marbles and Agglomerates, Journal of Applied Physics Letters, Vol.103, Issue 5, p.054105 - 054104.

Liyanaarachchi, K., Ireland P., Webber, G. and Galvin, K. (2013), Electrostatic formation of liquid marbles, Proceedings ESA Annual Meeting on Electrostatics 2013, Florida, United States.

Liyanaarachchi, K.R., Galvin, K.P. and Webber G.B. (2012), Selective Collection of Fine Particles by Water Drops, 12th AIChe Proceedings, Pennsylvania, United States. Paper ID: 266477.

Liyanaarachchi, K.R., Webber G.B. and Galvin, K.P. (2012), Selective Collection of Fine Particles by Water Drops, Proc. $28^{\text {th }}$ Australian Colloid \& Surface Science Student Conference, Newcastle, Australia.

Galvin, K.P., Webber, G., Mason, M. and Liyanaarachchi, K.R. (2010), Inverse Flotation - A New Method of Fine Particle Beneficiation, Proceedings of CHEMECA 2010, Adelaide, Australia.

\section{REPORTS}

Galvin, K.P., Liyanaarachchi K.R., Iveson, S. and Webber G.B. (2012), A Step Change in Fine Coal Beneficiation - Inverse Flotation, ACARP Report 2012, Australia. 


\section{TABLE OF CONTENTS}

List of Figures $\quad$ XV

List of Tables $\quad$ xxi

Nomenclature $\quad$ xxii

CHAPTER 1

INTRODUCTION

$\begin{array}{ll}\text { 1.1 Thesis Overview } & 02\end{array}$

1.2 First Approach - Collecting Particles in an Air Stream 03

1.3 Second Approach - Electrostatic Transport of Particles 06

$\begin{array}{ll}1.4 \text { Aims and Objectives } & 07\end{array}$

$\begin{array}{ll}\text { 1.5 Thesis Outline } & 08\end{array}$

\section{CHAPTER 2}

FORCES AND PHENOMENA INVOLVED IN PARTICLE-LIQUID-GAS INTERACTIONS

2.1 Introduction 11

$\begin{array}{ll}\text { 2.2 Surface Forces } & 11\end{array}$

$\begin{array}{ll}2.2 .1 & \text { van der Waals Interactions }\end{array}$

2.2.2 Electrostatics Forces in Air 14

2.2.3 Classical DLVO Theory of Surface Forces 15

$\begin{array}{lll}\text { 2.2.4 Extended DLVO Theory } & 17\end{array}$

$\begin{array}{ll}\text { 2.2.4.1 Non DLVO Forces } & 17\end{array}$

$\begin{array}{ll}\text { 2.2.4.2 Hydrophobic Phenomena } & 18\end{array}$

2.3 Wetting and The Concept of Contact Angle 19

$\begin{array}{ll}\text { 2.3.1 Wetting } & 19\end{array}$

2.3.2 The Concept of Contact Angle 20 
2.3.4 Wetting and Adhesion 23

2.3.4.1 The Work of Cohesion $\left(W_{\mathrm{c}}\right) \quad 23$

2.3.4.2 The Work of Adhesion $\left(W_{\mathrm{A}}\right) \quad 24$

2.3.5 Young-Laplace Equation 25

$\begin{array}{lr}\text { 2.4 Surface Energy and Spreading } & 28\end{array}$

$\begin{array}{lll}2.4 .1 & \text { Surface Energy } & 28\end{array}$

$\begin{array}{lll}2.4 .2 & \text { Spreading } & 29\end{array}$

2.4.3 Spreading Co-efficient $(S) \quad 29$

2.5 Dynamic Contact Angles and Kinetics of Wetting 30

2.5.1 Contact Angle Hysteresis 30

2.5.2 Dynamic Contact Angles 31

2.5.3 Kinetics of Wetting 32

2.5.3.1 Hydrodynamic Model of Kinetics of Wetting 33

2.5.2.2 Molecular Kinetics Theory of Wetting 35

2.5.2.3 Combined (Microhydrodynamic) Model 36

$\begin{array}{ll}\text { 2.6 Electrostatics } & 37\end{array}$

$\begin{array}{lll}\text { 2.6.1 Introduction to Electrostatics } & 37\end{array}$

2.6.2 Conductors and Non Conductors (Dielectric Particles/Insulators) 38

2.6.3 Mechanisms of Particle Charging 38

2.6.3.1 Induction 39

2.6.3.2 Conduction $\quad 39$

2.6.3.3 Tribocharging (Triboelectrification /Friction Charging) 40

2.6.4 Effects of Physical Conditions 41

$\begin{array}{ll}\text { 2.7 Summary } & 42\end{array}$ 


\section{CHAPTER 3}

APPLICATIONS INVOLVING PARTICLE-LIQUID-GAS INTERACTIONS

3.1 Introduction 45

$\begin{array}{ll}\text { 3.2 Hydrodynamics } & 45\end{array}$

3.2.1 Forces Acting on Particles in Fluids 45

3.2.1.1 Gravitational Force $\left(F_{G}\right) \quad 46$

3.2.1.2 Buoyancy Force $\left(F_{B}\right) \quad 46$

3.2.1.3 Drag Force $\left(F_{D}\right) \quad 47$

3.2.2 Flow (Particle Drag) Regimes 48

3.2.3 Terminal Settling Velocity $\left(v_{s}\right) \quad 49$

3.2.4 Flow Fields 51

3.3 Particle Interactions through Liquid 52

3.3.1 Flotation Probabilities $\quad 52$

3.3.2 Bubble -Particle Collision 53

3.3.2.1 Probability of Collision and Bubble-Particle Collision Models 55

3.3.3 Bubble-Particle Attachment 59

3.3.3.1 Probability of Attachment or Adhesion $\left(P_{a}\right) \quad 63$

3.3.4 Bubble-Particle Detachment 64

3.3.4.1 Probability of Detachment $\left(P_{d}\right) \quad 65$

3.3.5 Flotation Kinetics 66

3.3.5.1 Flotation Kinetics and Operating Variables 66

$\begin{array}{lll}\text { 3.3.6 Fine Particle Flotation } & 70\end{array}$

3.4 Particle Interactions through Air 71

3.4.1 Wet Scrubbers 71

$\begin{array}{ll}\text { 3.4.2 Granular Filtration } & 74\end{array}$

3.4.3 Recent Research on Fine Particle Separation 75 
3.4.5 Electrostatic Applications

(a) Electrostatic Precipitation $\quad 79$

$\begin{array}{ll}\text { (b) Electrostatic Coatings } & 79\end{array}$

(c) Electrostatic Fluidised Beds $\quad 80$

(d) Electrostatic Separators $\quad 81$

$\begin{array}{lr}\text { 3.5 Summary } & 84\end{array}$

CHAPTER 4

MODEL DEVELOPMENT

$\begin{array}{ll}4.1 \text { Introduction } & 87\end{array}$

$\begin{array}{ll}\text { 4.2 A Model for Drop-Particle Collision } & 87\end{array}$

4.2.1 Drop-Particle Collision Model for $u_{d} \gg u_{p}$

4.2.2 Drop-Particle Collision Model for $u_{p} \gg u_{d}$

$\begin{array}{ll}\text { 4.3 Discrete Element Model } & 95\end{array}$

$\begin{array}{ll}\text { 4.4 Discussion } & 96\end{array}$

CHAPTER 5

EXPERIMENTAL METHOD FOR SELECTIVE COLLECTION OF FINE PARTICLES

$\begin{array}{ll}5.1 \text { Introduction } & 100\end{array}$

$\begin{array}{ll}\text { 5.2 Preliminary Single Drop Experiments } & 100\end{array}$

5.3 Development of Multiple Drop Experimental System 102

5.3.1 Feeding Configuration 105

$\begin{array}{ll}\text { 5.3.2 Collision Chamber and the Vacuum Pump } & 106\end{array}$

5.3.3 Water Distribution Chamber Design and Cyclone 106 
5.4.1 Methodology and Materials for Single Drop Experiments

\section{CHAPTER 6}

\section{RESULTS AND DISCUSSION}

6.2 Results of the Preliminary Single Drop Experiments

6.3 Results of the Multiple Drop Experimental System

6.3.1 Recovery Variation with the Water Flux

6.3.2 Comparison of Experimental, Analytical and DEM Results

6.3.3 Effect of Surface Modification

6.3.4 Recovery Variation with Different Particle Sizes

6.3.5 Effect of Particle Size and Shape

6.3.6 Effect of Different Size Hydrophobic Particles

6.3.7 Effect of Different Particle Types

6.3.8 Effect of Air Flow Rates

6.3.9 Recovery Variation with the Water Quality Changes

\section{CHAPTER 7}

\section{ELECTROSTATIC EXPERIMENTS}


7.4.1 Identifying the Experimental Limits and the Phenomena

7.4.2 Hypothesis

7.4.2.1 Positive Feedback Mechanisms

7.4.2.2 Negative Feedback Mechanisms

7.4.3 Charge Measurements and Volume Changes with Time

7.4.4 Charge Measurements and Variable Speeds

7.4.5 Hydrophilic Particle Behaviour with Different Liquid Drops

\section{CHAPTER 8}

\section{CONCLUSIONS AND RECOMMENDATIONS}

8.1 Conclusions 172

$\begin{array}{ll}\text { 8.2 Recommendations } & 174\end{array}$

$\begin{array}{ll}\text { REFERENCES } & 175\end{array}$

$\begin{array}{ll}\text { APPENDICES } & 188\end{array}$ 


\section{LIST OF FIGURES}

\section{CHAPTER 1}

Figure 1.1: Comparison between (a) conventional flotation where particles in water interacting with rising air bubbles and (b) the first approach investigated in this research where falling drops interact with particles suspended in air.

Figure 1.2: Schematic representation of the engulfment of fine silica particles into a water drop through the air-water interface.

Figure 1.3: Schematic of transfer of electrostatically charged glass ballotini to a hanging pendent water drop.

\section{CHAPTER 2}

Figure 2.1: Origin of surface tension forces acting at a liquid surface.

Figure 2.2: van der Waals interactions between two dipole molecules.

Figure 2.3: Interaction energy between a particle and a bubble as per the DLVO Theory

Figure 2.4: Contact angles for (a) Partial wetting (b) non-wetting.

Figure 2.5: Equilibrium contact between a liquid drop and a solid in a gas.

Figure 2.6: Droplet spreading on a solid surface.

Figure 2.7: Diagram of cohesion process.

Figure 2.8: Diagram of adhesion process.

Figure 2.9: Work of adhesion between a particle and an air bubble in conventional flotation.

Figure 2.10: Surface curvature and pressure.

Figure 2.11: Advancing $\left(\theta_{a}\right)$ and receding $\left(\theta_{r}\right)$ contact angles.

Figure 2.12: Dynamic contact angle $\left(\theta_{D}\right)$ variation with respect to contact line velocity $\left(v_{e}\right)$.

Figure 2.13: Spreading of microscopic precursor film in front of macroscopic 
edge (Butt et al., 2003).

Figure 2.14: Parameters of moving contact line (Bertrand et al., 2009).

Figure 2.15: Charging by Induction (1) Charged rod brought close to an insulated conductor (2) Charge Separation (3) Earth Conductor (4) Remove charged rod.

Figure 2.16: Insulated object charging by conduction.

Figure 2.17: Charging by Friction (a) Before rubbing (b) After rubbing.

\section{CHAPTER 3}

Figure 3.1: Forces acting on a downward moving particle in a fluid.

Figure 3.2: Drag Co-efficient as a function of Reynolds number for different shapes (Source: Haider and Levenspiel, 1989).

Figure 3.3: Streamlines of a laminar flow and a turbulent flow.

Figure 3.4: Three zones around the particle in flotation process: Zone 1 . Hydrodynamic; Zone 2. Diffusiopheretic Zone 3. Surface Force Zone (Ralston et al., 1998).

Figure 3.5: Velocity components ( $V_{r}$, radial velocity and $V_{\alpha}$, tangential velocity) of the particle approaching a bubble travelling with an upward velocity of $V_{b}$.

Figure 3.6: Trajectories of particles on streamlines.

Figure 3.7: Stages of forming bubble-particle aggregate: (a) Film thinning (b) Film rupture.

Figure 3.8: Collision angle and the colliding area of the bubble-particle collision. $\quad 61$

Figure 3.9: Particle attached to a larger air bubble (Nguyen et al., 2003).

Figure 3.10: Recovery of different size of sulfide minerals after 60 seconds flotation batch tests (Source :Jowett, 1980).

Figure 3.11: Flotation rate constant variation with particle size for batch flotation of several sulphide minerals. (Source: Ahmed \& Jameson, 1989).

Figure 3.12: Inertial impaction of particles on a water drop.

Figure 3.13: Interception impaction of particles on a water drop.

Figure 3.14: Inverse Flotation via overflowing trough. 
Figure 3.15: Microscope Images of water drops (a) Silica particles inside test drop (no coal in feed). (b) No coal particles found inside the test drop, though some coal evident at drop surface. (c) Silica particles inside drop (silica and coal feed).

Figure 3.16: Self coating particles in Liquid Marble (Source: McHale and Newton, 2011)

$\begin{array}{lr}\text { Figure 3.17: Electrostatic Coating. } & 80\end{array}$

Figure 3.18: Simple electrostatic fluidised-bed coating system. 80

Figure 3.19: High tension electrostatic separator. 82

Figure 3.20: Separation process of a triboelectric separator. 83

\section{CHAPTER 4}

Figure 4.1: Schematic representation of the theoretical collision zone showing the dimensions, and the direction of flux of the particles (horizontal) and water drops (downward).

Figure 4.2: Schematic representation of the volume swept by a falling drop, that may be approximated by a cylinder with diameter equal to the drop, $D_{d}$, and height of $d Z$, the distance the drop falls in time $d t$.

Figure 4.3: Estimated particle collision recoveries obtained using the water flux values given in Table 4.1. The two different models were used with $X=0.00234 \mathrm{~m}$, $D_{d}=0.003 \mathrm{~m}, u_{p}=6 \mathrm{~m} / \mathrm{s}$, and $u_{d}=1.04 \mathrm{~m} / \mathrm{s}$.

Figure 4.4: Three dimensional graph showing the recovery variation with the water flux and the drop diameter.

Figure 4.5: Simulated and estimated recovery as a function of the water flux. It is evident that the recovery values converge towards the idealised model when the particle velocity greatly exceeds the drop velocity.

\section{CHAPTER 5}

Figure 5.1: Single drop experimental set up, incorporating a vibratory support for energizing the fine particles located below the hanging drop.

Figure 5.2: Schematic of the multi-drop experimental system.

Figure 5.3: Initial proto type of the laboratory scale experimental system. 
Figure 5.4: Air inlet manifold around the feeding column.

Figure 5.5: Design of water distribution chamber.

Figure 5.6: Modified collision chamber with water distributing capillary tubes.

Figure 5.7: Flow straightner visible at the rear of the image through which the air entered.

Figure 5.8: Schematic diagram of final laboratory scale experimental system.

Figure 5.9: 3D Model of the Experimental System.

Figure 5.10: Photograph of the actual laboratory scale experimental system

(1)Feeding column (2) Water distribution chamber (3) Collision chamber

(4) Cyclone (5) Tube connecting to vacuum pump (6) Collecting tanks

Figure 5.11:Velocity profiles of the airflow along the centre line of the collision chamber measured by Velocicalc ${ }^{\circledR}$ and using an air rotameter.

Figure 5.12: Measurement points for horizontal air velocity profiles.

Figure 5.13: Horizontal airflow velocity variation at different heights within the collision chamber, measured for three horizontally distributed planes.

Figure 5.14:Velocity profile of the particle-air plume inside the collision chamber. 118

\section{CHAPTER 6}

Figure 6.1: Photographs of pendent water drops after exposure to (a) glass ballotini and (b) coal particles. In both cases, pendent drops of water were saturated by the particles used.

Figure 6.2: Microscopic image of water drop showing spherical glass ballotini particles.

Figure 6.3: Photographs of pendent water drops containing glass ballotini and coal particles (a) after vibrating the mix under the drop, (b) after sprinkling from above. 123

Figure 6.4: Comparison between the predicted recovery and the actual recovery. Blue line corresponds to underflow recovery of as received glass ballotini $(23-47 \mu \mathrm{m})$ versus wash water flux. The straight line represents the best fit for the experimental data.

Figure 6.5:The experimental underflow recovery and the estimated recoveries shown as a function of the wash water flux. 
Figure 6.6: Recovery of cleaned glass ballotini and "as received" glass ballotini in the size range $23-47 \mu \mathrm{m}$.

Figure 6.7: Underflow recovery of cleaned glass ballotini in 17-36 $\mu \mathrm{m}$ and 23-47 $\mu \mathrm{m}$ size ranges.

Figure 6.8: Underflow recovery of different size range clean silica; $8-29 \mu \mathrm{m}$, $16-42 \mu \mathrm{m}$, and $21-54 \mu \mathrm{m}$. For comparison, the recovery of $23-47 \mu \mathrm{m}$ cleaned ballotini is also shown.

Figure 6.9: Microscope Images of (a) glass ballotini (b) silica and (c) coal in 38-45 microns size range.

Figure 6.10: Underflow recovery of coal covering different size ranges; 30-78 $\mu \mathrm{m}, 29-105 \mu \mathrm{m}$ and 24-116 $\mu \mathrm{m}$.

Figure 6.11: Recovery of cleaned glass ballotini at two different airflow rates; $1.4 \mathrm{~m} / \mathrm{s}$ and $1.75 \mathrm{~m} / \mathrm{s}$.

Figure 6.12: Underflow recoveries for $21-54 \mu \mathrm{m}$ cleaned silica with reverse osmosis (RO) water and tap water.

Figure 6.13: Recovery of 21- $54 \mu \mathrm{m}$ cleaned silica variation with the increasing salt (Aluminium sulphate) concentration. Salt concentration changed from $50 \mathrm{mg} / \mathrm{L}$ up to $2000 \mathrm{mg} / \mathrm{L}$. Conductivity of water changed from $247 \mu \mathrm{S}$ to $966 \mu \mathrm{S}$. All these recoveries were obtained at a constant water flux of $0.0143 \mathrm{~m}^{3} / \mathrm{m}^{2} \mathrm{~s}$.

Figure 6.14: Recovery of 24-116 $\mu \mathrm{m}$ coal and 21-54 $\mu \mathrm{m}$ silica in the underflow for $50 \% \mathrm{w} / \mathrm{w}$ silica-coal feed mixture.

Figure 6.15: Blocking mechanism used to explain the higher selectivity obtained for the mixture of the 24-116 $\mu \mathrm{m}$ coal and 21-54 $\mu \mathrm{m}$ silica.

\section{CHAPTER 7}

Figure 7.1: Experimental set up.

Figure 7.2: Image showing the initial distance of $40 \mathrm{~mm}$ between the drop and the Teflon wrapped substrate.

Figure 7.3: Image showing particles of spherical ballotini being transfer to a hanging water drop across an air gap. (Image captured with the OCA 20 tensiometer camera)

Figure 7.4: The high speed video footage produced high resolution images during the particle transfers.

Figure 7.5: As received ballotini with the hanging water drop. 
Figure 7.7: Drop height variation with the time(s). Particle bed lifting started after $26 \mathrm{~s}$.

Figure 7.8: Drop deformations associated with the manual movement of the susbtrate in the absence of particles.

Figure 7.9: Image schematic of the proposed bed cohesion mechanism. The electric field lines converge on the drop, putting the particle bed immediately under the drop under a radial compressive stress in the plane of the bed surface.

Figure 7.10: Cumulative change in the charge transferred of the drop without particles on the bed.

Figure 7.11: Cumulative changes in the drop volume (solid curve), corresponding to cumulative changes in the charge transfer to the drop (broken curve). Subsequent to initial particle transfer, the volume and charge changes correspond closely.

Lifting of substrate started after 28 seconds.

Figure 7.12: Charge transferred to the drop when the stage is advanced at a steady velocity $(200 \mu \mathrm{m} / \mathrm{s})$ then halted when particle transfer is observed. The solid curve indicates measured charge, and the broken curve indicates induction of charge on the drop. An avalanche occurred in the first case, but not in the second.

Figure 7.13: Charge measurements corresponding to different substrate velocities against the time.

Figure 7.14: Charge measurements corresponding to different substrate velocities against the approach displacements.

Figure 7.15: Image sequence of interaction between a bed of glass ballotini and a hanging drop of glycerine.

Figure 7.16: Image sequence of interaction between a bed of glass ballotini and a hanging drop of hydrophobic hexane.

Figure 7.17: Image sequence of interaction between a bed of PVC particles and a hanging water drop. Bed was driven manually.

Figure 7.18: (a) Coal 75-90 $\mu \mathrm{m}$ particles show a clear branching and less deformation compared to (b) coal $38-45 \mu \mathrm{m}$ particles.

Figure 7.19: Image sequence of interaction between a bed of coal $(90 \% \mathrm{w} / \mathrm{w})$ and silica $(10 \% \mathrm{w} / \mathrm{w})$ particles in $75-90$ micron size range with a hanging water drop. 


\section{LIST OF TABLES}

Table 3.1: Values for the Richardson-Zaki Index (n)

Table 4.1: Calculation of the water flux (W) based on experimental values of the water flow rate. These values of $\mathrm{W}$ were used to estimate the recovery values shown in Figure 4.3.

Table 5.1: Malvern Mastersizer 2000 size measurements of the different material types used in the multiple drop experiments.

Table 7.1: Properties of different particles used in electrostatic experiments and their sources.

Table 7.2: Properties of different liquids used in electrostatic experiments and their sources. 


\section{NOMENCLATURE}

$a$

$b$

$d_{b}$

$d_{p}$

$d_{50}$

$d t$

$d Z, d z$

$g$

$k_{B}$

$m$

$\mathrm{m}^{\prime}$

$m_{f}$

$n$

n'

$n_{d}$

$\Delta p$

$p$

$q$

$q_{1}, q_{2}$

$r_{b}$

$r_{p}$

$s$

$t$

$t_{\text {at }}$ or $t_{\text {ind }}$

$t_{c}$

$t_{\text {contact }}$ or $t_{s}$

$u_{d}$

$u_{p}$

V

$v_{p}$

$v_{t}$

$V_{b}$

Ve

$X$

$x, y$

$V_{r}$

$V_{\phi}$
Attraction constant for molecules $\left(\mathrm{Pa} \mathrm{m}^{6}\right.$ $/ \mathrm{mol}^{2}$ )

Actual volume of the molecules $\left(\mathrm{m}^{3} / \mathrm{mol}\right)$

Bubble diameter (m)

Particle diameter $(\mathrm{m})$

Median particle diameter (m)

Small time period (s)

Small distance $(\mathrm{m})$

Gravitational acceleration $\left(\mathrm{m} / \mathrm{s}^{2}\right)$

Boltzmann constant $(\mathrm{J} / \mathrm{K})$

Mass of the particle $(\mathrm{kg})$

Number of drops per row

Mass of the fluid displaced by the particle

$(\mathrm{kg})$

Number of moles

Order of flotation

Number of drops in $1 \mathrm{~m}^{3}$

Capillary pressure difference between two phases, Laplace pressure $(\mathrm{Pa})$

External pressure $(\mathrm{Pa})$

Charge (C)

Small charges $(\mathrm{C})$

Radius of the bubble (m)

Radius of the particle $(\mathrm{m})$

Falling height (m)

Time (s)

Bubble-particle attachment time (s)

Collision time (s)

Contact time (s)

Vertically falling velocity of the water drops $(\mathrm{m} / \mathrm{s})$

Horizontal velocity of the particles across the collision chamber $(\mathrm{m} / \mathrm{s})$

Relative velocity of the particle with respect to the water drop

Particle velocity relative to the fluid $(\mathrm{m} / \mathrm{s})$

Hindered Settling Velocity $(\mathrm{m} / \mathrm{s})$

Upward bubble velocity $(\mathrm{m} / \mathrm{s})$

Velocity $(\mathrm{m} / \mathrm{s})$

Smaller Length (m)

Distances (m)

Radial velocity $(\mathrm{m} / \mathrm{s})$

Tangential velocity $(\mathrm{m} / \mathrm{s})$ 
$\triangle A$

$A_{d}$

$C_{D}$

$C_{p}$

$\mathrm{Cu}$

$\mathrm{Ca}$

D

$D_{d}$

E

$E_{k}$

$E^{\prime}$

$E_{1}$

F

$F_{B}$

$F_{C}$

$F_{C}^{\prime}$

$F_{D}$

$F_{G}$

$F_{g}$

$F_{h}$

$F_{p}$

$F_{t}$

$F^{\prime}$

G

$G_{c}$

$G_{f}$

G'

$\Delta \mathrm{G}^{\prime}, \Delta \mathrm{G}_{s g}^{\prime}, \Delta \mathrm{G}_{s l}^{\prime}$

$H^{\prime}$

H

$\mathrm{K}^{0}$

K

$L$

$L_{m}$

$N$

$\mathrm{N}_{\mathrm{R}}$

$N^{\prime}$

$P$

$P_{a}$

$P_{a-D F}$

Surface area, reference cross sectional area $\left(\mathrm{m}^{2}\right)$

Change in surface area $\left(\mathrm{m}^{2}\right)$

Area of a spherical water drop $\left(\mathrm{m}^{2}\right)$

Drag coefficient

Concentration of floatable particle $\left(\mathrm{kg} / \mathrm{m}^{3}\right)$

Cunningham correction factor

Capillary number

Center- center distance between particles

(m)

Diameter of the water drop (m)

Electric field intensity or strength (N/C)

Kinetic energy of the particles $(\mathrm{J})$

Energy Barrier (J)

Dispersion force $(\mathrm{N})$

Force $(\mathrm{N})$

Buoyancy force $(\mathrm{N})$

Centrifugal force $(\mathrm{N})$

Capillary force $(\mathrm{N})$

Hydrodynamic drag force $(\mathrm{N})$

Gravitational force $(\mathrm{N})$

Particle weight $(\mathrm{kg})$

Hydrostatic Pressure Force (N)

Hydrodynamic pressing force $(\mathrm{N})$

Inertial forces $(\mathrm{N})$

Helmholtz free energy $(\mathrm{J})$

Particle volume flux $\left(\mathrm{m}^{3} / \mathrm{s}\right)$

Volume flux of particles that collide with drops $\left(\mathrm{m}^{3} / \mathrm{s}\right)$

Feed volume flux of particles $\left(\mathrm{m}^{3} / \mathrm{s}\right)$

Gibbs free energy $(\mathrm{J})$

Free energy change $(\mathrm{J})$

Contact angle hysteresis

Vertical distance $(\mathrm{m})$

Equilibrium frequency of the random

molecular displacements $(\mathrm{Hz})$

Stokes Number

Macroscopic length scale (m)

Microscopic length scale (m)

Number of drops

Rows of drops

Number

Overall Probability or capture efficiency

of particles

Probability of adhesion or attachment

efficiency

Probability of attachment as Dobby and

Finch model 


\begin{tabular}{|c|c|}
\hline$P_{a-Y}$ & $\begin{array}{l}\text { Probability of particle-bubble attachment } \\
\text { as per Yoon model }\end{array}$ \\
\hline$P_{a-Y L}$ & $\begin{array}{l}\text { Probability of attachment as per Yoon- } \\
\text { Mao model }\end{array}$ \\
\hline$P_{c}$ & Probability of collision or collision efficiency \\
\hline$P_{c-F R}$ & $\begin{array}{l}\text { Collision efficiency as per Flint and } \\
\text { Howarth \& Reay and Ratcliff }\end{array}$ \\
\hline$P_{c-G S E}$ & $\begin{array}{l}\text { Collision efficiency as per Generalised } \\
\text { Sutherland Model }\end{array}$ \\
\hline$P_{c-L B}$ & $\begin{array}{l}\text { Collision efficiency as per Langmuir and } \\
\text { Blodgett model }\end{array}$ \\
\hline$P_{c-S U}$ & $\begin{array}{l}\text { Collision efficiency as per Sutherland } \\
\text { model }\end{array}$ \\
\hline$P_{c-Y L}$ & $\begin{array}{l}\text { Collision efficiency as per Yoon and } \\
\text { Luttrell model }\end{array}$ \\
\hline$P_{d}$ & Probability of detachment \\
\hline$P_{s}$ & $\begin{array}{l}\text { Probability of forming a particle-bubble } \\
\text { aggregate }\end{array}$ \\
\hline$Q$ & $\begin{array}{l}\text { Volume of space associated with the first } \\
\text { row of drops }\left(\mathrm{m}^{3}\right)\end{array}$ \\
\hline$R$ & Recovery \\
\hline$R^{\prime}$ & Ideal gas constant $(\mathrm{J} / \mathrm{K}$ mol $)$ \\
\hline$R, \check{R}, R_{1}, R_{2}, r$ & Radius of the liquid curvature (m) \\
\hline$R_{c}$ & $\begin{array}{l}\text { Stream of collision radius (grazing radius) } \\
(\mathrm{m})\end{array}$ \\
\hline$R(t)$ & Spreading Rate $(\mathrm{m} / \mathrm{s})$ \\
\hline$R_{a v}$ & Average radius curvature $(\mathrm{m})$ \\
\hline$R e_{p}$ & Particle Reynolds number \\
\hline $\operatorname{Re}_{b}$ & Reynolds number of the bubble \\
\hline$S$ & Spreading coefficient \\
\hline$S^{\prime}$ & Entropy of the system $(\mathrm{J} / \mathrm{K})$ \\
\hline$S_{L / S}$ & $\begin{array}{l}\text { Spreading coefficient of a liquid over a } \\
\text { solid }\end{array}$ \\
\hline$T$ & Absolute temperature $(\mathrm{K})$ \\
\hline$U$ & Internal energy of the system $(\mathrm{J})$ \\
\hline$V$ & Volume or molar volume $\left(\mathrm{m}^{3}\right)$ \\
\hline$V_{d}$ & Volume of the spherical drop $\left(\mathrm{m}^{3}\right)$ \\
\hline$V_{p}$ & Volume of the particle $\left(\mathrm{m}^{3}\right)$ \\
\hline$W$ & $\begin{array}{l}\text { Water volume flux across the collision } \\
\text { chamber }\left(\mathrm{m}^{3} / \mathrm{s}\right)\end{array}$ \\
\hline$W_{A}$ & Work of adhesion $(\mathrm{J})$ \\
\hline$W_{\mathrm{c}}$ & Work of cohesion $(\mathrm{J})$ \\
\hline$W_{s}$ & Work of spreading $(\mathrm{J})$ \\
\hline$X$ & Distance along the collision zone (m) \\
\hline$Y$ & Distance across the collision chamber $(\mathrm{m})$ \\
\hline$Z$ & $\begin{array}{l}\text { Height of the zone parallel with the falling } \\
\text { drops }(\mathrm{m})\end{array}$ \\
\hline
\end{tabular}




\section{Greek Letters}

$\alpha$

$\gamma, \gamma_{\mathrm{A}}, \gamma_{\mathrm{B}}, \gamma_{\mathrm{AB}}$

$\gamma_{l g}$

$\gamma_{s g}$

$\gamma_{s l}$

$\varepsilon$

$\varepsilon_{0}$

$\varepsilon_{r}$

$\varepsilon_{1}$

$\delta$

$\zeta$

$\eta$

$\theta$

$\theta_{a}$

$\theta_{D}$

$\theta_{e}$

$\theta_{m}$

$\theta_{r}$

$\theta_{S}$

$\theta_{t}$

$\lambda$

$\mu$

$\pi_{d}$

$\pi_{e}$

$\pi_{s}$

$\mathrm{p}$

$\rho_{f}$

$\rho_{p}$

$\sigma^{\prime}$

$\sigma_{1}, \sigma_{2}$

$\Phi$

$\phi_{d}$
Fraction of the two dimensional projected area

Interfacial tensions (or surface energies) of the relevant interfaces $(\mathrm{N} / \mathrm{m})$

Interfacial tension between the liquid and gas $(\mathrm{N} / \mathrm{m})$

Interfacial tension between the solid and gas $(\mathrm{N} / \mathrm{m})$

Interfacial tension between the solid and gas $(\mathrm{N} / \mathrm{m})$

Permittivity (F/m)

Permittivity of vacuum (F/m)

Relative permittivity or relative dielectric constant

Void fraction

Diameter of the flow cylinder (m)

Friction co-efficient

Collection efficiency of particles by interception

Contact angle

Advancing contact angle

Dynamic contact angle

Equilibrium contact angles

Microscopic angle

Receding contact angle

Static contact angle

Angle of tangency

Average distance of each displacement

(m)

Dynamic viscosity of liquid (Pa s)

van der Waals force disjoining pressure

(Pa)

Electrostatic disjoining pressure $(\mathrm{Pa})$

Structural disjoining pressure $(\mathrm{Pa})$

Disjoining pressure $(\mathrm{Pa})$

Fluid density $\left(\mathrm{kg} / \mathrm{m}^{3}\right)$

Density of the particle $\left(\mathrm{kg} / \mathrm{m}^{3}\right)$

Impaction parameter for a potential (ideal)

flow

Surface charge densities of the two

particles (C)

Ratio between of the surface area of a particle having the same volume as a sphere to the surface area of the particle Volume fraction of the drops 
Volume fraction of particles

Collision angle

Maximum Collision angle 


\section{Chapter 1}

\section{INTRODUCTION}

1.1 Thesis Overview

1.2 First Approach - Collecting Particles in an Air Stream

1.3 Second Approach - Electrostatic Transport of Particles

1.4 Aims and Objectives

1.5 Thesis Outline 


\subsection{Thesis Overview}

This Thesis is concerned with the development of novel methods for promoting the motion of fine particles in order to achieve collision with drops of water. This interaction is promoted routinely in wet scrubbers for the purpose of filtering virtually all particulate matter from the gaseous stream. Thus the usual approach does not provide a uniform and well defined set of conditions for assessing the potential selectivity of different types of particles in the interaction. This study was thus focussed on promoting well defined conditions for observing and measuring the capture of different particles. The potential for extending the work to develop new separation technologies was also a key motivation of the work.

There were two approaches adopted to investigate the interactions of fine particles with drops of water. The usual approach involves the application of a carrier fluid such as air to entrain and deliver the particles to the target drops. This first approach formed a major part of the present study. A second approach, which involved no airflow was also investigated, based on the application of the electrostatic force. This force was used to carefully carry the particles to the target drop. Thus, there were two key parts to the work presented in this Thesis, both offering the potential for developing, in principle, a new technology for separating particles according to differences in their surface properties.

This work was motivated by interests in achieving firstly a controlled state of collision between particles and drops of water and ideally achieving a separation between the particles collected by the drops and those particles that fail to be collected. Of more specific interest was the degree of selectivity between hydrophilic and hydrophobic particles. Hydrophobic particles should be more likely to either detach or deflect, especially when the angle of interception is large. Hydrophilic particles, however, will tend to become fully engulfed by the water drop and sediment to the base of the drop.

This work was motivated by interests in achieving firstly a controlled state of collision between particles and drops of water and ideally achieving a separation between the particles collected by the drops and those particles that fail to be collected. There are significant benefits in operating within the dry state, and in generating a dry product. 
These benefits are apparent in coal and mineral processing where lower moisture is required. The surrounding air phase of the water drop, which has low viscosity of order $10^{-5} \mathrm{~Pa}$.s, offers relatively little resistance, hence the effectiveness of collisions tends to be very high compared to the particle-air bubble collision which occurs in a slurry medium. Upon collision, a process of dynamic wetting, followed by adhesion, or complete engulfment ensues. Of more specific interest was the degree of selectivity between hydrophilic and hydrophobic particles. Hydrophobic particles should be more likely to either detach or deflect, especially when the angle of interception is large. Hydrophilic particles, however, will tend to become fully engulfed by the water drop and sediment to the base of the drop.

\subsection{First Approach - Collecting Particles in an Air Stream}

The need to introduce an air flow presented a range of challenges, and hence the need to develop and optimize a suitable system. Fluidized bed systems are one of the most effective approaches for energizing and controlling fine particles, involving the suspension of the particles through the application of air flow up through the bed. The fine particles elutriate into the zone above, disperse to the wall, and then return to the bed. The solids concentration above the bed is exceedingly low, however, and the air flow must be sufficient to fluidize the bed and achieve elutriation. These facts place significant constraints on the application of fluidization to this problem. Further, the management of the water drops within the system is very difficult, given that a concentrated bed of solids exists below, and hence there is no obvious way to disengage the water drops from such a system. Thus the airflow needs to be directed horizontally into a collision chamber allowing the water drops to collide with the particles, settle, and disengage from the flow.

The first approach can be thought of as, in effect, the "inverse" of flotation. In conventional flotation, air bubbles in the mineral suspension carry the hydrophobic particles upwards towards the froth, leaving the hydrophilic particles in the suspension as shown in Figure 1.1(a). In the present study, the rising gas bubbles are replaced by falling liquid drops, and the water-based suspension is replaced by a gas dispersion. In turn, the hydrophilic particles are transported downward by the falling water drops, leaving the hydrophobic particles in the gas stream as shown in Figure 1.1(b). 


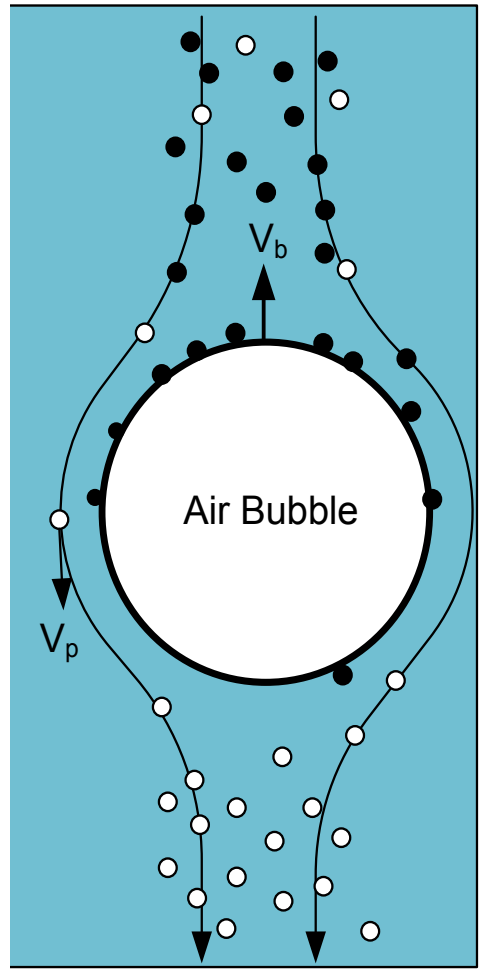

(a)

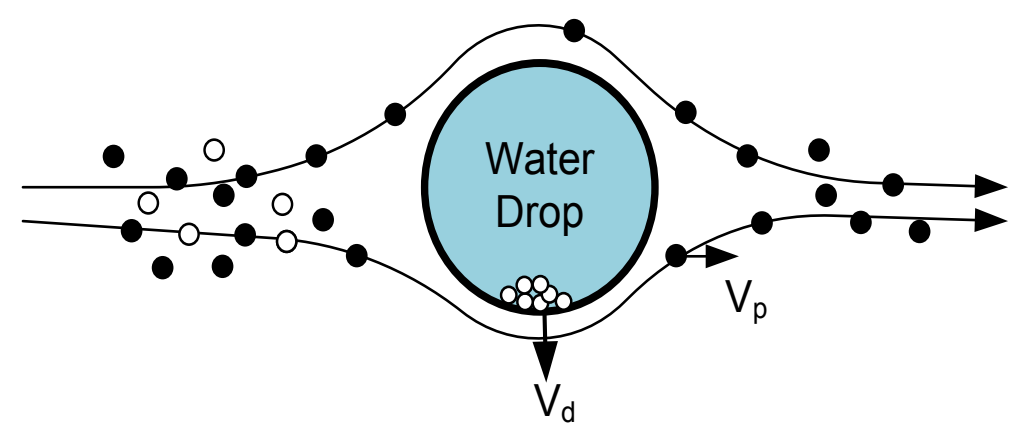

(b)

Figure 1.1: Comparison between (a) conventional flotation where particles in water interact with rising air bubbles and (b) the first approach investigated in the present study where falling drops interact with particles suspended in air. $\mathbf{O}=$ hydrophobic particles; $\mathrm{O}=$ hydrophilic particles.

Because of the relatively high viscosity of water, light weight ultra-fine particles do not easily attach to air bubbles. This is due to the small mass of these fine particles; they lack the required momentum to disengage from the fluid flow, and collide with the air bubbles. Even when they do collide, the momentum of these particles may be insufficient to rupture the liquid film to establish a stable three phase contact line. In addition, these particles have high specific areas, and hence require a significant bubble surface flux. Similarly, the particles exhibit a high adsorption capacity, and hence require higher concentrations of chemical reagents. Thus, the recovery of ultra-fine particles can be very low (Shahbazi et al., 2010). 
In the present study, drops of water settle through a gaseous dispersion of dry particles, colliding and engulfing the hydrophilic particles in the feed, ideally leaving behind the hydrophobic particles. Hydrophilic particles can pass directly through the gas-liquid interface and into the falling drops, whereas hydrophobic particles should either adhere with the gas-liquid interface or remain within the gas phase, as shown in Figure 1.2.

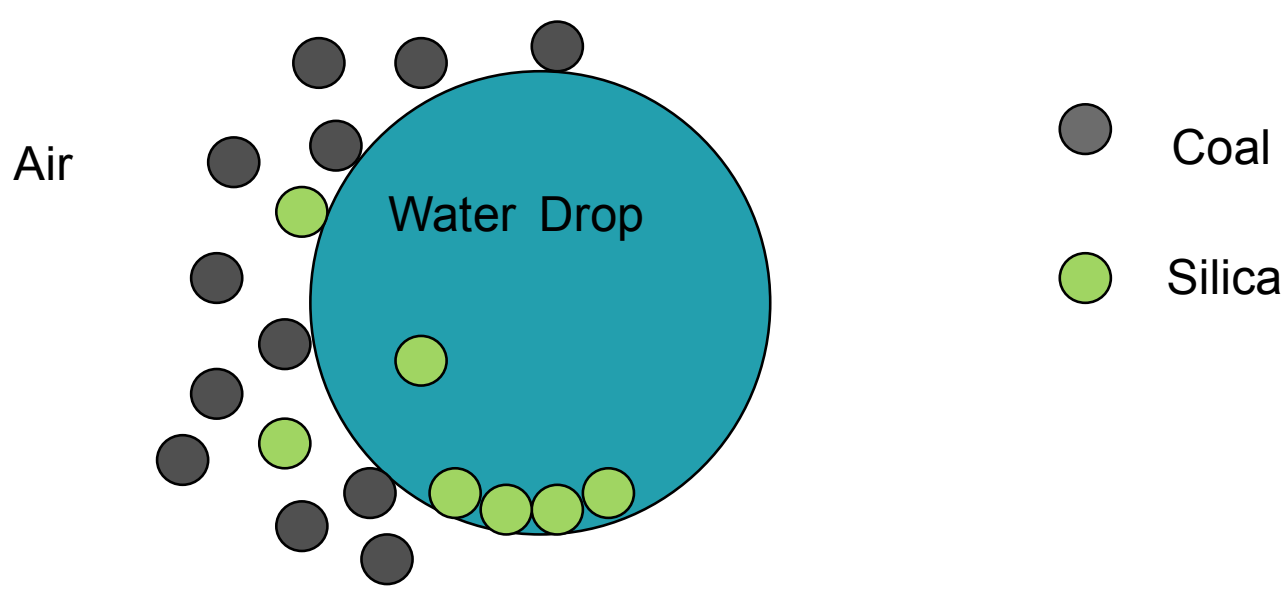

Figure 1.2: Schematic representation of the engulfment of fine silica particles into a water drop through the air-water interface.

A detailed laboratory scale experimental system was developed to investigate the feasibility of this first approach for selectively collecting fine hydrophobic particles. This system mainly consisted of a feed column, water distribution chamber and a collision chamber. In this study, a vibrated sieve was used to disperse the fine particles allowing much higher concentrations of particles to fall as a plume. The plume was drawn downwards and then into a horizontal flow using a vacuum at a sufficient rate. The gas-dispersed particles were conveyed into the collision chamber by the air flow, while the water drops fell through the dispersion. The dispersion then passed to a cyclone, allowing fine particle recovery via the cyclone underflow, while the overflow passed through a bag filter to recover the remaining, ultrafine, particles. The challenges involved in the development of this system, right through to the final system design, and performance are outlined in Chapter 5. 


\subsection{Second Approach - Electrostatic Transport of Particles}

An alternative approach involving electrostatic forces was developed and investigated for the first time in this study. Electrostatic scrubbers use oppositely charged fine particles and water drops to collect particles in to the drops. Under this study a simplistic approach was taken to investigate the collection of charged particles in to a hanging drop.

Electrostatic forces are especially attractive due to their application over significant distances, certainly in comparison to the distances involved when van der Waals and other surfaces forces are applied. However, these forces can lead to poor reproducibility due to difficulties in harnessing the force.

Thus much of the research concerned with the application of electrostatic forces was focussed on developing the protocols required in order to achieve reproducibility. A sudden loss in charge can occur for no apparent reason in these systems, thus a comprehensive series of experiments was needed to confirm the essential requirements. Once established, it was possible to undertake more precise experiments based on the separation distance and the rate of change of the separation distance with time. Experiments were also undertaken using different particle species, and liquids ranging from water, to highly viscous glycerine, to a pure hydrocarbon capable of exhibiting only dispersive interactions.

Simple experiments were performed in which a charged substrate with a bed of fine hydrophilic and hydrophobic particles was raised towards a suspended liquid drop (Figure 1.3). Video images were used to analyse the drop-particle interactions. Full details of this study are given in Chapter 7. 


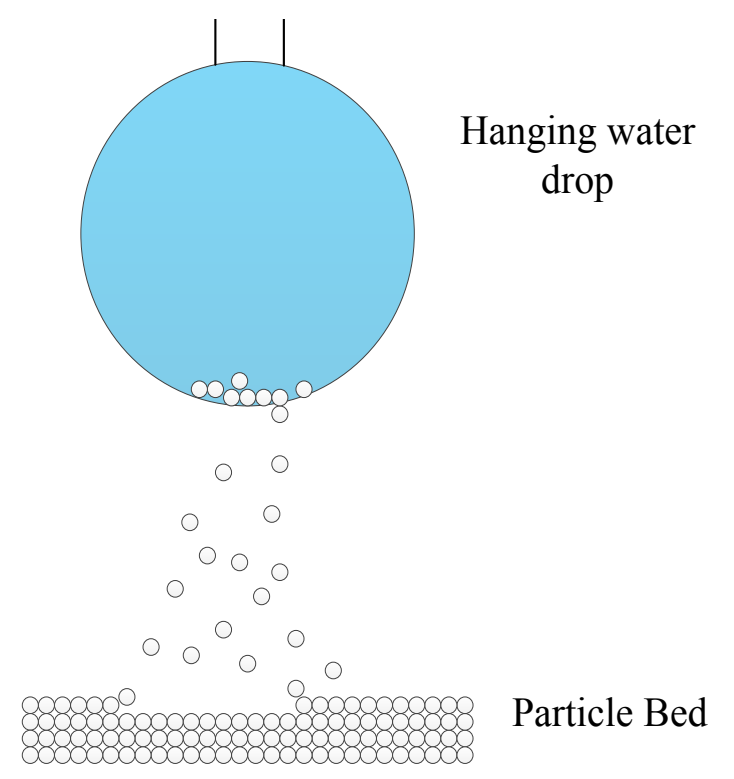

Figure 1.3: Schematic of transfer of electrostatically charged glass ballotini to a hanging pendent water drop.

\subsection{Aims and Objectives}

The main objective of this Thesis is to explore the process of fine particle collisions with drops of water, and in turn assess the potential selectivity between hydrophobic and hydrophilic particles. This work involved two different approaches. The first approach involved a dispersion of fine particles conveyed by air through a zone of falling drops. The second approach involved the application of electrostatic forces to convey the particles towards a single drop.

A key focus of the first approach was the development of a suitable laboratory system for controlling the dispersion of particles and forming the system of falling drops. A simple mathematical model of the particle-drop collision was developed to assist with the interpretation of the results.

In the second approach, a novel method was developed to harness the electrostatic force, and in turn energize the particles, delivering them to a specific drop of water. Given the novelty of this system, considerable work was involved in establishing the rules governing the reproducibility, the charge transfer rates, and the particle behaviour within the drop. 


\subsection{Thesis Outline}

This Thesis begins with a literature review in Chapter 2 related to the thermodynamics and kinetics of particle adhesion. The theoretical framework discussed in this chapter explains the surface forces, solid-liquid interfaces, the concept of contact angle and particle charging mechanisms in electrostatics.

Chapter 3 consists of a comprehensive review of fundamentals of particle-bubble and particle-drop interactions and their applications. A simple mathematical model of particle-drop collision is proposed in Chapter 4. Chapter 5 describes the laboratory scale system developed for the first approach, involving a dispersion of particles conveyed by air through falling drops The results obtained in these experiments are presented and discussed in Chapter 6. Chapter 7 explains the electrostatic approach of selective collection, and the results obtained. Chapter 8 concludes this Thesis with a discussion and recommendations arising from this research. 


\section{Chapter 2}

\section{FORCES AND PHENOMENA INVOLVED IN PARTICLE- LIQUID-GAS INTERACTIONS}

\subsection{Introduction}

\subsection{Surface Forces}

2.2.1 van der Waals Interactions

\subsubsection{Electrostatics Forces}

2.2.3 Classical DLVO Theory of Surface Forces

\subsubsection{Extended DLVO Theory}

2.2.4.1 Non DLVO Forces

2.2.4.2 Hydrophobic Phenomena

\subsection{Wetting and The Concept of Contact Angle}

\subsubsection{Wetting}

2.3.2 The Concept of Contact Angle

\subsubsection{Young's Equation}

2.3.4 Wetting and Adhesion

2.3.4.1 The Work of Cohesion $\left(W_{\mathrm{c}}\right)$

2.3.4.2 The Work of Adhesion $\left(W_{\mathrm{A}}\right)$

2.3.5 Young-Laplace Equation 


\subsection{Surface Energy and Spreading}

2.4.1 Surface Energy

2.4.2 Spreading

2.4.3 Spreading Co-efficient $(S)$

\subsection{Dynamic Contact Angles and Kinetics of Wetting}

2.5.1 Contact Angle Hysteresis

2.5.2 Dynamic Contact Angles

2.5.3 Kinetics of Wetting

2.5.3.1 Hydrodynamic Model of Kinetics of Wetting

2.5.3.2 Molecular Kinetics Theory of Wetting

2.5.3.3 Combined (Microhydrodynamic) model

\subsection{Electrostatics}

2.6.1 Introduction to Electrostatics

2.6.2 Conductors and Non Conductors (Dielectric Particles/Insulators)

2.6.3 Mechanisms of Particle Charging

2.6.3.1 Induction

2.6.3.2 Conduction

2.6.3.3 Tribocharging (Triboelectrification/Friction Charging)

2.6.4 Effects of Physical Conditions

\subsection{Summary}




\subsection{Introduction}

An understanding of the wetting and adhesion between a particle and a drop is essential in developing the selective collection concept. Particle adhesion is mainly controlled by surface interactions such as surface forces, surface energy and surface charges.

Surface chemistry has been built upon significant fundamental research contributions over more than two centuries. Thomas Young (1804) and Gibbs (1873) laid the foundation for understanding the interfacial chemistry of adhesion. Since then many scientists have contributed to building the theories of colloid and interfacial science, which have then been applied to the mineral processing industries. This chapter reviews the fundamental physicochemical phenomena that underpin the thermodynamics of wetting and particle adhesion. The present research has also investigated an alternative method of particle collection through electrostatics. Hence, at the end of this chapter, literature relevant to electrostatics has also been discussed.

\subsection{Surface Forces}

Figure 2.1 shows the mutual attraction between adjacent molecules comprising a homogeneous liquid. Within the liquid bulk, the interactions are cohesive in nature, with the interactions being similar in all directions. However, at the surface, molecules experience a net inwards force, which in turn manifests a tension or, in effect, an excess energy at the surface.

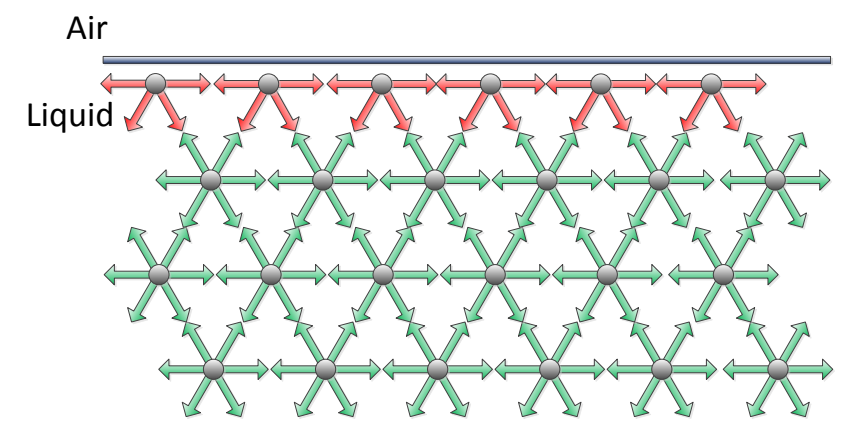

Figure 2. 1: Origin of surface tension forces acting at a liquid surface. 
The surface forces between solid-liquid and solid-gas interfaces play a very important role in fine particle fluid interactions. Since fine particles have high surface area to mass ratios, surface forces dominate their behaviour. There are various different surface forces (short range forces) that can exist, such as van der Waals, electrostatic forces, capillary forces, steric forces and longer range forces such as the hydrophobic force. The main surface forces and the factors affecting their magnitudes will be discussed in this section. The nature and magnitude of the surface forces that operate at the molecular level is determined by the properties of the molecules, electronic distribution and ionic charge (Rao \& Leja, 2006).

\subsection{1 van der Waals Interactions}

The van der Waals force is a short range electromagnetic force between two molecules or atoms that arises due to the attractive electromagnetic force between opposite polarisations (Masuda et al., 2007). This intermolecular or intramolecular force can occur between particles as well as between a particle and a bubble/drop. Further, the van der Waals attraction is usually greater than the electrical double-layer repulsion (electrical double layer forces will be discussed in the following section) at small distances (Rao \& Leja, 2004). The polarised molecules have a weak van der Waals force in between oppositely polarised ends as shown in Figure 2.2.

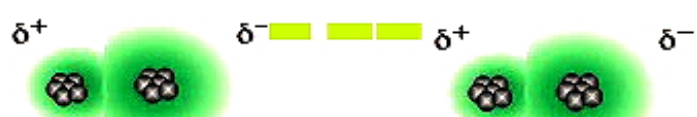

Figure 2.2: van der Waals interactions between two dipolar molecules.

van der Waals (Erbil, 2006) formulated the following equation in 1873 for non-ideal gases.

$$
\left(P+\frac{a n^{2}}{V^{2}}\right)(V-n b)=n R T
$$


where $P$ is the external pressure $(\mathrm{Pa}), V$ the molar volume $\left(\mathrm{m}^{3}\right), R$ the ideal gas constant $(\mathrm{R}=8.3145 \mathrm{~J} / \mathrm{Kmol}), T$ the absolute temperature $(\mathrm{K})$, $a$ the attraction constant for molecules $\left(\mathrm{Pa} \mathrm{m}^{6} / \mathrm{mol}^{2}\right), b$ the actual volume of the molecules $\left(\mathrm{m}^{3} / \mathrm{mol}\right)$ and $n$ the number of moles. This equation accounts for attractive intermolecular forces.

The van der Waals forces operate over a typical range of $0.2-15 \mathrm{~nm}$ (Israelachvili, 2011; Erbil, 2006; Butt et al., 2003). There are three types of van der Waals interactions:

I. rotating dipole-dipole (permanent dipole) interactions (Keesom)

II. permanent dipole-induced dipole interactions (Debye)

III. induced dipole interaction (London-van der Waals or dispersion forces)

The Keesom and Debye forces need permanent dipoles in the system. In contrast, the London dispersion forces are caused entirely by temporary induced dipoles. Temporary dipoles are formed from random fluctuations in electron density. These in turn induce aligned dipoles in neighbouring molecules, causing attraction between them. The dispersion force is inversely proportional to the sixth power of distance between the particles (i.e. $E \propto-1 / D^{6}$ where $E$ is the dispersion force and $D$ the center-center distance between particles).

The London dispersion force is the main contributing van der Waals force for the attachments (adhesion and wetting) that occur in flotation. The London van der Waals force between similar particles in a medium is always attractive. This attractive force between neutral non-polar molecules is the cause of fine particle aggregation. In the case of dissimilar particles such as particle-bubble interactions, the van der Waals force becomes repulsive. The van der Waals interaction energy can be calculated by either the Hamaker approach or by the Lifshiftz approach. The sign and the value of particle interaction can be expressed in terms of the Hamaker constant (Masuda et al., 2007; Erbil, 2006; Nguyen \& Schulze, 2003; Mao, 1998). 


\subsubsection{Electrostatic Forces in Air}

When charged particles are in air, electrostatic forces arise due to particle-charge interactions, image-charge interactions or electrostatic contact potential differences (Masuda et al., 2007). The electrostatic force is considerable for particles less than 45 microns in diameter.

Dissimilar particles which can be electrically charged by rubbing were studied in early 600 BC by the Greeks (Kelly \& Spottiswood, 1988). In 1785 Coulomb showed that the force $(F)$ between two isolated small charges $\left(q_{1}\right.$ and $\left.q_{2}\right)$ is given by,

$$
F=\frac{1}{4 \pi \epsilon} \frac{q_{1} q_{2}}{D^{2}}
$$

where $D$ is the distance between the charges and $\varepsilon$ is known as the permittivity or dielectric constant of the medium between the charges.

This force is measured by Newtons per coulomb (N/C). Permittivity (F/m) can be expressed in terms of permittivity of the vacuum $\left(\varepsilon_{0}\right)$ as $\varepsilon=\varepsilon_{r} . \varepsilon_{0} . \varepsilon_{r}$ is the relative permittivity or relative dielectric constant. When two equi-size charged particles are in close proximity and the gap between the particles is smaller than the diameter of the particle, then the force between particles can be expressed by,

$$
F=\frac{\pi \sigma_{1} \sigma_{2}}{\varepsilon_{0}} d_{p}^{2}
$$

where $\sigma_{1}$, and $\sigma_{2}$ are the surface charge densities of the two particles and $d_{p}$ is the particle diameter. If the charges are of the same sign, they will repel each other; if of opposite sign, they will attract.

By definition, the force $(F)$ acting on a charge $(q)$ in an electric field of strength $E$ (N/C) is,

$$
F=q . E
$$


In the real world, electric charges are distributed over the surface of irregular particles, leading to non-uniform electric fields. In addition, with finite sizes of the particles and with different dielectric permittivities from that of the medium, the local electric fields can be altered significantly.

\subsubsection{Classical DLVO Theory of Surface Forces}

When a particle and a bubble are close to each other, an intervening liquid film forms and the stability of this film can be explained using surface force concepts of DLVO theory. Derjaguin and Landau (1941) and Verwey and Overbeek (1948) have investigated the classical DLVO theory, which combined the electrostatic repulsive forces and attractive van der Waals forces (Verwey \& Overbeek, 1948; Derjaguin et al.,1987). As per the DLVO theory, the stability of the colloidal suspension depends on the total energy $\left(V_{t}\right)$,

$$
\mathrm{V}_{\mathrm{t}}=\mathrm{V}_{\mathrm{e}}+\mathrm{V}_{\mathrm{d}}
$$

where $V_{e}$ is the repulsive electrostatic energy and $V_{d}$ the attractive London van der Waals energy.

In the case of bubble-particle attachment, the molecular force is repulsive compared to the particle-particle attachment force. When the bubble and particle are unequally charged, electrostatic repulsion turns into attraction at a very short bubble-particle distance. Figure 2.3 shows the change of these force components with respect to distance between the two surfaces. As per this graph, the attractive van der Waals force increases up to a short distance while the repulsive electrical double layer force decreases exponentially with the increasing distance between surfaces. By adding these two opposite forces DLVO theory can predict the net force acting on a particle approaching to a bubble or another particle. The net force/ energy barrier increases with the decreasing of the distance between the surfaces. Also at a particular distance, $\mathrm{V}_{\mathrm{e}}$ exceeds $\mathrm{V}_{\mathrm{d}}$. 

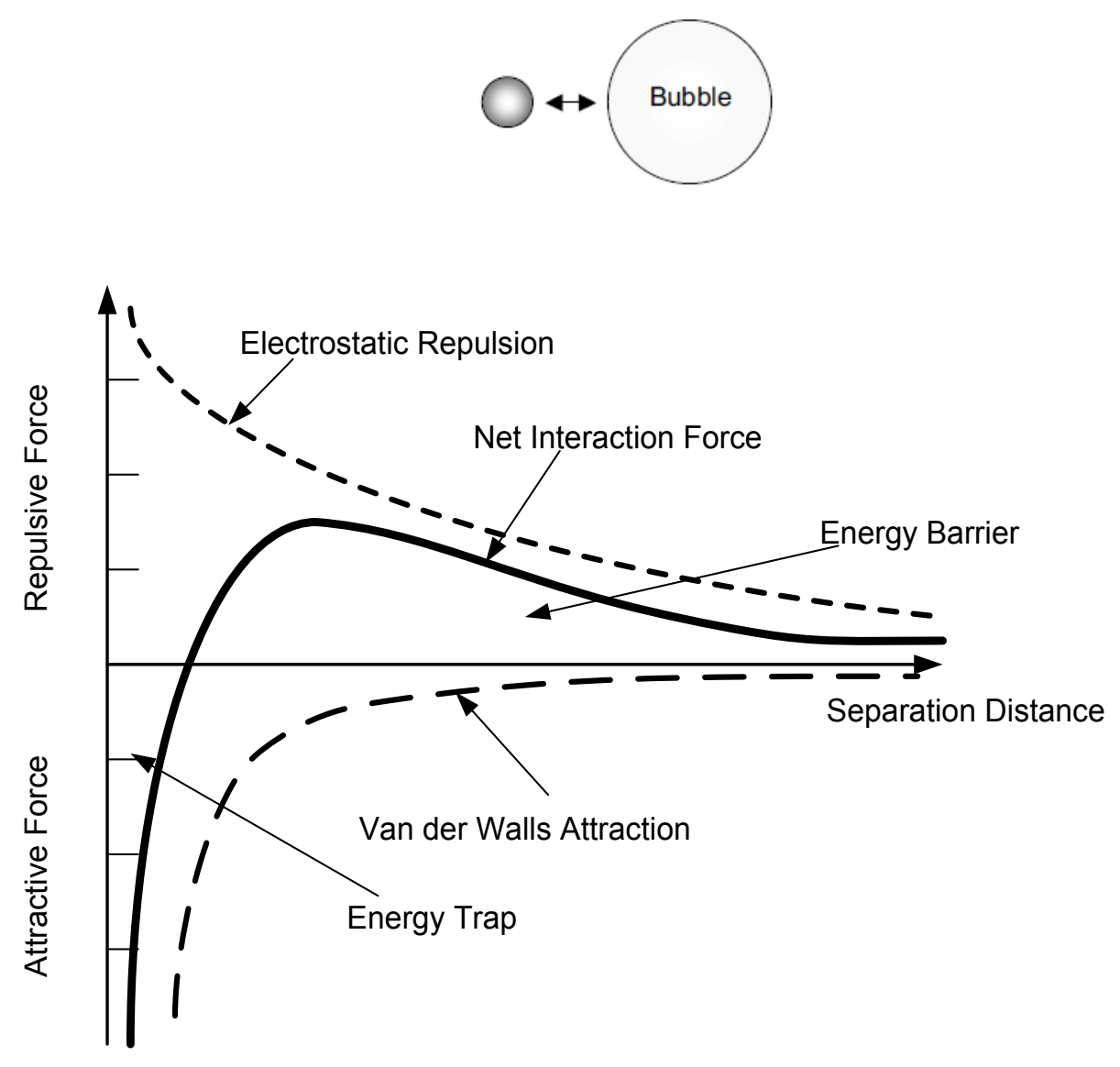

Figure 2.3: Interaction energy between a particle and a bubble as per the DLVO theory

Further, the van der Waals attraction is greater than the electrical double-layer repulsion at small distances as it is a power-law interaction ( i.e $\mathrm{E} \alpha-1 / \mathrm{D}^{\mathrm{n}}$ ), compared to the double layer interaction. The electrostatic energy remains finite or rises more slowly as the separation distance (D) reaches zero. Hence, this large energy barrier prevents the attachment of the bubble and particle in flotation.

To overcome this problem Mao and Yoon (1996) suggested that DLVO theory is applicable only to particles that are neither hydrophobic nor hydrophilic. Therefore, they extended the DLVO theory by adding a third force. This is called the extended DLVO theory. 


\subsubsection{Extended DLVO Theory}

$\mathrm{Xu}$ and Yoon (1989) extended the DLVO theory by adding a structural force $\left(\mathrm{V}_{\mathrm{s}}\right)$. The extended DLVO theory gives the total energy as,

$$
\mathrm{V}_{\mathrm{t}}=\mathrm{V}_{\mathrm{e}}+\mathrm{V}_{\mathrm{d}}+\mathrm{V}_{\mathrm{s}}
$$

$\mathrm{V}_{\mathrm{s}}$ shows how surfaces and colloids coalesce rapidly. The origin of the structural force still remains unresolved. This force is a non DLVO force. When the distance between two surfaces is too short, DLVO forces fail to explain the interaction between them. Hence, it is believed that non DLVO forces play a role at this proximity. These forces can be repulsive or attractive and stronger than other DLVO forces. There are two main non-DLVO forces such as Structural forces and Steric forces. Structural forces consist of hydration and hydrophobic forces. Hydrophobic forces will be discussed later.

\subsubsection{Non DLVO Forces}

When the distance between the particles is less than a few nanometres, continuum theories of van der Waals and electrostatic forces fail to explain their interaction (Israelachvili, 1992). This can be due to the other forces called "non DLVO forces". There are two main non DLVO forces, namely Structural forces and Steric forces. Since these forces are strong over a short range they are particularly important in particle adhesion.

Structural forces act within a water medium. Structural forces consist of hydration and hydrophobic forces. Such forces arise when the presence of a surface alters the nature of the fluid. Hydration forces are strong forces acting on polar (hydrophilic) surfaces in a water medium while hydrophobic forces arise due to hydrophobic surfaces, which repel water molecules. An example of a naturally hydrophobic surface is coal. Steric forces arise due to overlapping of adsorbed layers of two particles. At very small interatomic distances, the electron clouds of atoms overlap, and arises a strong repulsive steric force 
that determines how close two atoms or molecules can ultimately approach each other.

\subsubsection{Hydrophobic Phenomena}

Hydrophobic particles suspended in water attract each other due to forces that are larger than the van der Waals forces, a process generally referred to as the hydrophobic interaction (or force). The hydrophobic force arises when a surface is incapable of forming hydrogen bonds with polar molecules (water). This situation can be artificially induced by adsorbed hydrophobic species (Somasundaran et al., 2009).

When a non-polar (hydrophobic) compound is mixed with water, water molecules restructure around the non-polar solute (Rao \& Leja, 2004). The orientation of water molecules in contact with a hydrophobic molecule is thermodynamically unfavourable, therefore two such molecules tend to come together simply by attracting each other. Hence non-polar (hydrophobic) molecules dissolved in water usually exhibit strong intermolecular attraction for each other in spite of them usually having a low interaction e.g. as measured by their surface tension and vapour pressure. This is called a hydrophobic bond.

Laskowski and Kitchener (1969) predicted the existence of a third force other than the van der Waals and electrostatic forces that should exist between a bubble and a particle. The hydrophobic interaction between surfaces has been recognised by Israelachvili and Pashley (1982) with mica surfaces covered by compounds with long chain hydrocarbon. They found that the hydrophobic interaction between surfaces acts over a much longer range than the van der Waals force.

Therefore it is said that hydrophobic attraction is much stronger than van der Waals attraction and it decays exponentially with the increase in separation distance. This explains rapid coagulation of hydrophobic particles in water. The magnitude of the hydrophobic attraction depends on the hydrophobicity of the substance. Hydrophobicity is characterized by the chemical composition of the mineral and bonding type.

Many investigators have observed some electrostatic contributions to the hydrophobic 
force (Claesson, Blom, Herder, \& Ninham, 1986; Christenson, Claesson, Berg, \& Herder, 1989). Tsao et al. (1991) have measured the hydrophobic force at different temperatures and have suggested that the hydrophobic force decreases with increasing temperature due to disordered monolayers. By changing the hydrophobic force it is possible to enhance the selectivity of flotation (Mao, 1998). However, there is significant ambiguity and debate about the origin and nature of hydrophobic force (Israelachvili, 2011; Churaev, N.V. 2005; Ruckenstein \& Churaev, 1991; Eriksson et al., 1989; Israelachvili and Pashley,1982)

\subsection{Wetting and The Concept of Contact Angle}

\subsubsection{Wetting}

Wetting is the ability of a liquid to spread on a solid surface, and hence is a key condition governing particle attachment in processes such as flotation. The wetting phenomenon is a result of intermolecular interactions and the associated balance between adhesive and cohesive forces, which can be interpreted in terms of the socalled contact angle, $\theta$. Figure 2.4 defines the contact angle formed by the liquid surface with the solid surface.

The degree of wetting can be described in terms of three cases (Starov et al., 2007). These are complete wetting, partial wetting (Figure 2.4(a)), and non-wetting (Figure 2.4(b)). If the contact angle between the solids and liquid is $0^{\circ}$, then this is referred to as perfect or complete wetting. This condition is caused by a strong solid-liquid interaction. When the interfacial tension between the solid and gas is less than the sum of the interfacial tensions of the liquid with the other two phases (i.e. $\gamma_{s g}<\gamma_{s l}+\gamma_{l g}$ ), a droplet with a finite non-zero contact angle minimises the free energy of the system, resulting in partial wetting. Non-wetting happens when the contact angle reaches nearly 180 degrees.

Liquids which exhibit poor wetting or high contact angles $\left(90^{\circ}<\theta<180^{\circ}\right)$ form droplets while good wetting liquids spread out over the solid surface. Water wettable surfaces are referred to as hydrophilic and non-wettable surfaces as hydrophobic. 
Highly hydrophobic surfaces have contact angles larger than 120 degrees (Zisman, 1964).

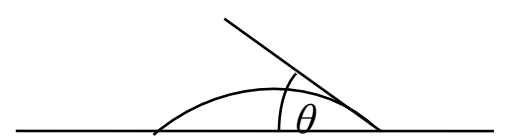

(a) Partial wetting

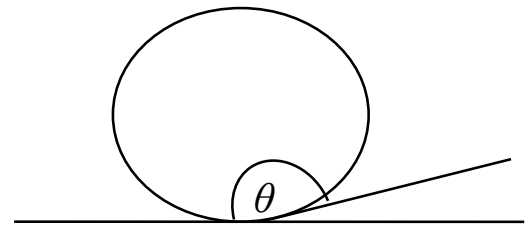

(b) Non-wetting

Figure 2.4: Contact angles for (a) partial wetting (b) non-wetting.

For a liquid to completely wet a surface the surface energy of the solid must be high and the surface energy of the liquid must be low. The surface tension of water can be lowered by adding chemicals such as surfactants, which reduces the free energy of the liquid.

Newly formed surfaces have a strong tendency to adsorb other molecules and hence to experience a reduction in surface energy with time. Roughness and heterogeneity of the solid material also affect the wetting process. Differences in wettability between surfaces that are similar in structure are due to differences in packing of the atoms and differences in the chemical nature of the materials (Rao \& Leja, 2004).

\subsubsection{The Concept of Contact Angle}

The contact angle defines the basic thermodynamic criterion for a droplet in equilibrium with a solid surface, as shown in Figure 2.5 (Chau, 2009). The contact angle is defined by the angle between the liquid-gas and the liquid-solid interfaces, measured through the liquid as shown in Figure 2.5. 


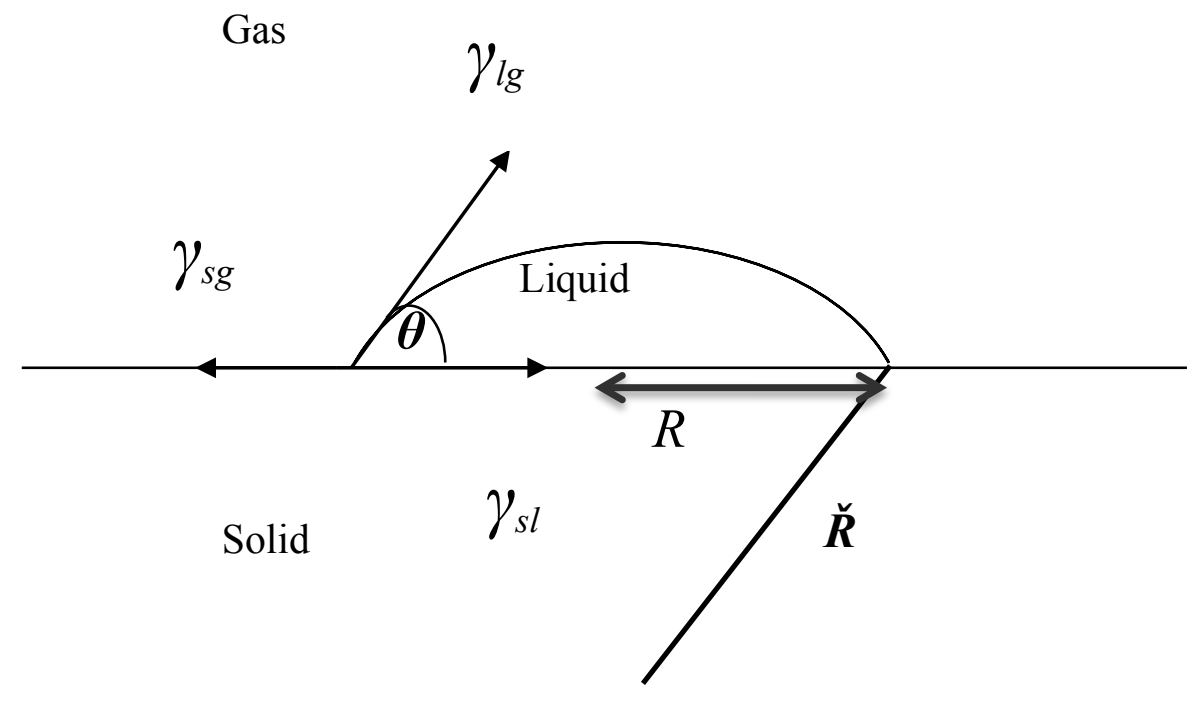

Figure 2.5: Equilibrium contact between a liquid drop and a solid in a gas. The three phase contact line is the location, where the liquid, vapour and solid phases meet. $R$ is the radius of the liquid footprint on the solid surface and $\check{R}$ is the radius of curvature of the gas-liquid interface.

The contact angle indicates the affinity between the liquid and the solid (Nicol, 1992). Most practical contact angles are in the range 0 to $90^{\circ}$ and in everyday parlance it is often only surfaces with contact angles at the higher end of this range (more than 90 degrees) that are referred to as hydrophobic, and surfaces with relatively small contact angles (less than $90^{\circ}$ ) are described as hydrophilic. As shown in Figure 2.4(a), a low value of the contact angle $(\theta)$ indicates that the liquid spreads, or wets well, while a high contact angles indicate poor wetting.

\subsubsection{Young's Equation}

Thermodynamic equilibrium of the three-phase contact angle is expressed by Young's equation, where $\gamma$ represents the interfacial tensions (or surface energies) of the relevant interfaces. This equation can also be described in terms of a horizontal force balance, between the interfacial forces. Thus the vector summation of the surface tensions at the three phase contact point gives (Young, 1805), 


$$
\cos \theta=\frac{\gamma_{s g}-\gamma_{s l}}{\gamma_{l g}}
$$

where $\gamma_{s g}$ represents interfacial tension between solid-gas, $\gamma_{s l}$ represents interfacial tension between solid-liquid, and $\gamma_{l g}$ represents interfacial tension between liquid-gas.

Young's equation can also be derived from thermodynamic considerations. The change in surface free energy $(\Delta \mathrm{G})$ of a droplet spreading over a solid surface is shown in Figure 2.6 in terms of the increase in the wetted area $\Delta A$.

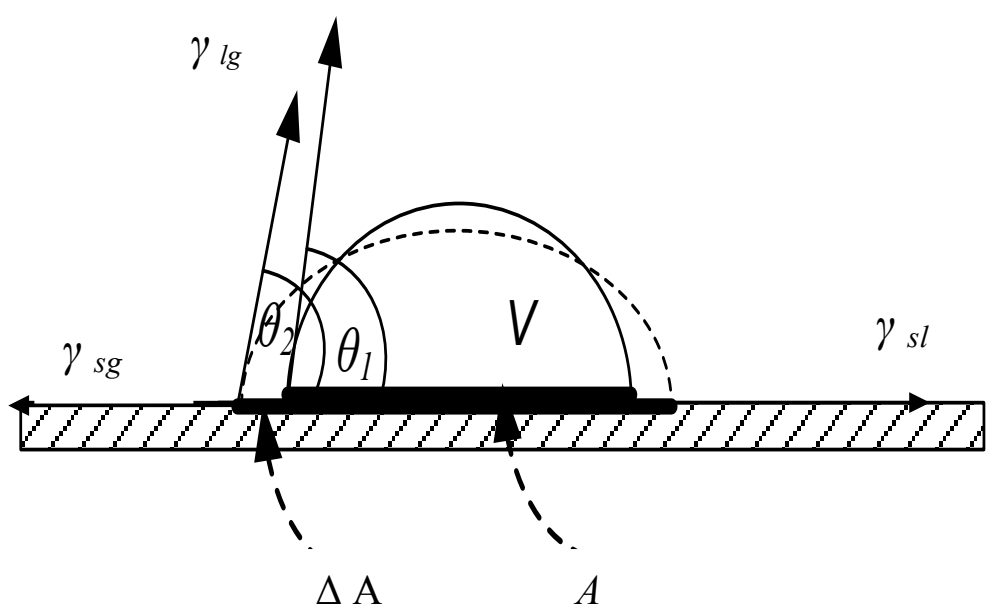

Figure 2.6: Droplet spreading on a solid surface.

The drop volume $(V)$ is constant. Assuming negligible change in potential energy, the change in free energy can be expressed as (Roura \& Fort, 2004),

$$
\Delta G=\Delta A\left(\gamma_{s l}-\gamma_{s g}\right)+\Delta A \gamma_{l g} \cos (\theta-\Delta \theta)
$$

$\Delta \theta$ is the change of the contact angle $\left(\theta_{1}-\theta_{2}\right)$ resulting from the spreading. At equilibrium, the change in free energy with increasing interfacial solid-liquid area goes to zero, leading directly to Young's equation.

Though this derivation is for a flat surface; the contact angle is independent of the surface geometry. Hence this angle is the same for a drop on a curved surface. Also, the 
result is independent of the nature of the forces between the molecules of the phases so long as these are of shorter range than the dimensions of the droplet (Israelachvili, 1992).

Young's equation (Eq. 2.7) gives a relationship between the surface energies, which can be used to calculate the work of adhesion between particles and drops.

\subsubsection{Wetting and Adhesion}

Adhesion develops from physical bonds (e.g. weak van der Waals), chemical interactions, and friction from irregular surface topography (Asthana \& Sobczak, 2000). When there is a high work of adhesion, it indicates good wetting, whilst a low work of adhesion indicates poor wetting. Most liquids will completely wet high-energy surfaces. Low-energy surfaces primarily interact with liquids through dispersion (van der Waals) forces. Zisman (1964) observed that $\cos \theta$ increases linearly as the interfacial tension, $\gamma_{l g}$, of the liquid decreases.

\subsubsection{The Work of Cohesion $\left(W_{\mathrm{c}}\right)$}

The work of cohesion is the energy required to create two new interfaces within a single bulk phase. Hence it can be defined as $W_{\mathrm{c}}$ for a reversible process (Good, 1992) of separating a unit cross sectional area within a continuous phase to form two surfaces as illustrated in Figure 2.7.

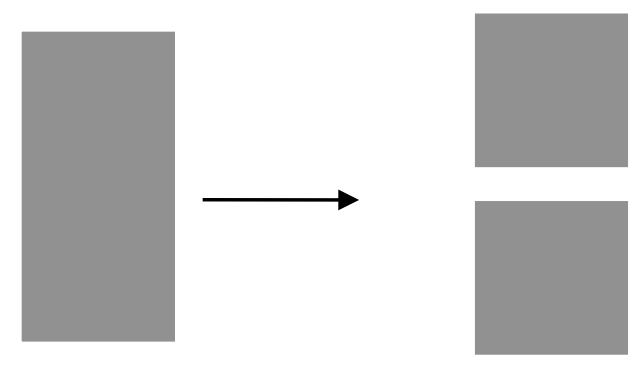

Figure 2.7: Diagram of cohesion process.

$$
-\Delta G / A=W_{c}=2 \gamma
$$


The work of cohesion is a specific energy defined as two times the surface energy per unit area $(A)$ of the newly formed surfaces.

\subsubsection{The Work of Adhesion $\left(W_{\mathrm{A}}\right)$}

The work of adhesion $\left(W_{\mathrm{A}}\right)$ refers to the free energy change per unit area that occurs when two different materials, joined at an interface, are separated reversibly and isothermally (Erbil, 2006). Work of adhesion is the work done per unit area at constant pressure and temperature to separate to infinity a column of two phases (different liquids or solids) creating two new equilibrium surfaces of two pure materials at the new interface as shown in Figure 2.8. Hence:

$$
W_{A}=-\Delta G / A=\gamma_{\mathrm{A}}+\gamma_{\mathrm{B}}-\gamma_{\mathrm{AB}}
$$

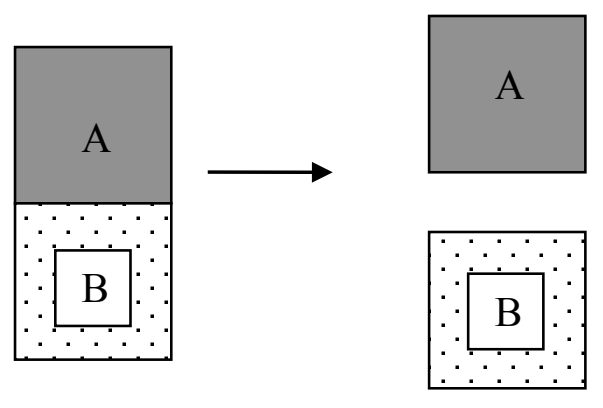

Figure 2.8: Diagram of adhesion process.

In conventional froth flotation the attachment or adhesion of particles to an air bubble is shown in Figure 2.9.
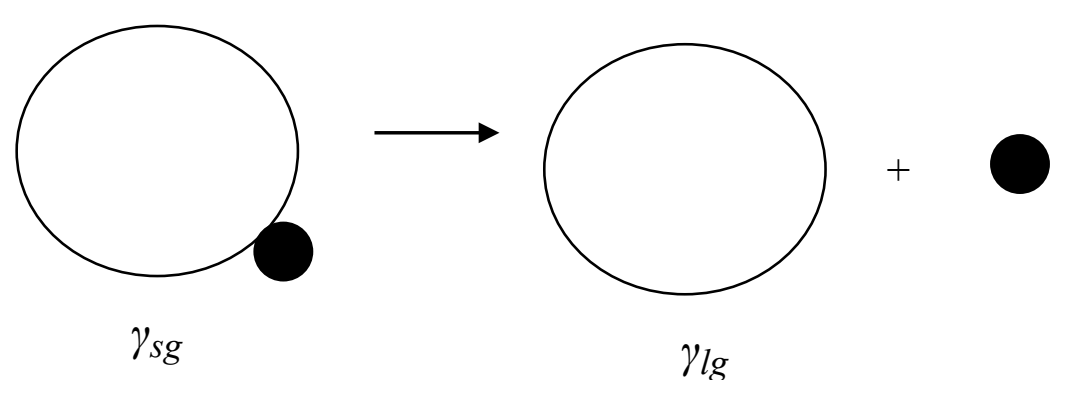

Figure 2.9: Work of adhesion between a particle and an air bubble in conventional flotation. 
As illustrated in Figure 2.9, the attachment of a particle to a bubble occurs via the displacement of water by air on the solid surface. Hence, the free energy change for particle-bubble attachment in terms of the work of adhesion is given by the following equation.

$$
\Delta \mathrm{G}_{s g}=-W_{A, s g}=\gamma_{s g}-\gamma_{s l}-\gamma_{l g}
$$

By combining Young's equation (Eq. 2.7) with the work of adhesion for solid-liquid $\left(W_{s l}\right)$ interfaces (Eq. 2.11), Dupré (1869) derived the following equation, known as the Young- Dupré equation:

$$
-\Delta G_{s l}=W_{A, s l}=\gamma_{l g}(1+\cos \theta)
$$

The effect of wettability on interfacial adhesion can be quantified by this equation. An increase in the contact angle indicates a decrease in the free energy change. When the contact angle is small, the work of adhesion is high and considerable energy must be spent to separate the solid from the liquid. If $\theta=0, W_{s l}=2 \gamma_{l g}$, if $\theta=90^{\circ}, W_{s l}=\gamma_{l g}$ and if $\theta=180^{\circ}, W_{s l}=0$ which means no work is required to separate the liquid from the solid surface.

\subsubsection{The Young-Laplace Equation}

The Young-Laplace equation provides a mechanical force balance between the forces that arise from the interfacial tension and the surface curvature. 


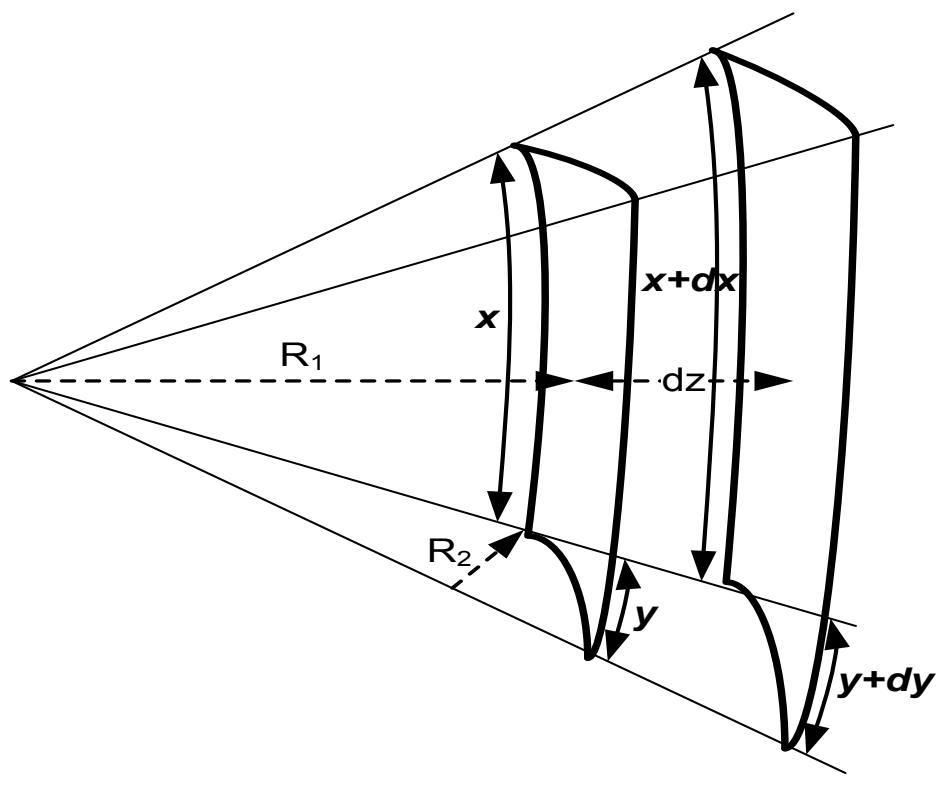

Figure 2.10: Surface curvature and pressure.

Consider a section of surface small enough so that both $R_{1}$ and $R_{2}$ are essentially constant as shown in Figure 2.10. If the surface is displaced by a distance $d z$ outward, the change in the area is given by:

$$
\Delta A=(x+d x)(y+d y)-x y=x d y+y d x
$$

The work done to form this additional surface area is:

$$
W=\gamma(x d y+y d x)
$$

This work can also be calculated from the pressure force exerted over the area multiplied by the displacement:

$$
W=\Delta P x y d z
$$

Using similar triangles,

$$
\begin{array}{r}
\frac{x+d x}{R_{1}+d z}=\frac{x}{R_{1}} \\
d x=\frac{x d z}{R_{1}}
\end{array}
$$




$$
\begin{array}{r}
\frac{y+d y}{R_{2}+d z}=\frac{y}{R_{2}} \\
d y=\frac{y d z}{R_{2}}
\end{array}
$$

From the conservation of energy it is possible to equate 2.12(a) and 2.12 (b) for the work done and stored. After substituting for $d x$ and $d y$ the following equality follows (Young \& Laplace, 1830):

$$
\Delta p=\gamma\left(\frac{1}{R_{1}}+\frac{1}{R_{2}}\right)
$$

where $\Delta p$ is the Laplace pressure and $R_{1}$ and $R_{2}$ are the principal radii of curvature (Adamson \& Gast, 1997). Therefore the pressure difference decreases to zero as the radius becomes infinite i.e. when the surface becomes flat.

For a hemi-spherical cap with radius of curvature $r=R_{1}=R_{2}$;

$$
\Delta p=\frac{2 \gamma}{r}
$$

By use of the Young-Laplace equation the degree of capillary rise can be related to the contact angle $\theta$ and surface tension $\gamma_{\text {gg }}$ of the liquid.

The pressure inside a spherical surface is always greater than the pressure outside. Young and Laplace (1806) pointed out that this capillary pressure difference between the two phases $(\Delta p)$ is proportional to the surface tension $\gamma$ and inversely proportional to the average radius curvature $R_{a v}$ of the interface. Average radii of the curvature are the mean curvature of the surface with two principal radii such as $R_{1}$ and $R_{2}$ as shown in Figure 2.10. 


\subsection{Surface Energy and Spreading}

\subsubsection{Surface Energy}

The surface energy of a system can be defined either with respect to the Gibbs energy $(G)$ or Helmholtz energy $(F)$. The Helmholtz energy is the energy obtained from a closed system, or work done on a closed system, at conditions of constant temperature and volume (Erbil, 2006). That is,

$$
F \equiv U-T S
$$

where $U$ is the internal energy of the system, $T$ the absolute temperature, and $S$ the entropy of the system.

The Gibbs energy is the non-expansion work done by a closed system. Thus,

$$
G \equiv U-T S+P V
$$

The Gibbs energy is usually more relevant than Helmholtz energy, because its natural variables, temperature and pressure, are constant in most applications. Surface energy is the increase in the Gibbs energy per increase in surface area, $A$, at constant temperature, $T$, and pressure, $P$ (Adamson \& Gast, 1960). That is,

$$
\gamma=\left(\frac{\partial G}{\partial A}\right)_{T, P}
$$

Surface energy can also be defined in terms of the surface excess energy (Peckham, 1995) per unit area, which is the energy that exists in the system over and above that which applies to the bulk phases. The surface energy of the solids depends on the chemical bonds and lattice structures (Gupta \& Yan, 2006). Leja and Poling (1960) confirmed that the contact angle is simply an indication of the system's interfacial free energy. A low surface energy material, for example coal, achieves a large contact angle, minimizing the contact area of the liquid. 


\subsubsection{Spreading}

Spreading occurs when a liquid already in contact with a solid surface flows so as to increase the solid-liquid and liquid-gas interfacial areas and decrease the solid-gas interfacial area. The spreading of a drop on a horizontal surface has been studied by many authors both experimentally and numerically (Greenspan, 1978; Chen \& Wada, 1988; Hocking, 1992; Brenner, 1993). Spreading also occurs between a liquid and the surface of another immiscible liquid. Spreading occurs mainly due to capillarity which causes the free surface to take the shape of a surface of constant curvature (Hocking, 1991).

\subsubsection{Spreading Co-efficient $(S)$}

Harkins (1952) introduced the term "spreading coefficient" $(S)$ to provide a measure of the tendency of a liquid to spread across a surface (Butt et al., 2003). The spreading coefficient of a liquid over a solid $\left(S_{L / S}\right)$ can be interpreted as the reduction in surface free energy on losing the solid/vapour and forming the new solid/liquid and liquid/vapour interfaces. It can also be called the work of spreading $\left(W_{s}\right)$, which is expressed as,

$$
W_{s}=-\Delta G_{S}=S=W_{A}-W_{C}=\gamma_{s g}-\gamma_{l g}-\gamma_{s l}
$$

Harkins' experimental results have proved (Rao, 2004) that spreading takes place whenever $S \geq 0$. For water on hydrophobic surfaces $S_{\mathrm{L} / \mathrm{S}}$ is negative. With respect to conventional flotation, the equilibrium spreading coefficient of water over the solid/gas interface should be less than zero to displace water from the zone between the particle and the bubble. 


\subsection{Dynamic Contact Angles and Kinetics of Wetting}

\subsubsection{Contact Angle Hysteresis}

If a sessile drop is placed on a flat surface, and the surface is then gradually tilted, the contact angle on the lower side of the drop will increase and the contact angle on the upper side will decrease (Figure 2.11). The 'advancing' $\left(\theta_{a}\right)$ contact angle is defined as the maximum contact angle that can be produced without increasing the interfacial area between the drop and the solid i.e. without the lower three-phase contact line starting to move downhill. The 'receding' $\left(\theta_{r}\right)$ contact angle is defined as the smallest contact angle formed by the drop without shrinking the interfacial area i.e. before the upper three-phase boundary line starts to slip downwards.

\section{Gas}

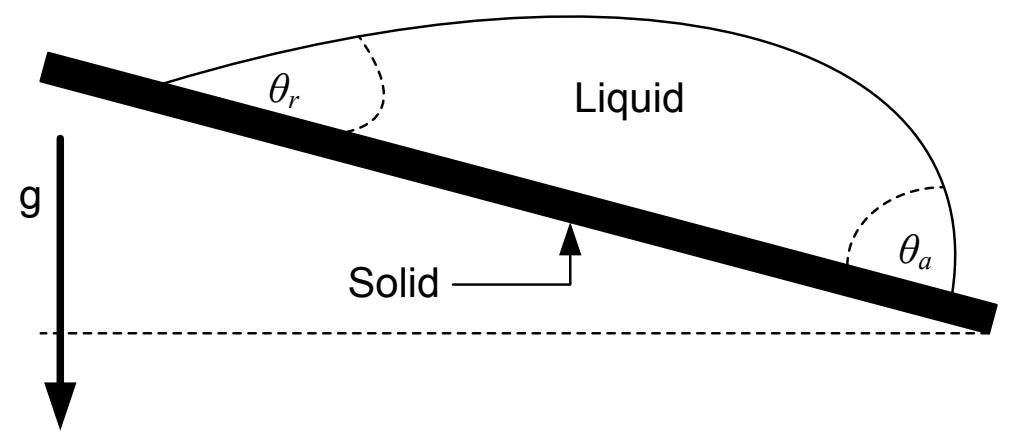

Figure 2.11: Advancing $\left(\theta_{a}\right)$ and receding $\left(\theta_{r}\right)$ contact angles.

The difference between the maximum (advanced/advancing) and minimum (receded/receding) contact angle values is called the contact angle hysteresis (Bonn et al., 2009).

$$
H=\theta_{\mathrm{a}}-\theta_{\mathrm{r}}
$$

The main reasons for hysteresis are surface roughness, surface heterogeneity, surface polarity, surface contamination and drop stability. These problems cause variations in 
the mechanical, chemical and/or thermal equilibrium. Hence hysteresis has been used to characterize surface heterogeneity, roughness and mobility. In heterogeneous surfaces, there are domains which present barriers to the motion of the contact line. These domains represent areas with different contact angles compared to the surrounding surface. When testing with water, advancing angles will be sensitive to the hydrophobic domains and receding angles will characterize the hydrophilic domains on the surface.

(Xu et al., 2009)

An ideal solid surface is perfectly flat, rigid, smooth, and chemically homogeneous and has zero contact angle hysteresis. If the hysteresis is zero, then the advancing and receding contact angles are equal. The slightest tilt of the surface leads to the sliding of the drop.

\subsubsection{Dynamic Contact Angles}

The contact angle defined by Young's equation is a static thermodynamic equilibrium value. If the three phase (liquid/solid/vapour) contact line is in motion, however, the angles produced are called dynamic contact angles. During its motion toward an equilibrium shape, a liquid droplet forms a range of dynamic (apparent) contact angles.

The dynamic angles depend upon the spreading rate (de Gennes, 1985). These contact angles change with time due to different forces acting on the liquid solid interfaces. As shown in Figure 2.12, dynamic contact angles can have a range of values above the advancing contact angle, $\left(\theta_{a}\right)$, and below the receding contact angle, $\left(\theta_{r}\right)$. 


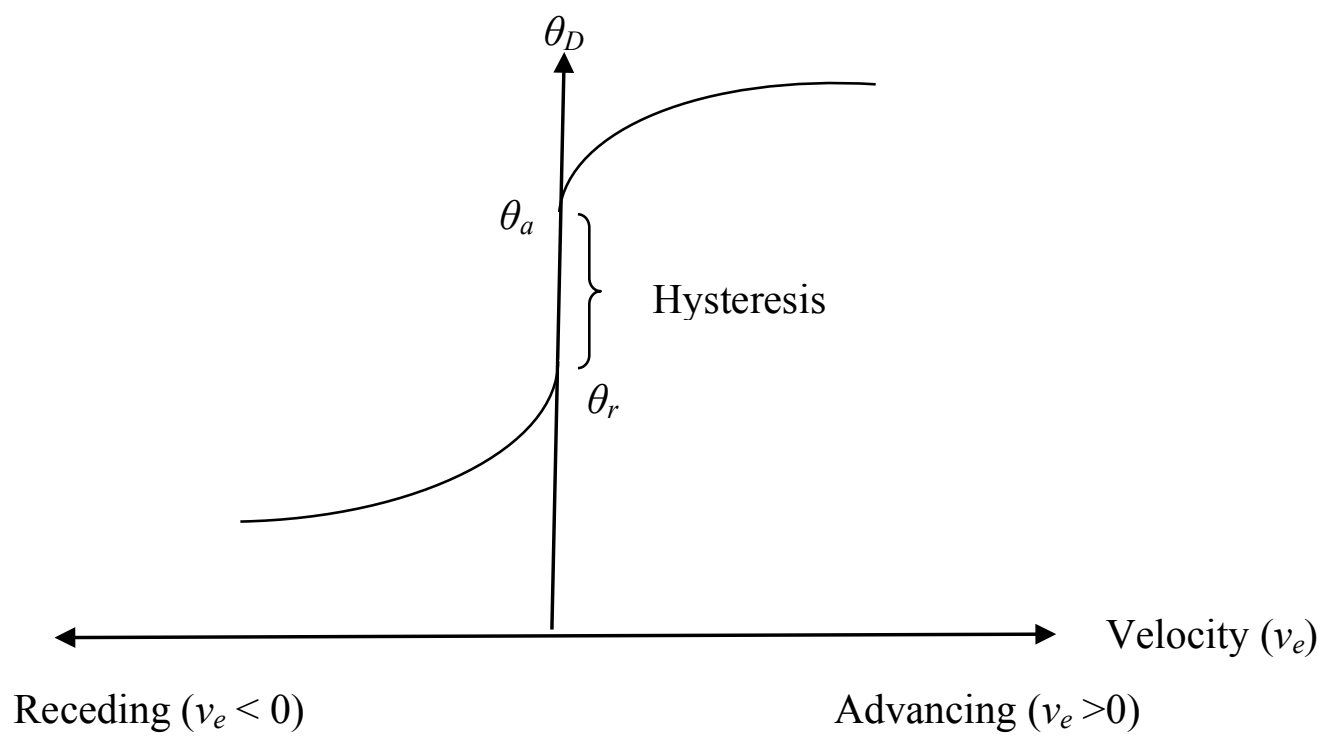

Figure 2.12: Dynamic contact angle $\left(\theta_{D}\right)$ variation with respect to contact line velocity $\left(v_{e}\right)$.

The dynamic contact angle commences when the interface is in relative motion and there after depends upon the rate of spreading. Wilkinson (1974) showed that the contact angle depends on the rate of motion of one of the interfaces. The experimental values of the dynamic contact angles depend on both velocity and the direction of the displacement (Blake, 1997). There are two main techniques to measure the dynamic contact angles, firstly the direct visualization (optical microscope) method, and secondly the capillary force (Wilhelmy plate) method.

\subsubsection{Kinetics of Wetting}

The dynamics of wetting can be quantified in terms of the relative velocity that the liquid moves across the solid (the contact-line velocity, $v_{e}$ ) and the dynamic contact angle $\left(\theta_{D}\right)$. This angle is the key boundary condition for the wetting process (Blake, 1997). Since surfaces are not ideally smooth and homogenous, it is often necessary to report the receding and advancing values rather than a single equilibrium contact angle. 
The spreading of a droplet of a liquid on a smooth solid surface can be described by the Hoffman-de Gennes law. It relates the edge speed, $v_{e}$, to the dynamic $\left(\theta_{D}\right)$ and equilibrium $\left(\theta_{e}\right)$ contact angles (McHale et al., 2009). That is,

$$
v_{e} \propto \theta_{D}\left(\theta_{D}^{2}-\theta_{e}^{2}\right)
$$

Throughout the last decade several researchers have developed models to relate the velocity of the contact line to the properties of the liquid, the interfacial properties, and the static contact angle (Bracke et al., 1989). When a drop of liquid is in contact with a solid, it will tend to spread spontaneously due to surface tension acting on it. As the shape of the drop changes with respect to time, the system dissipates some energy. Based on the effective energy dissipation modes for shape changes of spreading drops, different research groups have adopted one of three approaches to describe the spreading of a liquid on a solid surface (Ralston, 2008):

1. Hydrodynamics Model

2. Molecular Kinetics Model

3. Combined (Microhydrodynamic) Model

\subsubsection{Hydrodynamic Model of Kinetics of Wetting}

The hydrodynamic approach emphasizes the energy dissipation due to viscous flows in the core or wedge (Fig. 2.13) of the spreading droplet. In this approach, a relation between the capillary number $(\mathrm{Ca})$ and the value of the contact angle $(\theta)$ has been derived (Burley, 1976). Capillary number represents the ratio of the viscous to surface forces in dimensionless form. That is,

$$
C a=\frac{\mu v_{e}}{\gamma}
$$

where $\mu$ is the dynamic viscosity of the liquid. 
Complete wetting contact line dynamics can be studied using macroscopic (Ralston et al., 2008) or microscopic considerations. Under macroscopic considerations research groups explored the relationship between $v_{e}$ and $\theta_{D}$ in terms of the time variation of the base drop radius, $R(t)$. Tanner (1979) found a relationship between $\theta_{D}$ and $v_{e}$,

$$
\theta_{D}^{3} \propto v_{e}
$$

In simple terms, the change in the dynamic contact angle due to viscous bending of the gas/liquid interface can be written as,

$$
\theta_{D}^{3}-\theta_{m}^{3}=9 C a \ln \left(\frac{L}{L_{m}}\right) \quad\left(\text { For } \theta_{D}<3 \pi / 4\right)
$$

where $\theta_{m}$ is the microscopic angle $\left(\theta_{m}=\theta s\right), L$ is the macroscopic length scale and $L_{m}$ the microscopic length scale (Blake, 1997). It is noted this was not a physically acceptable equation due to the involvement of microscopic length scale.

In the complete wetting scenario, a microscopic precursor film may spread in front of the macroscopic edge (meniscus) due to van der Waals forces, as shown in Figure 2.13 (Voinov, 2006). This simplifies the hydrodynamic model by removing the involvement of the contact line.

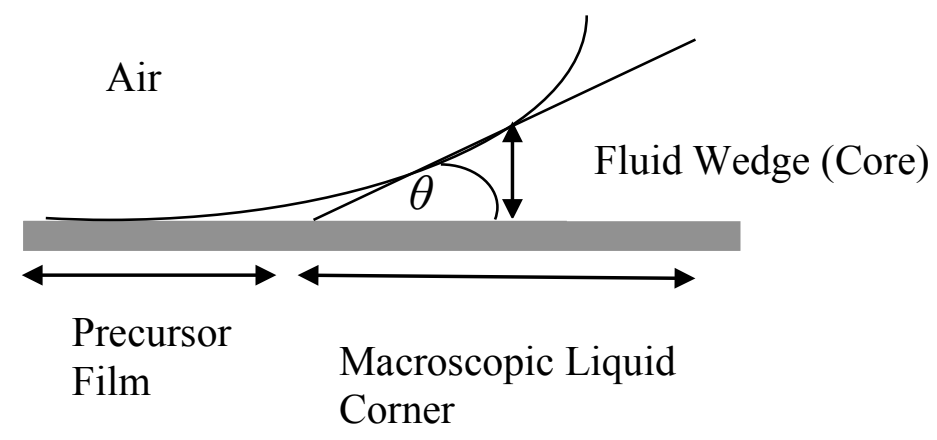

Figure 2.13: Spreading of microscopic precursor film in front of macroscopic edge (Butt et al., 2003).

For low contact angles and low contact line velocities, the hydrodynamic model gives the more appropriate picture (Wyart \& de Gennes, 1992). For the spreading of small 
drops with small contact angles, the kinetics of wetting mainly depends on the time, $t$, as per the hydrodynamic model. From Equation (2.26), scaling laws have been obtained for the drop's base radius or spreading rate $(R)$ and the dynamic contact angle, $\theta_{D}$.

$$
\begin{aligned}
& R(t) \sim t^{1 / 10} \\
& \theta_{D} \sim t^{3 / 10}
\end{aligned}
$$

These laws clearly show the drop's base radius and the dynamic contact angle both increase with time.

\subsubsection{Molecular Kinetics Theory of Wetting}

The Molecular Kinetic Theory (MKT) focuses on the dynamic three phase contact line with respect to the attachment or detachment of molecules of the fluid to or from the solid surface. According to this theory, the energy dissipation arises from the dynamic friction associated with the moving contact line (Frenkel \& Eyring, 1901).

Blake and Hayness (1969) pointed out that the dependence of the contact angle on velocity is controlled by the process of adsorption and desorption over the moving contact line.

The motion of the contact line is given by the statistical dynamics of the three-phase zone of molecules. The main parameters are the equilibrium frequency $\left(\mathrm{K}^{0}\right)$ of the random molecular displacements occurring within the three-phase zone and the average distance of each displacement, $\lambda$ (Bertrand et al., 2009) as shown in Figure 2.14. Hence, the wetting line velocity is predicted to be:

$$
v_{e}=2 K^{0} \lambda \sinh \left[\frac{\lambda^{2} \gamma}{2 k_{B} T}\left(\cos \theta_{S}-\cos \theta_{D}\right]\right.
$$

where $\theta_{S}$ is static contact angle, $k_{B}$ is the Boltzmann constant and $T$ the absolute temperature. 


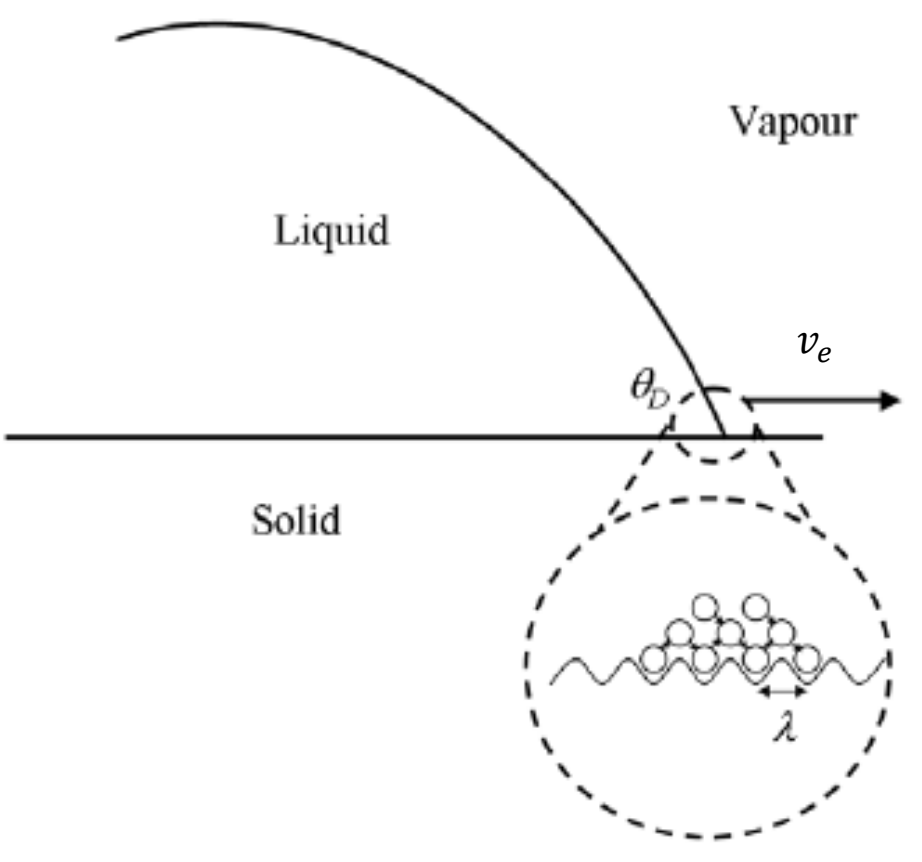

Figure 2.14: Parameters of moving contact line (Bertrand et al., 2009).

The MKT has been successful in predicting experimental results (Wyart \& de Gennes, 1992), especially when the contact angle and contact line velocity are high. However, it is still difficult to draw firm conclusions based on these theories due to a lack of understanding.

\subsubsection{Combined (Microhydrodynamic) Model}

It has been widely accepted that both types of dissipation exist simultaneously and numerous efforts have been made to develop a combined theory (Coninck et al., 2000). The problem lies in determining the relative importance of the disturbance of the microscopic contact angle due to the movement of the contact line and the modification of the meniscus profile by viscous flow in the liquid wedge region (Blake, 2006).

Petrov and Petrov (1992), as well as Brochard-Wyart and de Gennes (1992) have formulated integrated theories to address these issues. Brochard-Wyart and de Gennes explained the friction co-efficient $\zeta$ in terms of the molecular kinetics theory, and derived the following Equation (2.30). 


$$
v_{e}=\frac{1}{\zeta} \gamma\left(\cos \theta_{\mathrm{S}}-\cos \theta_{\mathrm{D}}\right)
$$

Blake and Clarke (1997) developed a simple hydrodynamic model for the macroscopic flow to predict the position of the contact (wetting) line based on the boundary layer theory. They used the molecular kinetic theory to explain the hydrodynamic stress between a liquid and a moving substrate.

Shikhmurzaev (1993) proposed a more accurate model from extended hydrodynamic concepts applied to a moving contact line. This model allowed for changes in surface properties within a small time scale. Shikhmurzaev used microscopic properties such as surface tensions, surface densities, surface viscosities and surface velocities for mass and force balances in this zone. Recent studies have confirmed that Shikhmurzaev's model fits the experimental results in the literature (Bertrand et al., 2009). Nevertheless the relationship between the molecular forces and the processes in the three-phase zone remain unresolved.

\subsection{Electrostatics}

\subsubsection{Introduction to Electrostatics}

Electrostatic forces were also used in this study to promote the collision and uptake of silica particles with water drops. Therefore, the main mechanisms of particle charging and other physical conditions related to electrostatic phenomena are reviewed.

Electrostatics explains how surface charges are exchanged during contact between different surfaces to produce static electricity. Electrostatic forces arise from the interaction between charged particles and electrostatic fields (Young, 1991). These forces are described by Coulomb's law (Section 2.2.2). When two objects are rubbed together it creates an equal amount of positive and negative charges. There are mainly two types of objects such as conductors and non-conductors (insulators). Charges can flow through conductors rapidly while non-conductors retain the charges. Charges can 
be measured using electrometers. The charge acquired by a particle depends on its size, dielectric constant and field intensity.

\subsubsection{Conductors and Non-conductors (Dielectric Particles /Insulators)}

When a conductor and a non-conductor are touched with a charged object at the same time, the conductor acquires the polarity, becoming charged very rapidly compared to a non-conductor. The conductivity of a particle depends on the free electrons, indeed the free flow of electricity. Most metals like copper, silver are good conductors while nonmetallic materials like glass, mica and rubber are good non-conductors (Young, 1991). Intermediate conductors may be initially polarized but mobility of these depends on their conductivity. If a good conductor particle and a good non-conductor (dielectric) particle are just separated from contact with a charged plate, the plate repels the conductor particle and the dielectric particle is neither repelled nor attracted by the plate.

Conductors have more free electrons to freely move causing flow of electric current. When a conductor acquires excess charge these are redistributed in a manner to reduce the total repulsive forces within the conductor. At this condition, the conductor is at electrostatic equilibrium and this directs the electric field of the conductor perpendicular to the surface. Non-conductors have strongly bound outer electrons, which make them resist electron flows.

\subsubsection{Mechanisms of Particle Charging}

There are 3 main techniques of charging particles.

1. Induction

2. Conduction

3. Tribocharging (Triboelectrification/Friction charging) 


\subsubsection{Induction}

Insulated neutral conductors can be charged by induction using a charged object. As shown in Figure 2.15, when a negatively charged rod is brought close to an uncharged conductor, the charges on the conductor separate. These separated charges can then be removed by a conductive path to the ground. When the conducting path is removed the conductor will remain with excess positive charge. This method of particle charging is called induction charging as it does not need a direct contact for charging. These inducted charges reside on the outer surface of the conductor. Similar induction occurs in the non-conductors.

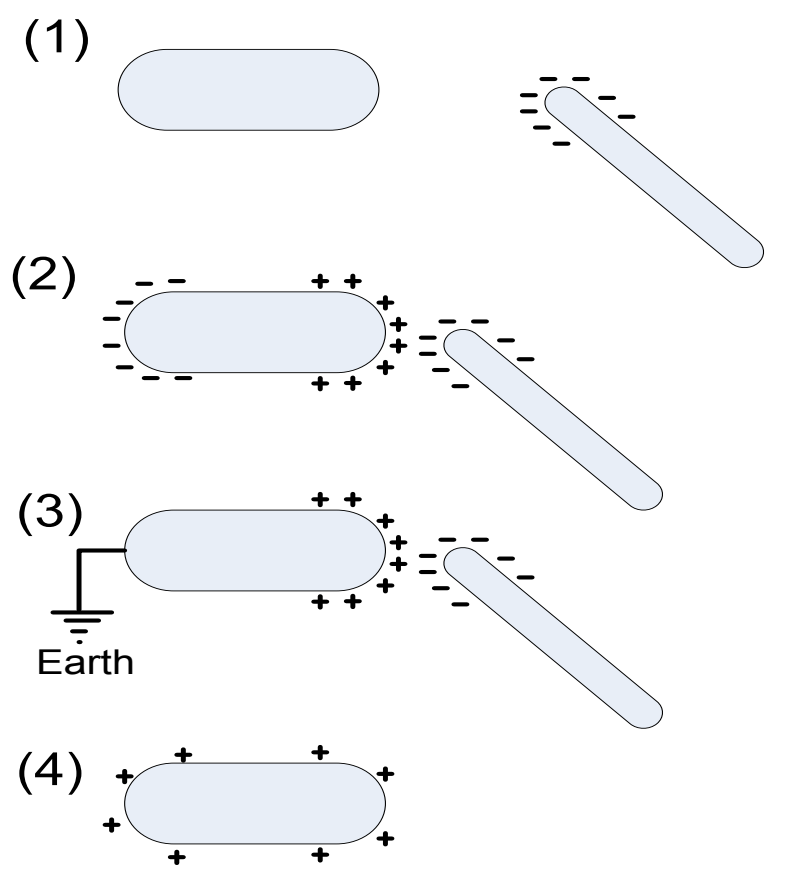

Figure 2.15: Charging by Induction (1) Charged rod brought close to an insulated conductor (2) Charge Separation (3) Earth Conductor (4) Remove charged rod.

\subsubsection{Conduction (Direct Contact)}

Neutral objects can also be charged by direct contact of a charged object as shown in Figure 2.16. When the charged object contacts the neutral object, excess charges in the charged object will move to the neutral object. After this flow comes to a equilibrium, both the object will have the same charge. Non-conductors cannot be charged by 
conduction. Unlike induction, the charged object loses some of its charge during conduction.

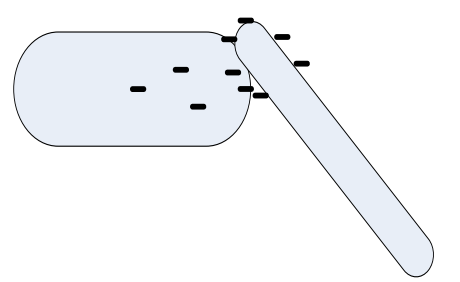

(1)

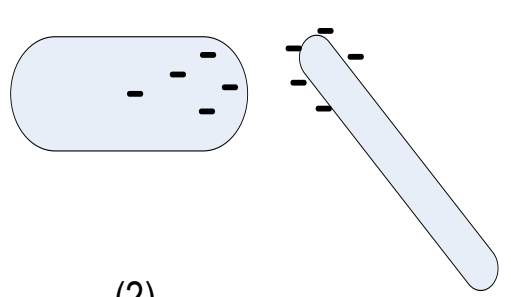

(2)

Figure 2.16: Insulated object charging by conduction.

\subsubsection{Tribocharging (Contact Charging/ Triboelectrification / Friction Charging)}

Tribocharging is the transfer of charge between two surfaces when they are rubbed together (Figure 2.17). Although this phenomenon was first recorded by the Greeks over 2000 years ago, it is still not well understood (Bailey, 2001). Tribocharging can occur between pairs of conductors, pairs of non-conductors and a conductor and a nonconductor.

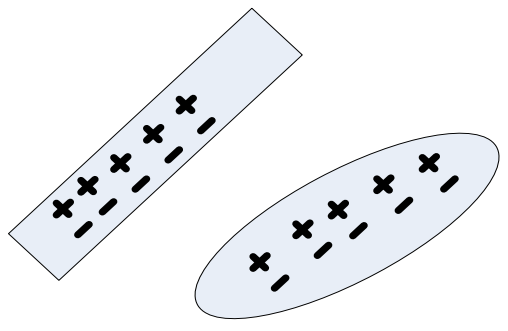

(a) Before rubbing

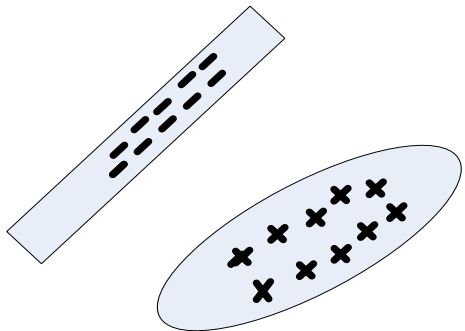

(b) After rubbing

Figure 2.17: Charging by Friction (a) Before rubbing (b) After rubbing

Tribocharging is controlled by the work function and the contact surface (Matsusaka, 2011). The work function is the energy required to remove an electron from its Fermi level (Lowell \& Rose-Innes, 1980; Gupta et al., 1992). The work function depends on both internal structure of the material and the surface condition such as presence of oxides and surface contamination (Gupta et al., 1992). Tribocharging of conductors (metals) can transfer charges until their Fermi levels equalize (Lowell \& Rose-Innes, 
1980). Charge transfer between a conductor and a non-conductor tends to transfer of charge until the work function difference between the two materials comes to a thermodynamic equilibrium.

Materials have been listed according to the magnitude and the polarity of charge acquired by friction with other materials (Lowell \& Rose-Innes, 1980; Lacks, D.J., 2012). This is known as the triboelectric series. Materials at the top of the series (such as glass, nylon...) obtain positive charge when they are contacted with the materials at the bottom of the series. Materials further apart on the table typically generate a higher charge than those that are closer together.

\subsubsection{Effects of Physical Conditions}

Conditions like room temperature, humidity, shape of particles, surface impurities, strain, and surface roughness of the particles have an effect on charging particles (Lowell \& Rose-Innes, 1980). For example room humidity affects the conductivity of air and thus the charging of particles. Note that a non-conducting particle may act like a conductor if it has adsorbed water at its surface due to atmospheric humidity. Hence experiments on non-conductors should be performed dry with a low humidity level.

If the particle surfaces are not properly cleaned then the impurities on the surface may give variations in conductivity. The electrostatic force experienced by an electrically charged fine particle is significantly higher than the gravitational force primarily because the surface area to mass ratio of a particle is inversely proportional to its diameter (Gupta, Gidaspow \&. Wasan, 1992). Also angular particles can discharge their charge more quickly than spherical objects because of the contact area and contact time (Ireland, 2012). 


\subsection{Summary}

The concept of selective collection relies upon the applied interfacial science, which is governed by surface forces, interfacial thermodynamics and kinetics of wetting. Surface forces such as van der Waals, electrostatic and hydrophobic forces operating across thin films control the particle-fluid interactions. The particle-drop adhesion involved in selective collection can be explained in terms of interfacial thermodynamics and kinetics of wetting.

To enable the adhesion of particles to water drops or bubbles there should be good wettability between the surfaces. The contact angle $\theta$ provides a direct measure of the wettability. The force balance between adhesive and cohesive forces determines the wettability or the degree of wetting. Young's equation predicts a value of the equilibrium contact angle, in terms of thermodynamic quantities such as the interfacial tension or surface energies at the three-phase contact line.

The contact angle defined by Young's equation is a static thermodynamic equilibrium value. If the three phase contact line is in motion, the angles produced are called dynamic contact angles. Also, real solid surfaces are not perfectly smooth and chemically homogeneous; hence there is no unique equilibrium contact angle. Hence in practice contact angle hysteresis is observed. The dynamics of wetting can be analysed in terms of dynamic contact angles.

Different hypotheses have been modelled to develop an understanding of the process of spreading of drops, and the associated change in the dynamic contact angles. The complete mechanism of wetting dynamics is not fully understood yet.

Electrostatics explains how surface charges are exchanged during contact between different surfaces to produce static electricity. Electrostatic forces arise from the interaction between charged particles and electrostatic fields. Conductors support the flow of electricity while non-conductors do not. Particles can be charged in electrostatics using three methods such as induction, conduction and tribocharging. 


\section{Chapter 3}

\section{APPLICATIONS INVOLVING PARTICLE-LIQUID-GAS INTERACTIONS}

\subsection{Introduction}

\subsection{Hydrodynamics}

\subsubsection{Forces Acting on Particles in Fluids}

3.2.1.1 Gravitational Force $\left(F_{G}\right)$

3.2.1.2 Buoyancy Force $\left(F_{B}\right)$

3.2.1.3 Drag Force $\left(F_{D}\right)$

3.2.2 Flow (Particle Drag) Regimes

\subsubsection{Terminal Settling Velocity $\left(v_{s}\right)$}

\subsubsection{Flow Fields}

\subsection{Particle Interactions through Liquid}

\subsubsection{Flotation Probabilities}

\subsubsection{Bubble-Particle Collision}

3.3.2.1 Probability of Collision and Bubble-Particle Collision Models

\subsubsection{Bubble-Particle Attachment}

\subsubsection{Probability of Attachment or Adhesion $\left(P_{a}\right)$}

\subsubsection{Bubble-Particle Detachment}


3.3.4.1 Probability of Detachment $\left(P_{d}\right)$

\subsubsection{Flotation Kinetics}

\subsubsection{Flotation Kinetics and Operating Variables}

3.3.6 Fine Particle Flotation

\subsection{Particle Interactions through Air}

\subsubsection{Wet Scrubbers}

\subsubsection{Granular Filtration}

3.4.3 Recent Research on Fine Particle Separation

\subsubsection{Liquid Marbles}

\subsubsection{Electrostatic Applications}
(a) Electrostatic Precipitation
(b) Electrostatic Coatings
(c) Electrostatic Fluidised Beds
(d) Electrostatic Separation

\subsection{Summary}




\subsection{Introduction}

This chapter focuses on the fluid-particle hydrodynamics and applications that involve particle-liquid-gas interactions, focussing on forces and different flow regimes. Particlegas interactions through a continuous liquid are discussed in Section 3.3. Froth flotation, which is a key example, involves a physicochemical separation process, which uses air bubbles to separate hydrophobic particles from its slurry. Froth flotation is used to separate complex or low grade ores when the average particle size is too small for efficient gravity separation or when the density difference between the minerals is too small. Despite much research, the understanding of flotation remains a challenging phenomenon. To understand the selective collection process it is essential to consider the earlier work on conventional froth flotation.

Particle-liquid interactions through a continuous gas phase are also important in many industrial applications. In particular, particle separations via the gas phase will be discussed. Relevant applications include particle removal devices such as wet scrubbers, granular filtration, particle separation techniques such as overflowing water troughs, and particle collection methods such as liquid marbles. Electrostatic charge is also a key parameter, controlling aerosol behaviour, for particle removal, separation, and powder coating.

\subsection{Hydrodynamics}

Hydrodynamics describe the dynamics of liquids in motion, including particle-liquid interactions, and applications. This section includes the forces acting on particles in fluids, and different flow regimes.

\subsubsection{Forces Acting on Particles in Fluids}

To model flotation processes, it is important to understand the forces acting on particles as they move in a fluid (gas or liquid). All particles moving in a fluid are subject to at 
least three forces: the gravitational force $\left(F_{G}\right)$, buoyancy force $\left(F_{B}\right)$ and hydrodynamic drag force $\left(F_{D}\right)$, as shown in Figure 3.1 (Seville et al, 1997).

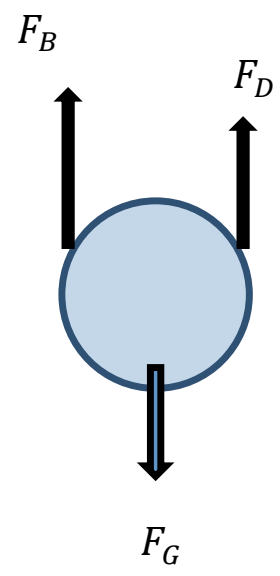

Figure 3.1: Forces acting on a downward moving particle in a fluid.

\subsubsection{Gravitational Force $\left(F_{G}\right)$}

The gravitational force is a body force that causes particles to settle. This force can be expressed as,

$$
F_{G}=m g=\rho_{p} V_{p} g
$$

where $m$ is the mass of the particle, $g$ the gravitational acceleration, $\rho_{p}$ the density of the particle and $V_{p}$ the volume of the particle.

\subsubsection{Buoyancy Force $\left(F_{B}\right)$}

The buoyancy force occurs due to the fluid displaced by the particles, and acts upward against the gravitational force. That is,

$$
F_{B}=m_{f} g=\rho_{f} V_{p} g
$$

where $m_{f}$ is the mass of the fluid displaced by the particle, and $\rho_{f}$ the fluid density. In gases, this force is often negligible compared to the gravitational force, given the low density of the gas. 


\subsubsection{Drag Forces $\left(F_{D}\right)$}

Drag forces occur due to the friction or shearing between a quiescent fluid and the particle and also due to the pressure difference between the front and back of the particle. The first category of the drag force is the friction or viscous drag while the second is the form or pressure drag. Both these drag forces depend on the fluid density and relative velocity between the particle and the fluid. The friction force always increases with an increase in the relative velocity, whereas the form drag may sometimes decrease with increases in relative velocity due to changes in the boundary layer structure, delaying the point of detachment.

The combined total drag force, $F_{D}$, may be expressed in terms of a drag coefficient $C_{D}$ (Seville et al, 1997):

$$
F_{D}=\frac{A \rho_{f} v^{2} C_{D}}{2}
$$

For spheres,

$$
F_{D}=\frac{\pi d_{p}^{2} \rho_{f} v^{2} C_{D}}{8}
$$

where $A$ is the cross- sectional area of the particle perpendicular to the direction of motion (for spheres $A=\pi d_{p}^{2} / 4$ ), and $v$ is the velocity of the particle relative to the fluid. The drag coefficient is a function of the particle shape, roughness, and the particle Reynolds number. That is,

$$
R e_{p}=\frac{d_{p} v \rho_{f}}{\mu_{f}}
$$

where $d_{p}$ is the diameter of the sphere and $\mu_{f}$ the viscosity of the fluid. The Figure 3.2 shows the variation of the drag co-efficient with the Reynolds number. 


\subsubsection{Flow (Particle Drag) Regimes}

Figure 3.2 provides the drag coefficient, $C_{D}$ as a function of the particle Reynolds number, $R e_{p}$. This curve consists of three main regimes (Flagan \& Seinfeld, 1988),

1. Laminar (Stokes') Region $-R e_{p}<1.92$

2. Intermediate (Transition) Region $-1.92<\mathrm{Re}_{\mathrm{p}}<508$

3. Newton's (Turbulent) Region $-508<\mathrm{Re}_{\mathrm{p}}<10^{5}$

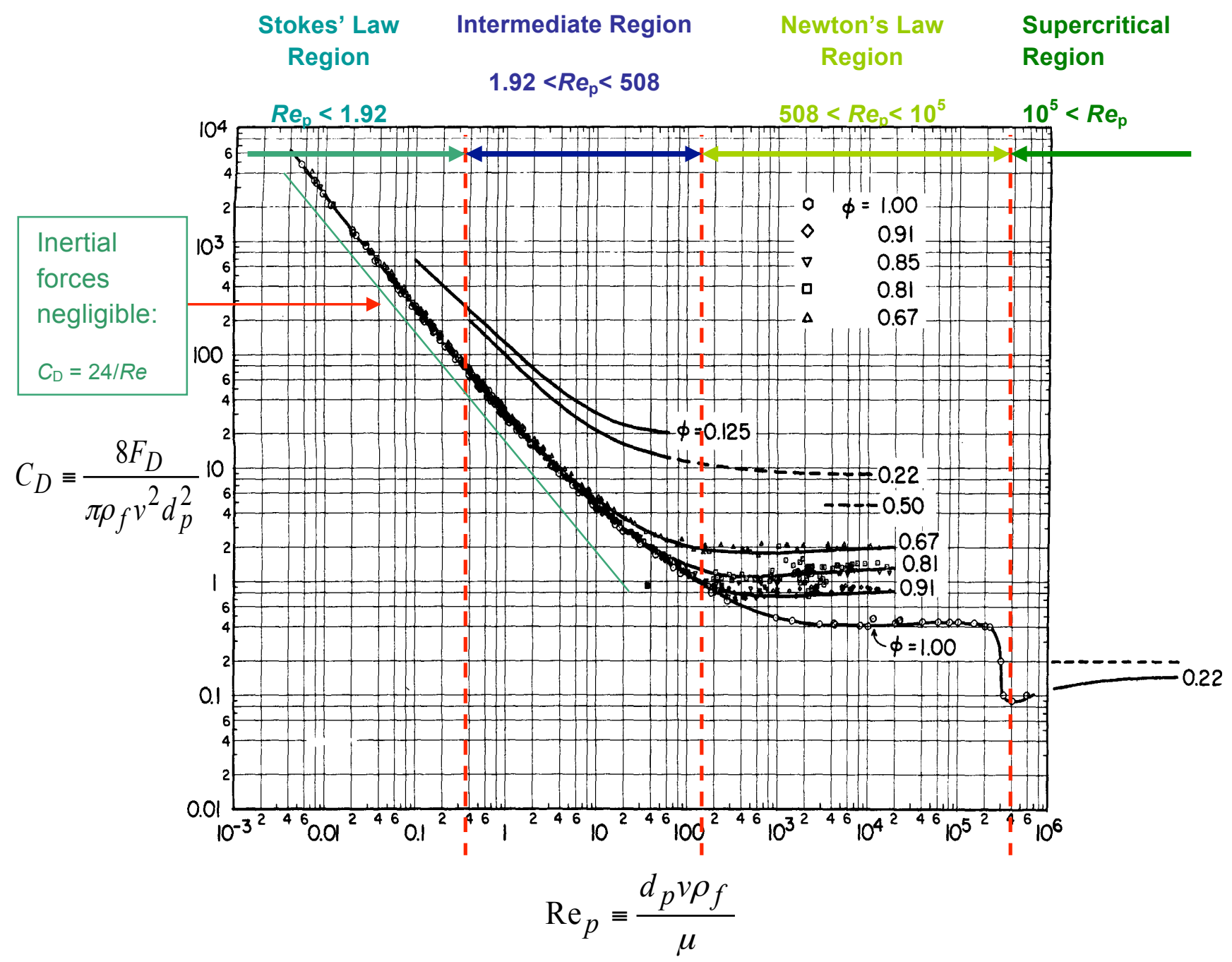

Figure 3.2: Drag Co-efficient as a function of Reynolds number for different shapes (Source: Haider and Levenspiel, 1989). Here $\Phi$ is the ratio between of the surface area of a particle having the same volume as a sphere to the surface area of the particle. 
There are different flow fields such as laminar (Stokes- $R e_{p}<1.92$ ), intermediate and turbulent (Newtons- $508<\mathrm{Re}_{\mathrm{p}}<10^{5}$ ) regions. In the laminar (Stokes) flow region the fluid moves in layers smoothly over the particle surface. Viscous forces dominate in this region, resulting in the drag coefficient,

$$
C_{D}=\frac{24}{R e_{p}}
$$

Hence the frictional drag force, $F_{D}$, acting on a spherical particle is,

$$
F_{D}=6 \pi \mu d_{p} v_{s}
$$

where $\mu$ is the dynamic viscosity $\left(\mathrm{N} \mathrm{s} / \mathrm{m}^{2}\right)$ and $v_{s}$ is the particle velocity relative to the fluid $(\mathrm{m} / \mathrm{s})$.

In the Newton's law region the flow around the particle is turbulent, and the inertial forces dominate over the viscous forces. In this region the drag coefficient, $C_{D}$ is approximately independent of the Reynolds number and equals 0.44 for spheres. In the intermediate region, $C_{D}$ can be expressed as (Flagan $\&$ Seinfeld ,1988);

$$
C_{D}=\frac{24}{R e_{p}}\left(1+0.15 R e_{p}^{0.687}\right)
$$

In general the drag force acting on a sphere is,

$$
F_{D}=C_{D} \frac{\pi}{8} \rho_{f} d_{p}^{2} v^{2}
$$

By equating the summation of the forces that act on a particle in a fluid, it is possible to obtain the terminal settling velocity.

\subsubsection{Terminal Settling Velocity $\left(v_{s}\right)$}

When a particle falls under gravity through a viscous fluid it will accelerate for a short while, but as the velocity increases, the drag force exerted by the fluid increases until the drag force is just equal to the net gravitational force less the buoyancy force. When these forces are in balance the particle does not accelerate any further. Hence the particle settles at a constant velocity. This velocity is called the terminal settling velocity, $v_{s}$ (King, 2002). 
The terminal settling velocity $\left(v_{s}\right)$ for a spherical particle can be evaluated by balancing the drag, weight and buoyancy forces. That is,

$$
v_{p}\left(\rho_{p}-\rho_{f}\right) g=\frac{C_{D}}{2} \rho_{f} v_{S}^{2} A
$$

Incorporating the Stokes' drag coefficient then gives,

$$
v_{s}=\frac{\left(\rho_{p}-\rho_{f}\right) d_{p}^{2} g}{18 \mu}
$$

where $v_{p}$ is the particle velocity, $\rho_{p}$ the particle density, $\rho_{f}$ the density of the fluid, $\mu$ the viscosity and $A$ the cross sectional area of the particle.

The above theory is only valid for solid spherical particles moving in quiescent fluids. If the fluid itself is turbulent, then particle-settling velocities are reduced. For instance, a particle may be small enough such that it settles in the Stokes regime, but if it finds itself entrained in a turbulent flow field, then it will not settle as quickly. Drops and bubbles have moving interfaces, and so can settle much more quickly. There are other effects that can complicate the settling of particles such as hindered settling, Brownian motion and non-continuum effects. Among these, Brownian motion and non-continuum effects are beyond the scope of this study.

Hindered settling applies when there are a large number of particles settling in a fluid (Seville et al, 1997). The settling velocity of particles in a suspension is much less than the terminal velocity of a single particle. In this situation Richardson and Zaki proposed the following correlation between hindered settling velocity $\left(v_{t}\right)$ and single particle terminal velocity $\left(v_{s}\right)$.

$$
v_{t}=v_{s} \varepsilon_{1}^{n}
$$

where $\varepsilon_{1}$ is the void fraction, i.e ;

$$
\boldsymbol{\varepsilon}_{1}=(1-\text { volume fraction of solids })
$$

The value of the Richardson-Zaki index, $n$, depends on the particle Reynolds number as shown in Table 3.1 . 
Table 3.1: Values for the Richardson-Zaki Index $(n)$

\begin{tabular}{|l|l|}
\hline$R e_{p}$ at Terminal Velocity & Value of $n$ \\
\hline$R e_{p}<10.2$ & 4.6 \\
\hline $0.2<R e_{p}<1$ & $4.4 \operatorname{Re}_{p}^{-0.03}$ \\
\hline $1<R e_{p}<500$ & $4.4 \operatorname{Re}_{p}^{-0.1}$ \\
\hline $500<R e_{p}$ & 2.4 \\
\hline
\end{tabular}

\subsubsection{Flow Fields}

Particles in a continuous fluid flow travel along the smooth continuous paths called streamlines, as shown in Figure 3.3. There are two different flow fields, known as laminar flow (Figure 3.3a) and turbulent flow (Figure 3.3b). The streamlines shown in Figure 3.3 are for fluid flow in a pipe. Laminar flow contains different flow streams, which slide on each other without mixing, while turbulent flow contains complex flows resulting from vigorous mixing of the fluid.

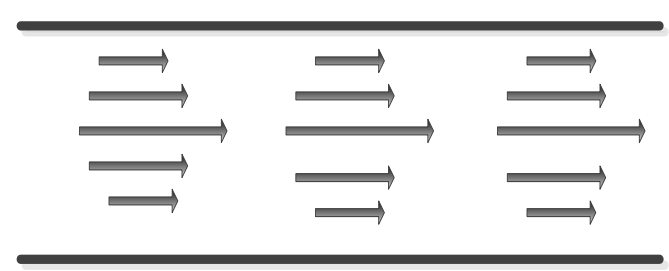

(a)

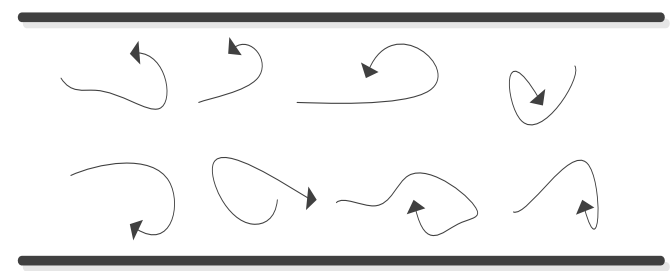

(b)

Figure 3.3: Streamlines of a pipe laminar flow and a pipe turbulent flow.

\subsection{Particle Interactions through Liquid}

In flotation, ore is finely ground and mixed with water to form a suspension. Air bubbles aerated through this suspension collide with the particles and carry away the hydrophobic particles. The mineral particles lifted by the air bubbles to the surface of the suspension form a froth layer, which is skimmed off. The separation in this process depends on the surface of the valuable mineral being hydrophobic and the gangue 
mineral being hydrophilic. The efficiency of the flotation process can be controlled by agitation and adding chemical reagents such as collectors, frothers, activators, depressants, $\mathrm{pH}$ and $\mathrm{E}_{\mathrm{h}}$ adjustments.

The core process of the recovery of the valuable minerals through flotation is the interaction between particles and air bubbles in water. Initial work on bubble-particle interactions was pioneered by Schuman (1942) and Sutherland (1948). Bubble-particle interactions have now been studied extensively by many investigators (Derjaguin \& Dukhin, 1961; Jameson et al., 1977, Ralston \& Dukhin, 1998; Yoon, 1999; Phan et al., 2003). There are three sub processes (micro-processes) that can occur (Jameson et al., 1977; Schulze, 1984) in the flotation process.

1. Bubble-Particle Collision (Encounter)

2. Bubble-Particle Attachment

3. Bubble-Particle Detachment

\subsubsection{Flotation Probabilities}

To enhance the overall flotation recovery it is desirable to increase the probability of collision and adhesion and reduce the probability of detachment.

The actual fraction of particles collected by the bubble is defined as the probability of collection. The probability $(P)$ or capture efficiency $(E)$ of a particle being collected by an air bubble during flotation has been described by Derjaguin and Dukhin (1960). That is,

$$
P=P_{c} P_{a} P_{s}
$$

The overall probability of collection $(P)$ depends on (Yoon \& Luttrell, 1985) the probability of collision or collision efficiency $\left(P_{c}\right.$ or $\left.E_{c}\right)$, probability of adhesion or attachment efficiency $\left(P_{a}\right.$ or $\left.E_{a}\right)$ and the probability of forming a particle-bubble aggregate $\left(P_{s}\right.$ or $\left.E_{s}\right)$. The term $P_{s}$ in the Equation (3.14) can be replaced by $\left(1-P_{d}\right)$, where $P_{d}$ is the probability of detachment,

$$
P=P_{c} P_{a}\left(1-P_{d}\right)
$$




\subsubsection{Bubble -Particle Collision}

Taggart (1927) was the first to focus on bubble-particle collision as an important step in the flotation process. Many other authors have now made major contributions on this topic (e.g. Sutherland \& Wark, 1955; Gaudin, 1957; Kitchener, 1972; Jameson, 1977; Dukhin \& Ralston, 1998; Nguyen \& Schulze, 2004; Shahbazi et al, 2010). The capture of the particle by a bubble can be analysed as a sequence of stages. First the particles and bubbles need to come into close proximity (Jameson et al., 1977). When the distance between the bubble and the particle is small, the liquid between them drains to leave a film. For the collision of the particle and the bubble to be successful in rupturing this film, it is essential to reduce their relative velocity by having a high viscous stress on the thin film.

Derjaguin and Dukhin (1961) suggested a three-zone model for the collision mechanism of small and medium size particles considering surface forces as shown in Figure 3.4. This model was the first attempt to describe the three zones that a particle must pass through before it adheres to the surface of an air bubble.

Zone 1 is the outer hydrodynamic zone where the particle is acted on by only hydrodynamic drag and gravity. The hydrodynamic drag force tends to sweep the particles around the bubble. Zone 2 is the diffusion-phoretic (diffusiopheretic) zone where the diffusional force, electropherotic force, and surface force, also become active. The surface forces or molecular forces acting in Zone 3 (the wetting zone), determine the behaviour of the thin film between the bubble and the particle. If the particle is hydrophobic the surface forces give rise to an attractive disjoining pressure, which results in particle-bubble attachment. 


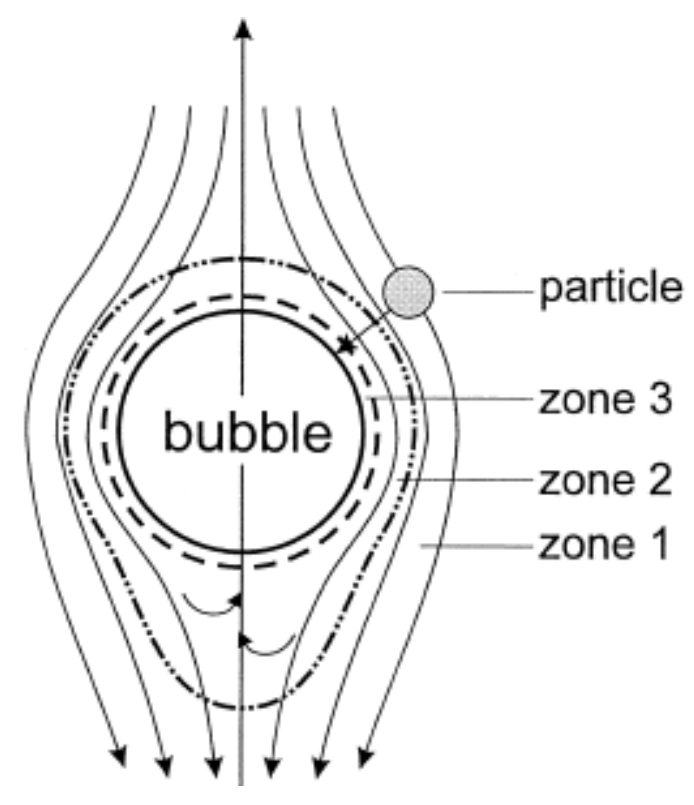

Figure 3.4: Three zones around the particle in flotation process: Zone 1 . Hydrodynamic; Zone 2. Diffusiopheretic ; Zone 3. Surface Force Zone (Ralston et al., 1998).

When a particle reaches close to the bubble surface, high viscous stress on the thin film causes drainage of the thin liquid film between the two surfaces. This is also called Taylor drainage. Due to this drainage, the particle experiences a hydrodynamic resistance, which reduces the normal component of the particle's velocity towards the bubble surface as shown in Figure 3.6. The particle is pushed towards the bubble surface by this velocity difference. This inertial pushing force is called the hydrodynamic pressing force $\left(F_{p}\right)$ (Derjaguin \& Dukhin, 1984). The centrifugal force, $F_{c}$, which prevents particle deposition near the equator originate from the tangential component of the particle velocity. This centrifugal force is referred to as the inertial negative force while the inertial pushing force is referred to as the inertial positive force. 


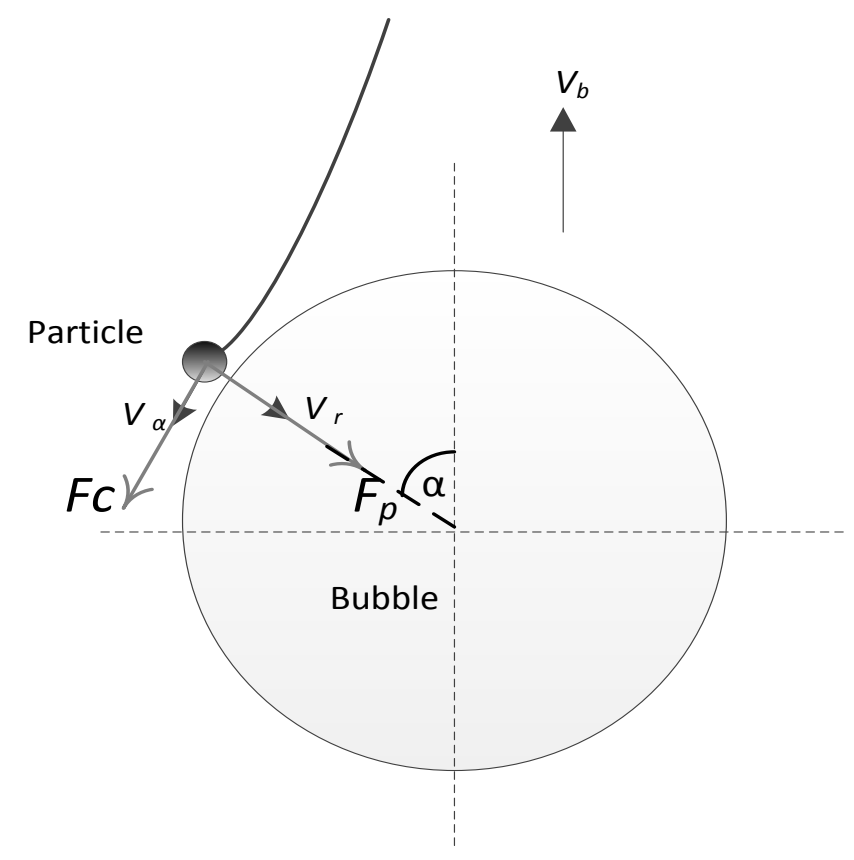

Figure 3.5: Velocity components $\left(\mathrm{V}_{\mathrm{r}}\right.$, radial velocity and $\mathrm{V}_{\alpha}$, tangential velocity) of the particle approaching a bubble travelling with an upward velocity of $\mathrm{V}_{\mathrm{b}}$.

Models attempt to combine the effect of each of these parameters to predict the overall bubble-particle collision efficiency.

\subsubsection{Probability of Collision and Bubble- Particle Collision Models}

Many bubble-particle collision models have been developed in the flotation literature to predict the collision efficiency, $P_{c}$ (Langmuir-Blodgett, 1945; Sutherland, 1948; Gaudin, 1957; Webber \& Paddock, 1983; Yoon \& Luttrell, 1989; Schulze, 1989; Nguyen-Van, 1992; Dukhin \& Ralston, 1998). Numerous variables affect the probability of collision $\left(P_{c}\right)$ such as particle and bubble sizes, particle charge, difference between density of the particle and density of the suspension, number concentration of bubbles in the suspension, directional angle of impact, velocity of collision, bubble surface mobility, concentration of reagents, $\mathrm{pH}$ and the intensity of agitation.

There are four bubble-particle collision mechanisms such as inertia, gravity, interception and Brownian diffusion. Denser and coarser particles have a high probability of collision due to their higher inertia. Hence, these particles are unable to 
follow the fluid stream lines around the bubbles and continue on their path towards the bubble. Brownian diffusion applies only for very fine particles, which are less than 1 $\mu \mathrm{m}$ (Miettinen et al., 2010) and will not be discussed further.

Langmuir and Blodgett (1945) developed the first collision model (Eq. 3.16) for the inertial-hydrodyanmic interaction between a small stationary droplet and a large falling droplet.

$$
P_{C-L B}=\left(\frac{K}{K+0.2}\right)^{2}
$$

where $K$ is the Stokes number based on a particle's inertial forces and fluid viscous resistance. That is,

$$
K=\frac{\rho_{p} v_{b} d_{p}^{2}}{9 \mu d_{b}}
$$

Derjaguin and Dukhin (1961) showed that the hydrodynamic fields around a rising bubble and a falling droplet are identical. Their inertial deposition model is applicable to particle-bubble collisions involving particles with large Stokes numbers.

Sutherland (1948) derived an expression for particle bubble collision from fluid stream functions. The probability of collision can be defined as the fraction of the particles in the path (tube) of a rising bubble (Yoon and Luttrell, 1986). Sutherland noted that a stream of collision radius (grazing radius) $R$ as shown in Figure 3.5 can be defined; such that all particles within the tube will, in principle, collide. 


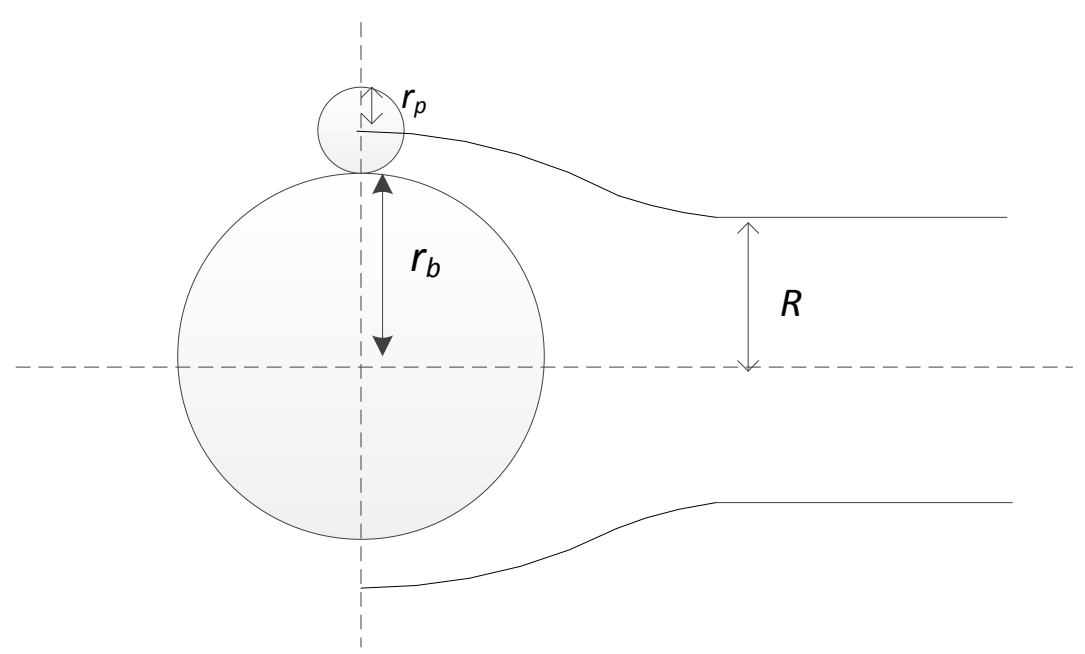

Figure 3.6: Trajectories of particles on streamlines.

The collision efficiency $\left(P_{c}\right)$ is (Sutherland, 1948):

$$
P_{c-S U}=\frac{3 r_{p}}{r_{b}}
$$

where $r_{p}$ is the radius of the particle and $r_{b}$ the radius of the bubble. All particles in the stream tube ahead of the bubble area have the potential to be captured. Fundamentally the above collision efficiency depends on the solution to the equations of motion of the two bodies (Ahmed \& Jameson, 1989). Most of the collision models have been developed based on this basic concept (Nguyen et al., 2003).

Flint and Howarth (1971), and Reay and Ratcliff (1975) have also obtained theoretical relationships for the particle-bubble collision efficiency using the Stokes' stream function. They have showed the collision efficiency $\left(P_{c-F R}\right)$ for the case of fine particles $\left(d_{p}<20 \mu \mathrm{m}\right)$ and very small bubbles $\left(\mathrm{d}_{\mathrm{b}}<100 \mu \mathrm{m}\right)$ is,

$$
P_{C-F R} \alpha\left(\frac{d_{p}}{d_{b}}\right)^{N}
$$

where $N$ is approximately 2, neglecting the inertia of the particles (Ahmed \& Jameson, 1989; Dai et. al, 2000). 
Yoon and Luttrell derived another model based on similar assumptions to the Sutherland model. They assumed the inertialess particles collide with the bubble only over the upper half of the bubble surface. Also this model considered interception and an empirical stream function (Yoon and Luttrell, 1989). This is given in the following equation;

$$
P_{C-Y L}=\left(\frac{3}{2}+\frac{4 R e_{b}^{0.72}}{15}\right)\left(\frac{d_{p}}{d_{b}}\right)^{2}
$$

where $R e_{b}$ is the Reynolds number of the bubble. Yoon and Luttrell have also considered different flow conditions in the fluid by incorporating the Reynolds number. Schulz (1989) developed a collision model incorporating the influence of the particle inertial force, although he neglected the negative effect of the inertial forces.

Dukhin (1998) proposed a more detailed and complete model (Eq.3.21) for bubbleparticle collision which is known as the Generalised Sutherland Model (GSE model).

$$
P_{c-G S E}=P_{c-S U} \cdot \sin ^{2} \theta_{t} \cdot \exp \left\{3 K_{3}\left[\cos \theta_{t}\left(\ln \frac{3}{P_{c-S U}}-1.8\right)-\frac{2+\cos ^{3} \theta_{t}-3 \cos \theta_{t}}{2 P_{c-S U} \sin ^{2} \theta_{t}}\right]\right\}
$$

where $K_{3}=K \cdot \frac{\rho_{p}-\rho_{f}}{\rho_{p}}=\frac{2 v_{b}\left(\rho_{p}-\rho_{f}\right) r_{p}^{2}}{9 \mu r_{b}}$ and $\theta_{t}$ is the angle of tangency.

This model provided reliable agreement between the experimental and calculated collision efficiencies for mobile bubble surfaces and intermediate Stokes numbers considering both the positive and negative effects of inertia on the collision process. For example when the particle inertia is neglected this model overestimates the collision efficiency.

Most collision models for conventional flotation have used the Stokes' law of fluid resistance. Experimental measurements of the bubble-particle interaction force have proved that at low approaching speeds the surface forces dominate while at high velocities the hydrodynamic drag force becomes prominent (Nguyen, 2003). The forces that affect the motion of particles include gravitational, inertial (due to rotation of mass) and hydrodynamic drag forces. Schulze (1989) introduced a model incorporated these interception, gravitational and inertial effects. 
However, none of these models can predict the bubble-particle collision efficiency of a flotation cell correctly (Ralston, 2000). Many of them suffer from unrealistic assumptions like uniform distribution of collision over the entire upper half surface of the bubble; the bubble surface is completely immobilised (retarded) by the adsorption of surface active materials, the movement of both particles and fluid near the bubble surface is retarded and no inertial force influence for the collision. All these collision models predict that the collision efficiency increases with particle size.

\subsubsection{Bubble - Particle Attachment or Adhesion}

The attachment of a hydrophobic particle to an air bubble is a critical step in the flotation process. Numerous attempts have been made to study the micro-process of particle-bubble attachment (Sutherland, 1948; Ye \& Miller, 1988; Ahmed \& Jameson, 1989; Aplan F.F., 2001; Miettinen et al., 2010). These studies have considered the bubble-particle attachment in terms of attachment (induction), contact (sliding) times and surface forces.

Solid-gas bubble attachment comprises 4 steps as illustrated in Figure 3.7 (Aplan F.F., 2001). These are:

1. Thinning of the liquid film between the bubble and the particle to the critical thickness

2. Rupture of the liquid film and formation of a three phase contact

3. Recession of the liquid film, whereby liquid is replaced by air to form a stable contact attaching the bubble to the hydrophobic surface

The total bubble-particle attachment time or induction time $\left(t_{a t}\right.$ or $\left.t_{\text {ind }}\right)$ is given by the summation of the time taken for these three steps. Experimental results have proven that the $t_{a t}$ increases with the particle size and decreases with the surface hydrophobicity (Ye and Miller, 1988). 


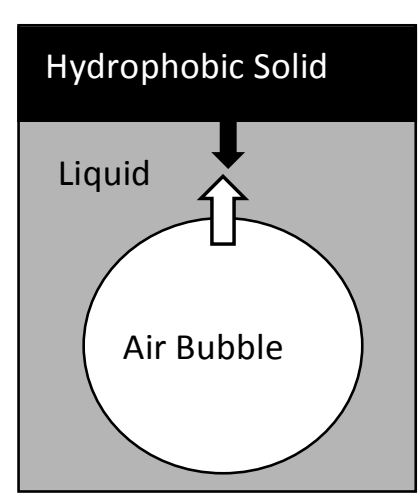

(a)

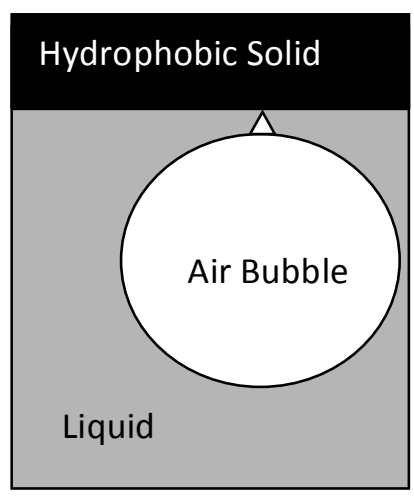

(b)

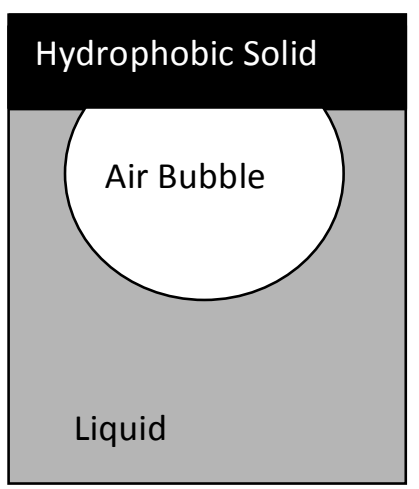

(c)

Figure 3.7: Stages of forming bubble-particle aggregate: (a) Film thinning (b) Film rupture.

There are two main attachment models, those of Dobby and Finch (1987) and Yoon and Luttrell (1994). Both of these are based on the magnitude of the attachment time ( $t_{a t}$ or $\left.t_{\text {ind }}\right)$ and contact time $\left(t_{\text {contact }}\right.$ or $\left.t_{s}\right)$ (Ralston et al., 1998).

During bubble-particle collision the bubble surface starts to deform. Depending on the behaviour of a particle that reaches a bubble's surface two scenarios can be formed. In the first scenario, the particle will rebound without sliding along the surface of the bubble. Under this condition, the contact time $\left(t_{\text {contact }}\right.$ or $\left.t_{s}\right)$ is equal to the time taken for the impact between the particle and the bubble. In the second scenario the particle will slide along the surface of the bubble. In this case, the contact time will be determined by the collision angle $(\varphi)$ and it is equal to the sliding time (Ralston et al., 1998). The collision angle is the angle between the vertical line and the point at which the particle contacts the surface of the bubble $(\varphi<\pi / 2)$ as shown in Figure 3.8. 


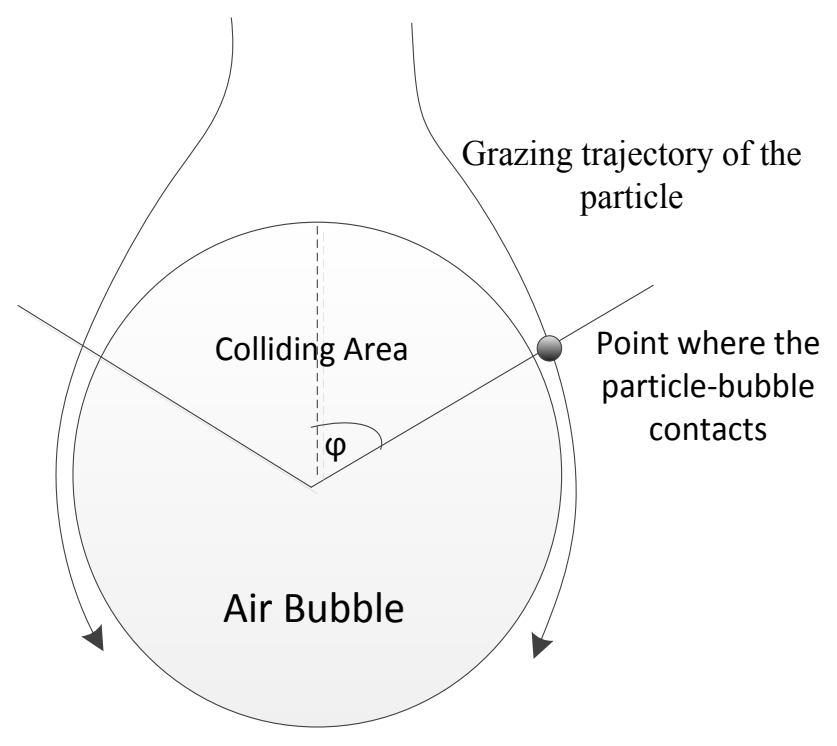

Figure 3.8: Collision angle and the colliding area of the bubble-particle collision.

When the particle approaches the bubble via a straight line, the collision deforms the bubble. In this case the contact time is called the collision time $\left(t_{c}\right)$. The collision time, $t_{c}$, is estimated to be the half period of the bubble deformation. The collision time is usually shorter than the sliding time, $t_{s}$ (Nguyen et al., 2003) for the attachment to occur. Also the attachment time ( $t_{a t}$ or $\left.t_{\text {ind }}\right)$ must be smaller than the contact time $\left(t_{\text {contact }}\right)$ (Sutherland, 1948). However, the contact time hypothesis is not favourable for very fine particles (Ahmed \& Jameson, 1989). The available attachment models are far from satisfactory (Nguyen et al., 2003). It is still very difficult to predict the actual attachment time.

The bubble-particle attachment results from the sequential occurrence of these three steps (Nguyen \& Evans, 2004). The hydrophobic force is the principal parameter controlling the bubble-particle interaction, leading to the particle attachment. Hydration forces and steric forces are the other surface forces that may become significant under specific conditions, although the water film rupture occurs at distances larger than the range of these forces. 


\section{Thinning of the liquid film}

Thin film phenomena determine the thinning of the water film between the particle and the drop. Derjaguin and Kasakov (1939) first investigated this. Spherical bubbles can be pressed easily by the solid particle due to external pressure and capillary forces. As the particle and the bubble approach each other, the hydrodynamic resistance force increases due to the small separation distance between these two bodies. The viscous stress created by the hydrodynamic flow of the liquid film between the two bodies can either thin and rupture the intervening film or bounce back the particle (Jameson, 1977; Leja, 2004). When the particle and air bubble become closer than $1000 \mathrm{~nm}$ or less, the liquid between them forms a thin film (disjoining film) as the molecular forces come in to play. A disjoining pressure, which originates on this thin film then determines the rupture of the film (Paulsen et al., 1995).

The disjoining pressure, $\pi$, represents the excess pressure between the bubble $\left(P_{b}\right)$ and the bulk liquid $\left(P_{l}\right)$ adjacent to the solid surface. The disjoining pressure mainly consists of three components, van der Waals force $\left(\pi_{d}\right)$, electrostatic component $\left(\pi_{e}\right)$ and structural component due to a hydration layer, where the structure of water dipole differs from the completely random bulk structure $\left(\pi_{s}\right)$. The disjoining pressure between a bubble and a hydrophobic particle is attractive making the liquid film unstable leading to its rupture. This attaches the bubble to the hydrophobic particle forming a contact between two surfaces. The disjoining pressure that exists in the film between a bubble and a hydrophilic particle is repulsive, preventing bubble-particle attachment (Nguyen $\&$ Schulze, 2004).

\section{Rupture of the liquid film and formation of three-phase contact}

Different theories have been proposed to explain the liquid film rupturing (Nguyen et al, 1997; Jaczuk, 2006). Generally it is believed that when the hydrodynamic flow and the initial capillary thinning can bring the two bodies within the range of negative disjoining pressure, this results in film rupture. This can also result from the rapid instability due to density fluctuations. The minimum distance required for the bubbleparticle attachment depends on the relative momentum of the two bodies. The high viscosity of water is one of the main barriers to the bubble-particle attachment. Thus 
there are benefits in using a drop-particle system, rather than a bubble-particle system, due to the low viscosity of the surrounding air film.

\section{Recession of the film and formation of aggregate}

The last stage of the particle attachment is the film recession stage where the point of rupture forms a stable contact angle with the particle. This contact angle is formed as a result of the formation of the three phase contact (TPC) line as discussed in Chapter 2. Once the stable contact angle is formed, capillary forces will govern the strength of adhesion (Ralston et al., 1998). It was proven experimentally that the surface forces have little effect on the particle motion until the film ruptures (Nguyen \& Evans, 2004).

\subsubsection{Probability of Attachment or Adhesion $\left(P_{a}\right)$}

Probability of adhesion depends on the value of the contact angle, angle of impact, velocity of collision, viscosity, and contact time. The probability of adhesion can be defined as the fraction of particles whose contact times are longer than the attachment time (Luttrell \& Yoon, 1992). This probability also can be predicted by considering the kinetic energy of the particles (Yoon \& Mao, 1996). The attachment kinetic energy of the particles $\left(E_{k}\right)$ should be larger than the energy barrier $(E)$ for attachment to occur. This energy barrier, $E$, can be determined by the surface forces such as van der Waals force, electrostatic force and hydrophobic force involved in the bubble-particle interactions while $E_{k}$ can be calculated from the tangential velocities of the particle.

$$
P_{a-Y M}=\exp \left(-\frac{E}{E_{k}}\right)
$$

Dobby and Finch (1987) proposed that the attachment efficiency depends on the maximum collision angle $\varphi_{\mathrm{c}}$.

$$
P_{a-D F}=\frac{\sin ^{2} \varphi_{a}}{\sin ^{2} \varphi_{c}}
$$

where $\varphi_{a}$ is the adhesion angle or any collision angle. 
Yoon (1999) derived another expression for the probability of particle-bubble attachment based on the contact time. This equation is valid for the intermediate Reynolds number range.

$$
P_{a-Y=\operatorname{Sin}^{2}}\left[2 \tan ^{-1} \exp \left(\frac{-\left(45+8 R e^{0.72} v_{b} t_{\text {ind }}\right.}{15 d_{b}\left(\frac{d_{b}}{d_{p}}+1\right)}\right)\right]
$$

As per this equation attachment efficiency depends on bubble velocity $\left(v_{b}\right)$, flow around the bubble, contact angle, and both the bubble and particle diameters.

\subsubsection{Bubble - Particle Detachment}

External forces may cause the attached particle to detach from the bubble. Hence, quantification of particle-bubble aggregate stability is important. This detachment occurs if the adhesive force is insufficient to resist the dynamic forces acting on the particle in a flotation cell (Nguyen et al., 2003).

Figure 3.9 is a schematic representation of a small particle suspended beneath a large gas bubble. The particle experiences adhesive forces due to the capillary force $\left(F_{c}\right)$ acting around the three-phase contact line, the gas pressure force acting on the area inside the three phase contact line $\left(F_{h}\right)$ and the buoyancy force of the liquid acting on the submerged particle surface $\left(F_{b}\right)$. The hydrostatic pressure of the liquid column with height $\mathrm{H}$ acts against the adhesive forces. Fluid drag forces can also arise. A critical detachment force exists (Nguyen et al., 2003), dependent on the maximum strength of the particle meniscus, which depends on the particle and the bubble size, the liquid density, the surface tension and the contact angle. 


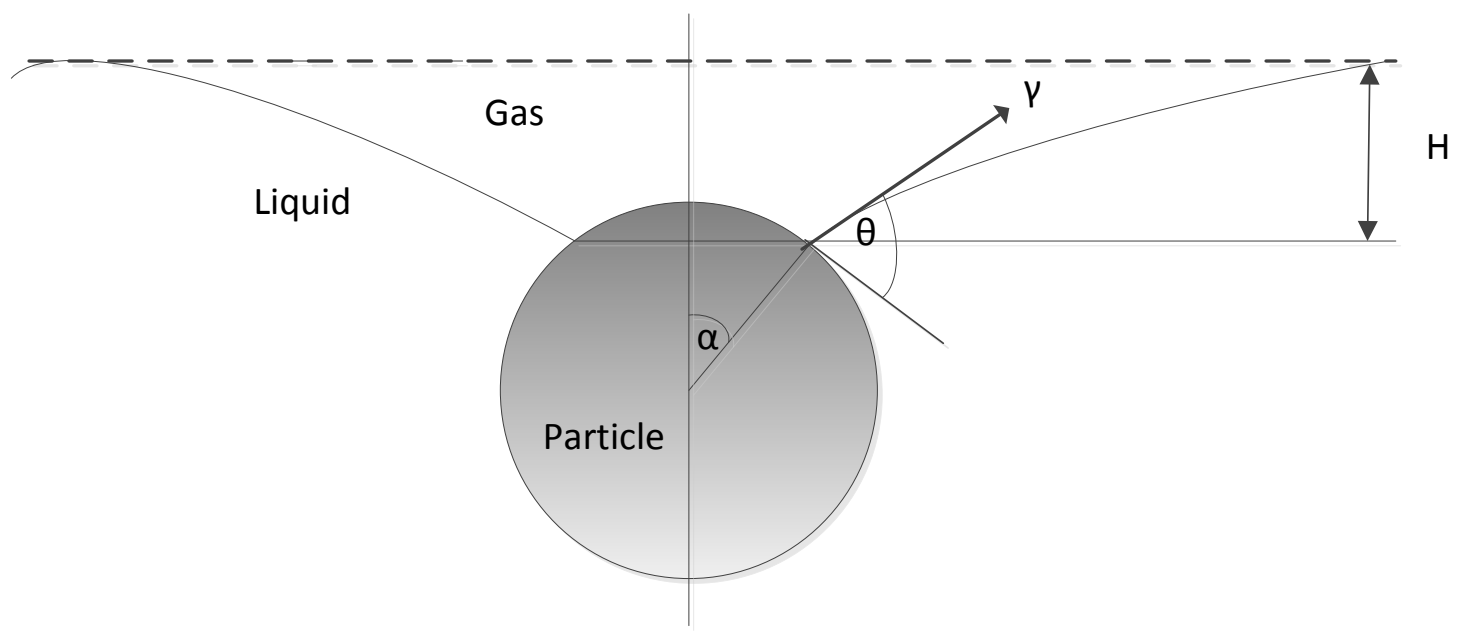

Figure 3.9: Particle attached to a larger air bubble (Nguyen et al., 2004).

Particle detachment is caused by a combination of the particle weight $\left(F_{g}\right)$ and turbulent accelerating inertial forces $\left(F_{t}\right)$. Detachment takes place only when the detaching force exceeds the maximum strength of the particle-meniscus or the tenacity.

A number of studies have been undertaken on bubble-particle attachment, while relatively few have been concerned with particle detachment forces ( Gaudin, 1957; Mika \& Fuerstenaun, 1968 and Schulz,1977). By analysing the particle detachment forces we can predict the maximum floatable particle size (Ahmed \& Jameson, 1989). However, turbulent eddies in a gas have much less energy, so once successful collision has taken place, then there is a low probability of detachment.

\subsubsection{Probability of Detachment $\left(P_{d}\right)$}

Particle-bubble stability depends on the strength of the bond and the hydrodynamic conditions in the agitated suspension. The probability of detachment can be defined as a function of the work of adhesion $\left(W_{a}\right)$, the energy barrier $(E)$ and the kinetic energy of the particle $\left(E_{k}\right)$ in a given particle trajectory (Yoon \& Mao, 1996).

$$
P_{d}=\exp \left(-\frac{W_{a}+E}{E_{k}}\right)
$$

As discussed in chapter 2,

$$
W_{a}=\pi r_{p}^{2} \gamma(1-\cos \theta)
$$


The probability of detachment also depends on a few other factors such as residence time, gravitation, density and hydrophobic properties. There may be multiple events over the life of the aggregate.

The above review is limited to the major factors relevant to the present thesis. In general, flotation recovery also depends on the flotation hydrodynamics (air hold up, bubble surface mobility, suspension densities, residence time, and mixing), and chemical properties of the frother and collector reagents. These factors are largely irrelevant to the present thesis and are therefore not reviewed.

\subsubsection{Flotation Kinetics}

Flotation kinetics is mainly determined by the surface force parameters and hydrodynamic parameters. These parameters include the chemistry of the mineral-water interface, particle bubble collision efficiency, particle size and the strength of attachment of particles to the bubbles. From a chemical kinetics analogy, the kinetics of the flotation is often modelled by the ordinary differential equation,

$$
\frac{d C_{p}}{d t}=-k C_{p}^{n}
$$

where $C_{p}$ represents the concentration of floatable particles, $t$ is the flotation time, $k$ is the flotation rate constant and $n$ is the order of flotation. Here concentration $(C)$ can be defined as the mass of floatable particles per unit volume $(C=m / v)$.

\subsubsection{Flotation Kinetics and Operating Variables}

In this section a number of factors governing flotation kinetics are examined, covering the effects of the particle size, bubble size, bubble coalescence and contact angle.

\section{(a). The Effect of Particle Size on Flotation Rate}

Figure 3.10 shows the flotation recovery for different sulphide particle sizes. 


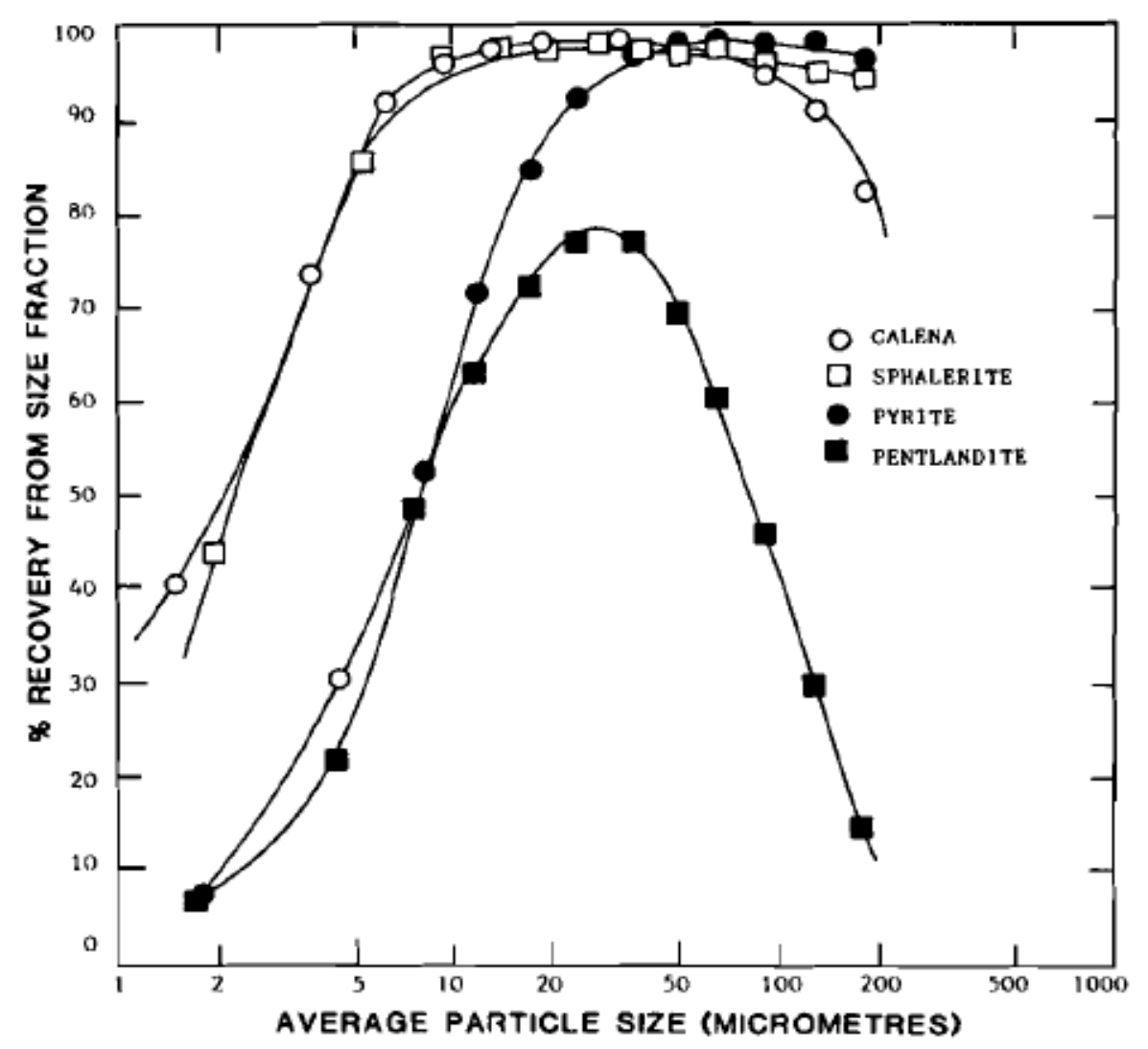

Figure 3.10 : Recovery of different size of sulfide minerals after 60 seconds flotation batch tests (Source :Jowett, 1980).

As shown in Figure 3.10 mineral flotation is most successful in the intermediate size range of 10-100 $\mu \mathrm{m}$ (Nguyen \& Schulze, 2004). The flotation recovery is low for both fine $(<10 \mu \mathrm{m})$ and coarse particles $(>100 \mu \mathrm{m})$. The optimum floatable size range also depend on mineral physical properties such as density and shape. Fine particles may result in poor recovery due to the low collision probability with air bubbles and entrainment in the froth. Low flotation recovery of fine particles will be discussed in Section 3.3.6.

Coarser particles may result in poor recovery due their greater mass which leads to lower floatability with air bubbles (Jameson et al, 1977). This is consistent with Sutherland's equation in the above section. It is noted that $P_{d}$ increases with increasing particle size, but can be neglected for small $(<20 \mu \mathrm{m})$ particles. 
Figure 3.11 shows the strong dependence of the rate constant on the particle-size and the bubble diameter (Jameson et al., 1977; Ahmed \& Jameson, 1989).

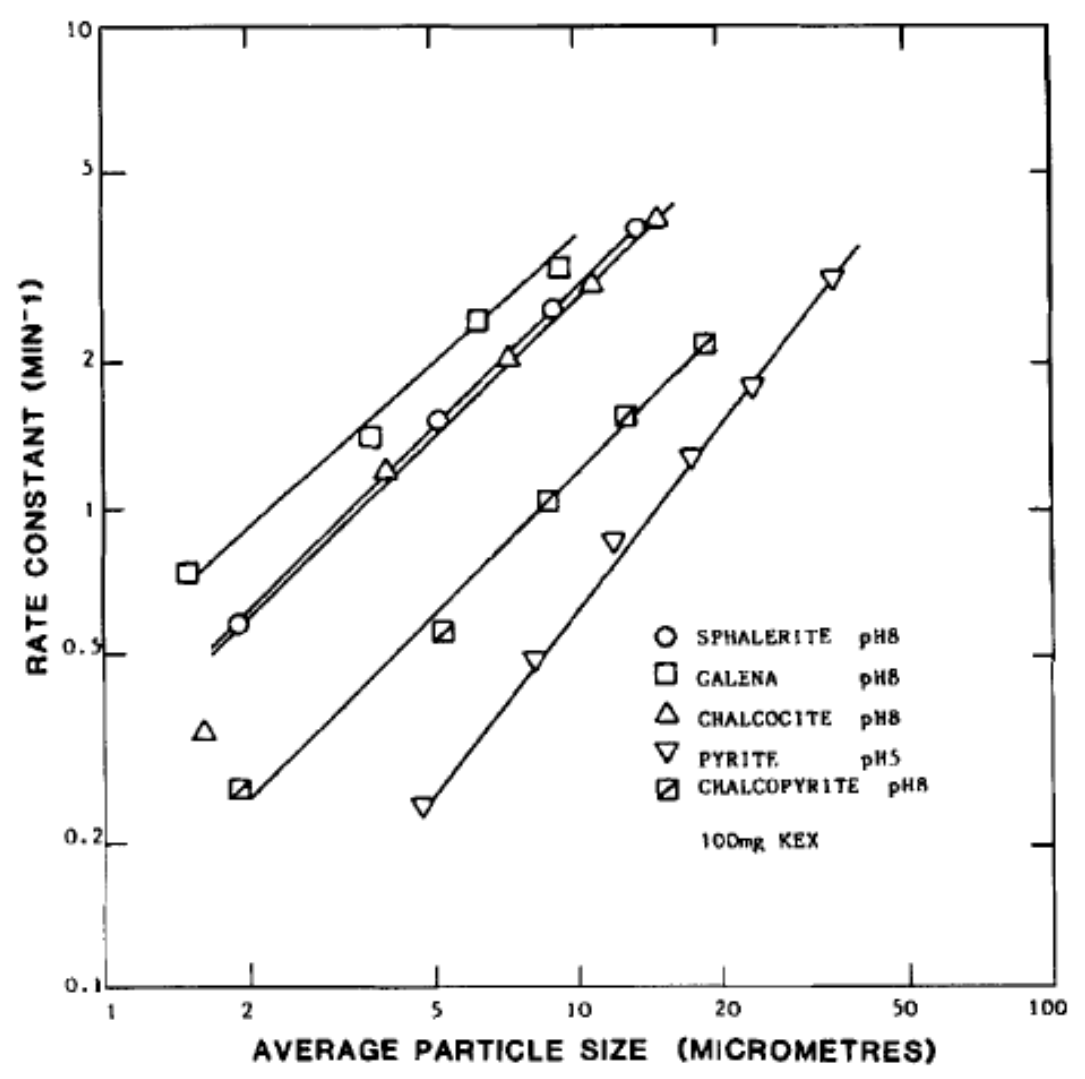

Figure 3.11: Flotation rate constant variation with particle size for batch flotation of several sulphide minerals. (Source: Ahmed \& Jameson, 1989)

As per the experimental results shown in Figure 3.11 the flotation rate constant $(k)$ and particle diameter $\left(d_{p}\right)$ have approximately a logarithmic relationship for 1-50 $\mu \mathrm{m}$ sized particles (Jowett, 1980; Ahmed and Jameson, 1989). Hence, froth flotation is usually effective over only a relatively narrow size range, typically 10-100 $\mu \mathrm{m}$, although for low density particles such as coal, flotation is routinely extended up to particles as large as $500 \mu \mathrm{m}$ (Jameson et al., 1977). 


\section{(b). The Effect of the Bubble Size on Flotation Rate}

Bennett et al. (1958) showed that the attachment efficiency increases with decreasing bubble size and provision of more bubbles. This was also proved by the Yoon and Luttrell model discussed above (Yoon \& Luttrell, 1985). It should also be noted that bubble size and turbulence are inter-connected in stirred cells (Ahmed \& Jameson, 1989).

\section{(c). Bubble Coalescence}

In bubbling systems, when small bubbles contact, they tend to coalesce to form larger bubbles to reduce the total surface area and the overall free energy of the system. In flotation systems, coalescence can be minimised or prevented through the addition of surfactants. Surfactants reduce the surface tension at the air-water interface and thus preserve small bubbles in the system. Lowering the surface tension also makes it easier to generate small bubbles. Coalescence in the froth regime of flotation columns has a significant direct impact on the circulation and cleaning process. There are different reasons why coalescence occurs in the froth. In the draining froth, coalescence occurs due to the drainage of the films between the closely packed bubbles. Gaudin (1957) suggested that coalescence is more probable when the bubble surface area is not covered by the mineral particles. Szatkowski (1995), suggested that hydrophobic particles promote increased bubble stability. However, a comprehensive study of coalescence phenomena is out of the scope of this research.

\section{(d). The Effect of Contact Angle}

Ralston et al. (1998) pointed out that for a given particle size, the attachment efficiency increases with increasing contact angle for a given particle size range. Surface active compounds can change the surface tension and increase the contact angle. Section 3.7 discusses this effect in detail. 


\subsubsection{Fine Particle Flotation}

The behaviour of fine particles or slimes, typically less than 10 microns, is different to that of larger particles of the same material. Flotation of fine particles encounters different limitations to those for coarse particles as mention in section 3.3.5.1. Owing to their small mass, fine particles lack kinetic energy (or momentum). Fine particles tend to follow the streamlines of the fluid suspension and so have a low collision probability. This behaviour, together with the high viscosity of the liquid medium in flotation cells, leads to poor flotation of these fine particles. Even when these fine particles collide, the momentum is often insufficient to rupture the liquid film, and in turn establish a stable three phase contact line. Due to this, the bubble-particle collision probability of fine particles is very low (Shahbazi et al., 2010). Also these particles have high specific surface areas and hence high adsorption capacities for chemical reagents. This will also lead to the low recovery of fine particles.

To obtain sufficient fine particle recovery through flotation new techniques have been developed (Miettinen et al., 2010). These techniques are based on enhancing bubbleparticle collision efficiency either by increasing apparent particle size or decreasing bubble size.

Apparent particle size can be increased by three main techniques. They are flocculation, coagulation and hydrophobic aggregation. In flocculation, flocs are formed by adsorption of polymers to minerals. Coagulation can be achieved through addition of electrolyte. With hydrophobic aggregation, the fine particles are selectively hydrophobised.

Decreased bubble size can be obtained using two main methods. They are mechanical method and physicochemical method. With the mechanical method, the shape of the rotor and stator can be changed or microporous material can be used at the bottom of the cell to produce gas bubbles. Zhou et al., 1997 proposed a method of generating tiny bubble by hydrodynamic cavitation. With this method gas bubbles are created in a liquid due to the rupture of a liquid-liquid or a liquid-solid interface under influence of external forces. The bubbles generated on a particle surface by cavitation naturally attach to the particle and hence increase the recovery of fine particles. 
Physicochemical methods include dissolved air flotation and electro-flotation. Under air flotation gas molecules are precipitated as small bubbles by changing pressure. Electroflotation use oxygen and hydrogen bubbles formed by the electrolysis of water.

However, so far none of these techniques have been able to provide a high recovery and there is still a need to search for better techniques.

\subsection{Particle Interactions through Air}

There are various mechanisms used to collect or separate particles dispersed in air. In this section a few of these techniques are discussed, covering wet scrubbing, granular filtration, recent research on film flotation, liquid marbles, and electrostatic precipitation.

\subsubsection{Wet Scrubbers}

Wet scrubbers are used to remove solid, liquid or gaseous contaminants from a gas stream by using water droplets. In scrubbing particulate matter from gases, the principal concern is usually in the removal of particles smaller than $20 \mu \mathrm{m}$ (Schnelle \& Brown, 2001). Wet scrubbers use a spray of liquid droplets to maximise the droplet surface area. Scrubbers are used extensively to control air-polluting emissions. Many different scrubber configurations have been designed using this concept. The separation efficiency of these systems depends on the circulation of liquid around the gas stream.

Successful design and operation of wet scrubbers depends on knowing the size and composition of particles to be collected. Particles suspended in the gas streams can be collected by water drops through mechanisms such as inertial impaction (impingement), interception, Brownian movement (diffusion deposition) and gravitational settling (Schnelle \& Brown, 2002). Among these effects Brownian movement is applicable for the very smallest of particles, less than $1 \mu \mathrm{m}$ in diameter. Inertial impaction and interception effects dominate in the $10-45 \mu \mathrm{m}$ size range. 


\section{Inertial Impaction Effect}

Most scrubbers use some form of inertial collection method as the primary collection system. Because of inertia, a particle moving in a gas stream can strike slowly moving or stationary obstacles (water drops) in its direct path, deviating from the gas streamlines as shown in Figure 3.12. Similarly in flotation, inertial forces deviate the particle trajectories from the water streamlines (Nguyen et al., 2003).

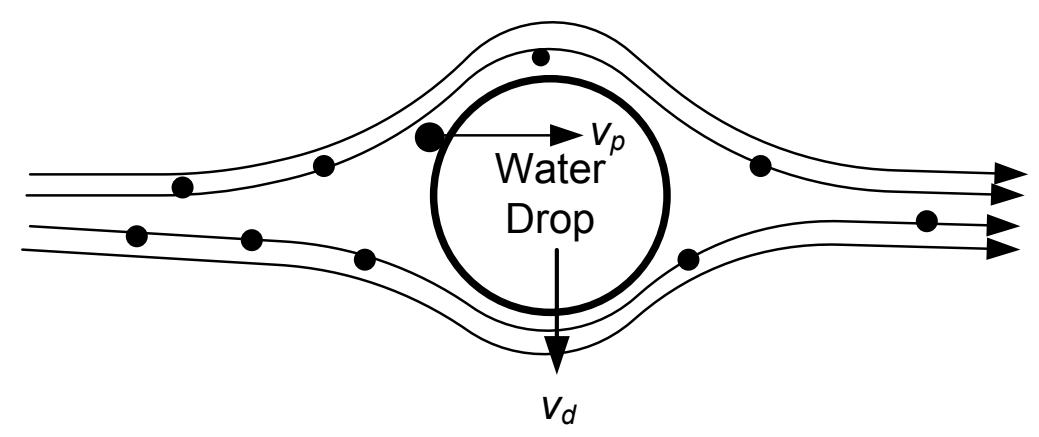

Figure 3.12: Inertial impaction of particles on a water drop.

The primary factors that affect the probability of an inertial impaction are particle size, particle density and the difference in velocity between the particle and the water drop. Larger, dense particles are collected more easily than smaller low density particles due to their greater inertia. Also, collection efficiency increases as the difference in velocity between the particle in the gas stream and the obstacle (or target) increases.

Albrecht and Sell (Burkoholz, 1989) have calculated an inertial impaction parameter $(\varphi)$ for a potential (ideal) flow. This is given in Equation 3.27.

$$
\varphi=\frac{\rho_{p} v d_{p}^{2} C u}{18 \mu D_{d}}
$$

where $\rho_{p}$ denotes particle density, $v$ denotes relative velocity of the particle with respect to the water drop (flow velocity), $d_{p}$ denotes the particle diameter, $C u$ denotes the Cuningham correction factor, $\mu$ denotes the gas viscosity and $D_{d}$ denotes the diameter of the water drop. 
This inertial impaction parameter $(\varphi)$ is a characteristic value for inertial separation (or efficiency of impaction). The inertial separation is directly proportional to the inertial parameter $(\varphi)$. The Cuningham slip correction factor accounts for molecular slip and this is applicable only for particles with a diameter less than $3 \mu \mathrm{m}$ (Environmental Protection Agency, 2010). Since we are focusing on particles with minimum average diameter of around $15 \mu \mathrm{m}$, this correction factor is not significant for the efficiency of our system.

\section{Effect of Interception}

In addition to the inertial effect, there is also the effect of interception. When the particle's trajectory overlaps the curve of the water drop as shown in the Figure 3.13 particles become attached to the water drop due to the interception effect.

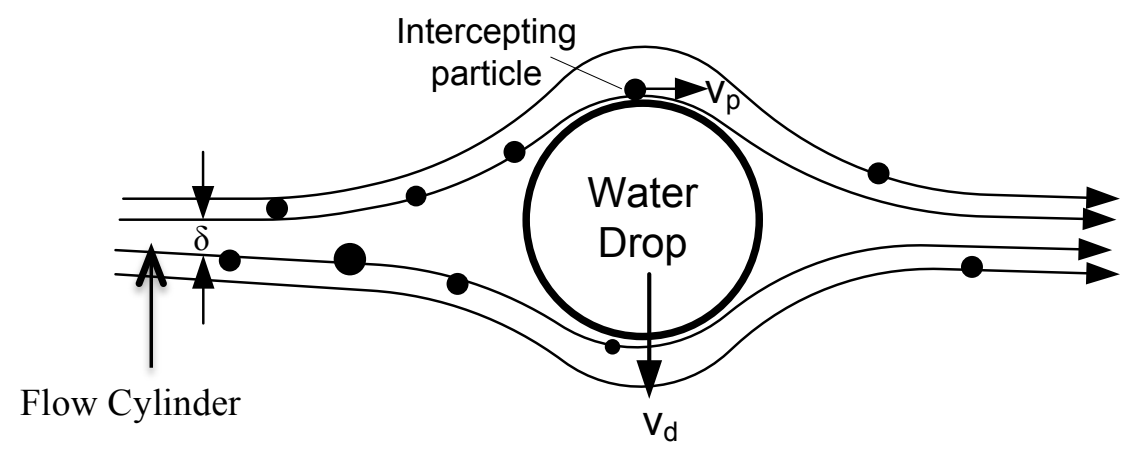

Figure 3.13: Interception Impaction of particles on a water drop.

The collection efficiency of particles by interception of water drops is a function of the particle diameter and drop diameter as given in Eq.3.28. That is,

$$
\eta=f\left(\frac{\delta^{2}}{D_{d}^{2}}\right)
$$

where $\delta$ represents diameter of the flow cylinder and $D_{d}$ represents the diameter of the water drop. This derivation is based on the gas flow around a cylinder (Burkoholz, 1989). Thus, as the water drop decreases, the collision efficiency increases. Studies 
have shown that $0.5 \mu \mathrm{m}$ fume particles need water droplets with diameters less than 100 $\mu \mathrm{m}$ for adequate collection (Nalco, 2009).

The other basic factors that determine the efficiency of wet particle scrubbers are the liquid/gas ratio, particle size, particle density and particulate affinity for water (wettability). Also formation of small water droplets with higher surface area will result in high capture efficiencies. The small particles, of lower momentum, fail to rupture the air film around the drops. Higher energy is required to scrub hydrophobic particles and less energy is required for hydrophilic particles. Efficiency of scrubbers can also be increased by reducing the surface tension of water (Nalco, 2009).

\subsubsection{Granular Filtration}

Granular filtration is a fluid-solid separation process commonly applied to remove small particles from various fluids (Tien \& Ramarao, 2007). This technology is basic to a large number of manufacturing industries (chemical, mineral, and food) as well as pollution abatement and environmental control. Granular filtration is commonly used for clarifying suspensions of dilute particle concentration.

In granular filtration, granules such as gravel, sand, pebbles and charcoal materials can be used as the filter medium. The fluid suspension is passed through the filter medium under pressure or gravity to remove fine particles. The fine particles in the fluid suspension are removed by their deposition onto the surface of the filter medium. Both liquid or gas streams can be treated with this method. Hence granular filtration can be referred as hydrosol or aerosol filtration depending on whether a liquid or gas suspension is involved. The most common application of the granular filtration is the use of sand filters for water purification.

In the granular filtration of hydrosols, particle deposition in the medium is effected through interception, sedimentation and Brownian diffusion with deposition taking place throughout the medium. For this reason, granular filtration of hydrosols is often referred to as deep-bed filtration or depth filtration. Inertial impaction is the main mechanism for aerosol collection in granular filtration of particles with diameters 
greater than $1 \mu \mathrm{m}$ and in the absence of applied external force. Granular filtration is characterized by its relatively high collection efficiency for particles covering a wide size range.

In addition to granular filtration other processes such as fibrous filtration, fabric filtration, centrifugal separation (cyclones), wet-scrubbing and electrostatic precipitation can be used to clarify solid-gas suspensions.

\subsubsection{Recent Research on Fine Particle Separation}

This research is concerned with the contacting of particles with a liquid via a continuous gas phase. Since the concept of selective collection is novel, very limited literature has been published. However, the basic methodology is similar to the process of wet particulate scrubbers (Section 3.4.1).

Recently two research groups have examined the potential of this concept as an alternative method of fine particle beneficiation. Tran et al. (2009) initiated the preliminary experimental studies on selective separation of ultra-fine particles less than 10 microns using fundamentals of film flotation while Galvin and Webber (2010) made some attempts to separate finer coal less than 90 microns using individual drops of water under a gas dispersion of fine coal and glass ballotini.

Tran et al. focused on separating fine hydrophobic graphite from hydrophilic quartz via direct contact with the air-water interface. They fed mixtures of these ultra-fine particles onto the water surface of the planar overflowing trough as shown in Figure 3.14. Drops of graphite and quartz particles suspension were added to the air-water interface through a burette. Immediate sinking of hydrophilic particles was observed while the hydrophobic particles remained at the water surface. The concentrate overflowed the lip of the container. 


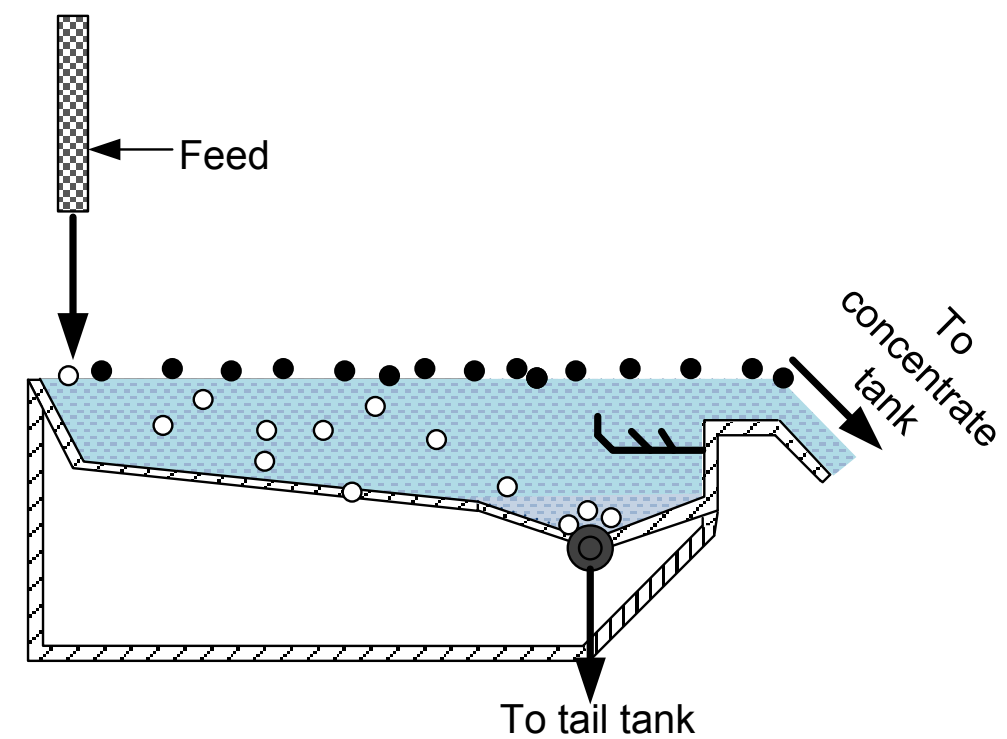

Figure 3.14: Inverse Flotation via overflowing trough.

In the initial batch experiments, concerned with the recovery of graphite from a graphite only suspension, $60 \%$ of the graphite was recovered. When the quartz only suspension was added, none of the particles were recovered from the over flow. For a mixture of particles, $90 \%$ of the graphite particles were recovered in the overflow and $85 \%$ of the quartz was found in the underflow. With the continuous system, involving graphite only suspensions, the recovery was $80 \%$, while the recovery for the quartz only suspensions was around $20 \%$ recovery via the overflow.

Though this is an efficient laboratory scale separation apparatus, there are a few problems. The main drawback is that the final product becomes moist. When the separated product is wet, it needs to undergo an extra drying process before the final usage. Also this system cannot be easily scaled up to an industrial scale due to the limited surface area, low feed rates and short lip length.

Meanwhile Galvin and Webber (2010) conducted experiments where they passed a drop of water through a gas dispersion of fine coal and silica in the less than $90 \mu \mathrm{m}$ size range. Initially they passed a water drop through a fine particle cloud of silica produced through a shaking screen. Under the microscope they observed tiny spherical particles of silica within the drop as shown in Figure 3.15 (a). Following the series of silica experiments, silica was replaced by fine coal on the same screen and a cloud of particles produced. There were a number of fine particles of the silica in the water drop, in spite 
of the fact that in excess of $99.9 \%$ of the feed particles were coal. The silica was a contaminant left over from the previous experiment. Moreover, there were no particles of coal to be found within the drop; however there was an aggregate of coal particles on the outer surface of the drop.

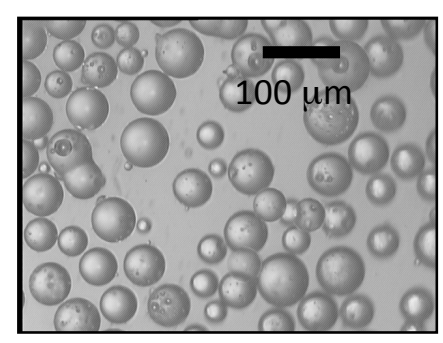

(a)

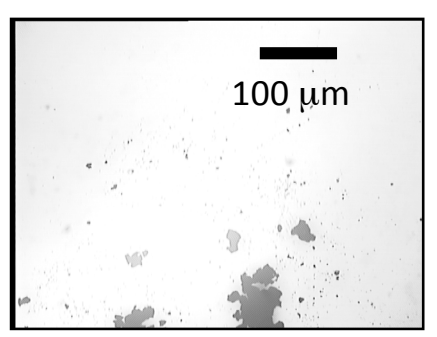

(b)

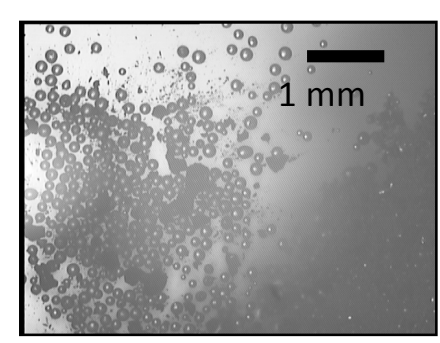

(c)

Figure 3.15: Microscope Images of water drops (a) Silica particles inside test drop (no coal in feed). (b) No coal particles found inside the test drop, though some coal evident at drop surface. (c) Silica particles inside drop (silica and coal feed). Images reproduced from Galvin et al, 2010.

Subsequent experiments involving fine coal produced drops containing no particles, though aggregates of coal were sometimes found at the surface of the drop. Figure 3.15 (b) shows one result. Finally, experiments were conducted using a mixture of silica and coal. Figure 3.15 (c) shows the fine particle silica inside a test drop, with some evidence of fine coal at the surface of the drop. These experiments have initiated the present work to further investigate this phenomenon.

\subsubsection{Liquid Marbles}

Liquid marbles are another example of collecting fine particles at the surface of a drop. Liquid marbles are generated by covering liquid drops with low surface energy micro or nano-particles (McHale \& Newton, 2011). In the literature, these are formed by rolling a liquid droplet on a hydrophobic powder (Aussillous \& QueÂre, 2001; Mahadevan \& Pomeau, 1999) as shown in Figure 3.16. 

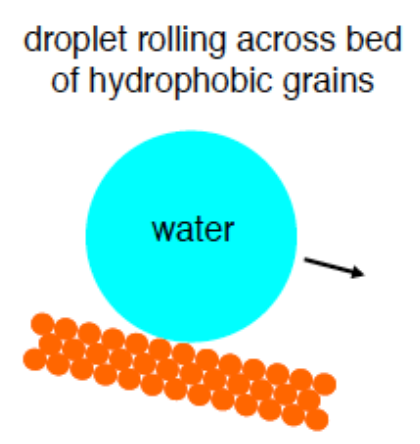
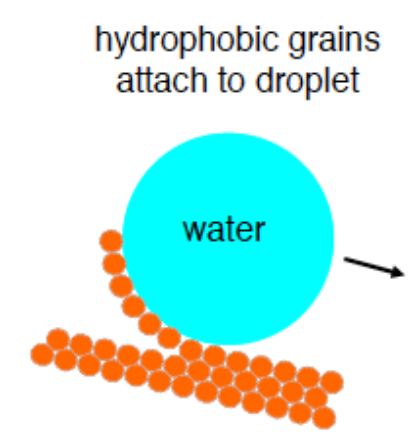

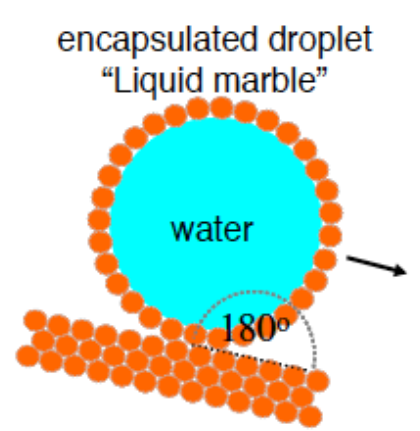

Figure 3.16: Self coating particles in Liquid Marble (Source: McHale and Newton, 2011)

However, recently it was observed that particles need not to be hydrophobic (contact angle, $\theta>90^{\circ}$ ) to remain attached to the surface of a water drop (McHale \& Newton, 2011). The grains of the solid randomly coat the drop initially and then spread around the whole surface of the drop.

These non-wetting soft solids can be transported easily (McHale \& Newton, 2011). Because the encapsulated liquid is coated with the particles, evaporation of the liquid is reduced. Liquid marbles also move easily across a surface without any contamination or leaving any residue. Other important features of liquid marbles include their ability to deform and spontaneous self-coating. Liquid marbles have several uses in droplet microfluidics since they can be merged, floated and divided, and their contents can be mixed. These micro reservoirs have been used in cosmetics (hydrophobic fumed silica as antiperspirants, moisturisers) and pharmaceutical industries (McHale \& Newton, 2011). The ability to use particle sizes ranging from tens of microns to nano-meters allows both opaque and transparent liquid marbles. Hence, liquid marbles are used in colorimetric sensing and optical applications. Further, the porous hydrophobic shell of liquid marbles supports gas transportation and hence can be used for gas sensing (Tian et al., 2010). 


\subsubsection{Electrostatic Applications}

Electrostatic applications depend on the attraction and repulsion of charged particles under the influence of an electric field (Ralston, 1961). Electrostatic precipitation, electrostatic coating and electrostatic separations (or High- tension separation) will be discussed in this section.

\section{(a). Electrostatic Precipitation}

Electrostatic precipitators remove dust particles from a gas by first ionising the gas. The gas ions collide with the dust particles imparting a charge to them (Cross, 1987). Large dust particles absorb a significant amount of charge. Once the charge is imparted similar charges are repelled by other gas ions. The charged particles are then attracted and deposited onto collecting plates where the charge is neutralised. When enough dust has accumulated, the dust particles fall off from the collector plates and collect at the bottom of the hopper. Depending upon dust characteristics and the gas volume to be treated, there are many different sizes, types and designs of electrostatic precipitators.

\section{(b). Electrostatic Coatings}

This is one of the main applications of electrostatics, where charged powder particles or paint droplets are sprayed onto an earthed material and coated with the paint (Moore, 1973). As illustrated in Figure 3.17, the spray gun atomises and charges the paint. The suspended paint particles or drops are charged to one electrical sign (negative) and the surface of the material to the other sign (positive). Then there is an attraction on the surface and the powder particles or droplets follow the electric field lines to form the coating. 


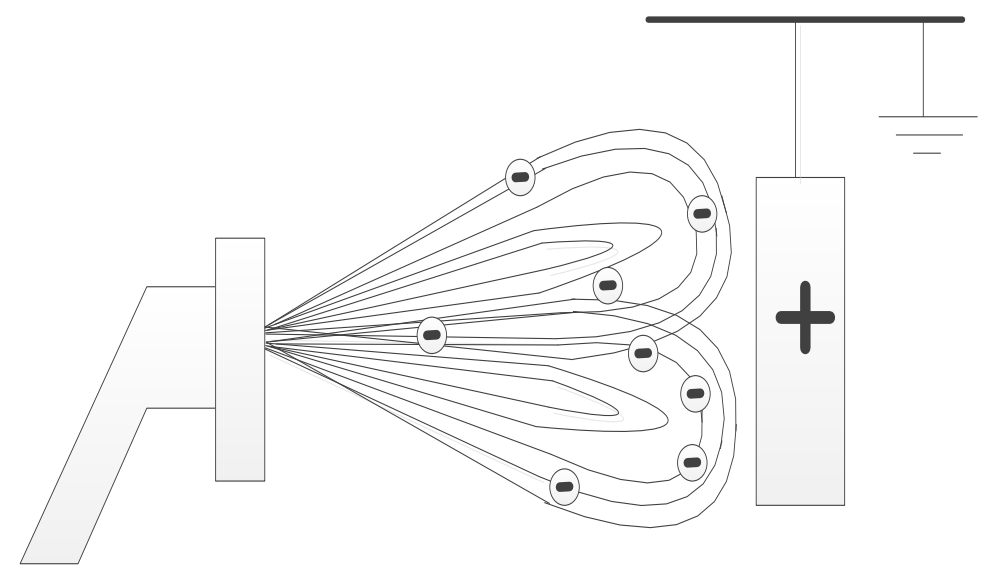

Figure 3.17: Electrostatic Coating.

\section{(c). Electrostatic Fluidised Beds}

An electrostatic fluidized bed is another application of powder coating with a high voltage direct current electrode above the porous plate to charge the fine powder particles (Moore, 1973). The principle of an electrostatic fluidised bed is shown in Figure 3.18.

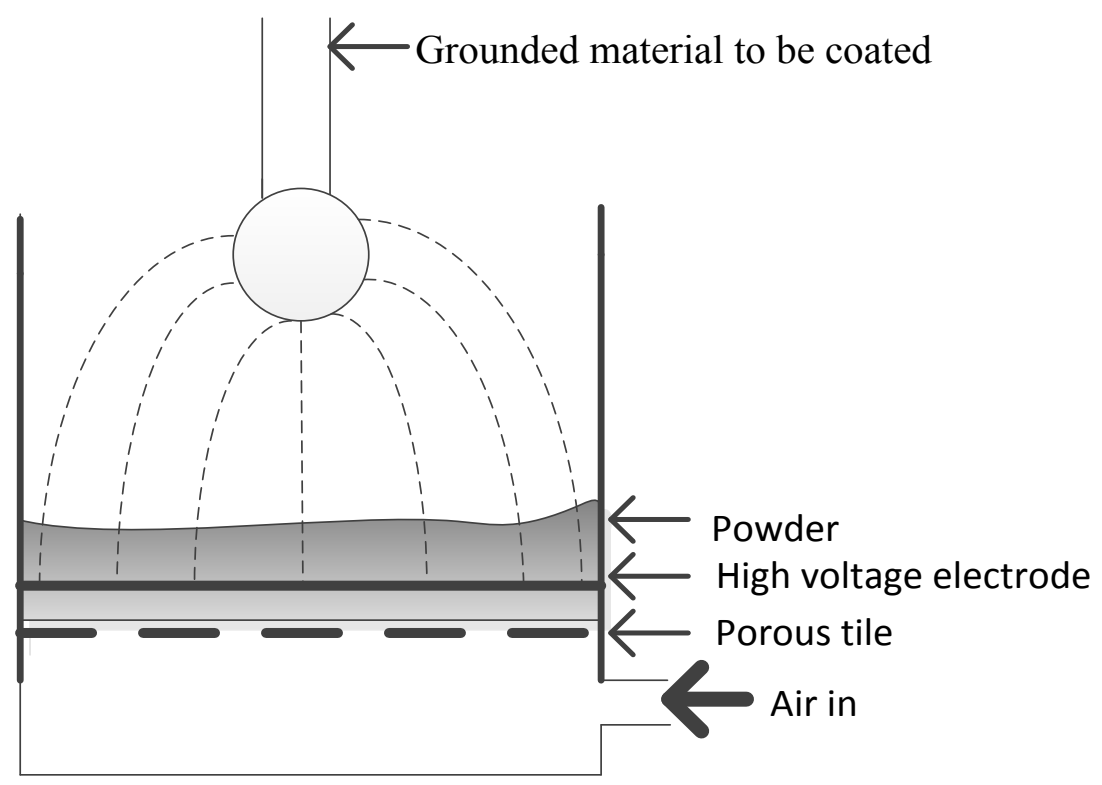

Figure 3.18: Simple electrostatic fluidised-bed coating system. 
The earthed substrate to be coated is held above the powder bed and particles are charged via the high voltage electrode. Once the particles are charged they lift from the bed as they are repelled by the electrode, and they repel each other, forming a cloud of powder above the electrode. These electrostatically charged particles are attracted to and coat materials that are at ground potential. Film thicknesses are similar to what can be achieved in the electrostatic spray process. The disadvantages of this method are that it is limited to few powders and inside corners of the object have low film thickness owing to the well-known Faraday cage effect. In a Faraday cage, an external static electrical field distributes the cage's conducting material in a way that they cancel the field's effect in the cage's interior. This results in low film thickness in inside corners.

\section{(d). Electrostatic Separation}

Electrostatic mineral separation methods depend on the particle charging properties, such as electrical conductivity or polarity of the frictional charge (Cross, 1987). Since particle charging methods were discussed in the second chapter, this section explains the separation applications.

Mineral separators, which use electrostatic charges to separate particles, can be categorised into two groups:

1. Electrodynamic (High tension) separators

2. Electrostatic separators

Electrodynamic separators or high tension separators produce separation by exploiting differences in the conductivity of particles and on the ability of the particles to develop surface charges. Electrostatic systems use little or no current flow while in electrodynamic systems use finite current flows.

In electrodynamic separators feed particles transport through a high voltage corona provided by the static electrode as shown in Figure 3.19 (Kelly \& Spottiswood, 1982). This ionises the feed particles. Conducting particles in the feed discharge their charges to the grounded drum rapidly while non-conductive particles retain their charges. Hence non-conductors are attracted to and held by the rotating drum while conductor particles 
fall off from the drum, separating the feed mixture. These devices are used for beach sands and iron ore separations.

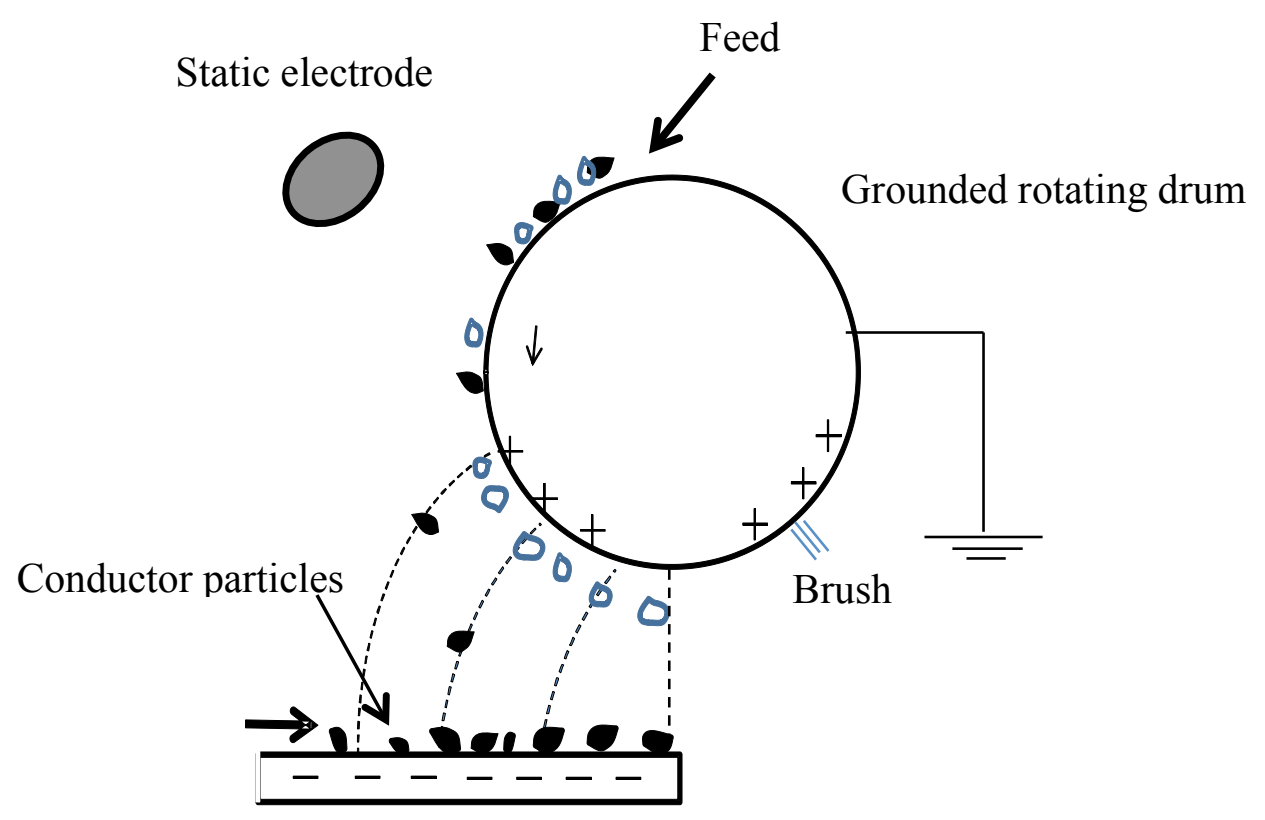

Figure 3.19: High tension electrostatic separator.

Irrespective of the charging technique, electrostatic separation of particles occurs only if the electrostatic forces acting on the particles are high enough to overcome the gravitational and inertial forces.

In electrostatic separators a charge is induced in conductive particles by the strong electric field while non-conductive particles remain uncharged. The electric field lifts the charged particles from the plate and thus separates them from non-conducting particles. The falling trajectories of these conducting particles depend on the discharge rate of particles (Kelly \& Spottiswood, 1982).

Triboelectric separation, applicable to non-conducting particles, is an example of electrostatic separation, based on differences in the surface charges of various materials. 


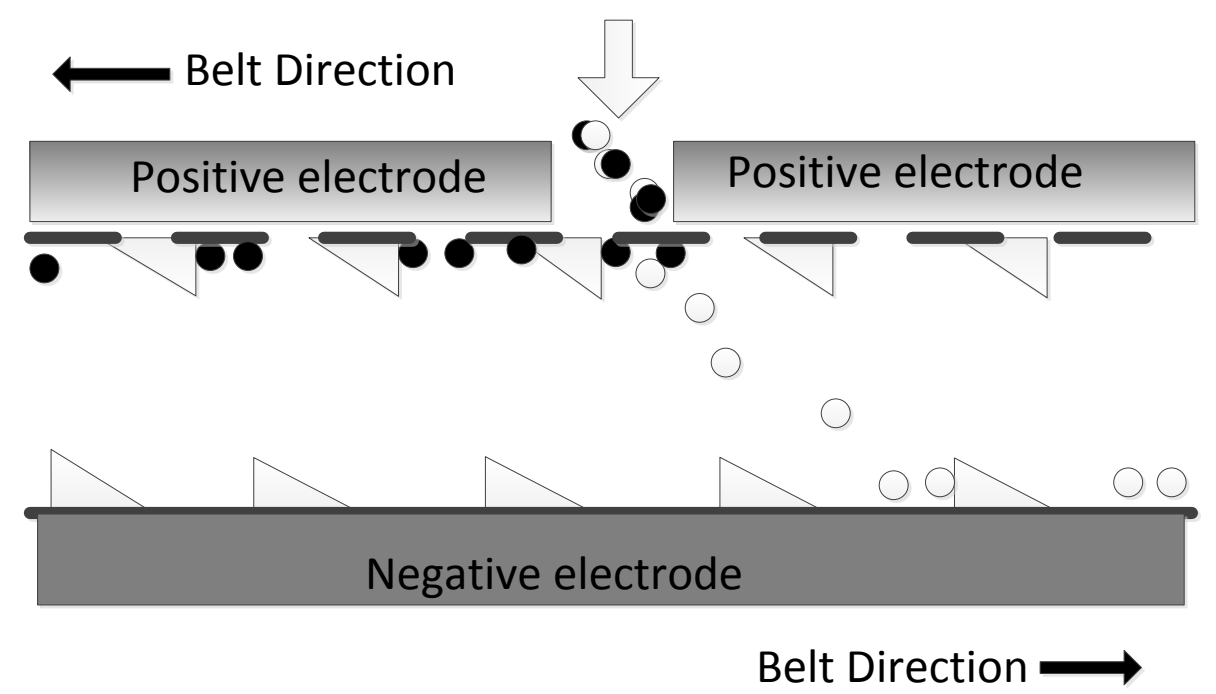

Figure 3.20: Separation process of a triboelectric separator.

As shown in Figure 3.20, particles are fed into a thin gap between two parallel planar electrodes where they acquire opposite charges through inter-particle contact. Charged particles are attracted to the electrode plate of opposite charge as the belts move.

Two types of electrostatic separations are electrophoresis and dielectrophoresis (Kelly $\&$ Spottiswood, 1989). They are based on differences in electrical resistivities and differences in electronic surface structure. Electrophoresis involves charged particles while dielectrophoresis involves uncharged particles. In electrophoresis the electric field can be produced either from a high voltage source or a charged particle's own electric field. Electrophoresis separation involves pre-charged particles using a high intensity electric field. Dielectrophoresis involves the movement of neutral particles which are polarisable in a non-uniform electric field.

Electrostatic separation is commonly used for particles below $1 \mathrm{~mm}$. Electrostatic separation methods require dry surfaces for effective separation as surface moisture can alter the electrical conductivity of the particles. In some cases, moisture acts as a binder for particles. 


\subsection{Summary}

Hydrodynamics explain the theories of particle movement in fluids. The main forces acting on particles within fluids are gravitational, buoyancy and drag. There are three different hydrodynamic flow regimes, laminar, intermediate and turbulent. Different forces, especially inertial and viscous, dominate the particle behaviour in each of these flow regimes.

Flotation is a major application of particle-gas interactions through a continuous liquid phase. Flotation is applied to beneficiate different mineral types such as sulphur, coal, copper, phosphates as well as carbonates, with the development of various flotation machines and reagents. The basic steps of flotation are bubble-particle collision, attachment and detachment. These processes have different probabilities, which affect the ultimate recovery of particles in flotation. Flotation kinetics quantifies the parameters that control the flotation process.

Various applications of particle interactions through air, there were several selected applications discussed in detail. These included wet scrubbing, granular filtration, and recent film flotation techniques, formation of liquid marbles and electrostatic separators. These processes have some relevance to the selective collection separation of particles in air, a key focus of this study. The design concepts and operating variables of these applications were outlined in order to provide the background needed for this study. 


\section{Chapter 4}

\section{MODEL DEVELOPMENT}

\subsection{Introduction}

\subsection{A Model for Drop-Particle Collision}

4.2.1 Drop-Particle Collision Model for $u_{d} \gg u_{p}$

4.2.2 Drop-Particle Collision Model for $u_{p} \gg u_{d}$

\subsection{Discrete Element Model}

\subsection{Discussion}




\section{Journal Article Relevant to the Chapter and Author Contributions}

Paper: Selective Collection of Fine Particles by Water Drops

\section{Contribution:}

\begin{tabular}{|l|l|}
\hline Author & Primary Contribution \\
\hline Liyanaarachchi, K.R & $\begin{array}{l}\text { Lead author, experimentalist, analysis of experimental } \\
\text { work and collaborator in the design ensuring well } \\
\text { defined experimental conditions, collaborator in } \\
\text { developing the analytical model }\end{array}$ \\
\hline Webber, G.B & $\begin{array}{l}\text { Co-supervisor and co-developer of the experimental } \\
\text { design }\end{array}$ \\
\hline van Netten, K. & Co-developer of the DEM Model \\
\hline Moreno-Atanasio, R. & Co-developer of the DEM Model as the supervisor \\
\hline Galvin, K.P & $\begin{array}{l}\text { Principal supervisor, primary developer of the } \\
\text { analytical model and co-developer of the experimental } \\
\text { design }\end{array}$ \\
\hline
\end{tabular}

Kevin Galvin

Kathika Liyanaarachchi 


\subsection{Introduction}

This study is concerned with the interaction between a gaseous dispersion of fine particles travelling in the horizontal direction and discrete drops of water falling vertically through the dispersion. A simple analytical model of the particle-drop collision was developed to describe the particle recovery by the drops as a function of the water flux, covering two extremes of relative velocity between the particles and drops. These results were compared with a simulation based on the Discrete Element Method (Liyanaarachchi et al., 2014). Further validations were obtained from the experimental results discussed in Chapter 6 .

\subsection{A Model for Drop-Particle Collision}

The basis for a selective collection between hydrophilic and hydrophobic particles is best characterised in terms of wetting and adhesion between the particles and water drops. Particle adhesion is governed primarily by the lowering of the surface energy, and is further influenced by the surface charge. The thermodynamic criterion governing the potential for drop-particle attachment is the contact angle at the line formed by the intersection of the three phases (Adamson, 1997). If the contact angle has a high value the solid is described as hydrophobic, while if the contact angle is relatively small the solid is described as hydrophilic.

In this chapter, two analytical models for describing the interception of falling water drops with a dispersion of fine particles are developed. It is noted that the models assume that each collision between a hydrophilic particle and drop results in particle attachment to, and subsequent engulfment in the drop. For simplicity, the trajectories of the particles are assumed to follow the gas, and hence it is assumed the falling drops have no effect on the particle trajectories. These models are assessed in the later experimental section in Chapter 6. The first model is based on the assumption that the horizontal velocity of the particles, $u_{p}$, across the collision chamber is relatively low compared to the fall speed of the drops, $u_{d}$. This is most likely to be true once the drops have accelerated to their full terminal velocity. The second model, described later, 
addresses the early phase, when the drops are first released. Here their velocity is significantly lower than their terminal velocity, and thus it is assumed the drop fall speed is much less than the horizontal velocity of the particles.

The theoretical collision zone where the particles and drops interact, shown in Figure 4.1, has an active horizontal cross-section of $X Y$, where $X$ is the distance along the zone, parallel with the flowing particles, and $Y$ is the distance across the zone. The collision zone has an active vertical cross-section of $Y Z$, where $Z$ is the height of the zone parallel with the falling drops. It is noted that the distance, $X$, along the zone is divided into $N$ rows of drops. Each row, of width $Y$ and depth $X / N$, is the source of $m^{\prime}$ drops, and thus the grid provides $m^{\prime} N$ sources for drop formation above the collision zone.

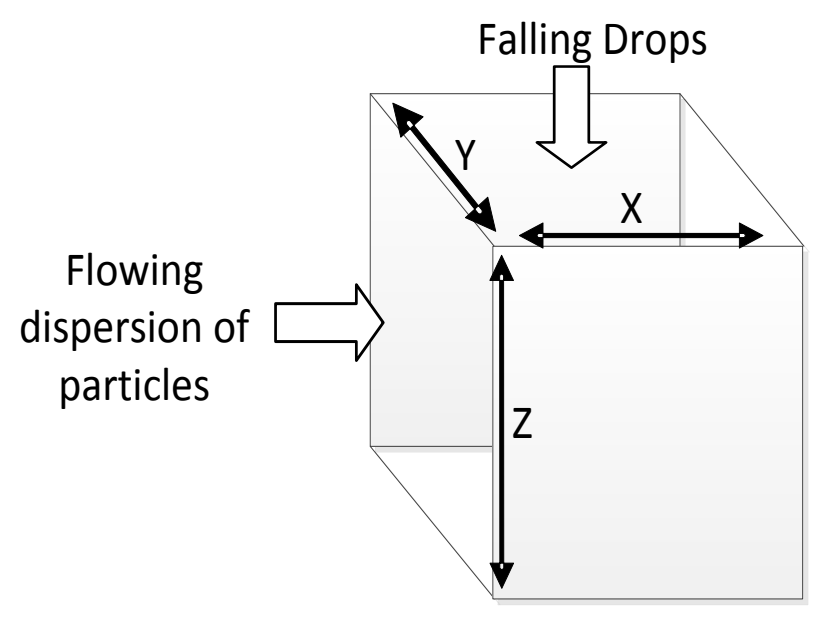

Figure 4.1: Schematic representation of the theoretical collision zone showing the dimensions, and the direction of flux of the particles (horizontal) and water drops (downward).

The water volume flux, $W\left(\mathrm{~m}^{3} /\left(\mathrm{m}^{2} \mathrm{~s}\right)\right)$, is based on the cross-sectional area, $X Y$ while the particle volume flux, $G\left(\mathrm{~m}^{3} /\left(\mathrm{m}^{2} \mathrm{~s}\right)\right)$, is based on the cross-sectional area, $Y Z$. 


\subsubsection{Drop-Particle Collision Model for $\boldsymbol{u}_{d} \gg \boldsymbol{u}_{p}$}

In this section it is assumed the horizontal particle velocity, $u_{p}$, is relatively low compared to the drop fall speed, $u_{d}$. The volume fraction of the particles, $\phi_{p}$, is given by the feed volume flux of particles, $G_{f}$, divided by the horizontal particle velocity, $u_{p}$. This particle velocity is assumed to be the same as the air flow velocity in the horizontal direction that is responsible for particle movement. Thus, $\phi_{p}=\frac{G_{f}}{u_{p}}$. The volume swept by the falling drops is now applied to the volume fraction of the particles in order to calculate the volume of the particles recovered through collision.

In a time, $d t$, the drops fall a distance $d Z$ as shown in Figure 4.2, hence the volume swept by one falling drop in time $d t$ is $d v$,

$$
d v=A_{d} d Z
$$

The projected disc area corresponding to a water drop having a diameter $D_{d}$ is, $A_{d}=\frac{\pi}{4} D_{d}^{2}$, while $V_{d}=\frac{\pi}{6} D_{d}^{3}$ is the volume of the spherical drop. The number of drops in $1 \mathrm{~m}^{3}$ is,

$$
n_{d}=\frac{\phi_{d}}{V_{d}}
$$

where $\phi_{d}$ is the volume fraction of the drops. Therefore the total volume per $\mathrm{m}^{3}$ swept out in $1 \mathrm{~m}^{3}$ in a time, $d t$, is,

$$
d V=\left(\frac{\phi_{d}}{V_{d}}\right) A_{d} d Z
$$




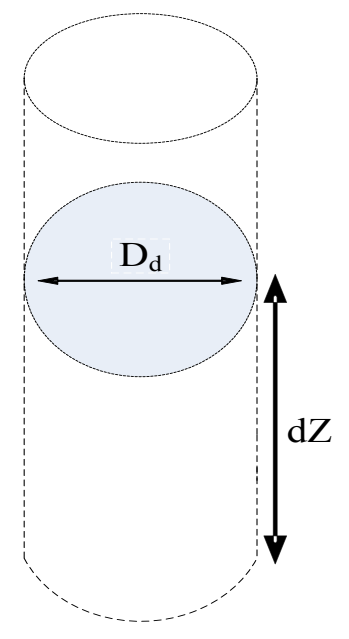

Figure 4.2: Schematic representation of the volume swept by a falling drop, that may be approximated by a cylinder with diameter equal to the drop, $D_{d}$, and height of $d Z$, the distance the drop falls in time $d t$.

The volume of particles that collide with this swept volume per $\mathrm{m}^{3}$ in a time, $d t$, is,

$$
d V_{p}=\phi_{p} d V=\phi_{p}\left(\frac{\phi_{d}}{V_{d}}\right) A_{d} d Z
$$

Note that the steady state volume fraction of the drops is, $\phi_{d}=\frac{W}{u_{d}}$ where $u_{d}$ is the drop fall velocity $\left(u_{d}=\frac{d Z}{d t}\right)$ and $W$, the flux of water. Therefore the volume of particles that collide per second in $1 \mathrm{~m}^{3}$ is,

$$
\frac{d V_{p}}{d t}=\phi_{p}\left(\frac{W}{u_{d}}\right)\left(\frac{1}{D_{d}}\right)\left(\frac{3}{2}\right) u_{d}=\frac{3}{2} \phi_{p} \frac{W}{D_{d}}
$$

The volume flux of particles that collide is,

$$
G_{c}=\frac{d V_{p}}{d t} \frac{(X Y Z)}{(Y Z)}=\frac{3}{2} \frac{\phi_{p} W}{D_{d}} \frac{(X Y Z)}{(Y Z)}
$$

Thus, the volume flux of particles that collide is,

$$
G_{c}=\frac{3}{2} \frac{\phi_{p} X W}{D_{d}}
$$


where the collision volume flux is also given by,

$$
G_{c}=V_{p} \frac{d N_{p}}{d t}
$$

Note that $N_{p}$ is the number flux of particles, $\frac{d N_{p}}{d t}$ the rate per unit area at which the particles collide, and $V_{p}$ the volume of a particle. As already noted, the feed volume flux is $G_{f}=\phi_{p} u_{p}$ where $u_{p}$ is the horizontal velocity of the particles, which is equal to the gas velocity, i.e. the gas flux. Thus we can eliminate the volume fraction, $\phi_{p}$, of the particles. Therefore, the volume collision flux is,

$$
G_{c}=\frac{3}{2} \frac{G_{f}}{u_{p}} \frac{X W}{D_{d}}
$$

and hence the recovery via collision is,

$$
R=\frac{G_{c}}{G_{f}}=\frac{3}{2} \frac{X}{u_{p}} \frac{W}{D_{d}}
$$

\subsubsection{Drop-Particle Collision Model for $u_{p} \gg u_{d}$}

We now consider the other extreme, in which the horizontal particle velocity, $u_{p}$, is much greater than the drop fall velocity, $u_{d}$. With $u_{p} \gg u_{d}$, an instantaneous silhouette of the falling drops provides a measure of the free space through which the high speed particles can travel, and the remaining space that leads to collisions and hence recovery. Hence we assume the particle recovery is equal to the total fraction of the two dimensional image that is covered by drops. Given the drops are formed along a series of parallel rows the best approach is to consider the first row of drops in isolation. The distance, $X$, in Figure 4.1 is therefore divided into smaller lengths, such that $x=X / N_{R}$, where $N_{R}$ is the number of rows of drops. The relevant volume of space associated with the first row of drops, $Q$ is; 


$$
Q=x Y Z
$$

and the steady state volume fraction of drops within this zone is,

$$
\phi_{d}=\frac{W}{u_{d}}
$$

For $N$ drops, the volume of drops within the zone is,

$$
N \frac{\pi}{6} D_{d}^{3}=\phi_{d} Q
$$

The corresponding projected area of these drops is,

$$
N \frac{\pi}{4} D_{d}^{2}=\frac{3 \phi_{d} Q}{2 D_{d}}
$$

Therefore the fraction of the two dimensional projected area covered by drops is,

$$
\alpha=\frac{N \frac{\pi}{4} D_{d}^{2}}{Y Z}=\frac{3 \phi_{d} x}{2 D_{d}}
$$

Incorporating equation 4.12 into equation 4.15 gives,

$$
\alpha=\frac{N \frac{\pi}{4} D_{d}^{2}}{Y Z}=\frac{3 \times W}{2 u_{d} D_{d}}
$$

The recovery of the particles through collision with the first row of falling drops is $R=\alpha$, hence the portion reaching the second row of drops is $(1-\alpha)$. Similarly, the recovery from the second row of drops is $\alpha(1-\alpha)$, therefore the portion passing through is $(1-\alpha)^{2}$. In general, the recovery is

$$
R=1-(1-\alpha)^{N_{R}}
$$


Applying the binomial approximation and setting $X=x N_{R}$, the recovery becomes,

$$
R=\frac{3}{2} \frac{X}{u_{d}} \frac{W}{D_{d}}
$$

Equation 4.18, which is based on the assumption, $u_{p} \gg u_{d}$, is governed by the drop velocity $u_{d}$. This result is virtually identical to equation 4.10 , which applies to the opposing condition, $u_{d} \gg u_{p}$, expressed in terms of the particle velocity, $u_{p}$. Both these models show the recovery is proportional to the water flux (product of fall speed and hold-up) and inversely proportional to the diameter of the drops.

It is noted that the drops commence their motion from a state of rest, as they detach from their source, and accelerate towards their terminal velocity. Thus the relevant drop velocity, which is lower than the terminal velocity $(1.4 \mathrm{~m} / \mathrm{s}$, refer to Appendix C), must be calculated with regards to the inertial forces. For this study, half the total average fall distance, $s=Z / 2$, is only $0.055 \mathrm{~m}$, hence there is insufficient time for significant drag force to develop. Thus, Newton's laws of motion provide an accurate measure of the drop fall speed at the half-way point. That is,

$$
u_{d}=\sqrt{2 g s}
$$

where $g$ is the acceleration due to gravity. Equation 4.19 gives $u_{d}=1.04 \mathrm{~m} / \mathrm{s}$. The problem is assumed to be linear either side of the half-way point, hence this velocity is adequate.

Both models provide the recovery due to collision in terms of the distance $X$ along the collision zone. These models should be valid for relatively low recovery values corresponding to short distances $X$. A differential analysis over a small distance, $d X$, based on the fraction of particles that escape the falling drops, leads to a more general result valid at higher recovery. 
For $u_{d} \gg u_{p}$ the recovery becomes

$$
R=1-\exp \left(-\frac{3}{2} \frac{X}{u_{p}} \frac{W}{D_{d}}\right)
$$

while for $u_{p} \gg u_{d}$

$$
R=1-\exp \left(-\frac{3}{2} \frac{X}{u_{d}} \frac{W}{D_{d}}\right)
$$

Experimental system values of $X=0.0235 \mathrm{~m}, u_{p}=6 \mathrm{~m} / \mathrm{s}$ (used for $\left.u_{d} \gg u_{p}\right), u_{d}=1.04$ $\mathrm{m} / \mathrm{s}$ (used for $u_{p} \gg u_{d}$ ), $D_{d}=0.003 \mathrm{~m}$ and values of the water flux, $W$, (given in Table 4.1), were substituted into the two models to obtain the estimated recoveries shown in Figure 4.3.

Table 4.1: Calculation of the water flux based on experimental values of the water flow rate. These values of $\mathrm{W}$ were used to estimate the recovery values shown in Figure 4.3.

\begin{tabular}{|c|c|c|}
\hline Water Flow Rate \% & Volumetric Rate (L/min) & Water Flux $\left(\mathrm{m}^{3} / \mathrm{m}^{2} \mathrm{~s}\right)$ \\
\hline 30 & 0.65 & 0.0057 \\
\hline 50 & 1.00 & 0.0088 \\
\hline 70 & 1.35 & 0.0118 \\
\hline 85 & 1.63 & 0.0143 \\
\hline 100 & 1.90 & 0.0167 \\
\hline
\end{tabular}




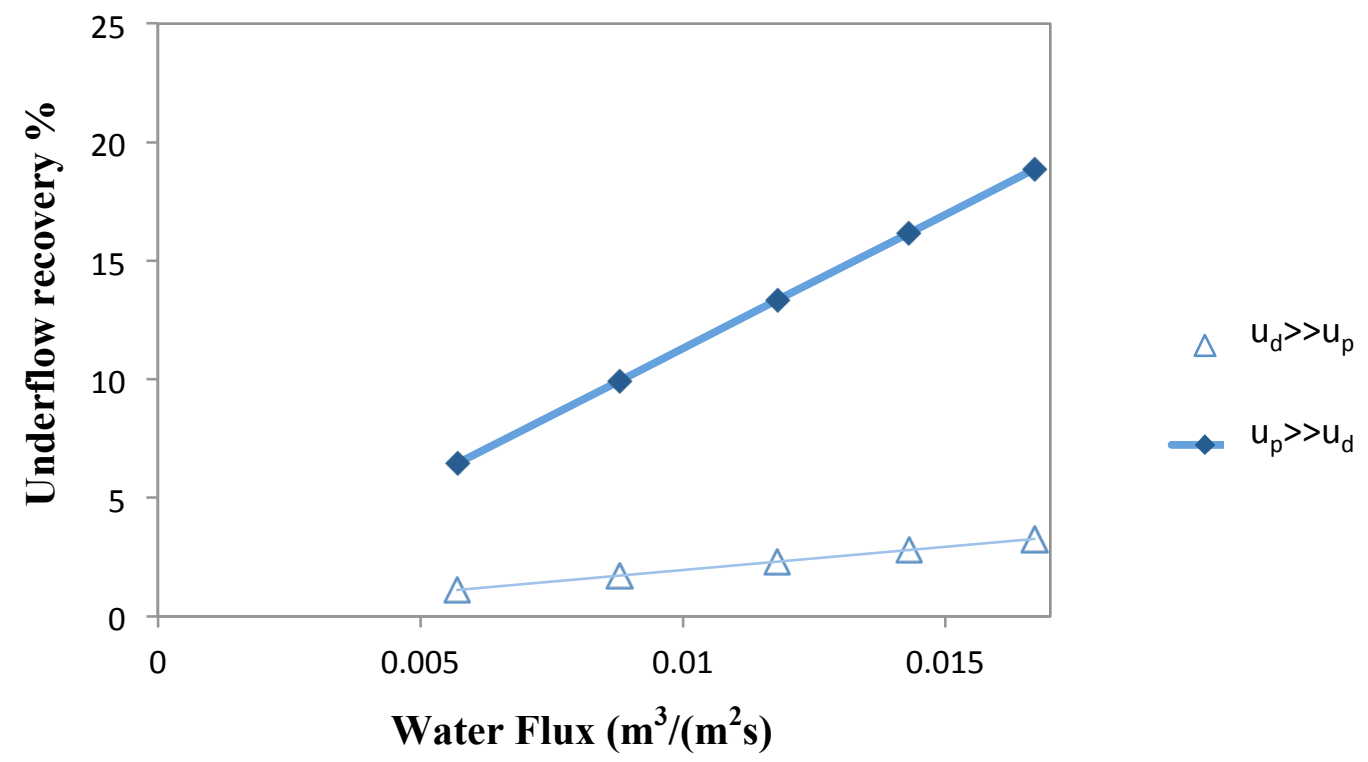

Figure 4.3: Estimated particle collision recoveries obtained using the water flux values given in Table 4.1. The two different models were used with $X=0.00234 \mathrm{~m}, D_{d}=0.003$ $\mathrm{m}, u_{p}=6 \mathrm{~m} / \mathrm{s}$, and $u_{d}=1.04 \mathrm{~m} / \mathrm{s}$.

As shown in the Figure 4.3 drop-particle model for $u_{p} \gg>u_{d}$ gives in a higher recovery compared to recovery of $u_{d} \gg u_{p}$ model. Hence, it is noted that the particle velocity $\left(u_{p}\right)$ should be higher than the drop velocity $\left(u_{d}\right)$ for a high underflow recovery.

\subsection{Discrete Element Model}

The simulation model is based on the experimental system. At the beginning of the simulations drops were positioned at the top of the system and distributed randomly in the $Y$ direction and in parallel lines along the $X$ direction (Fig. 4.1). Particles were randomly positioned along the $Y Z$ left plane (Fig. 4.1). An initial horizontal velocity was given to the particles while drops were released with zero initial velocities. The capture of the particles was analysed by recording the number of particles attached to the drops. 
Figure 4.4 shows a three dimensional plot of the recovery with different water flux and drop diameters according to Eq.4.10. As shown in this graph recovery increases with the reducing drop diameters and increasing water flux.

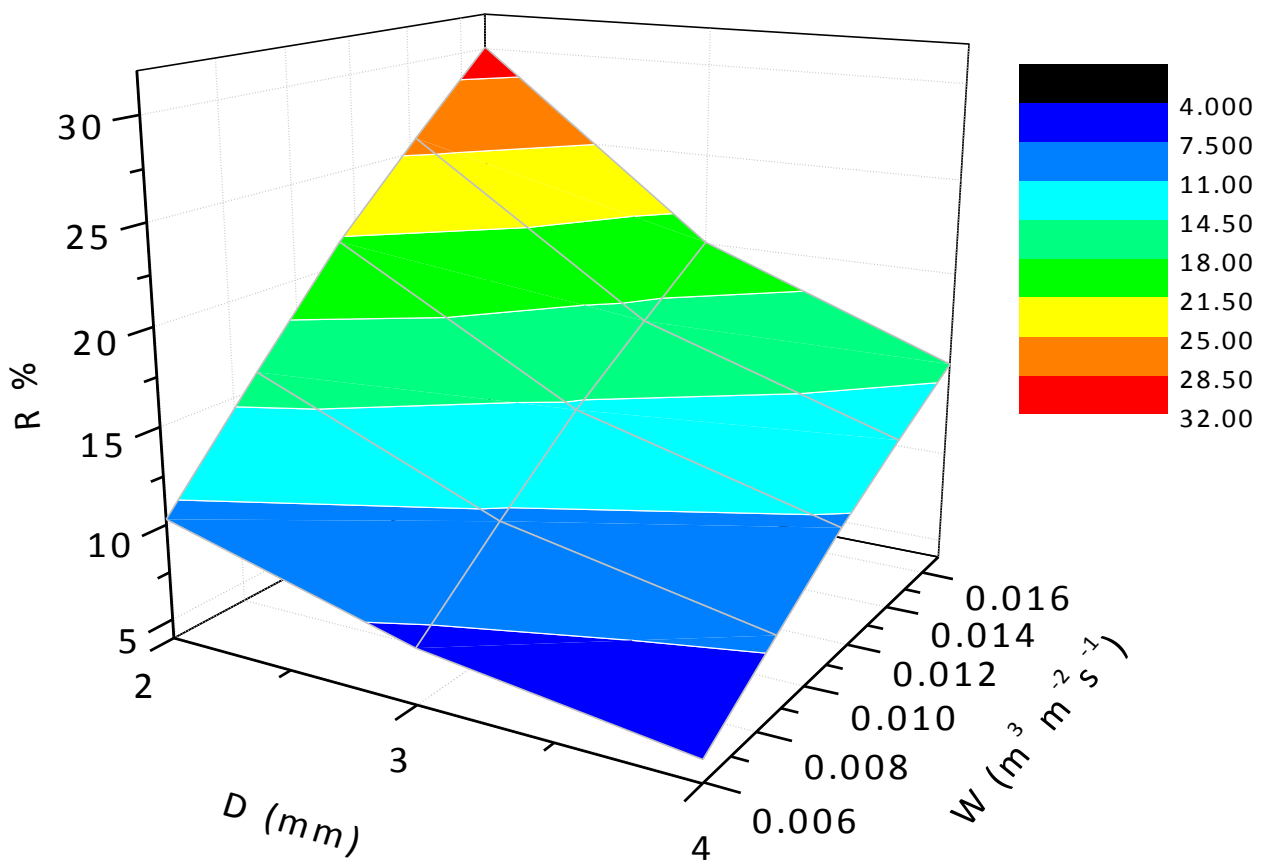

Figure 4.4: Three dimensional graph showing the recovery variation with the water flux and the drop diameter assuming $u_{p}>>u_{d}$. R represents the underflow recovery, D represents the drop diameter and $\mathrm{W}$ represents the water Flux.

\subsection{Discussion}

The main purpose of the models is to establish a basic understanding of the major, first order, parameters that govern the capture or particle recovery. Hence one goal is to experimentally validate this model. The intrinsic recovery given by Equations 4.10 and 4.18 is based on the assumption that every particle that intercepts a falling drop, however slightly, is captured. Thus the equation provides, in principle, an upper limit on performance and an appreciation of the basic parameters that matter. Selectivity can 
then be assessed in terms of the measured recovery of one particle species relative to another under identical conditions.

The Discrete Element Method (DEM) was applied to this problem by co-workers VanNetten and Moreno-Atanasio (Liyanaarachchi et al., under review). This model was used to validate the two analytical models described above. The DEM model was also applied to the intermediate conditions, which involve particle and drop velocities of a comparable magnitude. The DEM was developed by assigning material properties and sizes to each individual particle within the system. The particle and drop motions were in turn described using Newtons's law. Elastic properties were also assigned to both the particles and the drops. The contact elasticity was simulated using the Hertz law. A contact adhesion force between the particles and the drops, equal to $1.0 \times 10^{-5} \mathrm{~N}$, was also used. Figure 4.5 shows the simulated recovery based on different particle velocities.

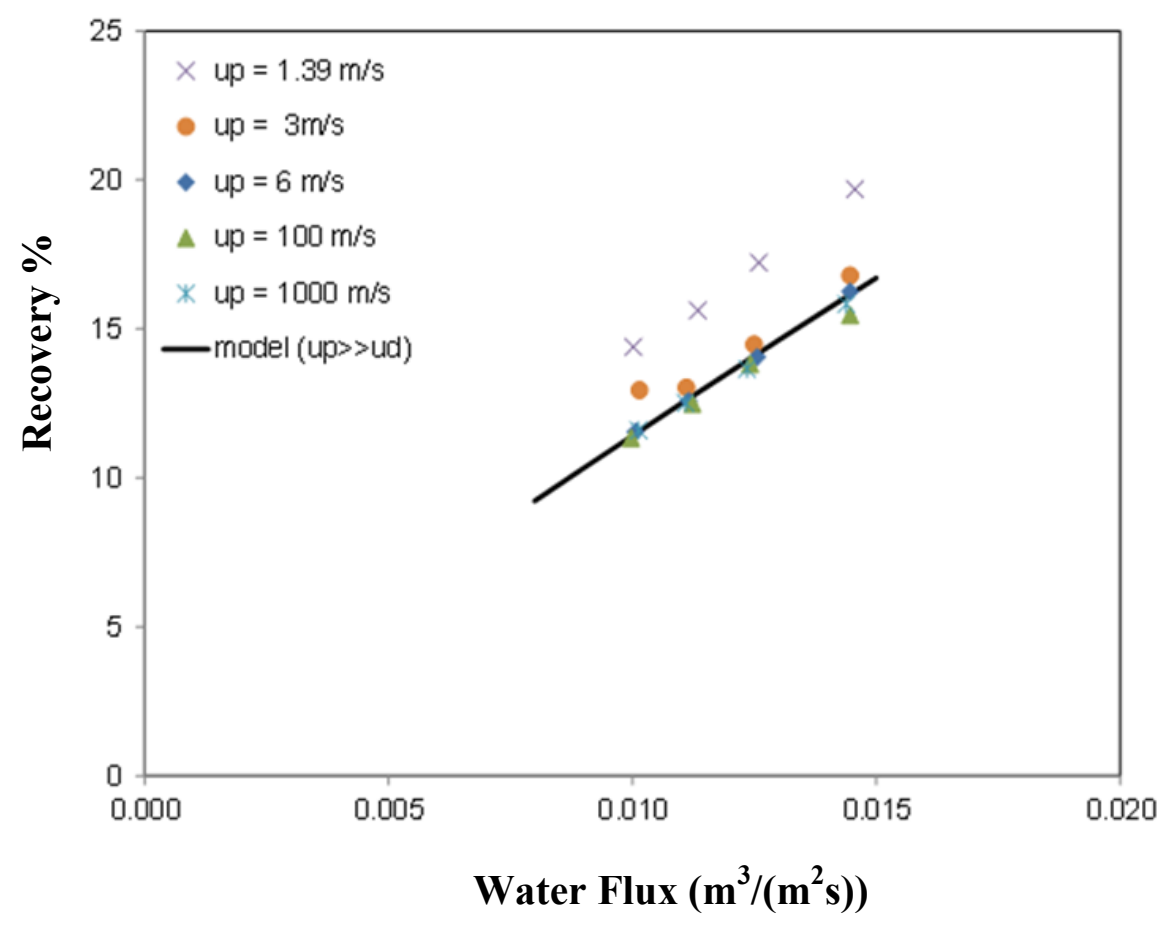

Figure 4.5: Simulated and estimated recovery as a function of the water flux. It is evident that the recovery values converge towards the idealised model when the particle velocity greatly exceeds the drop velocity. 
As the particle velocity increased, for a fixed drop velocity, the solution shifted towards an asymptotic solution valid for very large particle velocities. The asymptotic result is in excellent agreement with the analytical model, applicable to very high particle velocity, well in excess of the drop velocity.

However in conclusion, the analytical method provided a sound basis for the design of the experimental set up. 


\section{Chapter 5}

\section{EXPERIMENTAL METHOD \\ FOR SELECTIVE COLLECTION OF FINE PARTICLES}

\subsection{Introduction}

\subsection{Preliminary Single Drop Experiments}

\subsection{Development of Multiple-Drop Experimental System}

5.3.1 Feeding Configuration

5.3.2 Collision Chamber and the Vacuum Pump

5.3.3 Water Distribution Chamber Design and Cyclone

\subsection{Experimental Methodology and Materials}

\subsubsection{Methodology and Materials for Single Drop Experiments}

5.4.2 Materials and Methodology for Multiple Drop Experimental System

\subsection{Summary}




\subsection{Introduction}

This chapter explains the development of a method for assessing the potential for the selective collection of fine particles. If successful, this system could then provide a means for selectively separating dry hydrophilic silica from dry hydrophobic coal. Preliminary experiments were conducted using single water drops to investigate the phenomena at a drop level (Section 5.2). Then a laboratory scale experimental system was designed and fabricated to test this concept at the multi-drop level. Design and development of the multiple drop experimental system is explained in Section 5.3. This explanation is followed by a description of the final experimental methodology and details of the materials used (Section 5.4).

\subsection{Preliminary Single Drop Experiments}

Preliminary single drop experiments were conducted using two different methods. The first method involved a vibratory table under a hanging water drop to energise the bed of particles as shown in Figure 5.1. In this case, the water drop hang over the particle bed with a distance of $30 \mathrm{~mm}$. The second method was conducted by sprinkling the particles, via a spatula, onto a hanging water drop. A surface tensiometer (OCA 20), which includes a needle support with vertical and horizontal fine adjustments, and a high speed video system was used to conduct these experiments. A single water drop hanging from the needle (I.D. $1.3 \mathrm{~mm}$ ) attached to a syringe was accommodated within the surface tensiometer system. Video microscopy was used to examine the content of the drops.

Preliminary single drop experiments were carried out with glass ballotini and coal particles in the 75 - $90 \mu \mathrm{m}$ size range, either separately or as a mixture. The main objective of these experiments was to determine the ability of a water drop to selectively capture the hydrophilic material. 

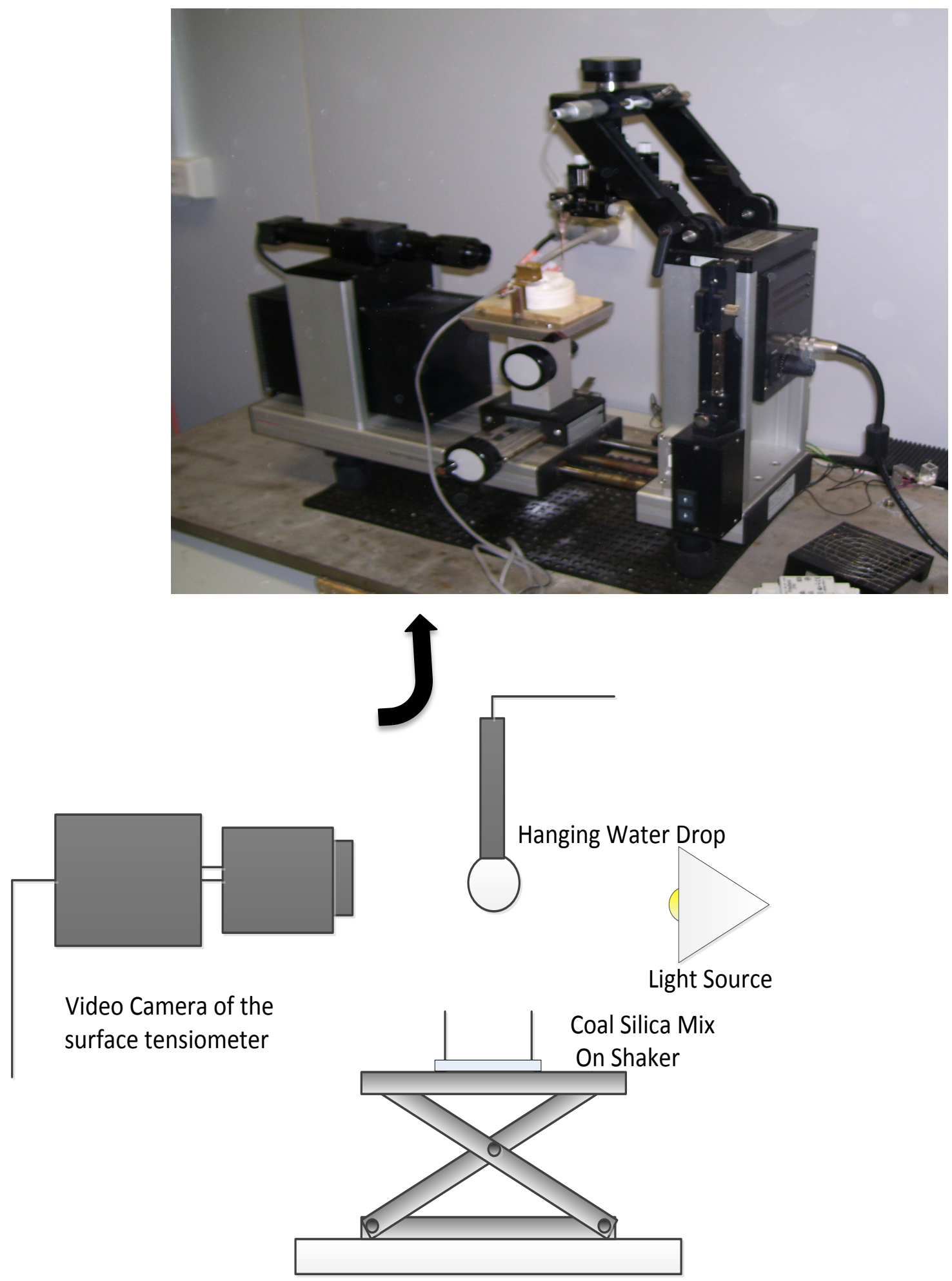

Figure 5.1: Single drop experimental set up, incorporating a vibratory support for energizing the fine particles located below the hanging drop. 


\subsection{Development of Multiple Drop Experimental System}

The aim of this apparatus was to develop a system for selectively collecting hydrophilic particles from an air suspension by using multiple falling water drops. There are several potential advantages of this approach compared with conventional flotation. Firstly, falling drops of water offer the potential for a higher surface flux than rising air bubbles, due to their higher terminal velocity. This potential was not explored in this study, however, with the water flux maintained at comparable levels to that used in flotation of order $0.01 \mathrm{~m} / \mathrm{s}$. Secondly, in principle the particle-droplet collision efficiency will be higher than between a particle and bubble, due to the much lower viscosity of the air film around a drop, compared to the liquid film around a bubble. This should be particularly advantageous for collecting fine particles, permitting a reduction in the necessary energy consumption. Thirdly, unlike the surfaces of gas bubbles, the surfaces of liquid drops permit the full engulfment of the hydrophilic particles, providing a capacity much higher than that offered by the interface.

In the case of fine coal processing, the natural hydrophobicity is amplified in the dry state (Nicol, 2001), and hence there should be no need for any chemical reagent. Moreover, the hydrophobic coal product should remain in a dry state, in order to achieve a higher calorific value product, without the need for dewatering through filtration.

The major challenge of this research was the development of a reliable laboratory scale device. This challenge required an iterative design approach, involving several laboratory scale prototypes. Figure 5.2 shows a basic schematic of the conceptual design. This system mainly consists of a feeding column, water distribution chamber mounted on a collision chamber, and an air suction pump. 


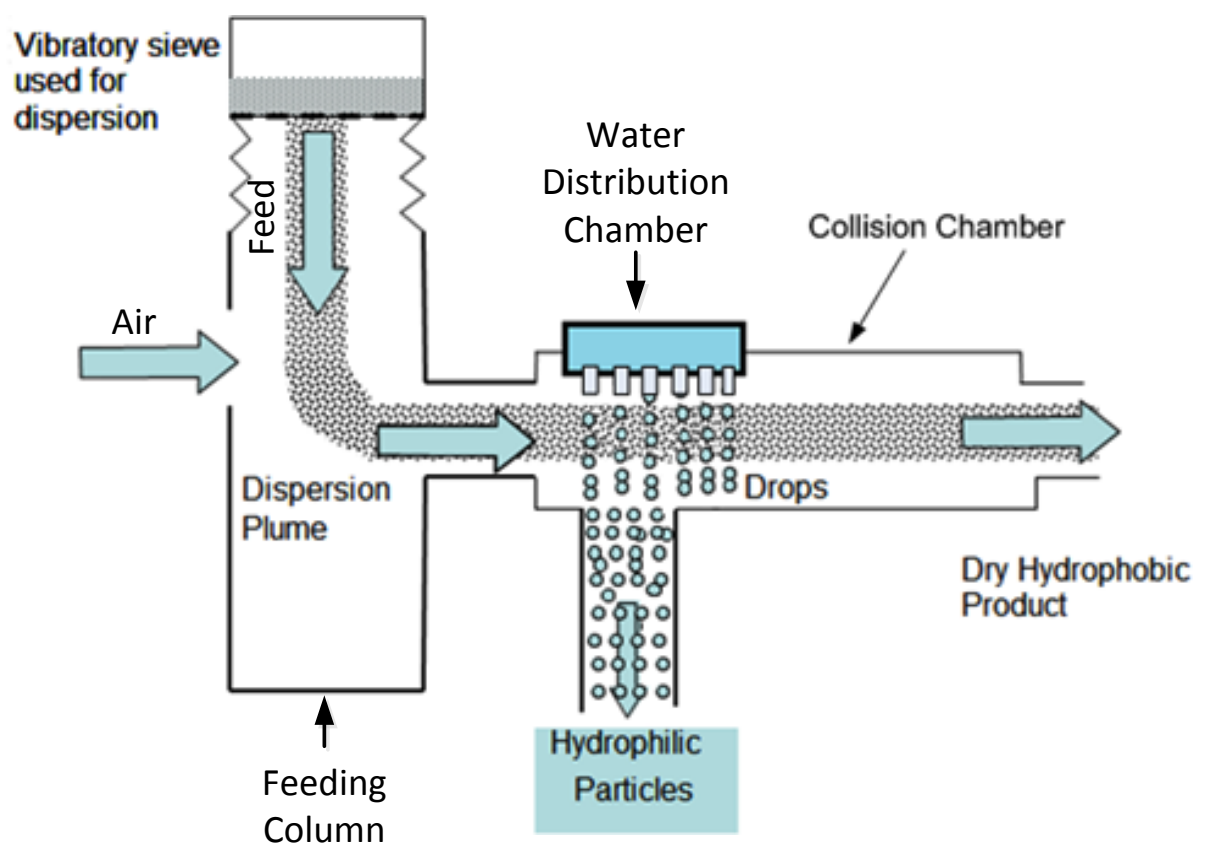

Figure 5.2: Schematic of the multi-drop experimental system.

The feed column suspends the dry particles in the air. This air suspension flows into the collision chamber where it interacts with the vertically falling water drops. The intention was that the hydrophilic silica would be selectively collected by the water drops, leaving much of the hydrophobic coal to exit via the dry air suspension. The coal particles were then separated from the air using a cyclone.

Development of a functional laboratory system based on this conceptual design required various modifications and additions before the required level of mixing and collision were obtained. The particulate feed was dispersed into the air using a vibrated sieve. The flow rate of solids on to this screen was controlled by an automated pair of valves as shown in Figure 5.3, which opened and closed alternately in order to minimise back flow of air. The air dispersed feed was projected into the collision zone horizontally while water drops were released vertically from the water distribution chamber above. This approach allowed the suspended particles to collide with the water drops. Water collected beneath the collision zone and drained into the underflow collection tank. Solids were removed from the gas stream using a cyclone. Air flow was generated by a vacuum suction pump located at the gas exit from the system and regulated by a valve at the gas inlet. A schematic of the initial prototype is shown in Figure 5.3. 


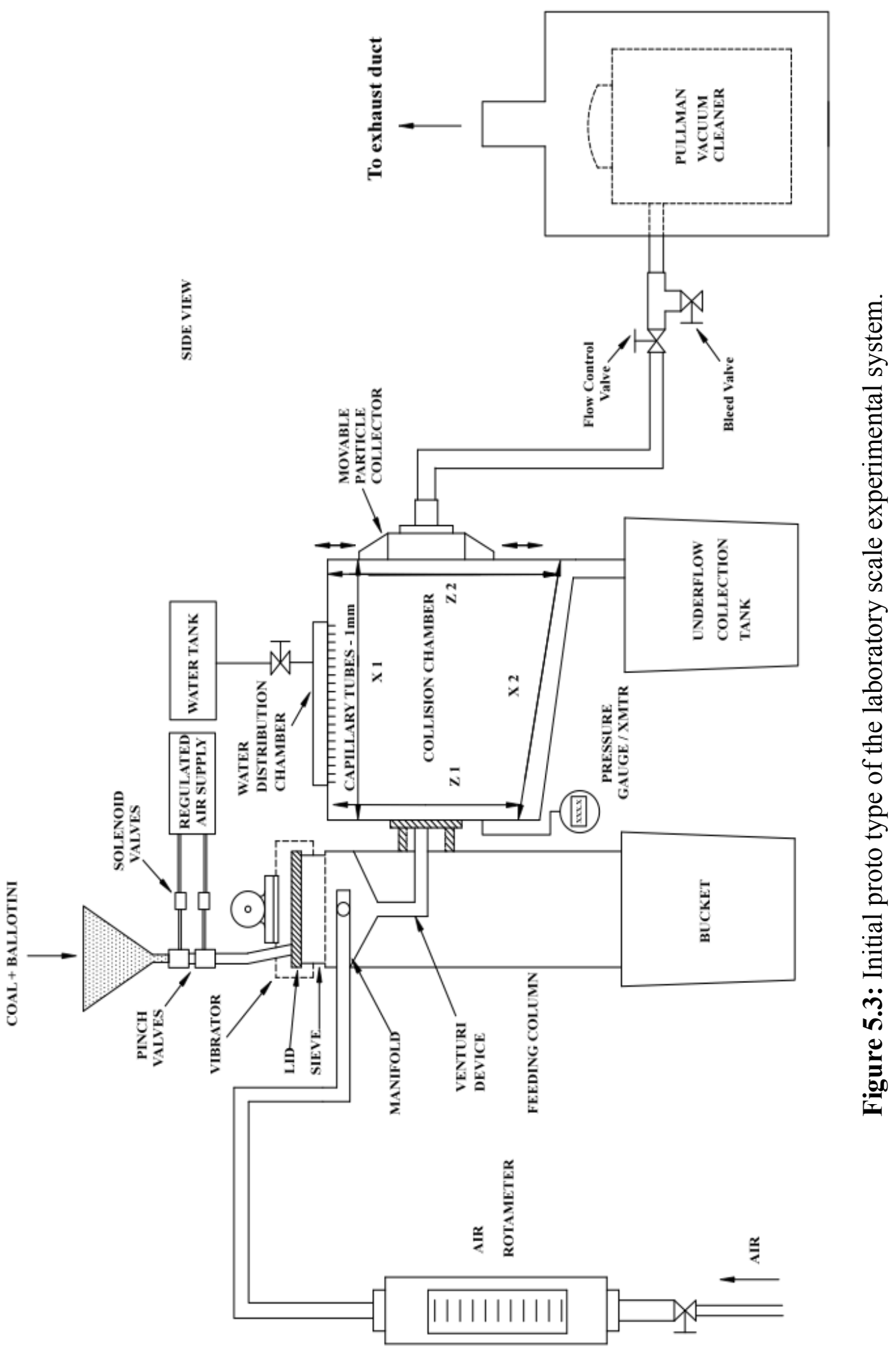




\subsubsection{Feeding Configuration}

As illustrated in Figure 5.3, the feed solids were introduced to the feeding column through a funnel. This feed was regulated by a pair of linch diameter pneumatically controlled pinch valves. The pinch valves were actuated using computer-controlled solenoids valves. These solenoid valves operate on $12 \mathrm{~V}$. The frequency of solenoid opening and closing was adjusted by programmed software to the solids feed rate.

The particulate solids were then dispersed in an air stream, which flowed into the chamber via a $180^{\circ}$ manifold, as shown in Figure 5.4. A 1.5 inch diameter air rotameter (flow meter) was used to measure the flow rate of air into the manifold. To evenly disperse solids a vibratory $150 \mu \mathrm{m}$ sieve was used. A venturi device was also used in this set up to convert this vertical plume to a horizontal plume to flow through the flotation chamber. Hence, the feed was carried through the flotation chamber by air as a gas dispersed plume. A small portion, which failed to flow into the system, was captured in a bucket at the base.

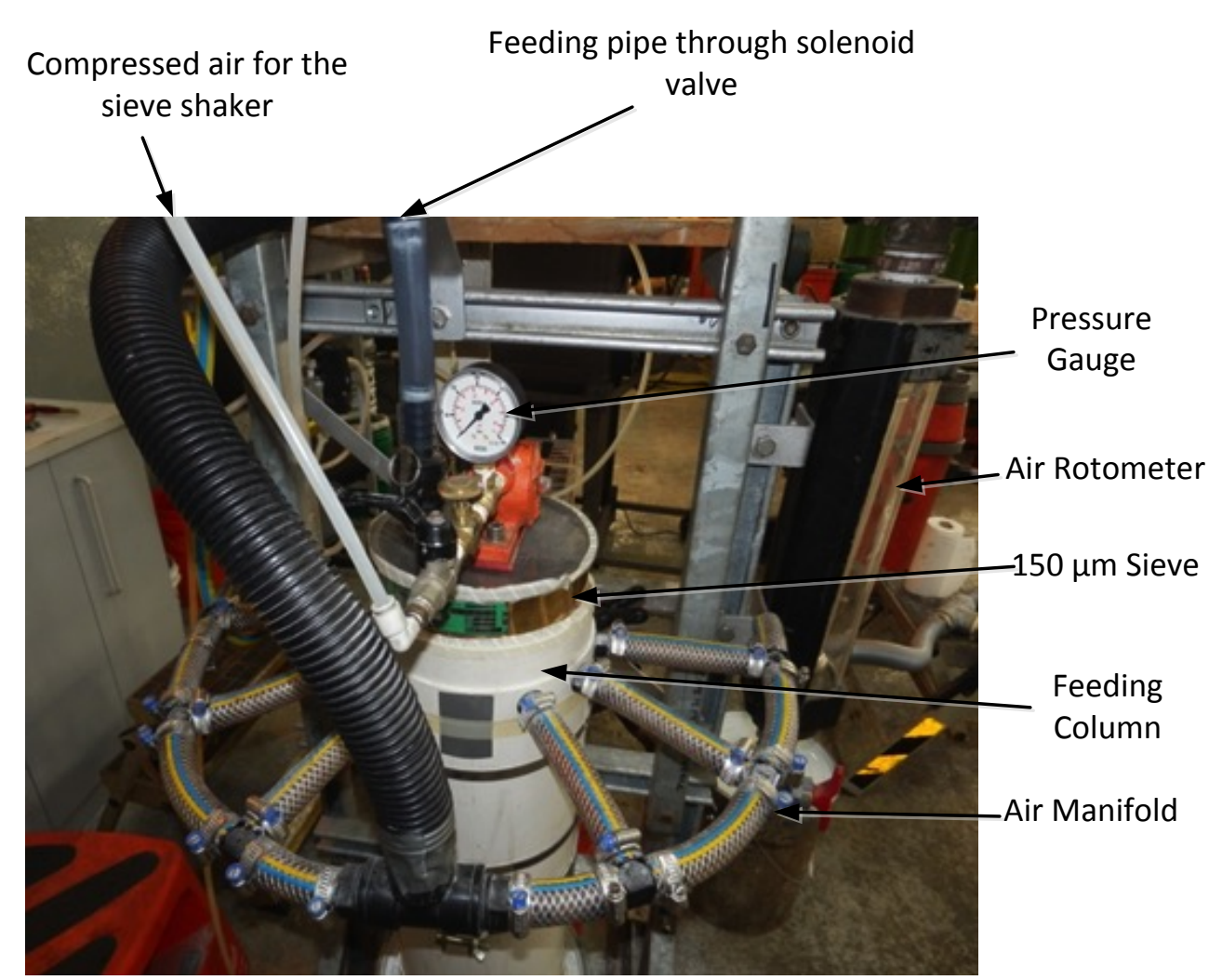

Figure 5.4: Air inlet manifold around the feeding column. 


\subsubsection{Collision Chamber and the Vacuum Pump}

As illustrated in Figure 5.3, the feeding column was attached to a Perspex collision chamber, with dimensions of $506 \mathrm{~cm}\left(X_{1}\right) \times 480 \mathrm{~cm}\left(X_{2}\right) \times 414 \mathrm{~cm}\left(Z_{1}\right) \times 515 \mathrm{~cm}\left(Z_{2}\right) \times$ $30 \mathrm{~cm}(Y)$ initially. The base of the flotation chamber had a $15^{\circ}$ fixed inclination to help insure the tailings drained under the influence of gravity.

The gas-suspended solids were projected at an optimum horizontal velocity, sufficient to insure that all of the particles would reach the exit on the other side of the chamber, with little or no particle fall out. This condition was tested in the absence of falling water drops. Thus, in the presence of falling water drops, it was possible to conclude that all particles reporting with the water were in fact captured through collision. The length of the collision chamber was also designed to be adjustable however only one length was used in this study. A $1000 \mathrm{~W}$ vacuum pump (Pullman AS 5 model) was used to generate the airflow through the collision chamber. This created a $5 \mathrm{kPa}$ vacuum inside the collision chamber.

\subsubsection{Water Distribution Chamber Design and Cyclone}

The water distribution chamber consisted of a closed rectangular cross-section shaped cell with dimensions of $17 \mathrm{~cm} \times 9 \mathrm{~cm} \times 4 \mathrm{~cm}$ as shown in Figure 5.5. There is a water inlet and an air removal outlet at the top of the water distribution chamber.

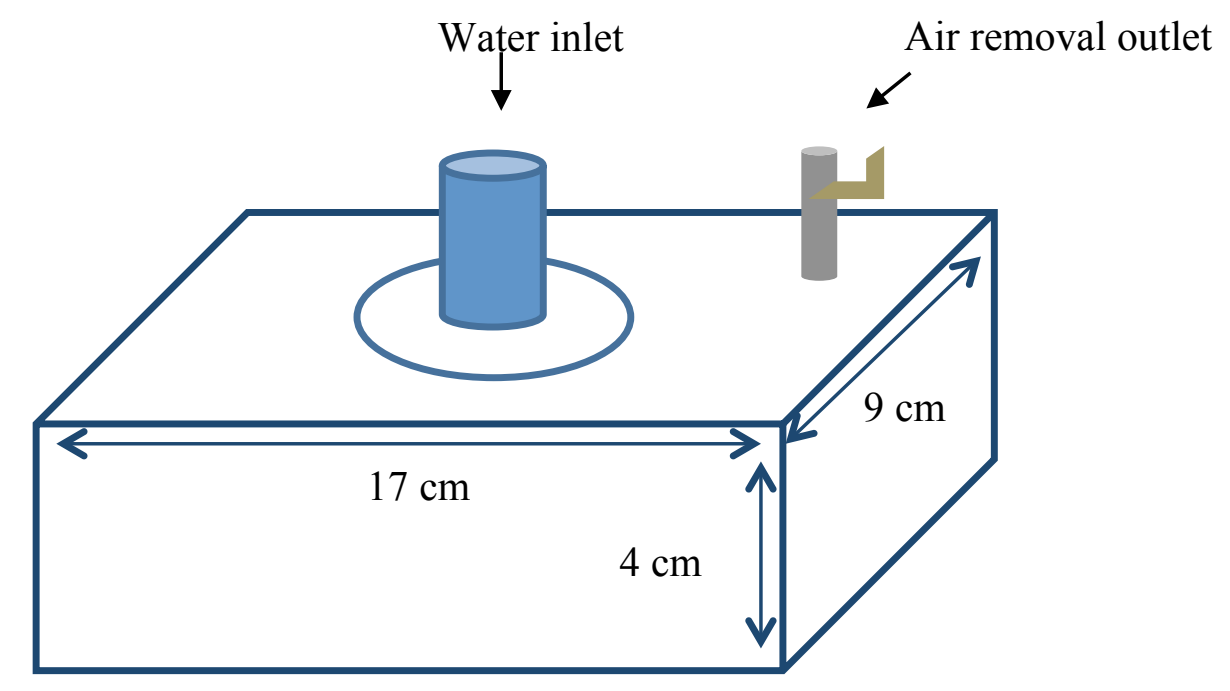

Figure 5.5: Design of water distribution chamber. 
The upper surface of the collision chamber consisted of an array of holes with nozzles, from which water drops exited. These nozzles were supplied by a water distribution chamber that was mounted on top of the collision chamber. The holes were aligned with stainless steel capillary tubes, of internal diameter of $0.8 \mathrm{~mm}$, protruding as a grid. This grid consisted of six rows across an area of $0.00198 \mathrm{~m}^{2}(2.46 \mathrm{~cm} \times 8.06 \mathrm{~cm})$ as shown in Figure 5.6. Each of these rows had a distance of $4 \mathrm{~mm}$ between the centres of the capillary tubes. Each of these capillary tubes produced drops with a maximum diameter of $3 \mathrm{~mm}$ (Refer to Appendix C for detailed calculations). A $12.5 \mathrm{~mm}$ diameter rotameter was used to measure the flow rate passing through the system of capillary tubes.

One of the design problems was the difficulty associated with transporting all of the particles across the collision chamber. To overcome this problem, two modifications were made to the design. The first modification involved a reduction in the height of the collision chamber to obtain a laminar air flow. This changed the shape of the cell to a square cross section with the dimensions of $60 \mathrm{~cm}(X) \times 11 \mathrm{~cm}(Y) \times 11 \mathrm{~cm}(Z)$. A schematic representation of the cross section of the modified chamber is included in Figure 5.6.

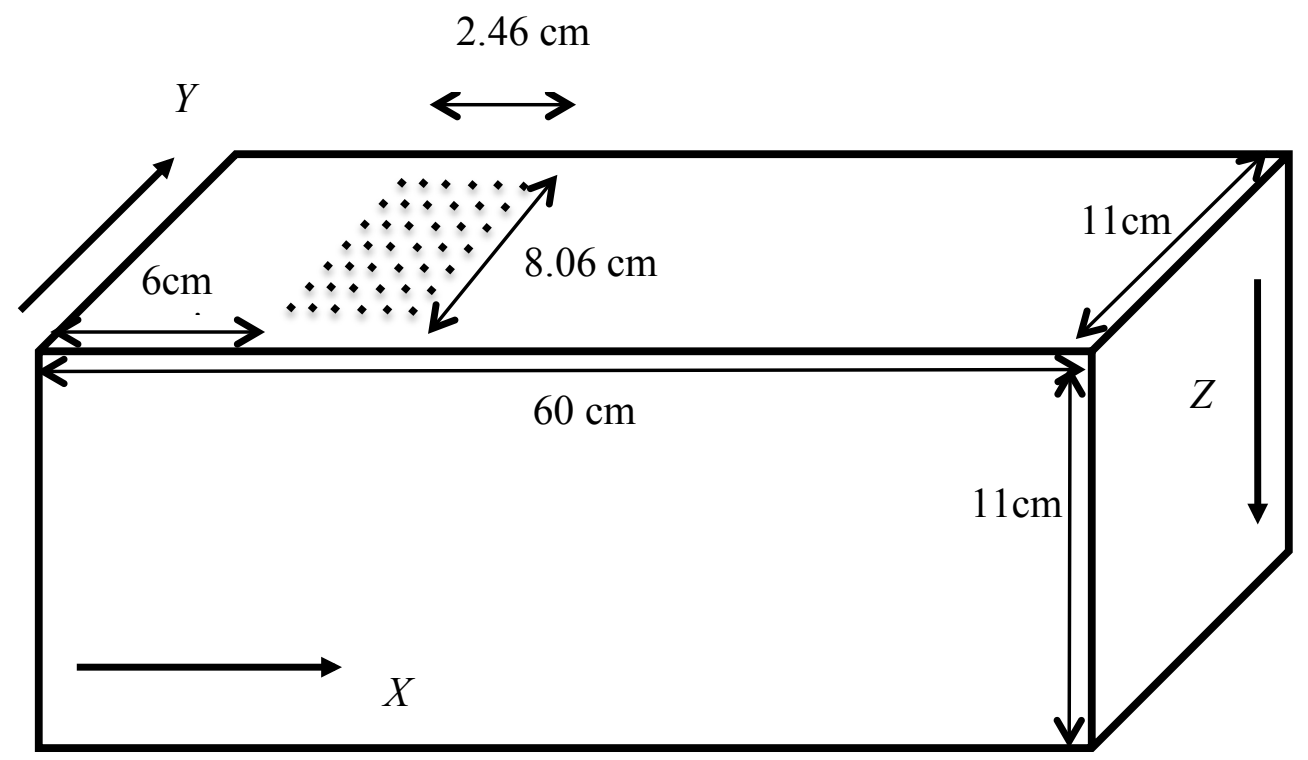

Figure 5.6: Modified collision chamber with water distributing capillary tubes. 
The second modification incorporated the addition of a flow straightner to the centre of the collision chamber. This device consisted of a metal grid which supressed the divergence of the air flow into the collision chamber by promoting laminar flow. A photograph of the flow straightener is shown in Figure 5.7.

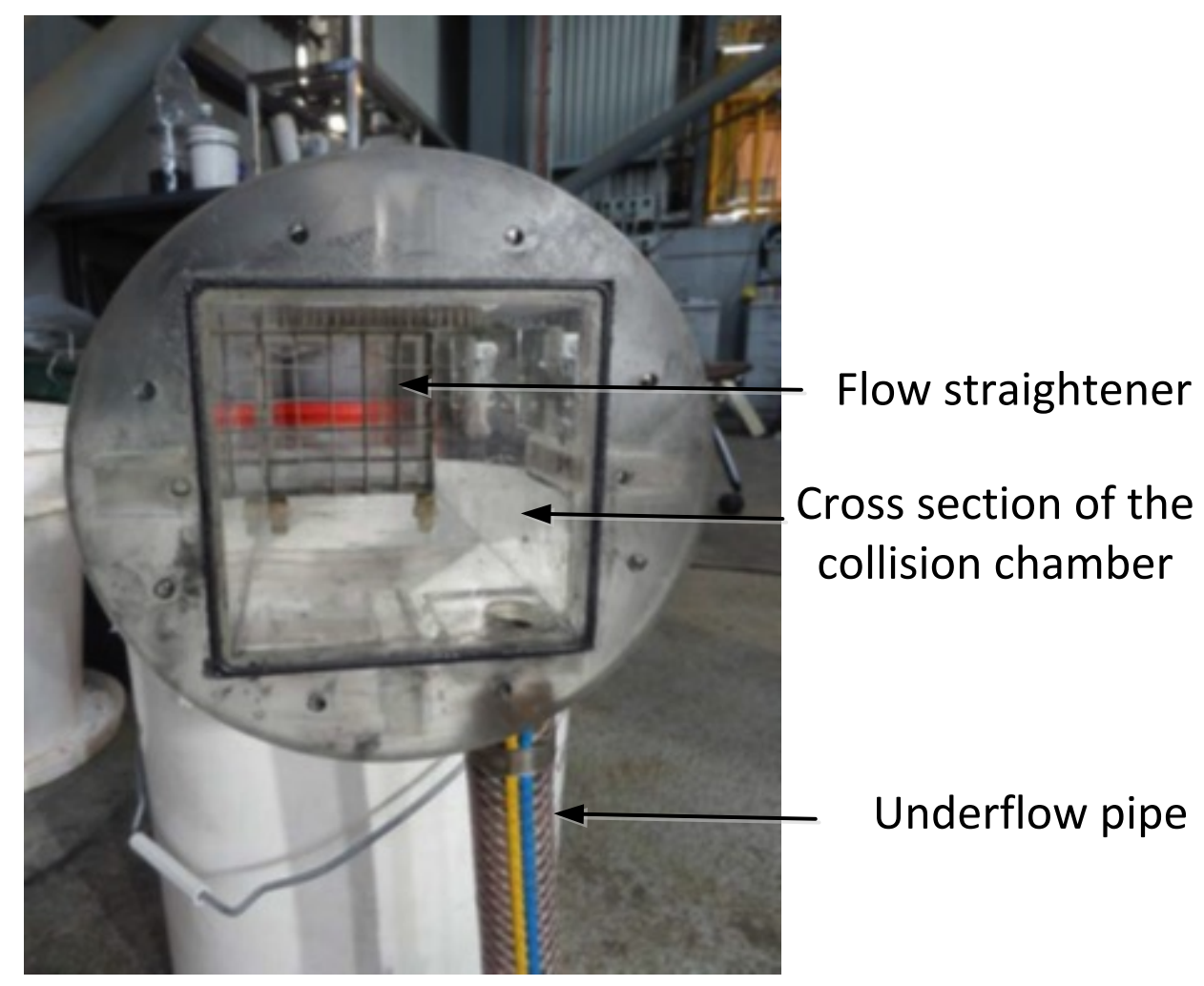

Figure 5.7 : Flow straightner visible at the rear of the image through which the air entered.

A low-pressure drop metal cyclone was designed and fabricated, and used to capture the fine particles after they emerged from the collision chamber. The fine particles dropped through the base of the cyclone and into a sealed container. A schematic representation of the final system is shown in Figure 5.8. Figure 5.9 provides a three dimensional (3D) representation of the same system, while Figure 5.10 provides a photographic image of the actual system. 


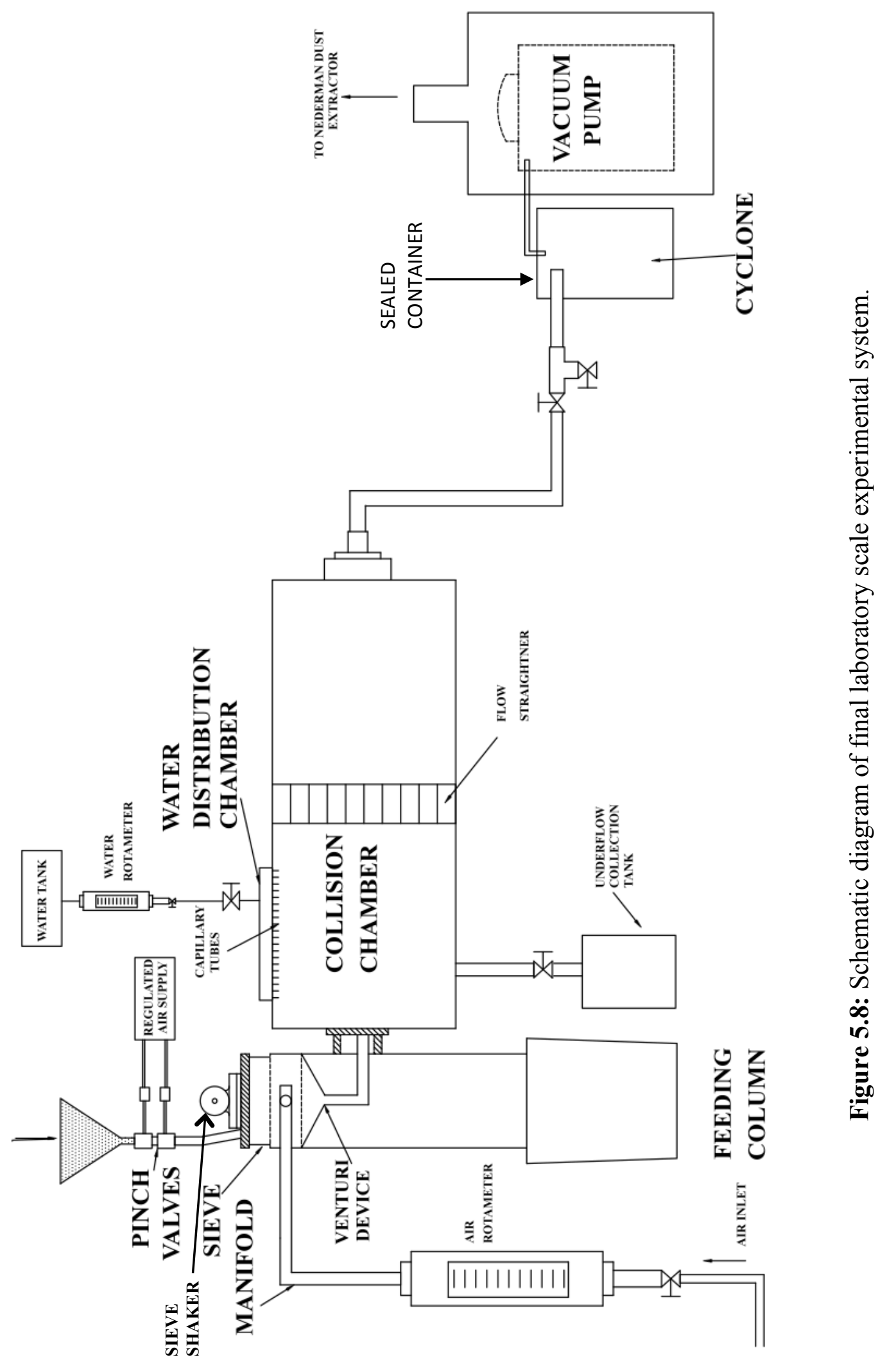




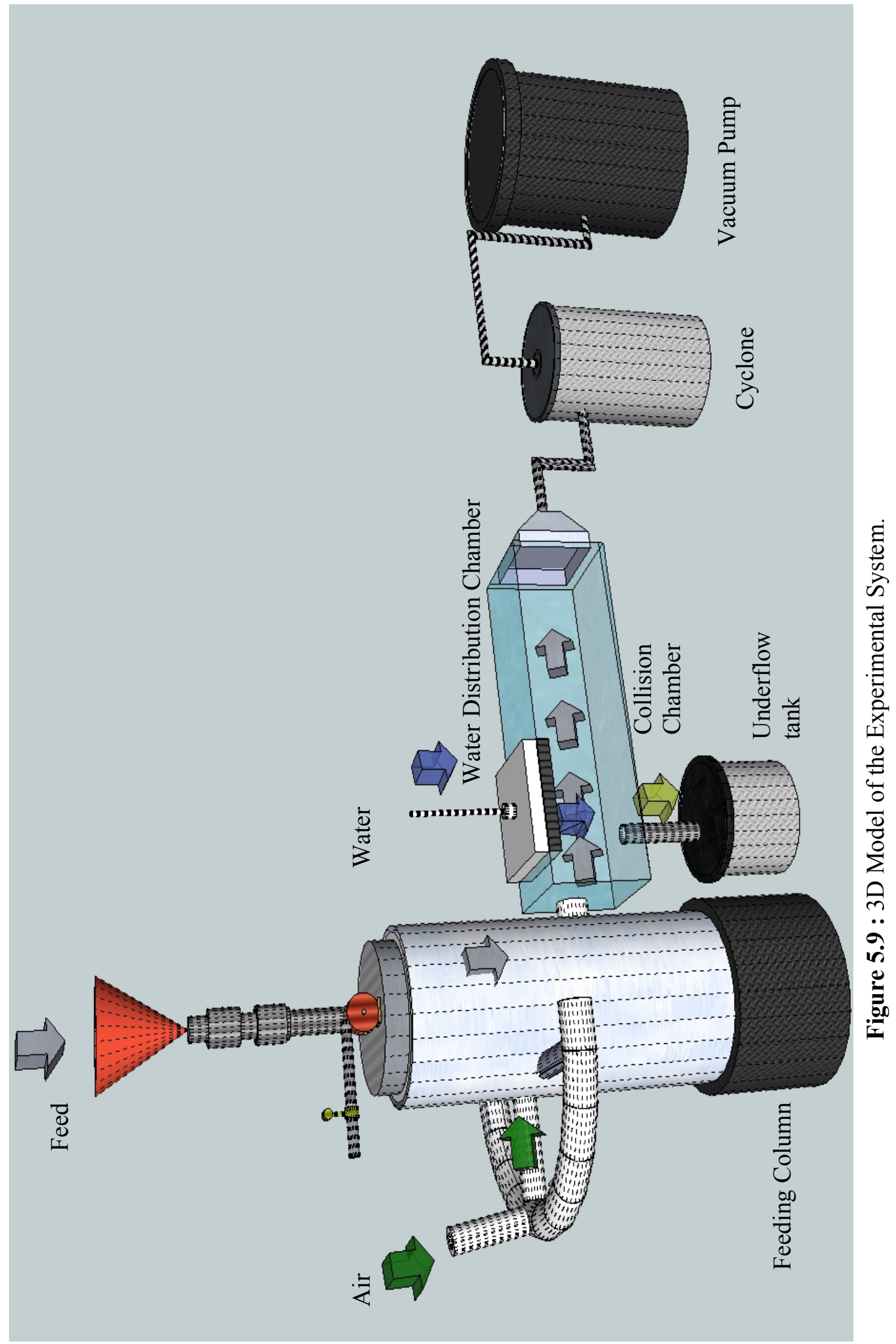




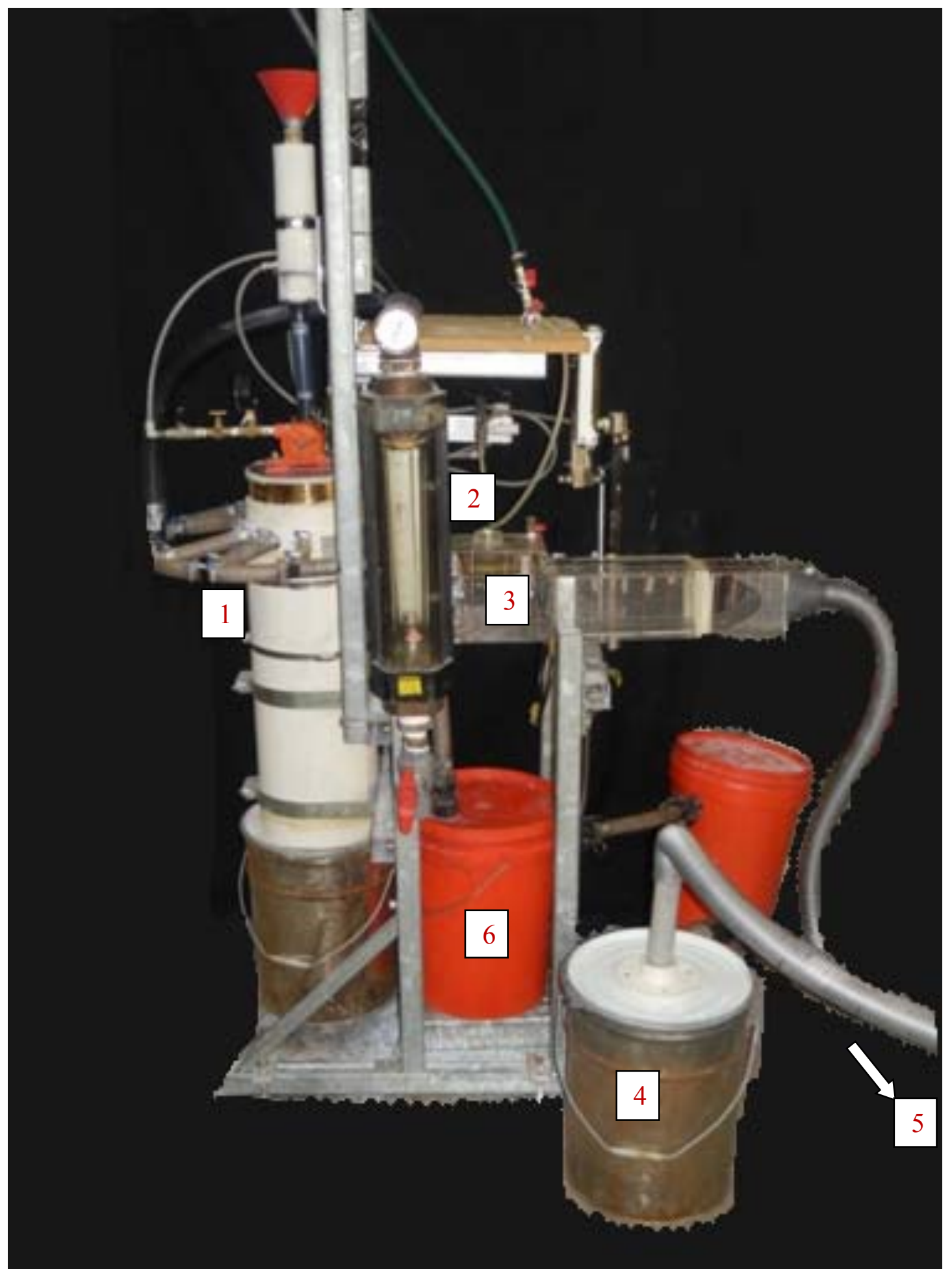

Figure 5.10: Photograph of the laboratory scale experimental system

(1) Feeding column (2) Water distribution chamber (3) Collision chamber

(4) Cyclone (5) Tube connecting to vacuum pump (6) Collecting tanks. 


\subsection{Experimental Methodology and Materials}

\subsubsection{Methodology and Materials for Single Drop Experiments}

Particles of ballotini were dry sieved using 75 and $90 \mu \mathrm{m}$ sieves to obtain the fine particles used in the single drop experiments. Coal samples in the same size range were also prepared, but this time using wet sieving, as dry sieved coal samples still contained a large amount of very fine particles (less than $75 \mu \mathrm{m}$ ).

\subsubsection{Materials and Methodology for Multiple Drop Experimental System}

Several particle species were used in the experimental program, each separated into specific size fractions. Table 5.1 provides a summary of the data as measured using a Malvern Mastersizer 2000. Appendix A provides full details of the particle size distributions of the particles. Because of the inefficiencies in fine particle screening most of the particle size ranges have median values $\left(d_{50}\right)$ well below the nominal values.

Table 5.1: Malvern Mastersizer 2000 size measurements of the different material types used in the multiple drop experiments.

\begin{tabular}{lllll}
\hline Material & $\begin{array}{l}\text { Nominal Size } \\
\text { Range }(\mu \mathrm{m})\end{array}$ & $\begin{array}{l}d_{10} \\
(\mu \mathrm{m})\end{array}$ & $\begin{array}{l}d_{50} \\
(\mu \mathrm{m})\end{array}$ & $\begin{array}{l}d_{90} \\
(\mu \mathrm{m})\end{array}$ \\
\hline Glass ballotini & $0-38$ & 17 & 25 & 36 \\
& $38-45$ & 23 & 33 & 47 \\
\hline Silica flour & $0-22$ & 4 & 15 & 37 \\
& $10-20$ & 8 & 16 & 29 \\
& $20-40$ & 16 & 27 & 42 \\
& $38-45$ & 21 & 34 & 54 \\
\hline \multirow{2}{*}{ Coal } & $38-45$ & 3 & 14 & 38 \\
& $38-90$ & 29 & 64 & 105 \\
& $45-63$ & 30 & 52 & 78 \\
& $75-90$ & 24 & 77 & 116
\end{tabular}

The glass ballotini (Grade AI, supplied from GB11, Potters Pty. Ltd.) had a quoted nominal size range of minus $53 \mu \mathrm{m}$ and specified density of $2600 \mathrm{~kg} / \mathrm{m}^{3}$. This ballotini 
was classified using a $24 \times 1.7 \mathrm{~mm}$ channel Reflux Classifier with a channel velocity of $0.01943 \mathrm{~m} / \mathrm{s}$. The overflow fines were then dried and screened to produce two different fractions with size ranges $23-47 \mu \mathrm{m}$ and $17-36 \mu \mathrm{m}$. Galvin et al. (2010) provides more details about the Reflux Classifier and the method used.

Rough silica flour (Potters, Grade 400G), which had a density of $2600 \mathrm{~kg} / \mathrm{m}^{3}$, was classified by wet screening using filter bags to produce a $4-37 \mu \mathrm{m}$ size fraction. The $21-54 \mu \mathrm{m}$ size fraction was produced by dry screening. A Queensland coking coal (Mole Vale coal) with $6 \%$ ash content was wet screened to make different size range samples. Four different size fractions were prepared, as shown in Table 5.1.

\section{(a) Surface Modification through Cleaning}

Representative $50 \mathrm{~g}$ samples of all fractions of glass ballotini and silica flour were treated separately with 10\% w/w Extran ${ }^{\circledR}$ MA 03 (phosphate) dissolved Milli-Q water. These samples were sonicated for 20 minutes to disperse properly and soaked overnight. This preparation was followed by rinsing with Milli-Q water four times before drying overnight in an oven at $100{ }^{\circ} \mathrm{C}$. The dried samples were then stored in an air tight desiccator before being used in each experiment. These samples were redried at $70{ }^{\circ} \mathrm{C}$ for one hour before preparing $10 \mathrm{~g}$ air sealed packs for every experiment. The purpose of heating the sample was to remove moisture from the particles. These samples were allowed to cool down on the bench top before using in experiments.

\section{(b) Surface Modification through Esterification}

Glass ballotini in the $38-45 \mu \mathrm{m}$ size range were hydrophobised through an esterification reaction with straight long chain alcohols. This esterification reaction creates a covalently bonded alkane coating around glass ballotini to make them hydrophobic. $50 \mathrm{~g}$ of surface cleaned ballotini were mixed with $130 \mathrm{~g}$ of 1-dodecanol in a conical flask. Then this conical flask was insulated and boiled, facilitating reflux condensing for $7 \mathrm{~h}$ with continuous magnetic stirring. After $7 \mathrm{~h}$ of reaction, particles were decanted from the alcohol and washed three times with acetone. This acetone washing was followed by ethanol washing (twice) and final washing with Milli-Q water. 


\section{(c) Salt Solution Preparation}

The salt solutions were made using aluminium sulphate (commercial name Purex Pool Floc) supplied by Price Chemicals. This salt was chosen because of the high valency of the ions. High valency is desired because it will have a large effect on surface charges. This salt was dissolved in tap water to create solutions with concentrations up to 2000 $\mathrm{mg} \cdot \mathrm{L}^{-1}$ and with conductivities up to $920 \mu \mathrm{S}$.

\section{(d) Operating Procedure of the Multi-Drop Experimental System}

After connecting all the equipment, the vacuum cleaner was turned onto suck air through the system and the flow rate adjusted to the desired level. Then the valve on top of water chamber was opened to start the cascade of falling water drops. Then the solids feed was loaded into the feed funnel. The software controlling the two feed pinch valves was set to open and close, as required, to achieve the desired solids feed rate.

With a constant airflow rate through the rotameter, $50 \mathrm{~g}$ of each sample was fed to the system in $10 \mathrm{~g}$ packs, and the total mass recovered in the underflow was collected. During most of the experiments, $10 \mathrm{~g}$ of particles were fed within $10 \mathrm{~s}$. These particles were projected in to the collision chamber through a pipe with a diameter of $4 \mathrm{~cm}$ as shown in Figure 5.14. These packets were fed onto the screen within approximately 15 $\mathrm{s}$ intervals. This optimum concentration rate was determined after considering recovery of several trials. Most experiments were stopped within 2 min of the last packet of feed. However, some materials, such as coal $(<38 \mu \mathrm{m})$ and very fine silica $(<21 \mu \mathrm{m})$, were cohesive and often did not fully pass through the sieve, even after an extended time of up to 5 min vibrated on the screen.

After the solids feeder was switched off, the airflow was switched off, followed by the water flow. Any solids residue left in the first half of the collision chamber were also collected and considered part of the underflow. The particles in the underflow were allowed to settle and the excess water decanted. Then the particles were dried overnight in an oven at $100{ }^{\circ} \mathrm{C}$ and weighed. These particles were termed the underflow. 
Particles that were not carried away in the underflow with the water drops were instead collected in the cyclone. These dry solids were also collected and weighed. Material left on the flow straightener and in the second half of the collision chamber was washed out, collected, dried and included as part of the overflow.

This procedure was then usually repeated for four or five different water flow rates, with duplicate experiments performed as required if the experimental losses were too high (more than $15 \% \mathrm{w} / \mathrm{w}$ ). The recovered mass, as a percentage of the total feed, was recorded along with the water flow rate.

The five different water flow rates used are given in Table 4.1(Chapter 4) with respect to their volumetric rates $(\mathrm{L} / \mathrm{min})$ and superficial velocities $(\mathrm{m} / \mathrm{s})$ through the cross section of collision chamber. The water rotameter was calibrated before the readings were taken and the plot of this is given in Appendix B. The superficial velocity (volumetric flux) of the water was calculated by dividing the volumetric flow rate by the nominal flow area of the array $(2.46 \mathrm{~cm} \times 8.06 \mathrm{~cm})$. The average diameter of the water drops was $3 \mathrm{~mm}$ as per the calculations in Appendix C. The diameter of the drop did not vary with the water flow rate. These drops fell with a velocity of $1.04 \mathrm{~m} / \mathrm{s}$ in the collision zone (Eq 4.19, page 87).

A constant airflow rate of 50.1 Normal cubic meters per hour $\left(\mathrm{Nm}^{3} / \mathrm{h}\right.$ - normal cubic meter is the metric expression of gas volume at standard conditions and it is usually defined as being measured at $0{ }^{\circ} \mathrm{C}$ and 1 atmosphere of pressure) through the rotameter was used in each of the experiments, a velocity sufficient to ensure all of the particles could reach the cyclone in the absence of the falling drops. Appendix D shows the percentage of recovery of different particle types in the absence of water. Given the chamber cross section was $11 \mathrm{~cm} \times 11 \mathrm{~cm}$, this results a nominal air velocity of 1.39 $\mathrm{m} / \mathrm{s}$. Velocicalc ${ }^{\circledR}$, air velocity meter (Model: 9535A, TSI Incorporated, USA) readings were taken at three points along the centre line. Measurement results are shown in Figure 5.11. Velocicalc ${ }^{\circledR}$ measures the resultant airflow velocity in $\mathrm{m} / \mathrm{s}$ at any given point. Additionally, an experiment was carried out in order to measure the variation of the airflow velocity at points that are perpendicular to the horizontal centre line at various horizontal distances along the latter. Airflow velocities at points that lie on three 
vertical planes as shown in Figure 5.12 are measured using Velocicalc ${ }^{\circledR}$ meter. The airflow velocity measurements at these points are shown in Figure 5.13.

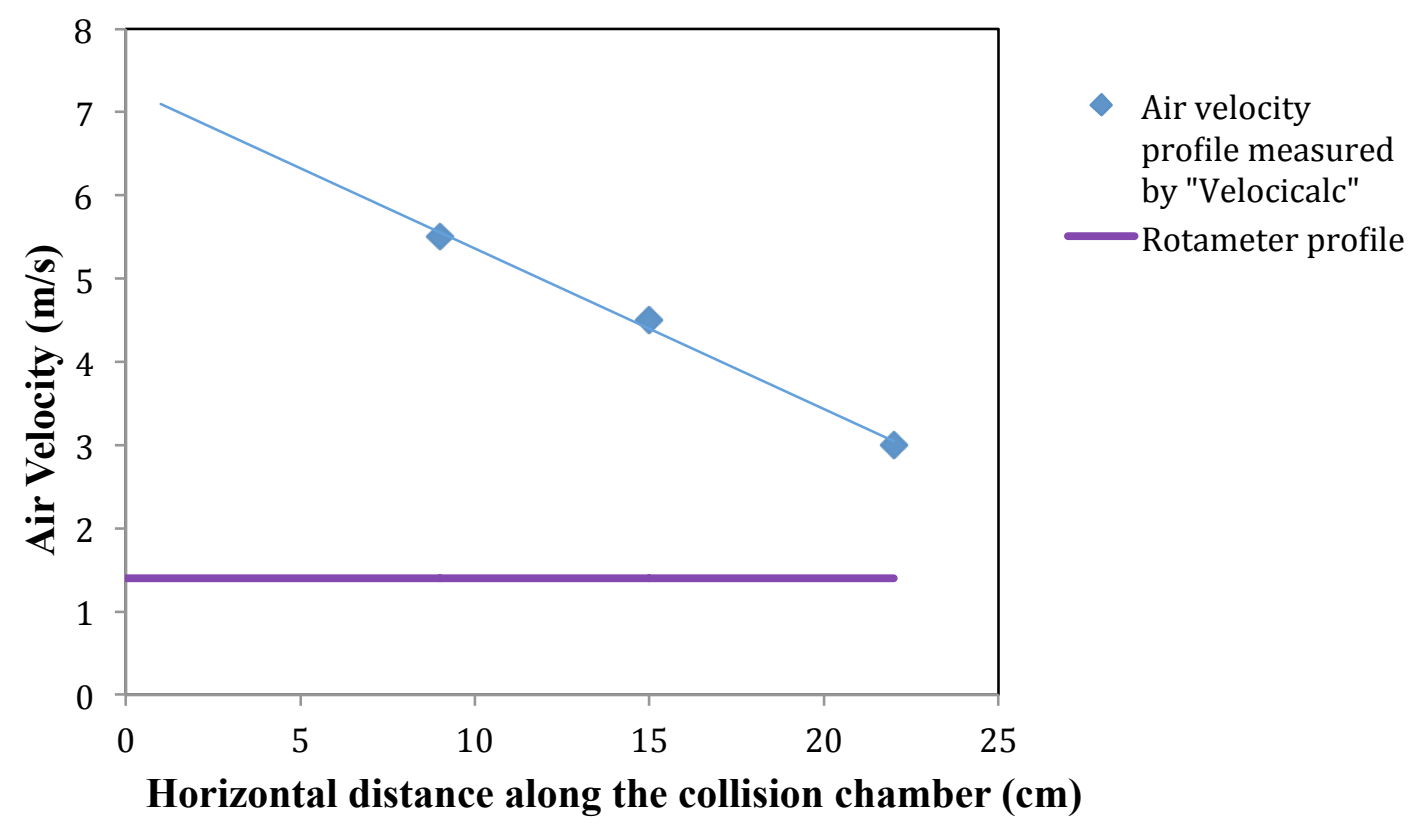

Figure 5.11: Velocity profiles of the airflow along the centre line of the collision chamber measured by Velocicalc ${ }^{\circledR}$ and using an air rotameter.

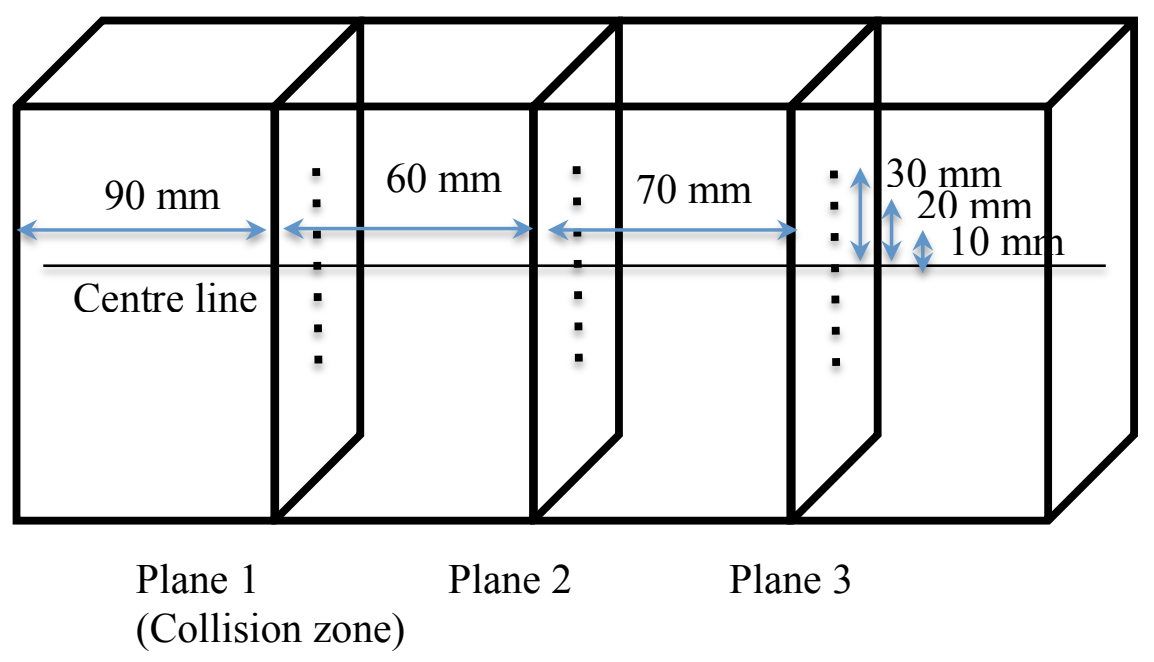

Figure 5.12: Measurement points for horizontal air velocity profiles. 


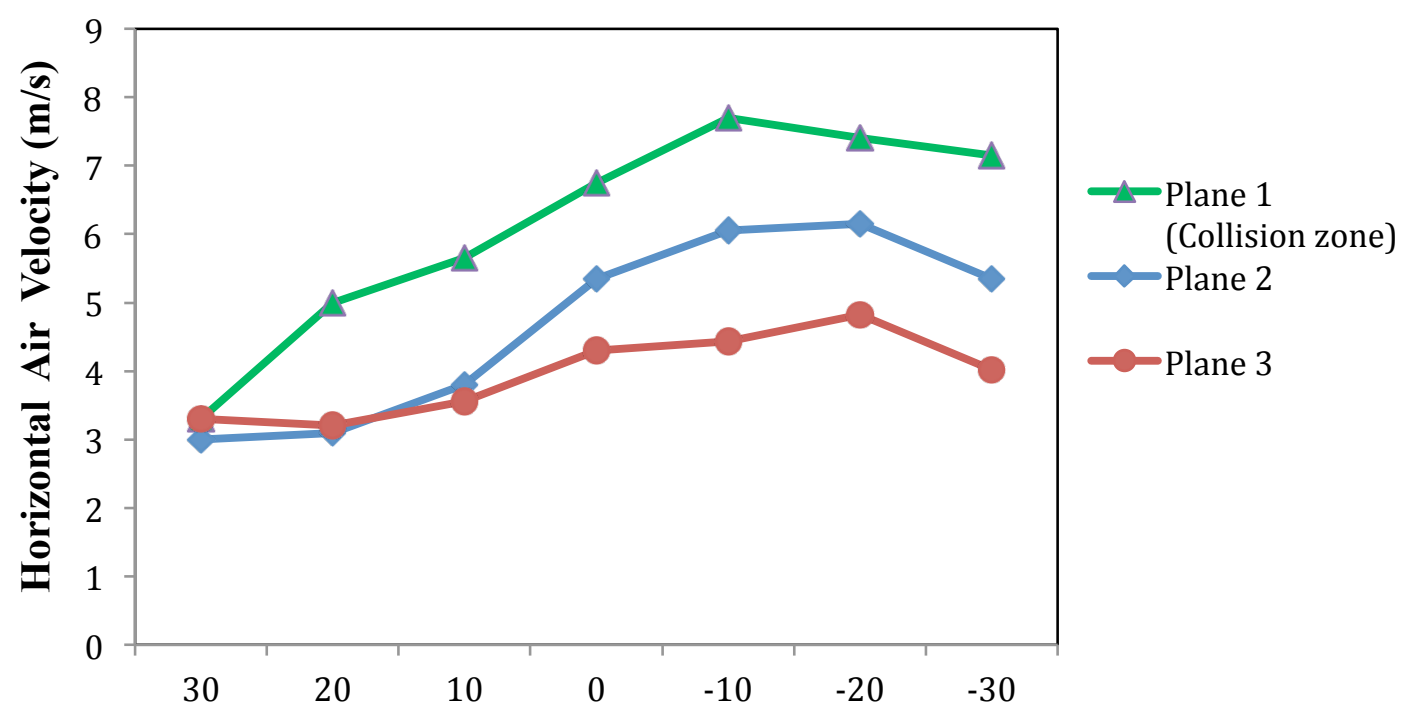

Distance from the centerline of the chamber $(\mathrm{mm})$

Figure 5.13: Horizontal airflow velocity variation at different heights within the collision chamber, measured for three horizontally distributed planes.

The peak velocities for all three planes (Figure 5.13) are recorded for points that are located below the centre line. For example, the maximum velocity for Plane 1 is recorded for a point that is located at $10 \mathrm{~mm}$ below the centre line. This can be explained from the parabolic path of the particle air mixture within the collision chamber. The particle/air mixture that enters the collision chamber forms a plume with a finite cross section that follows a parabolic path due to the effect of gravity on particles. Particles that are projected with a slightly upward entering velocity travels above the centre line with a deceleration resulting in low velocity measurements for points that lie above the centre line. Particles that enter either horizontally or with a slightly downward entering velocity tend to have higher velocities below the centre line as the horizontal distance along the centre line increases.

The typical air velocity in the vicinity of the falling drops was measured to be $6 \mathrm{~m} / \mathrm{s}$, hence the particle velocities were also assumed to be $6 \mathrm{~m} / \mathrm{s}$, which is higher than the drop falling velocity of $1.04 \mathrm{~m} / \mathrm{s}$. This velocity is also much higher than the theoretical average superficial velocity of $1.39 \mathrm{~m} / \mathrm{s}$ shown in Figure 5.11 . Superficial velocity is calculated by assuming that the airflow occurs through the whole cross section $(11 \times 11$ 
$\mathrm{cm}^{2}$ as shown in Fig. 5.6) of the collision chamber. It also assumes that the air pressure inside the collision chamber is evenly distributed. However, the actual flow of the particle/air mixture, which is projected through a pipe with a diameter of $6 \mathrm{~cm}$ as shown in Figure 5.14, does not evenly distribute through the whole cross section of the collision chamber. This results in a lower cross sectional area for the flow of the particle/air mixture; hence the locally measured velocity is higher than the calculated superficial velocity. However down stream the localised velocity was found to be around $3 \mathrm{~m} / \mathrm{s}$.

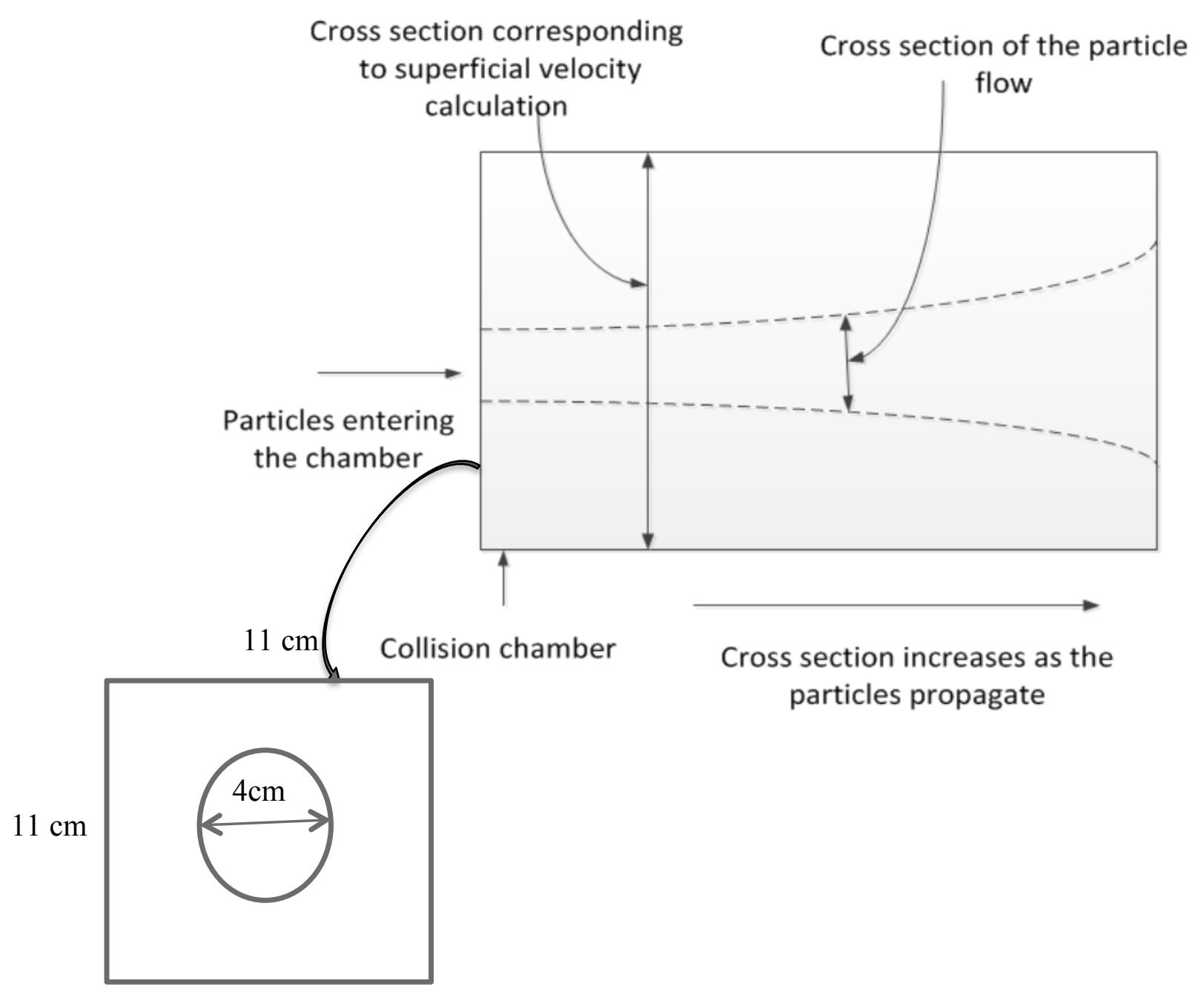

Figure 5.14: Velocity profile of the particle-air plume inside the collision chamber and the cross section of the projecting pipe. 
In all of the experiments, normal tap water was used. The $\mathrm{pH}$ of this water was 6.94 and the conductivity was $240 \mu \mathrm{S}$. All experiments were carried out at room temperature (20 $\left.\pm 4{ }^{\circ} \mathrm{C}\right)$.

\subsection{Summary}

A novel experimental system was designed and developed to investigate the selectivity of the collisions between particles dispersed within a gas and multiple falling water drops. This system allows the particles in a gaseous dispersion to interact with water drops from the gas side of the gas-liquid interface instead of the liquid side of the gasliquid interface as in conventional flotation. This approach should result in improved kinetics and recovery of fine particles due to the very low gas viscosity.

Preliminary experiments were conducted using single water drops to observe the particle engulfment phenomena at a single drop level. The development of a laboratory experimental system involved some major challenges, such as maximising the collision efficiency with dry fine particles with falling drops, obtaining a constant particle flux in the collision zone with finer particles and obtaining a dry product after the separation. Several prototype designs were required in order to achieve a robust result.

The multi-drop system consisted of three main sections: a feeding column, a collision chamber and a water distribution chamber. This design provided a large collision area per unit volume by using small falling water drops. Particles were introduced to the collision chamber with a nominal constant airflow velocity of $1.39 \mathrm{~m} / \mathrm{s}$ while drops fell with a velocity of $1.04 \mathrm{~m} / \mathrm{s}$ in the collision zone in all of the experiments. This rate was selected based on the minimum level necessary to carry all of the particles across the collision chamber when no falling drops were present. 


\section{Chapter 6}

\section{RESULTS AND DISCUSSION}

\subsection{Introduction}

\subsection{Results of the Preliminary Single Drop Experiments}

\subsection{Results of the Multiple Drop Experimental System}

6.3.1 Recovery Variation with the Water Flux

6.3.2 Comparison of Experimental, Analytical and DEM Results

6.3.3 Effect of Surface Modification

6.3.4 Recovery Variation with Different Size Particles

6.3.5 Effect of Particle Size and Shape

6.3.6 Effect of Different Size Hydrophobic Particles

6.3.7 Effect of Different Particle Types

6.3.8 Effect of Air Flow Rates

6.3.9 Recovery Variation with the Water Quality Changes

6.3.10 Separation of Coal-Silica Particle Mixtures

\subsection{Discussion}

\subsection{Conclusions}




\subsection{Introduction}

Experimental observations and the results obtained during the investigation of the novel system discussed in Chapter 5 are presented and discussed in detail in this chapter. This chapter begins with an outline of the basic observations for the preliminary single drop experiments, and then describes the experimental results obtained using the multipledrop experimental facility. Results collected from the laboratory experimental system were studied under various conditions. These conditions covered different water fluxes, particle species, particle sizes, air flow rates, and water quality. Challenges associated with the processing of ultrafine particles are also addressed.

\subsection{Results of Preliminary Single Drop Experiments}

Preliminary single drop experiments were conducted using two different methods as noted in Chapter 5.2. Initially, only samples containing one particle species was examined using the vibratory system. Secondly, a mixture of the coal and silica particles was vibrated under the hanging water drop. When a particle sample containing only glass ballotini particles was vibrated, the particles were rapidly engulfed by the water drop. After some point glass ballotini particles did not engulf in to the water drop, instead remained outside surface of the drop. Figure 6.1(a) has captured when the drop is saturated with the engulfed ballotini. When coal particles were vibrated, the particles attached to the outer surface of the water drop as shown in Figure 6.1(b). After an extended period of vibration, coal particles saturated the outer surface of the drop. In this case, there was no engulfment. Teflon particles showed a similar behaviour to coal particles. An optical microscope was used to study the effects of much more dilute conditions. Figure 6.2 shows spherical glass ballotini particles inside the water obtained from one experiment. 


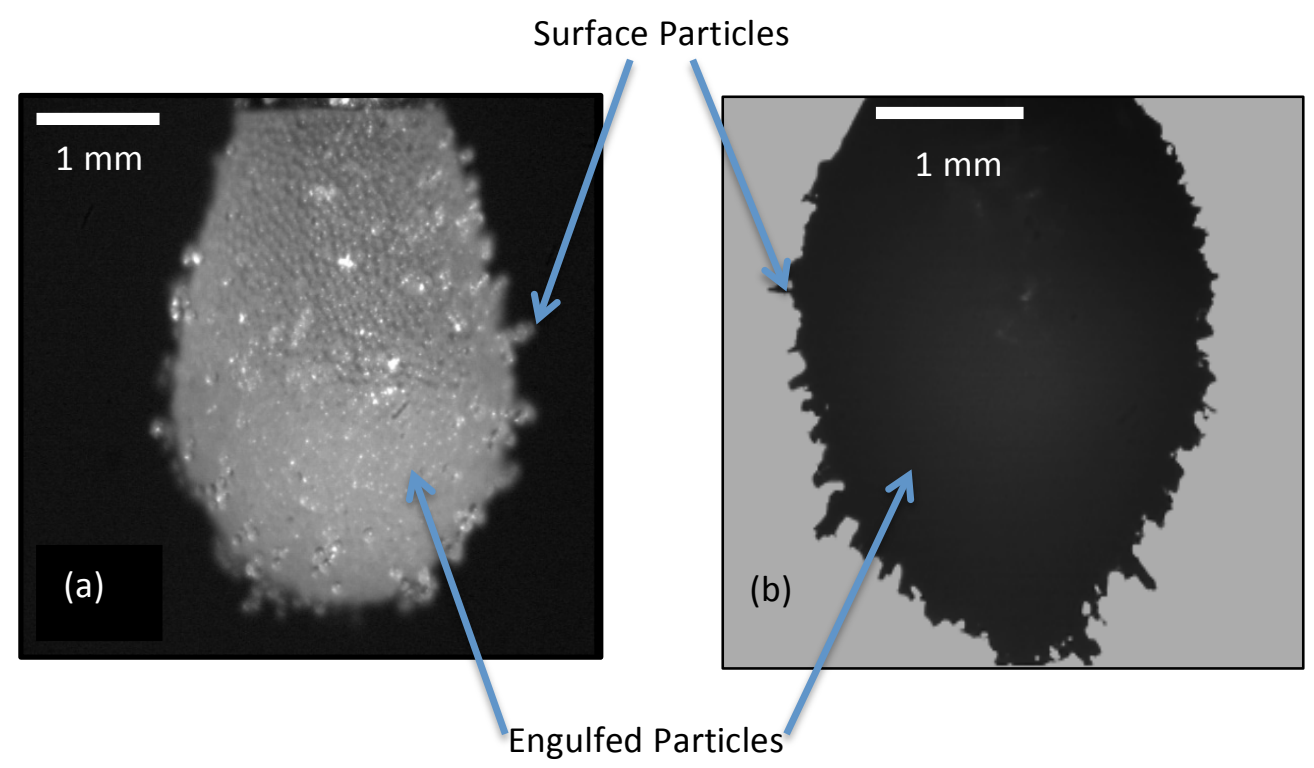

Figure 6.1: Photographs of pendent water drops after exposure to (a) glass ballotini and (b) coal particles. In both cases, pendent drops of water were saturated by the particles used.

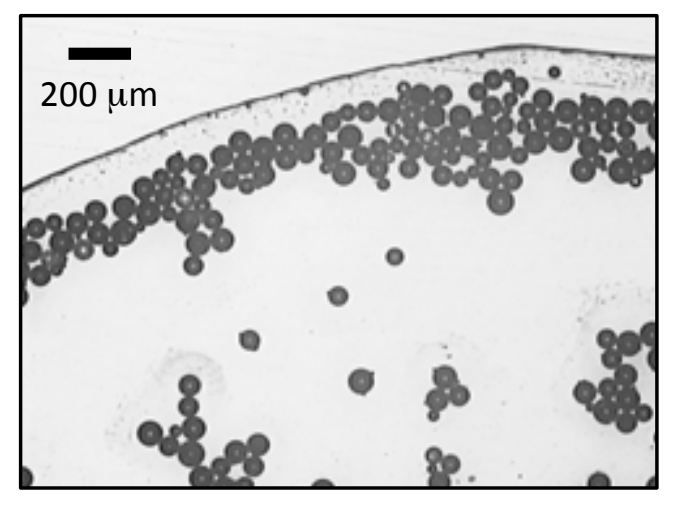

Figure 6.2: Microscopic image of water drop showing spherical glass ballotini. This shows the spherical glass ballotini particles inside the drop.

When the mixture of ballotini and coal was vibrated, a considerable amount of ballotini particles were observed to be inside the water drop. There was also evidence of fine coal at the drop surface as shown in Figure 6.3 (a). Similar results were observed when the particles were deposited from above, as shown in Figure 6.3(b). Experiments were repeated to ensure the findings were consistent. 


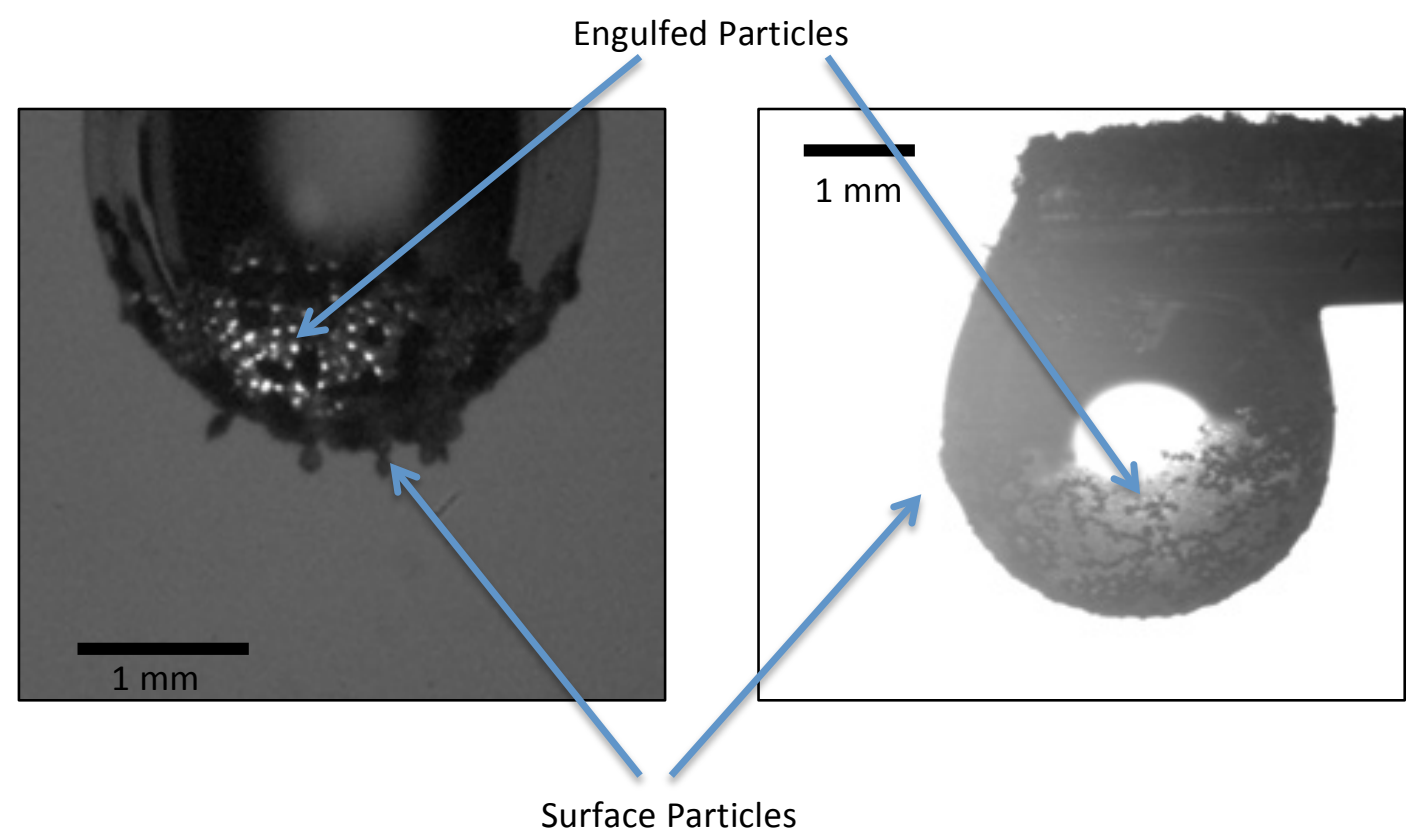

Figure 6.3: Photographs of pendent water drops containing glass ballotini and coal particles (a) after vibrating the mix under the drop, (b) after sprinkling from above.

In some experiments, it was found that as particles loaded onto the drop, a critical point was reached beyond which the drop detached from the needle due to additional weight of the particles. However, there were few exceptions to the detachments. In some cases the drop did not detach from the needle. Quantitative analysis of the attached particles was difficult due to the different particle quantities used, humidity level in the room and different drop detachment times. However, the experiments did demonstrate the potential for selective capture of hydrophilic particles and rejection of hydrophobic particles. The uncertainties in the experimental data, in terms of recovery, were too high, it was necessary to develop a more robust approach based around multiple drops.

\subsection{Results of the Multiple Drop Experimental System}

As noted in Chapter 5, the results of each experiment were analysed to determine the percentage of particles recovered in the underflow using different water fluxes. The recovery of particles in the underflow was calculated as a mass percentage of the total feed passing through the experimental system, as given by Eq.6.1. 
Underflow Recovery $(\%)=\frac{\text { Mass of Underflow }}{\text { Mass of underflow }+ \text { Mass of Overflow }} \times 100$

The superficial velocity of the water was calculated by dividing the volumetric rate by the water flow area as given in Table 5.2. In all experiments, it was assumed that the particle velocity was equal to the air flow velocity throughout the collision chamber. Note that this definition of recovery was based only on the material collected at the end of the experiment, and was not related to the amount of feed that entered the rig. Due to problems arising from material being deposited on the pipe walls, experiments with a mass loss of more than $15 \%$ were omitted from the final data sets.

\subsubsection{Recovery Variation with the Water Flux}

Figure 6.4 shows the comparison of the actual recovery for the "as received" ballotini in $23-47 \mu \mathrm{m}$ range and the predicted recovery versus the water flux.

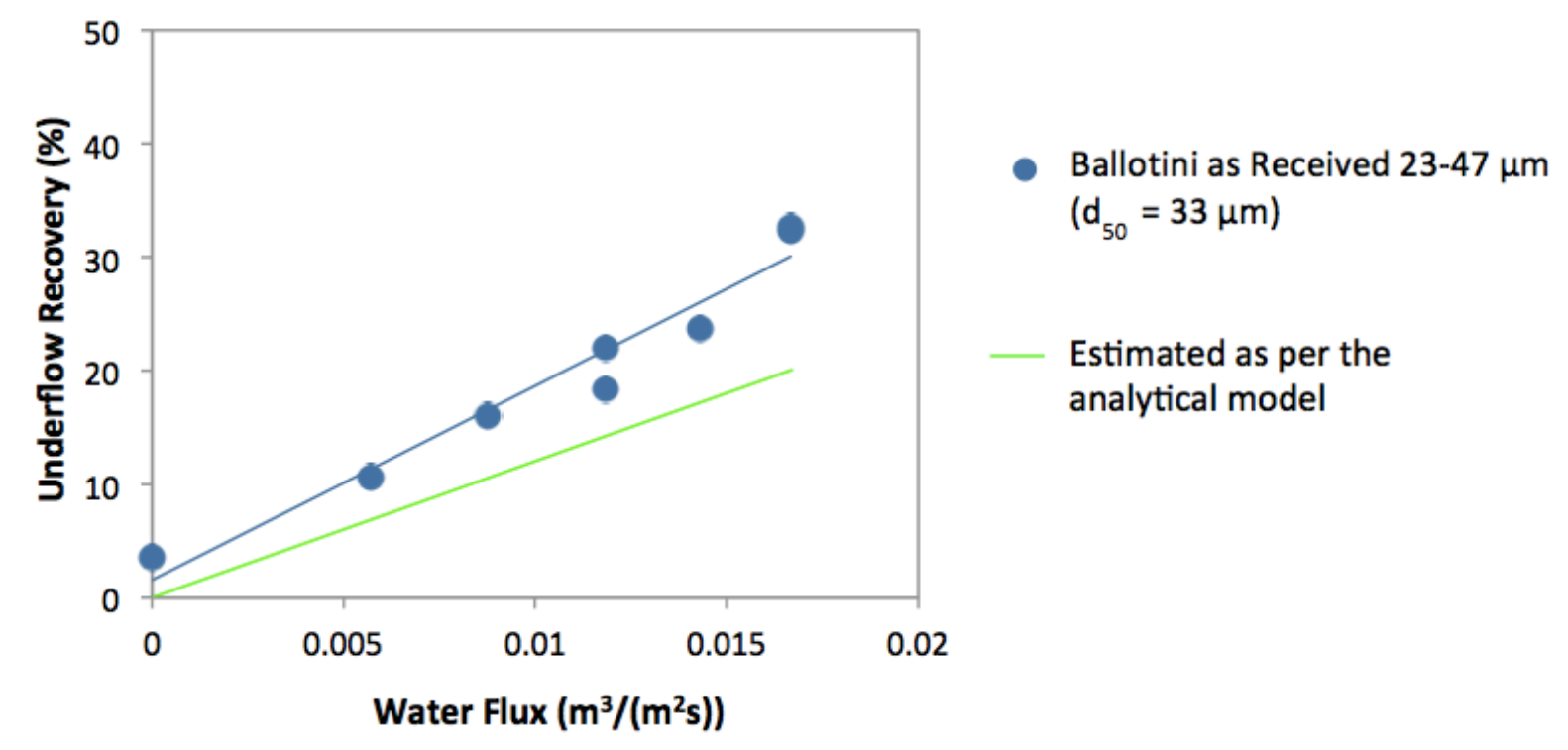

Figure 6.4: Comparison between the predicted recovery and the actual recovery. Blue line corresponds to underflow recovery of as received glass ballotini $(23-47 \mu \mathrm{m})$ versus wash water flux. The straight line represents the best fit for the experimental data. 
It was observed that the recovery of underflow increases with the increasing water flux. The trend of the results was similar to the drop-particle collision model for $u_{d} \gg u_{p}$ (Equation 4.10), which predicts that recovery should be proportional to water flux over this experimental range of conditions.

In all these experiments horizontal particle/air velocity $\left(U_{\mathrm{p}}\right)$, drop velocity $\left(U_{d}\right)$ and drop diameter $\left(D_{d}\right)$ were kept as a constant. Increases in water flow rate (or flux) affected only for the number of drops fell with in a particular time. Further, there was a $2 \%$ underflow particle recovery in the zero water flux experiments also. This is due to a proportion of the very fine particles remaining attached to the walls of the collision chamber and the underflow pipe.

\subsubsection{Comparison of Experimental, Analytical and DEM Results}

As shown in Figure 6.5, the analytical model and the DEM calculations of the underflow recovery were in excellent agreement. However, the experimental underflow recovery values obtained using the ballotini had a slightly higher recovery than predicted using the models. This difference is not considered to be significant given the considerable uncertainty in the experimental conditions defining the gas flow velocity, particle velocities, and the imposed water flux of drops as well as the assumptions of the models. 


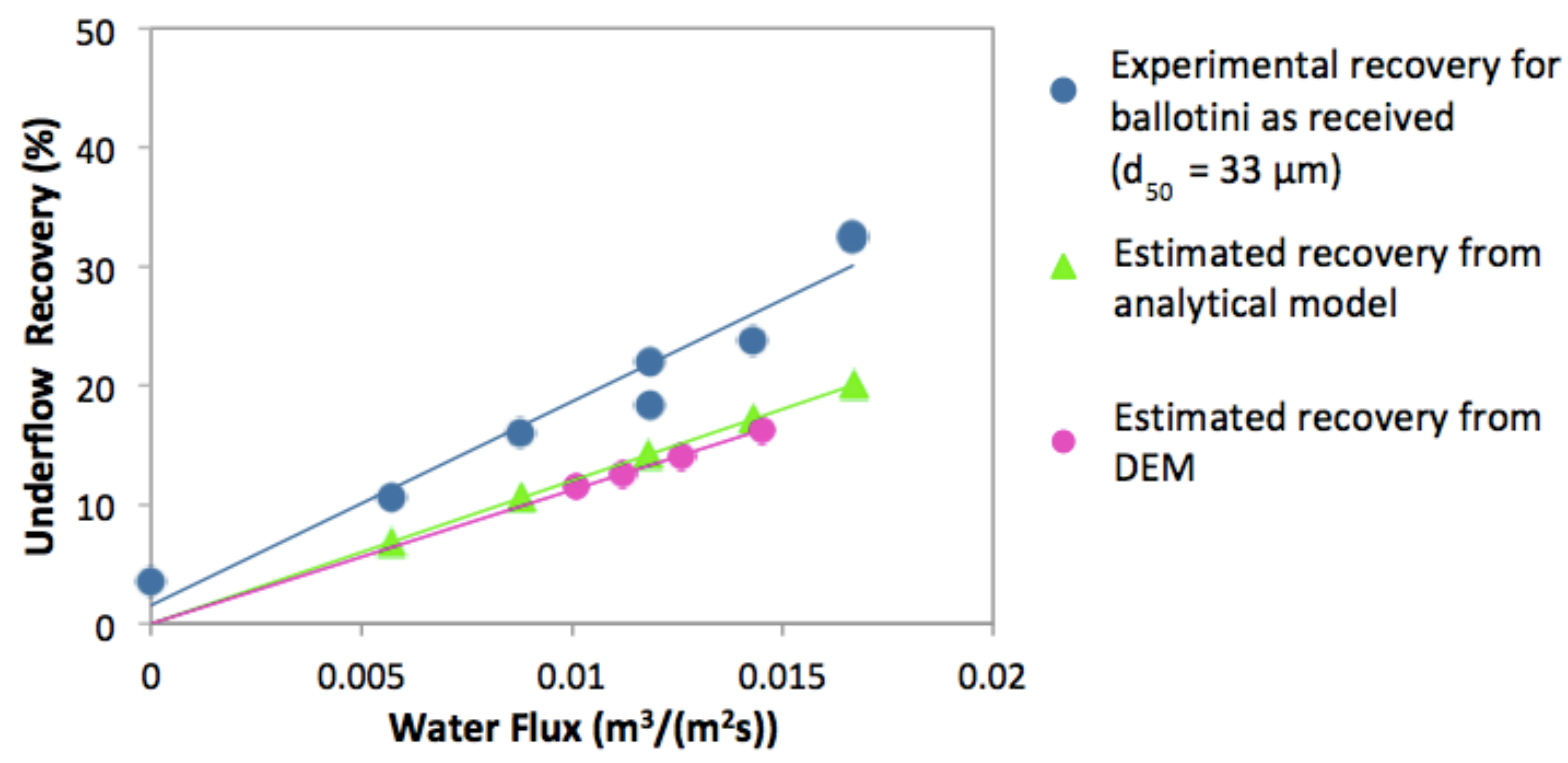

Figure 6.5: The experimental underflow recovery and the estimated recoveries shown as a function of the wash water flux.

\subsubsection{Effect of Surface Modification}

\section{(a) Effect of Surface Cleaning}

A second series of experiments was then conducted using essentially the same ballotini. However, in this case the particles were cleaned (surface modified) using a solution of Extran $^{\circledR}$, as described in Section 5.4.2. Cleaned ballotini should have had a near zero contact angle and hence had superior wetting properties, while the "as received" ballotini would have had a contact angle moderately higher than zero. Hence, cleaned particles should demonstrate a better adhesion to the water drops. It was expected that the cleaned (and hence hydrophilic) ballotini should have a higher recovery than the uncleaned ballotini.

For the size fraction $23-47 \mu \mathrm{m}$, the "as received" glass ballotini and cleaned ballotini underflow recoveries are plotted against different water fluxes in Figure 6.6. As expected the recovery increased linearly with increasing water flux for both cases. However, the recovery is consistently higher for the clean ballotini. These data sets provide clear evidence of limited selectivity between the cleaned and uncleaned ballotini (as received) as a result of surface property changes. Clearly, the cleaned ballotini should have had a near zero contact angle and hence had superior wetting 
properties, while the "as received" ballotini would have had a contact angle moderately higher than zero. Hence, cleaned particles should demonstrate a better adhesion to the water drops. Further, these results demonstrated reproducibility and consistency between different experiments conducted at the same water flux with the same particle type.

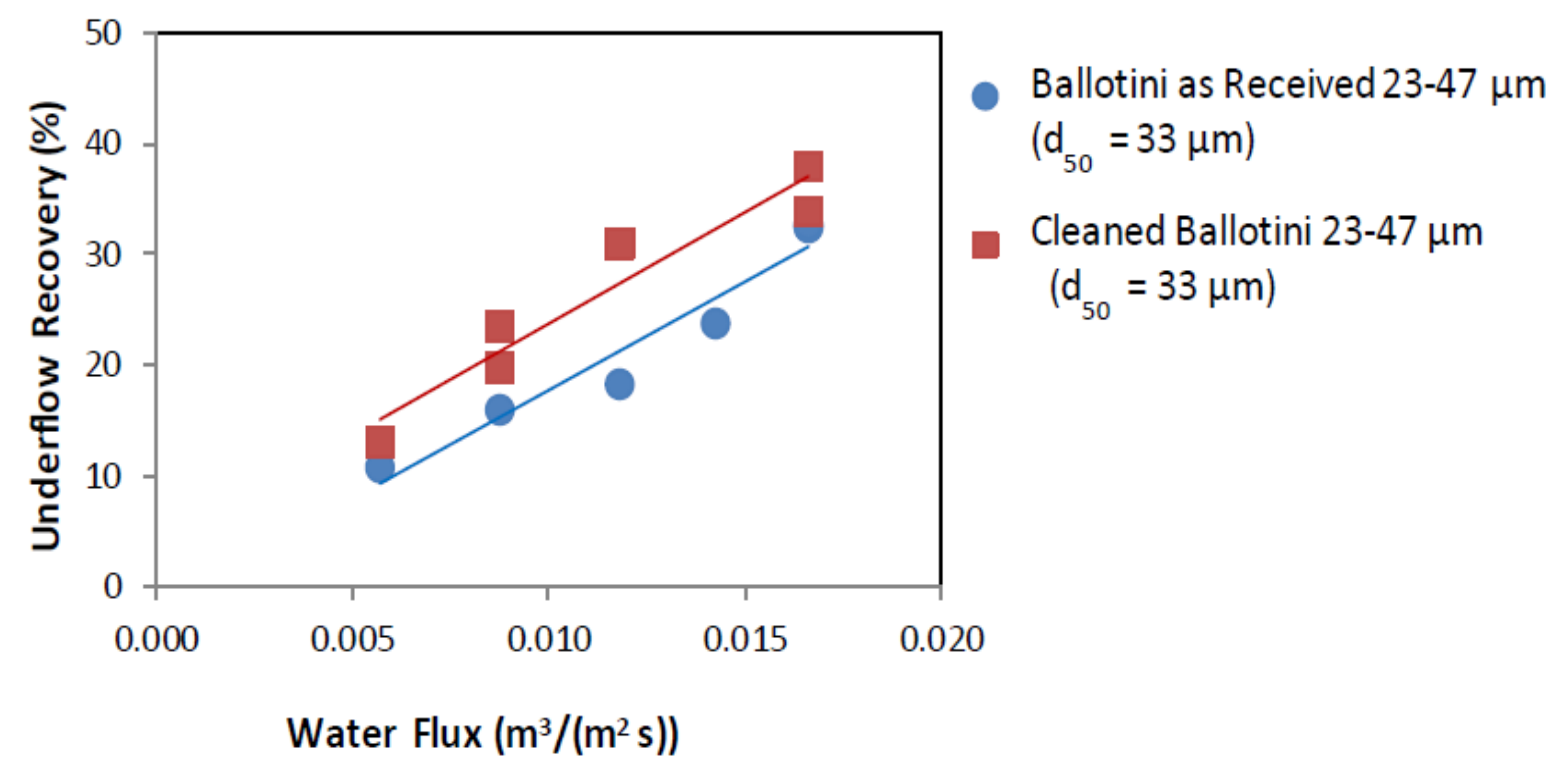

Figure 6.6: Recovery of cleaned glass ballotini and "as received" glass ballotini in the size range $23-47 \mu \mathrm{m}$.

It is also noted that the intermediate water flux produces the most reliable results for this experimental system. At the lowest water flux, water drops tend to dribble while at the highest water flux, the water drops tend to flow as a jet. Experiments have been repeated in order to check the reproducibility of the systems for two particular water fluxes $\left(0.0088\right.$ and $\left.0.0167 \mathrm{~m}^{3} / \mathrm{m}^{2} \mathrm{~s}\right)$. These results were found to be reproducible with in a range of $\pm 3 \%$ (Refer Appendix D for data).

\section{(b) Effect of Surface Esterification (Hydrophobised particles)}

A set of experiments was conducted with esterified (hydrophised) glass ballotini, using the same glass ballotini particles used previously with the size fraction of $23-47 \mu \mathrm{m}$. Though these particles resulted in a lower particle recovery than "as received" 
(uncleaned) ballotini, considerable particle losses occurred in these experiments. These particles were very cohesive in dry state and not all passed through the vibrating sieve, even after an extended time. Hence, these results were obtained in non-standard conditions and are difficult to compare. Therefore, they are excluded from this results chapter and shown in Figure E.2, Appendix E.

\subsubsection{Recovery Variation with Different Particle Sizes}

The underflow recovery was then investigated using two different size fractions; 17 $36 \mu \mathrm{m}$ and $23-47 \mu \mathrm{m}$ cleaned glass ballotini. The results of this series of experiments are plotted in Figure 6.7.

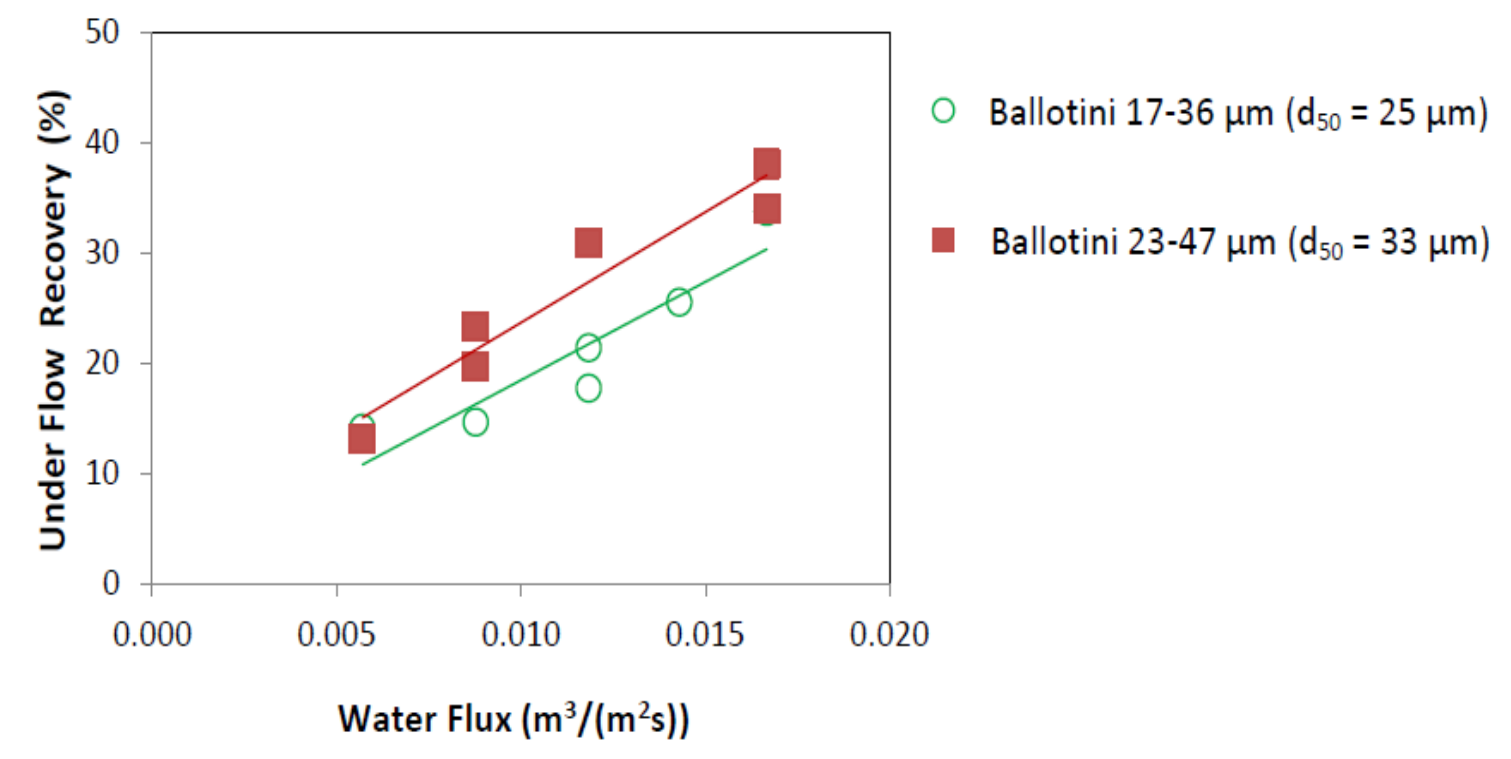

Figure 6.7: Underflow recovery of cleaned glass ballotini in 17-36 $\mu \mathrm{m}$ and $23-47 \mu \mathrm{m}$ size ranges.

Glass ballotini $17-36 \mu \mathrm{m}$, which were smaller of the two particle samples used in the experiments, exhibited a recovery lower than the glass ballotini $23-47 \mu \mathrm{m}$. These results suggest that even a small decrease in the particle size leads to a gradual reduction in the tendency for capture. This can also be related to the higher momentum of the larger particles, which results in a higher probability of collision with water drops. This also reflects a tendency for finer particles to be entrained in the airflow around the 
drops, due to their lower momentum. However, these effects should be relatively small for this limited change in particle size.

\subsubsection{Effect of Particle Size and Shape}

The results obtained for three different size ranges; $8-29 \mu \mathrm{m}, 16-42 \mu \mathrm{m}$, and $21-54$ $\mu \mathrm{m}$, of cleaned rough silica flour are shown in Figure 6.8. For comparison, data for glass ballotini $23-47 \mu \mathrm{m}$ are also included.

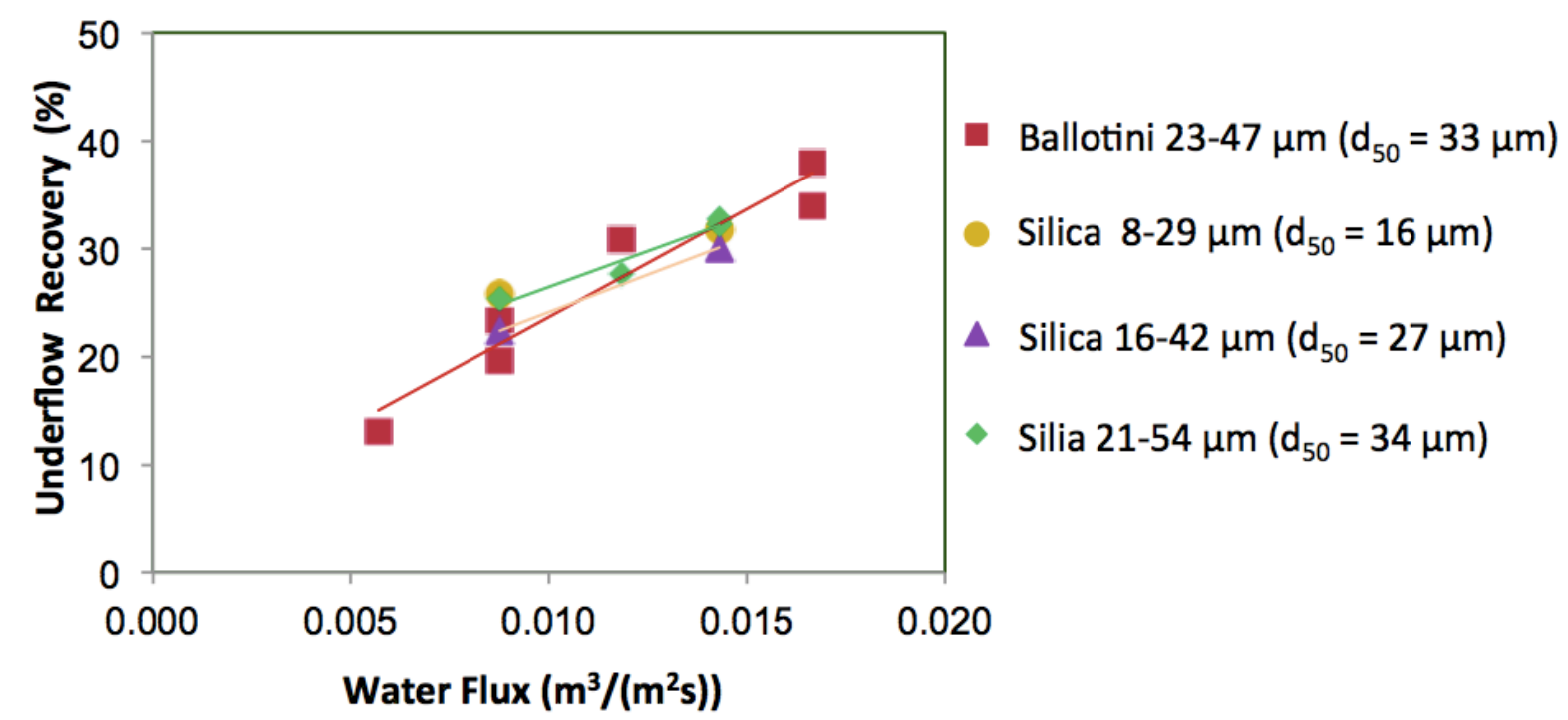

Figure 6.8: Underflow recovery of different size range clean silica; $8-29 \mu \mathrm{m}, 16-42$ $\mu \mathrm{m}$, and $21-54 \mu \mathrm{m}$. For comparison, the recovery of $23-47 \mu \mathrm{m}$ cleaned ballotini is also shown.

The tested silica flour samples $(8-29 \mu \mathrm{m}, 16-42 \mu \mathrm{m}$, and $21-54 \mu \mathrm{m})$ produced very similar recoveries. These results are consistent with the results obtained using spherical glass ballotini particles in the range $23-47 \mu \mathrm{m}$.

Rough silica flour is a material with irregular shapes compared to glass ballotini as shown in Figure 6.9 microscope images. These results suggest that particle shape may also have a role in determining the selectivity of particles. Irregular particles present interaction points such as jagged edges and asperities, which favour collision and 
engulfment. This indicates that there may be less sensitivity to particle size than is observed for precise spherical particles.
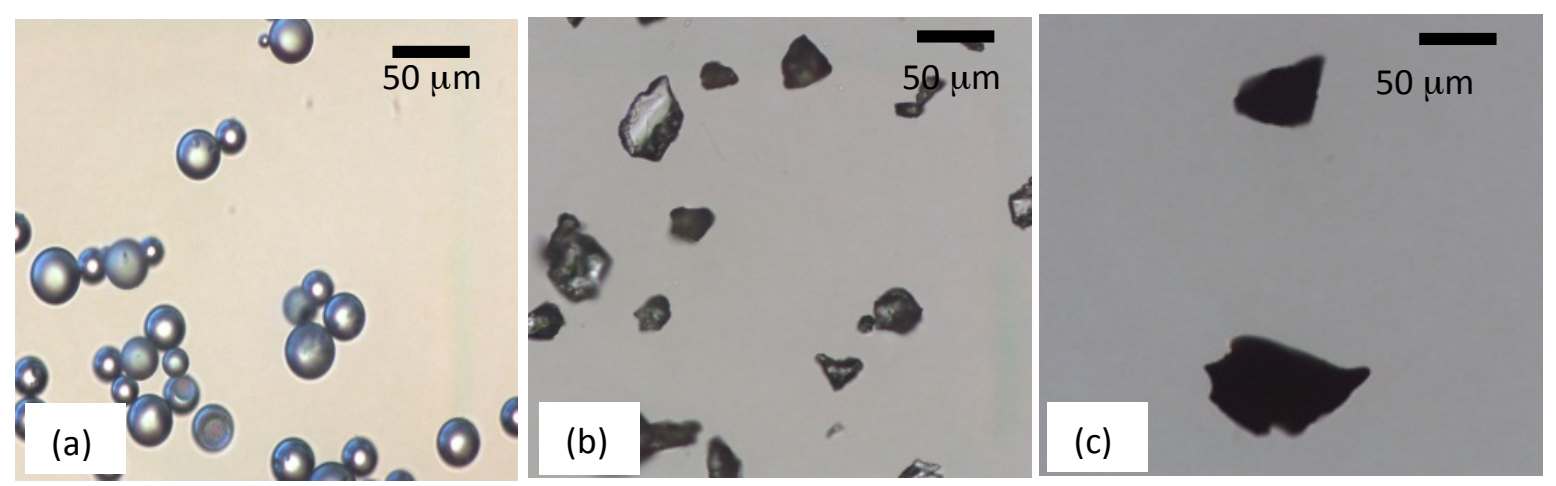

Figure 6.9: Microscope Images of (a) glass ballotini (b) silica and (c) coal in 38-45 microns size range.

\subsubsection{Effect of Different Size Hydrophobic Particles}

When hydrophobic coal particles were introduced to the experimental system, relatively low recoveries were obtained for all size fractions as shown in Figure 6.10. It was difficult to disperse the coal in the size range below $38 \mu \mathrm{m}$. Hence, relatively coarse particles $(30-78 \mu \mathrm{m}, 29-105 \mu \mathrm{m}$ and $24-116 \mu \mathrm{m})$ were used. It was shown that the results were virtually independent of the particle size. 


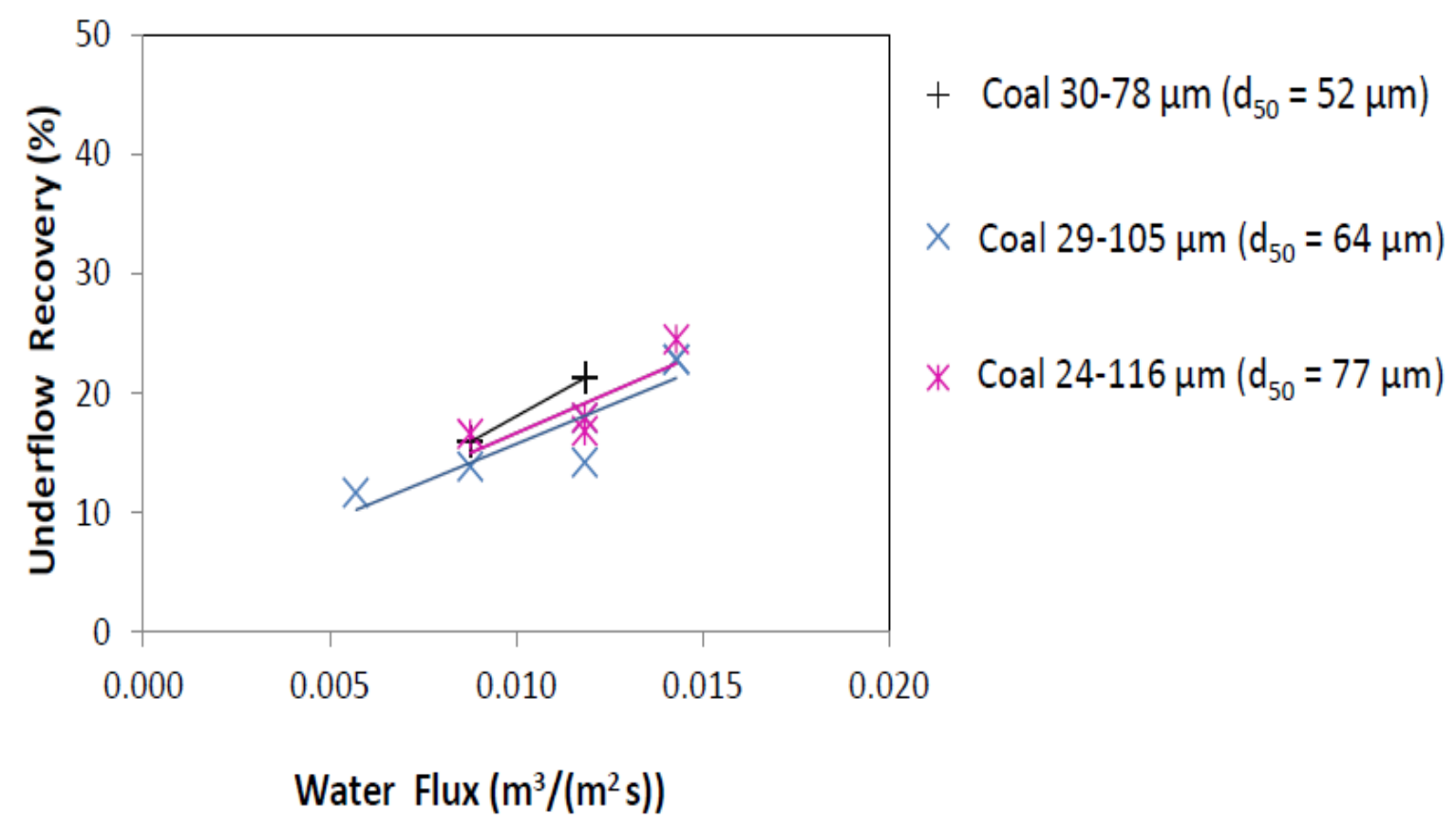

Figure 6.10: Underflow recovery of coal covering different size ranges; 30-78 $\mu \mathrm{m}, 29-$ $105 \mu \mathrm{m}$ and $24-116 \mu \mathrm{m}$.

Moreover, the recoveries were significantly lower than those obtained using ballotini and silica. The ratio of silica recovery to coal recovery denotes the selectivity. Thus, the selectivity at a water flux of $0.01 \mathrm{~m}^{3} /\left(\mathrm{m}^{2} \mathrm{~s}\right)$, based on the ratio of the recoveries, was approximately 1.5 in favour of silica. This level of selectivity, while significant, is unlikely to offer the possibility of a new separation technology, given the extraordinary improvement required for new technologies to displace existing technologies. However, given the clear difference in the tendency for recovery due to differences in hydrophobicity, there exists the potential for further fundamental study to examine in more detail the precise nature of the interaction, and interception between these moving particles.

\subsubsection{Effect of Different Particle Types}

Particles with different shapes, sizes, and surface properties, well beyond those described so far in this Chapter were tested in the experimental system. This additional 
work was part of a broader attempt to explore the particle drop interaction. Unfortunately this work proved to be inconclusive, often due to the difficulty in managing the reproducible, uniform dispersion and transport of these particles. Appendix E shows the recoveries obtained for cleaned silica flour (4-37 $\mu \mathrm{m})$, hydrophobised ballotini $(23-47 \mu \mathrm{m})$, fine graphite $(<38 \mu \mathrm{m})$ and coarse graphite $(38-45$ $\mu \mathrm{m}$ ) with a water flux of $0.01 \mathrm{~m} / \mathrm{s}$. Some attempts were also made to use synthetic graphite powder (Asbury Graphite Mills, USA). However, like the fine coal, there were problems with creating a dispersed plume due to the cohesive nature of the material. There were also unacceptably high experimental losses for the material that did pass through the feed screen. Hence this data is not discussed here.

\subsubsection{Effect of Air Flow Rates}

Experiments were performed using two different airflow rates, $1.4 \mathrm{~m} / \mathrm{s}$ and $1.75 \mathrm{~m} / \mathrm{s}$ with 21- $54 \mu \mathrm{m}$ cleaned silica to investigate the underflow recoveries for particles with different momentum and contact time.

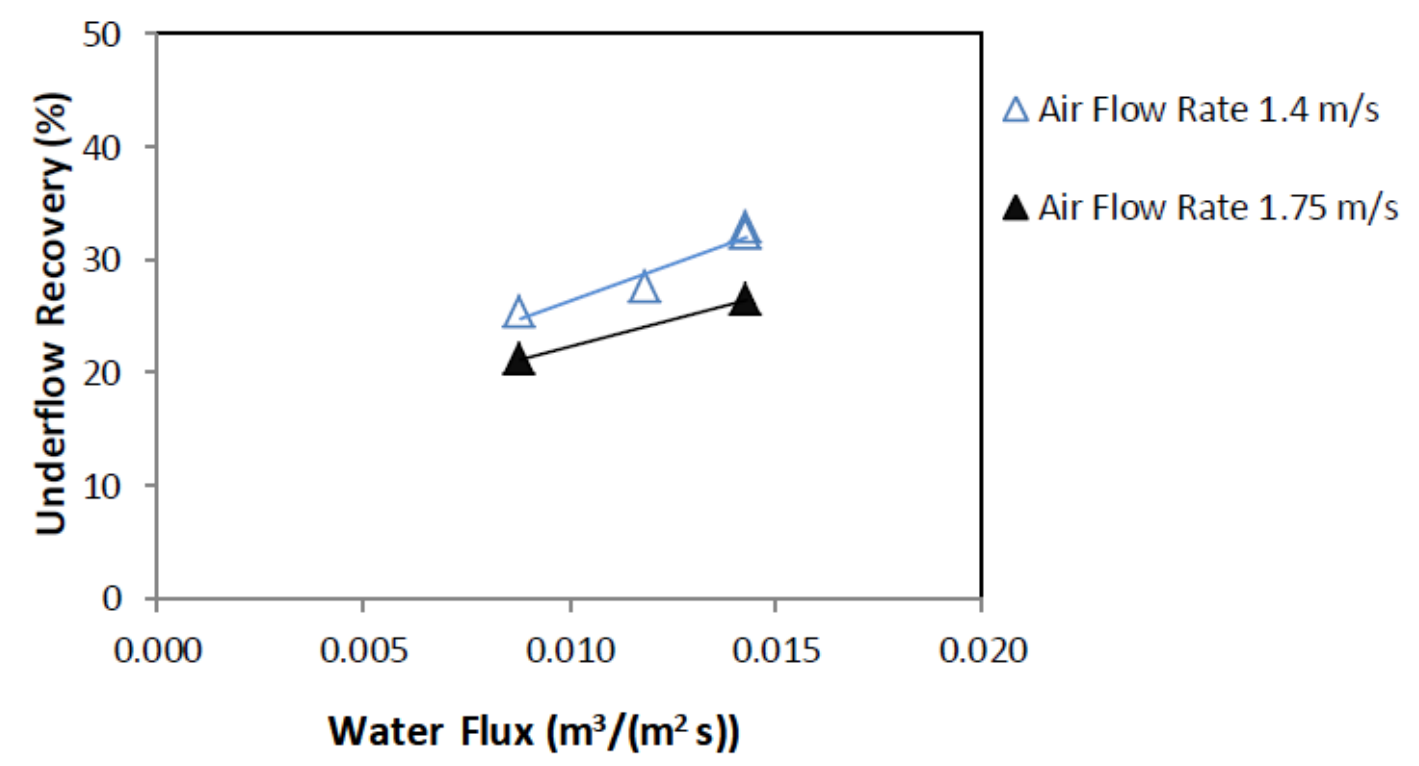

Figure 6.11: Recovery of cleaned glass ballotini at two different airflow rates; $1.4 \mathrm{~m} / \mathrm{s}$ and $1.75 \mathrm{~m} / \mathrm{s}$. The same particle sample (21- $54 \mu \mathrm{m}$ cleaned silica) was used in all experiments. 
As shown in Figure 6.11, particle recovery decreases with increasing airflow velocity. This can be explained by the contact time. Particles passing with a high velocity have less contact time with the falling drops compared to the particles passing at a lower velocity. Therefore with higher air flow rates, less particles are collided with the water drops and collected in the underflow. Both analytical and DEM models showed an increase in the recovery with the reduced air flow rates. Also with the lower airflow rate there may be a lower tendency to entrain particles in the collision chamber compare to higher airflow rate.

\subsubsection{Recovery Variation with the Water Quality Changes}

Experiments were performed to test the effect of water purity on particle recovery. Figure 6.13 shows the underflow recovery for $21-54 \mu \mathrm{m}$ cleaned silica with reverse osmosis (RO) water and tap water. Reverse osmosis water contains less ions compared to tap water. The RO water had a conductivity less than $10 \mu \mathrm{S}$ while normal tap water had a conductivity of $247 \mu \mathrm{S}$ at the same room temperature $\left(27^{\circ} \mathrm{C}\right)$. Hence, it was expected that a higher recovery would be observed with the tap water due to greater surface charges on the impacting particles.

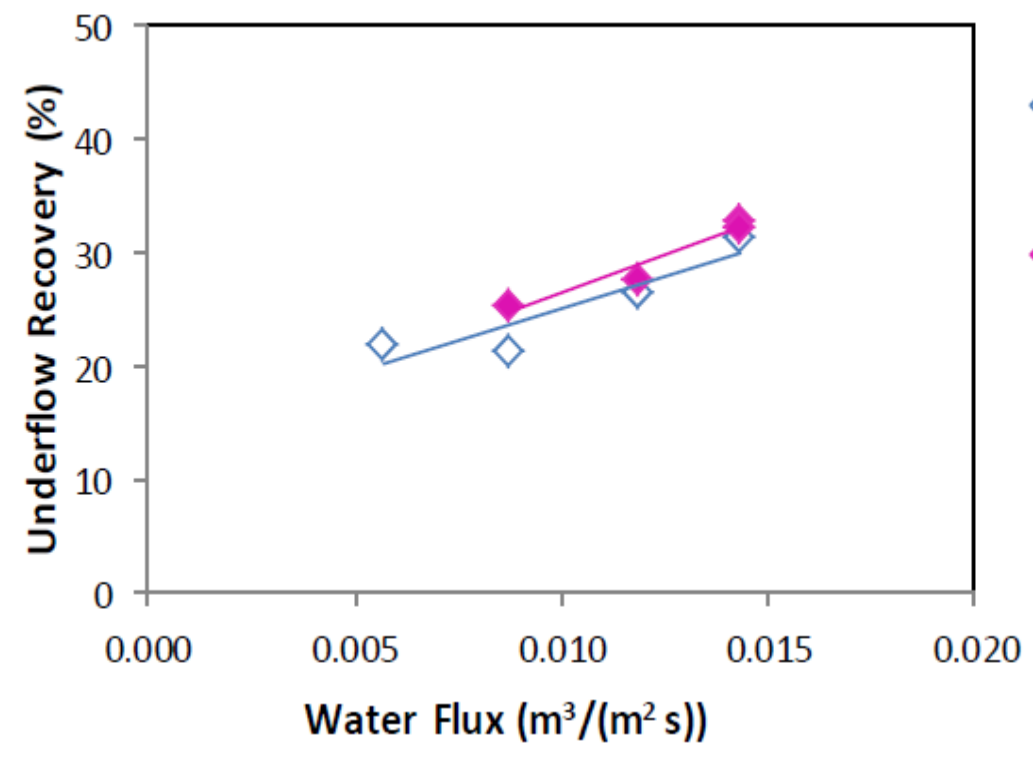

$\diamond$ Silica 21-54 $\mu \mathrm{m}$ with RO water

Silica 21-54 $\mu$ m with Tap water

Figure 6.12: Underflow recoveries for 21- $54 \mu \mathrm{m}$ cleaned silica with reverse osmosis (RO) water and tap water. 
However, RO water gave slightly less recovery compared to normal tap water as shown in the Figure 6.12; however, the difference is within experimental error.

Figure 6.13 indicates recovery of $21-54 \mu \mathrm{m}$ cleaned silica particles with different salt solutions (Aluminium sulphate) concentrations. These salt solutions were used to increase the conductivity of water which was initially $247 \mu \mathrm{S}$. Aluminium sulphate $\left(\mathrm{Al}_{2} \mathrm{SO}_{4}\right)$ was dissolved in tap water to create solutions with concentrations up to 2000 $\mathrm{mg} / \mathrm{L}$ and with conductivities up to $966 \mu \mathrm{S}$. All these experiments were conducted at a constant water flux of $0.0143 \mathrm{~m}^{3} / \mathrm{m}^{2} \mathrm{~s}$. Aluminium sulphate was chosen because of the high valency of the ions, meaning it will have a large effect on surface charges. Hence the recovery would increase with the aluminium sulphate concentration.

Although the conductivity doubled the recovery did not increase linearly as shown in Figure 6.13. The results show a very slight increase in recovery with increasing salt concentration.

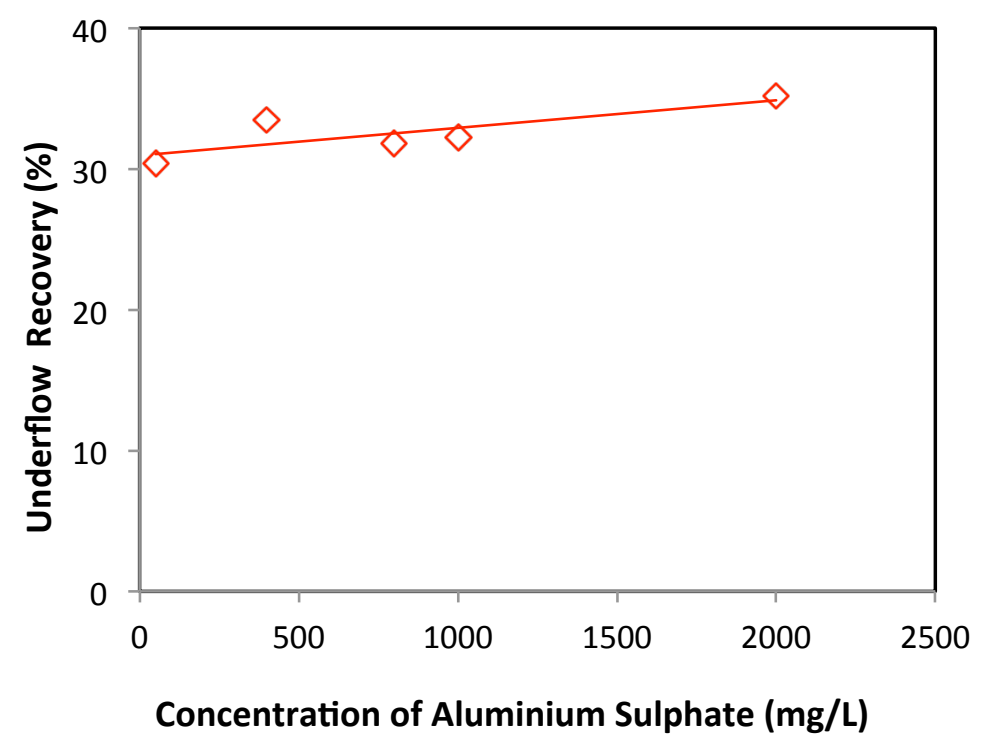

Figure 6.13: Recovery variation of $21-54 \mu \mathrm{m}$ cleaned silica with increasing salt (Aluminium sulphate) concentration. Salt concentration changed from $50 \mathrm{mg} / \mathrm{L}$ up to $2000 \mathrm{mg} / \mathrm{L}$. Conductivity of water changed from $247 \mu \mathrm{S}$ to $966 \mu \mathrm{S}$. All these recoveries were obtained at a constant water flux of $0.0143 \mathrm{~m}^{3} / \mathrm{m}^{2} \mathrm{~s}$. 


\subsubsection{Separation of Coal-Silica Particle Mixtures}

Mixtures of 24-116 $\mu \mathrm{m}$ coal and 21-54 $\mu \mathrm{m}$ silica particles $(50 \% \mathrm{w} / \mathrm{w})$ were introduced to this system and measured the recovery of each component in the underflow as a percentage of the total weight of the each component pass through the system. These two components in the underflow was separated by wet sieving. A higher percentage of hydrophilic silica was found in the underflow as shown in Figure 6.14. However, it was also found that some of the hydrophobic coal particles reported to the underflow as these particles tend to attach to the outer surface of the water drop. Similarly, a small percentage of silica was also found in the overflow.

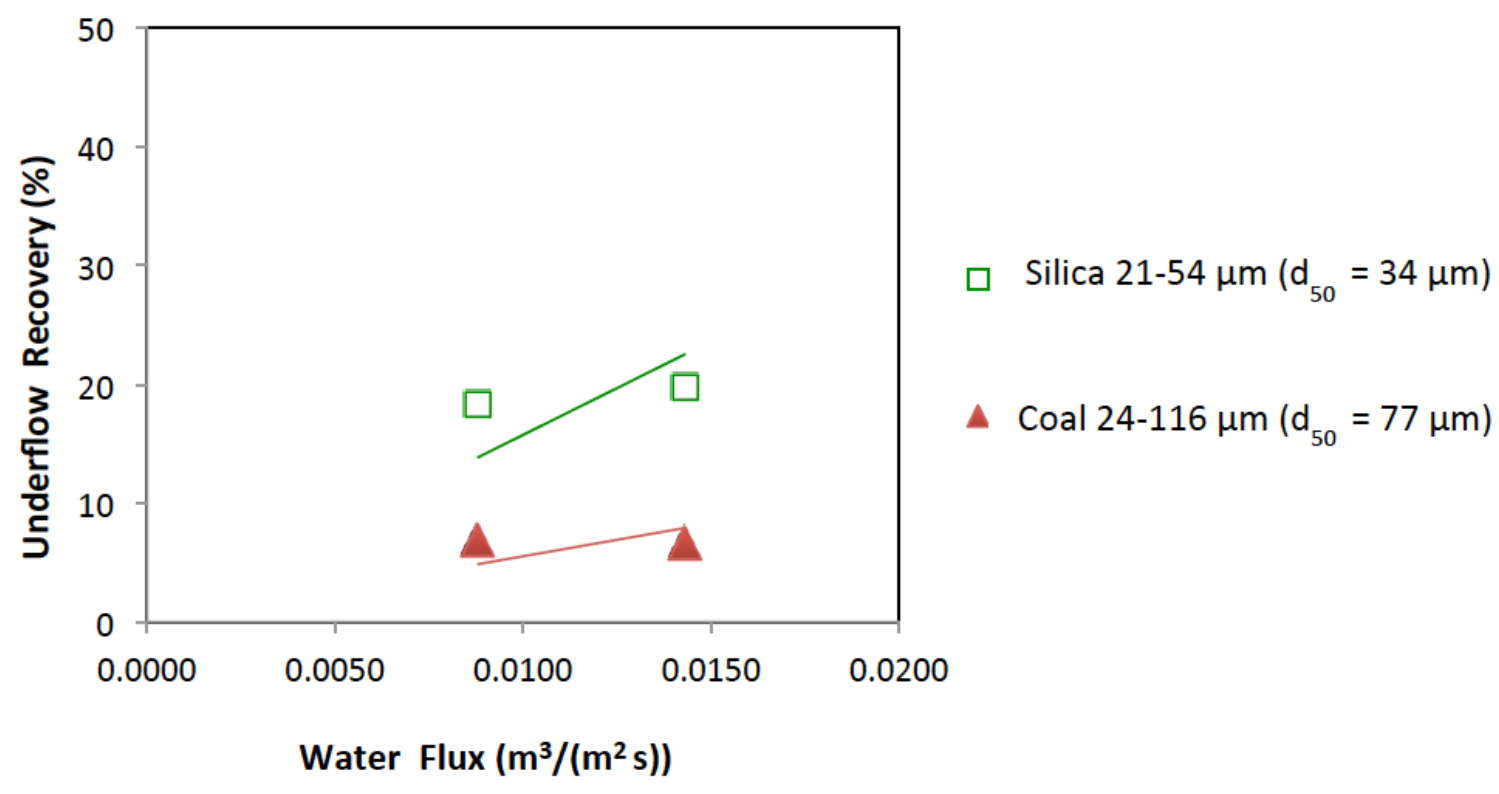

Figure 6.14: Recovery of $24-116 \mu \mathrm{m}$ coal and $21-54 \mu \mathrm{m}$ silica in the underflow for $50 \% \mathrm{w} / \mathrm{w}$ silica-coal feed mixture.

It was observed from the experiments that the selectivity ratio between silica and coal in the underflow was approximately 2.5. This value was higher than the result obtained for the single component experiments. This higher selectivity can be explained in terms of a blocking mechanism arising from the presence of the coarser coal particles at the gas liquid interface of the falling drops. Once an initial level of coal particles attaches at the interface, the opportunity for further coal particles to attach is reduced as shown in 
Figure 6.15. The coal particles will collide with existing coal particles already at the interface. The particles that collide will then fail to adhere and will tend to fall away and back into the air stream. However, the silica particles $(21-54 \mu \mathrm{m})$, which are much finer than the coal particles $(24-116 \mu \mathrm{m})$, are free to pass into the water drop via the relatively large spaces between the adhered coal particles. Thus, the selectivity of silica to coal in the feed mixture is higher than for the selectivity of 1.5 obtained for the single component feeds.

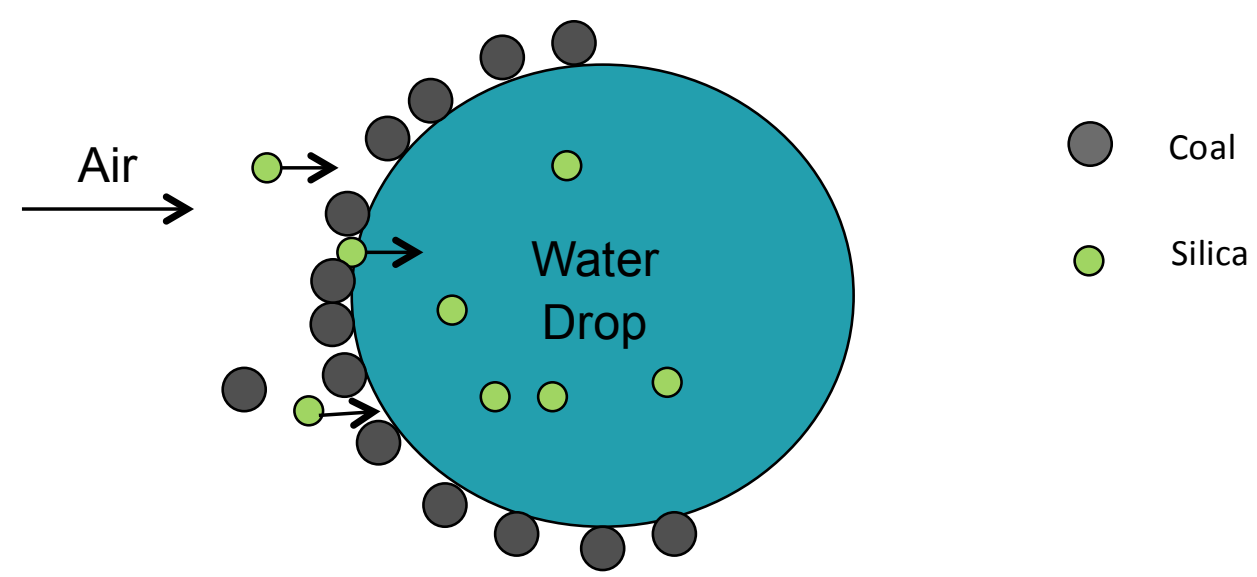

Figure 6.15: Blocking mechanism used to explain the higher selectivity obtained for the mixture of the 24-116 $\mu \mathrm{m}$ coal and 21-54 $\mu \mathrm{m}$ silica.

\subsection{Discussion}

In all above experiments diameter of water drops was a constant of $3 \mathrm{~mm}$. Unless specifically mentioned, horizontal particle/air velocity $\left(U_{\mathrm{p}}\right)$ and drop velocity $\left(U_{d}\right)$ were kept at a constant of $1.4 \mathrm{~m} / \mathrm{s}$ and $1.04 \mathrm{~m} / \mathrm{s}$ respectively. Most experiments were repeated to ensure reproducibility of the results. The experimental system displayed a remarkably high level of reproducibility. Whilst efforts were made to minimise experimental error, it is estimated that the experimental error in the recovery was about $8 \%$. This error is mainly due to the loss of sample mass passing through the chamber. Minor portions of samples remained on the internal surfaces, and some sample mass was lost during collection. There were also slight changes in water flow rates and airflow rates.

For a few experiments, the size (50 g) of the charge in each packet was varied between $30 \mathrm{~g}$ and $100 \mathrm{~g}$, although this made no noticeable difference to performance. Most of 
these experiments concluded within 2 min of the last packet of feed. However, some materials, such as very fine coal, were cohesive and often failed to pass through the sieve, even after an extended time of up to $5 \mathrm{~min}$ of screen vibration. These experiments ceased after 5 minutes and the coal left on the screen was collected and weighed. Material left on the screen is referred to as feed residue.

Experimental losses were expressed as a percentage of the feed that passed through the screen. For each experiment, the total mass of solids recovered in the combined underflow and overflow was compared with the mass of solids in the feed. Here the feed mass was adjusted for the feed residue. Experiments with losses of over $15 \mathrm{wt} \%$ were omitted from the results. Raw data for all the experimental results have been listed in Appendix D.

In four of the experiments, the system was dismantled at the end of each run to identify the location of lost material. These four experiments were conducted without using water for different materials types. The results of these experiments are shown in Appendix F. It was proven that only a very small amount (typically $<2 \%$ ) of the feed was found in the base of the feed column. So most feed was being successfully carried into the collision chamber. Generally 3 to $8 \mathrm{wt} \%$ of the feed was found in the pipe connecting the collision chamber to the cyclone. This material, which should be included as part of the overflow, was not measured in the standard series of experiments and hence represents a source of uncertainty. The remaining material, which was treated as miscellaneous losses, was usually less than $2 \mathrm{wt} \%$ of the feed that passed through the screen. These losses are believed to be ultrafine material that was either blown off the feed sieve or else not captured by the cyclone.

\subsection{Conclusions}

Two experimental methods described in this work demonstrated that there exists a significant, but limited, level of selectivity between hydrophobic and hydrophilic particles interacting with water drops. Preliminary single drop experiments showed the ability of a water drop to selectively capture hydrophilic particles while the multiple drop laboratory scale experimental system provided quantitative measurements of recovery and selectivity. The recovery of particles by the falling water drops was 
measured as a function of the water flux for a number of particle species. The proportion of the feed particles reaching the underflow was determined from the measurements obtained in the experiments.

Spherical ballotini particles were the most sensitive, with a notable increase in recovery when cleaned, and evidence of increased recovery with increasing particle size. Smaller particles exhibited a lower recovery, perhaps due to their lower momentum and tendency to remain with the streamlines of the airflow.

The recovery of irregular shaped silica flour particles was found to be independent of the particle size. Similar results were observed for irregular coal particles, though the recoveries were lower than for the hydrophilic ballotini or silica flour. Recovery of the hydrophilic silica particles was significantly higher than that of the recovery of hydrophobic coal particles, with a selectivity ratio of approximately 1.5. Moreover, selectivity ratio of approximately 2.5 was obtained for silica and coal mixtures.

This was a preliminary study that was mainly concerned with investigating the effects of the particle properties on the system at a macroscopic level (i.e. the recovery achieved). It would be useful in the future to study this concept at microscopic level. Further it will be interesting to study the effect of increased electrostatic charges in the collision chamber. 


\section{Chapter 7}

\section{ELECTROSTATIC EXPERIMENTS}

\subsection{Introduction}

\subsection{Experimental Set up and the Materials}

\subsection{Experimental Methodology}

\subsection{Experimental Results and Discussion}

7.4.1 Identifying the Experimental Limits and the Phenomena

\subsubsection{Hypothesis}

7.4.2.1 Positive Feedback Mechanisms

7.4.2.2 Negative Feedback Mechanisms

7.4.3 Charge Measurements and Volume Changes with Time

7.4.4 Charge Measurements and Variable Speeds

7.4.5 Hydrophilic Particle Behaviour with Different Liquid Drops

7.4.5.1 Glass Ballotini with Glycerine

7.4.5.2 Glass Ballotini with Hexane

7.4.6 Hydrophobic Particle Behaviour with Water Drops

7.4.7 Hydrophilic and Hydrophobic Particle Mixtures with Water Drops

\subsection{Conclusions}




\section{Journal Article Relevant to the Chapter and Author Contributions}

Paper: Electrostatic Formation of Liquid Marbles

\section{Contribution:}

\begin{tabular}{|l|l|}
\hline Author & Primary Contribution \\
\hline Liyanaarachchi, K.R & $\begin{array}{l}\text { Lead Author, experimentalist, developer of the } \\
\text { experimental methods ensuring well defined } \\
\text { experimental conditions and analysis of experimental } \\
\text { work and surface tensiometer data. }\end{array}$ \\
\hline Ireland, P. & $\begin{array}{l}\text { Contributed to the writing of the paper, experimental } \\
\text { support and analysis of charge data. }\end{array}$ \\
\hline Webber, G. & $\begin{array}{l}\text { Co-supervisor, contributed to the data analysis and } \\
\text { writing of the paper. }\end{array}$ \\
\hline Galvin, K.P & $\begin{array}{l}\text { Principal supervisor, co-developer of the experimental } \\
\text { design and contributed to the writing of the paper. }\end{array}$ \\
\hline
\end{tabular}

Kevin Galvin

Kathika Liyanaarachchi 


\subsection{Introduction}

This chapter investigates an alternative mechanism for transporting and collecting hydrophilic glass ballotini particles into water drops. Electrostatic forces were used to drive the particles, against the force of gravity, in an upwards direction towards a pendent drop. This chapter describes the experimental setup, procedure, method of measurement and analysis, detailed results, discussion, as well as conclusions.

A number of experiments were conducted using glass ballotini on a Teflon substrate. Observations of the behaviour were made as the substrate was raised towards a hanging water drop. In the first set of experiments, the particle transport was observed using high speed video. The charge gained by the drop was measured, and the volume change of the drop used to calculate the number of ballotini particles transported to the drop. The effects of the substrate position and speed were also tested. Once the ballotini system had been studied extensively, different particle species and different liquids were used in order to explore further the generality of the findings.

\subsection{Experimental Set up and Materials}

The experimental method described in this Chapter evolved from a program of work aimed at establishing the conditions needed to insure reproducibility. The ideas for this work were serendipitous, arising from earlier single drop experiments that revealed a remarkable and unexpected phenomenon. Glass ballotini particles were seen to suddenly jump upwards towards a hanging drop of water when brought to within about $5 \mathrm{~mm}$ of the drop. This phenomenon was initially difficult to explain, and difficult to reproduce. For example, it was unclear whether humidity played a role in these events. Eventually, a robust experimental method was developed, allowing meaningful data to be generated.

The experimental system consisted of a hanging water drop connected to a metal syringe with an internal diameter of $1.3 \mathrm{~mm}$. This arrangement was located above a bed of spherical ballotini particles to be tested as shown in Figure 7.1.Spherical glass ballotini in the size range of $75-90 \mu \mathrm{m}$ were used in this test. A surface tensiometer 
video camera (OCA 20, Data Physics, Germany) was used to analyse the water drop, the overall arrangement, and the phenomenon. A high speed video camera (Phantom Five) was also used in some experiments to obtain more detailed observations of the high speed movement of the particles.

In order to establish the particle charge, Teflon tape (Shamban Teflon Ribbon by Unasco, $12 \mathrm{~mm}$ width) was wrapped around a Livingstone glass microscope slide (76.2 $\times 25.4 \mathrm{~mm}$, thickness of $1.0 \mathrm{~mm}$ ) thrice and then rubbed by the index finger 120 times, while wearing a Nitrile glove ('Truecare Aloe', Ongard, Hornsby, Aust.) to tribocharge the surface. The glass ballotini were then placed onto the Teflon covered glass slide with a spatula, insuring there was no contact between the glass and the particles. Grade AI (GB11)) spherical glass ballotini particles provided by Potters were used in these experiments. The slide was then mounted on a Thorlabs L490MZ motorized lab-jack controlled by a computer.

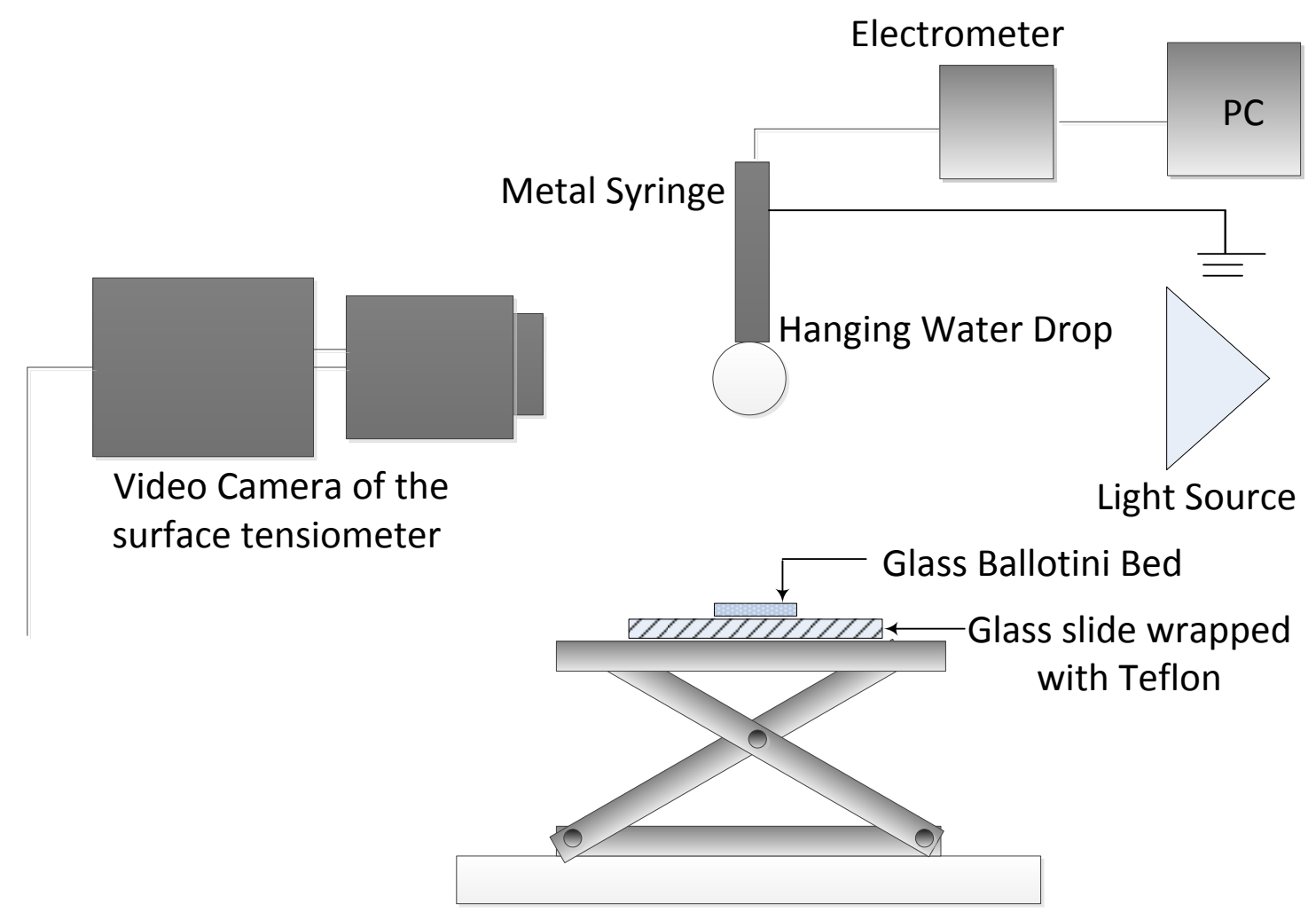

Figure 7.1: Experimental set up. 
A pendent drop of water was formed above the bed of particles via a stainless steel syringe, with the syringe connected by an insulated wire to earth. This insulated wire was passed through the integrating circuit of a Keithley Model electrometer and the signal was logged by a computer. The needle top was isolated from the metallic support of the tensiometer by wrapping Teflon around it.

The properties of different particle species are defined in Table 7.1. Glass ballotini provided by Potters with a density of $2.6 \mathrm{~g} / \mathrm{cm}^{3}$ was classified to produce the size fraction 75-90 $\mu \mathrm{m}$. Queensland coking coal with $6 \%$ ash content was wet screened to make two different samples. Rough silica flour particles (Potters, Grade 400G) were classified by dry screening. PVC particles were also prepared. Both glass ballotini and rough silica flour were treated separately using 10\% w/w Extran ${ }^{\circledR}$ MA 03(phosphate).

Table 7.1: Properties of different particles used in electrostatic experiments and their sources.

\begin{tabular}{|l|l|l|l|l|}
\hline Particle Type & $\begin{array}{l}\text { Size Range } \\
(\mu \mathrm{m})\end{array}$ & $\begin{array}{l}\text { Density } \\
\left(\mathrm{g} / \mathrm{cm}^{3}\right)\end{array}$ & $\begin{array}{l}\text { Dielectric } \\
\text { Constant at 22 } \\
{ }^{\circ} \mathrm{C}, 106 \mathrm{~Hz}\end{array}$ & Source \\
\hline Glass Ballotini & $75-90$ & 2.6 & 6.9 & Potters \\
\hline Coal & $75-90 \& 38-45$ & 1.3 & 3.0 & $\begin{array}{l}\text { Moorvale Mine, } \\
\text { Queensland }\end{array}$ \\
\hline Rough Silica & $75-90 \& 38-45$ & 2.6 & 2.0 & Potters \\
\hline PVC particles & $106-125$ & 0.6 & 1.3 & $\begin{array}{l}\text { Partition } \\
\text { Enterprises, } \\
\text { Queensland }\end{array}$ \\
\hline
\end{tabular}

The liquids used in these experiments are listed in the Table 7.2 along with their properties. The conductivity measurements were obtained using a PCS tester 35 and other parameters are obtained from the respective supplier's information sheets. 
Table 7.2: Physical properties of different liquids used in the electrostatic experiments and their sources.

\begin{tabular}{|l|l|l|l|l|}
\hline Liquid Type & $\begin{array}{l}\text { Specific } \\
\text { Gravity } \\
\left(\mathrm{g} / \mathrm{cm}^{3}\right)\end{array}$ & $\begin{array}{l}\text { Conductivity } \\
(\mu \mathrm{S} \text { at Room } \\
\text { Temperature })\end{array}$ & $\begin{array}{l}\text { Surface } \\
\text { Tension } \\
(\mathrm{mN} / \mathrm{m})\end{array}$ & Source \\
\hline Normal Tap Water & 1 & 240 & 72.8 & N/A \\
\hline Milli Q Water & 1 & 9 & 72.8 & $\begin{array}{l}\text { Millipore Milli Q } \\
\text { water system }\end{array}$ \\
\hline Glycerine $(>98 \%)$ & 1.256 & 0.5 & 63.4 & $\begin{array}{l}\text { Bio-fuels } \\
\text { Technology, llc. }\end{array}$ \\
\hline Hexane $(>95 \%)$ & 0.659 & 0.000004 & 18.4 & J.T. Baker \\
\hline
\end{tabular}

\subsection{Experimental Methodology}

Once the microscope slide was completely covered with Teflon, the experimentalist used their index finger to rub this substrate. A bed of the glass ballotini spheres (75-90 $\mu \mathrm{m})$ was then tipped onto the Teflon surface using a plastic spatula, insuring no spillage. The substrate was then placed onto a metallic bench, located below the hanging drop, making sure none of the particles contacted the bench. The water drop was formed using a micrometer and allowed to hang from the flat tip of a stainless steel cylindrical syringe with a diameter of $1.3 \mathrm{~mm}$. Normal tap water was used in these experiments. The $\mathrm{pH}$ of this water was 6.94 and the conductivity $220-240 \mu \mathrm{S}$. The charged substrate (glass slide wrapped with Teflon) with micron size ballotini was then gradually raised towards the hanging pendant drop and the observations were recorded via the surface tensiometer camera. Any charge transferred to the drop by the ballotini spheres was measured by the electrometer.

In the preliminary experiments reproducibility and the boundary conditions were identified. The drop profile was observed throughout the experiments using the OCA 20 tensiometer. Next, drop volume and charges were monitored with respect to lab jack rise to identify the relationship between volume gain and charge transfer rates. High speed video images were also recorded to analyse this process. Charge data for different 
lab jack speeds ranging from 25 to $200 \mu \mathrm{m} / \mathrm{s}$ were obtained and studied. The distance travelled by the lab jack was also measured and analysed against the charge transferred.

The ballotini particle transfers with different liquids were also studied. Hydrophilic glycerine and hydrophobic hexane were used to produce hanging drops. Instead of hydrophilic glass ballotini, hydrophobic coal (75-90 $\mu \mathrm{m})$ and PVC particles (106-125 $\mu \mathrm{m})$ were also tested using water drops. Finally a mixture of coal and silica (75-90 $\mu \mathrm{m})$ was tested using water drops.

\section{Material Preparation}

The spherical glass ballotini was washed in a 5\% Extran solution, rinsed with copious quantities of double distilled Milli Q dispensed water and then dried in an oven at 100 ${ }^{0} \mathrm{C}$ before being equilibrated to laboratory room temperature in a desiccator.

\section{Charge Measurements}

It should be also noted that in this configuration, the electrometer only registered a net flow of charge to or from the system, not a rearrangement of charge within the system. This was confirmed by control experiments in which the drop and charged substrate were brought together in the absence of particles. No signal was detected in these cases.

All the experiments were carried out at the room temperature $\left(20 \pm 5^{\circ} \mathrm{C}\right)$ and humidity level in the range of $40 \%-50 \%$. The experimentalist wore covered shoes and refrained from touching water during rubbing.

\subsection{Experimental Results and Discussion}

\subsubsection{Identifying the Experimental Limits and the Phenomena}

When the water drop was first formed approximately $40 \mathrm{~mm}$ above the bed of charged particles as shown in Figure 7.2, a few spherical particles of ballotini were seen to spontaneously lift from the substrate and head towards the hanging drop of water. 


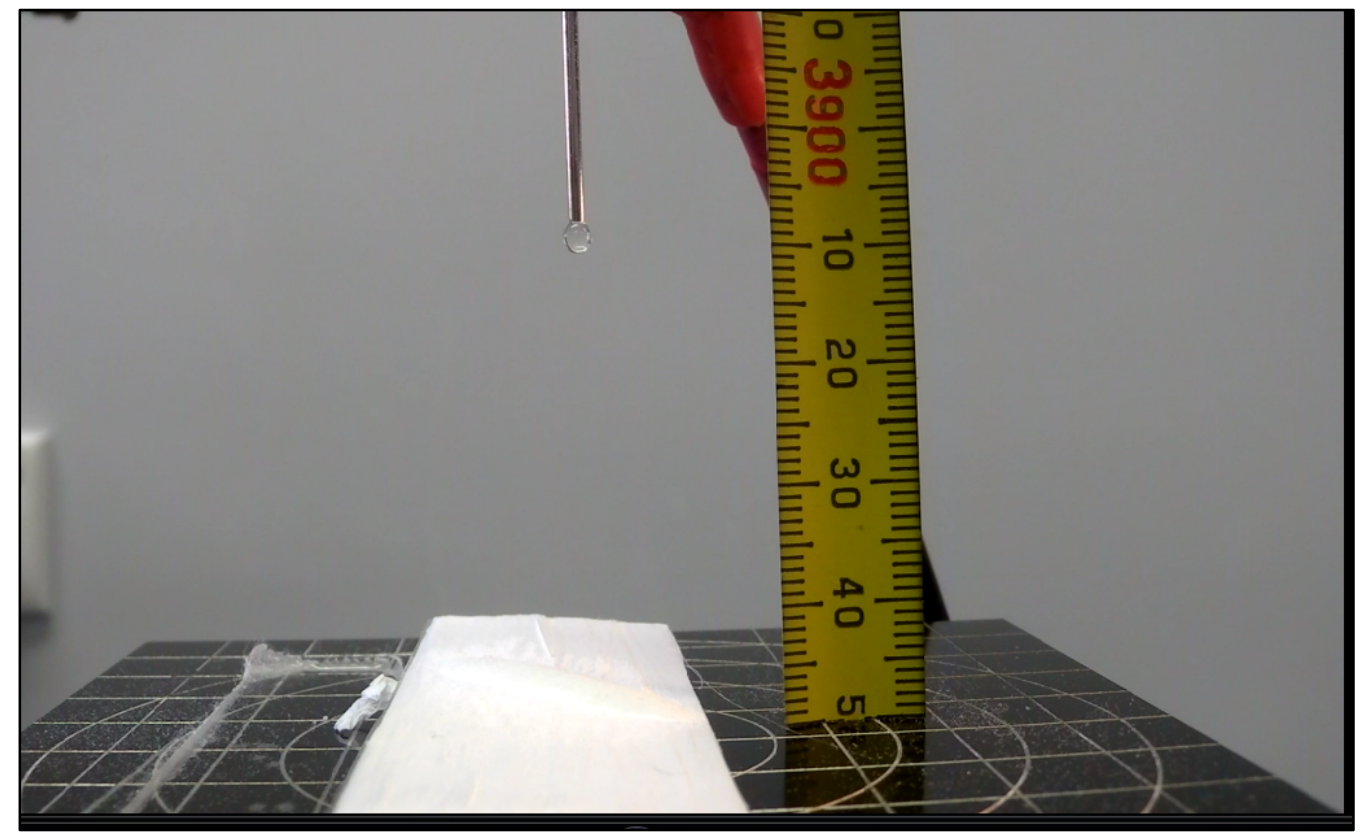

Figure 7.2: Image showing the initial distance of $40 \mathrm{~mm}$ between the drop and the Teflon wrapped substrate.

The spherical particles penetrated through the air-water interface and were engulfed by the drop. With further rise of the substrate more particles were observed to rise and penetrate the drop. Investigations of the observed phenomenon have centred firstly on achieving reproducibility and in turn a clearer understanding of the underlying cause. The spontaneous lift occurred at first with apparent irregularity. Many experiments failed or showed little upwards transport of the particles. It took some effort to finally achieve $100 \%$ reproducibility in producing the phenomenon. The glass slide should be wrapped with Teflon tape, ensuring the entire surface was covered. The slightest spillage of the particles from the Teflon and onto the glass slide led to a loss of particle charge, and invariably to complete failure of the experiment.

Then the experimentalist proceeded to stroke the entire surface of the Teflon tape in a reciprocating fashion at about 2 cycles per second for a period of 60 seconds instead of random rubbing. This process produced a static charge. Any particles spillage onto the bench appeared to release the charge formed from rubbing the Teflon. Hence, spillage was avoided. After establishing these basic procedures, a higher level of reproducibility was obtained. 
When the distance between the drop and the particle bed fell below a certain value, typically 2-5 $\mathrm{mm}$, the particles appeared to transport towards the drop in a steady fashion due to the electrostatic forces. When the separation was reduced further an explosive release of particles from the substrate was observed. Figure 7.3 provides an insight into the phenomenon. The image, which shows the tracer lines due to particle movement, is almost reminiscent of the field lines of force, intersecting the surface of the drop.

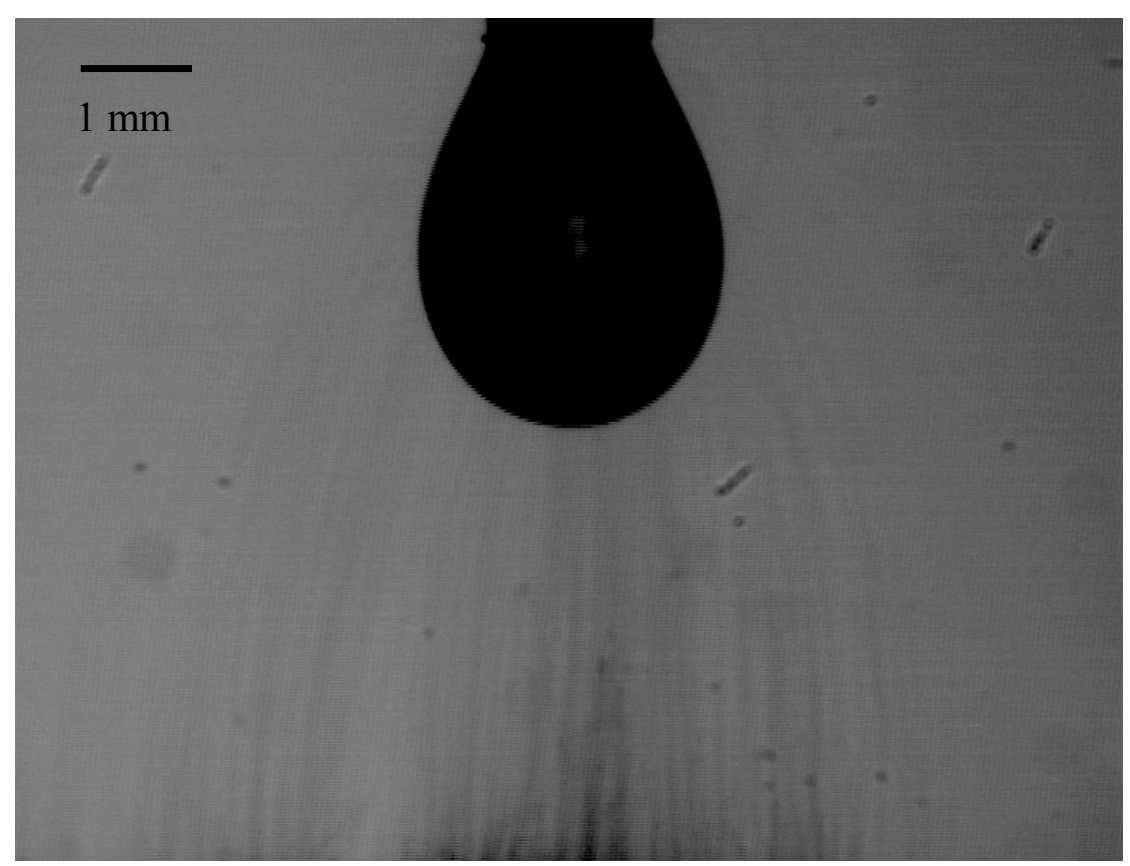

Figure 7.3: Image showing particles of spherical ballotini being transfered to a hanging water drop across an air gap. (Image captured with the OCA 20 tensiometer camera)

Within a period of $300 \mathrm{~ms}$ the particles were swept upwards from the substrate and into the drop. The particles, which were fully engulfed by passing through the air-water interface, settled through the water towards the base of the drop. The additional weight force continued to be supported by the surface tension force between the drop and the syringe tip. As a result, the drop 'sagged' toward the substrate, decreasing the gap between the drop and the particles that remained on the substrate, further increasing the electric field strength and in turn inducing yet more particle transfer. Past a certain 'tipping point', this process overcame the restoring force associated with the surface tension, resulting in the detachment of the drop from the syringe. 
A self-organised critical state evolved as the substrate was lifted towards the drop. The upwards electrical force balances the downwards gravitational force. With the evidence of the very first few particles jumping from the substrate it was noted that keeping the substrate stationary, the process appeared to subside. The process would then resume again by raising the substrate. Then a critical "cascade" or "avalanche" developed, with an explosive lift and transport of the particles into the drop. The drop would literally fill with the spheres, showing a significant increase in its volume with time. In the zone between the substrate and the drop, where the path length was narrowest, the field strength appeared densest. What was remarkable is the path length between the base of the drop and the substrate exceeded $5000 \mu \mathrm{m}$.

Figure 7.4 shows the full sequence of images at the point of explosive transfer of the ballotini to the drop produced by high speed video Phantom Five. Initially the process was relatively slow, but then there was explosive growth as the particles jumped and filled the drop. The drop then hung with increased mass before detaching from the syringe, collapsed onto the bed, leaving a mound of ballotini evident at the substrate. The images were captured with a rate of 25 frames per second.

As seen in Figure 7.4, an agglomerate of this type was left on top of the particle bed at the end of each experiment. The phenomenon observed here may constitute an alternative way of forming liquid marbles discussed in Section 3.4.4, with several attendant advantages. For example, marbles could be made with hydrophilic particles by this method (although the drop is filled, rather than merely coated, with particles). Conversely, when a drop of liquid is placed on the surface of a hydrophilic particle bed, the liquid merely soaks in. While these agglomerates exhibited slow drainage of the binding liquid, and hence slumping, they could easily be stabilised in various ways. For example, there is also the prospect of producing layered marbles, with a hydrophilic core and a stabilising hydrophobic outer shell. Alternatively the particles might undergo a form of sintering or freeze-drying. Since the formation of the marble does not require physical contact between the drop and the bed, it may also be possible to produce agglomerates that are far more symmetric than those formed by the more conventional method. 


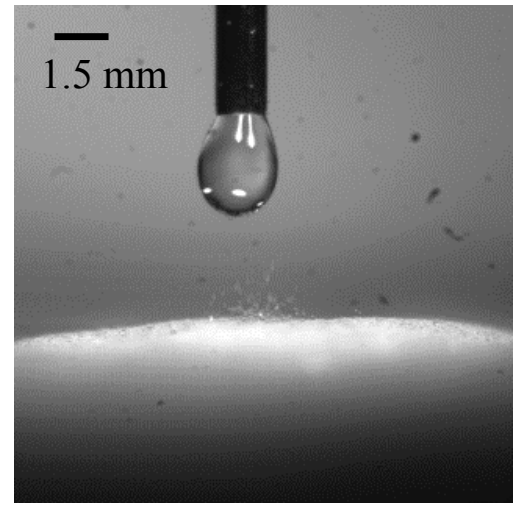

$25 \mathrm{~ms}$

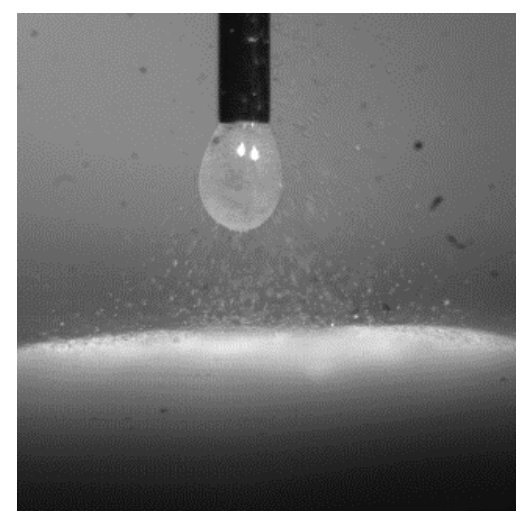

$125 \mathrm{~ms}$

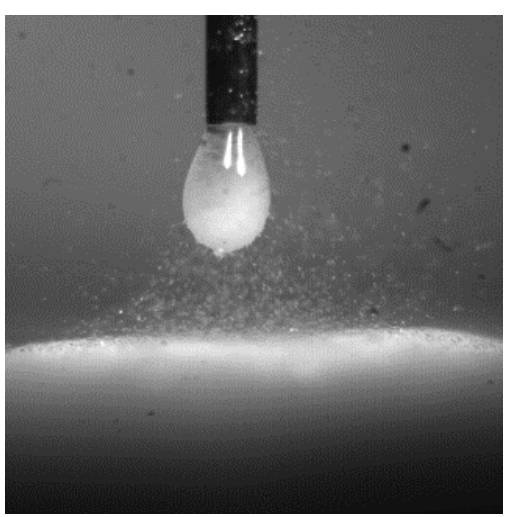

$225 \mathrm{~ms}$

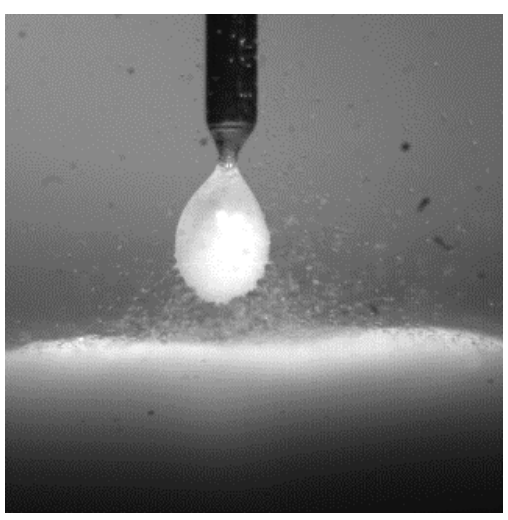

$325 \mathrm{~ms}$

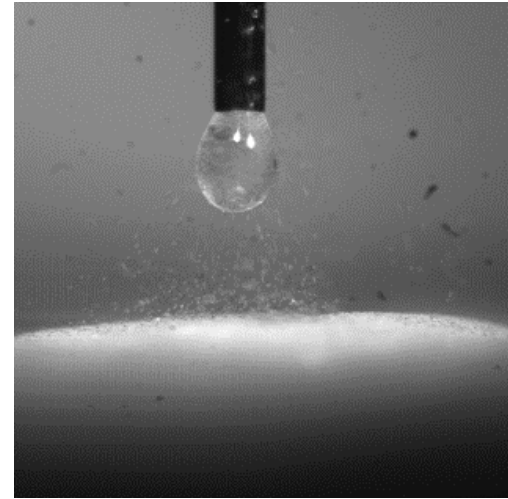

$75 \mathrm{~ms}$

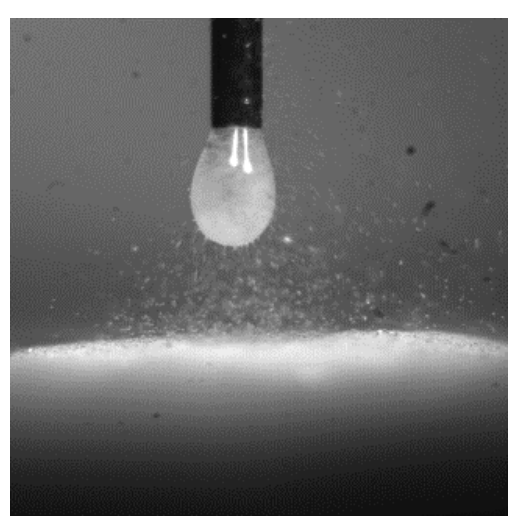

$175 \mathrm{~ms}$

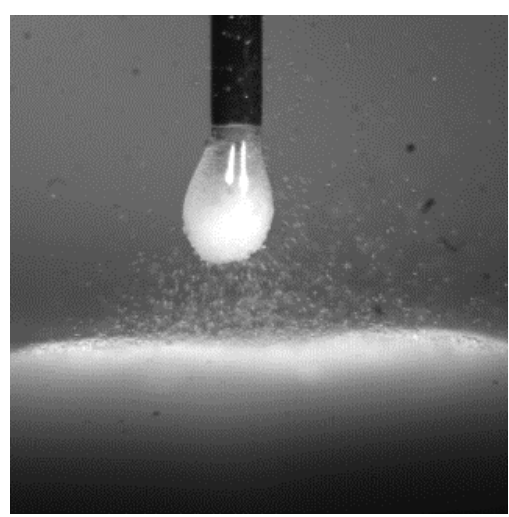

$275 \mathrm{~ms}$

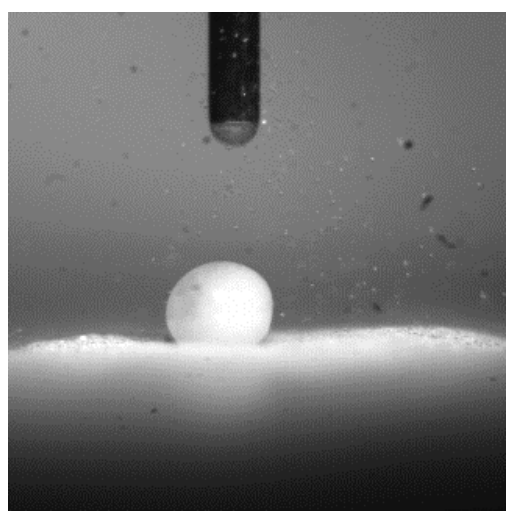

$375 \mathrm{~ms}$

Figure 7.4: The high speed video footage produced high resolution images during the particle transfers. Bed speed was $25 \mu \mathrm{m} / \mathrm{s}$. All above images are on the same scale. 
Other experiments were conducted using as received ballotini spheres $(75-90 \mu \mathrm{m})$ which exhibit some hydrophobicity. In this case full penetration into the drop did not always occur, and hence portions of spheres were observed to remain at the surface, no doubt with a contact line. Further, "pearl chain" fragments were evident at the drop surface as shown in Figure 7.5. These pearl chain structures are common when either electrostatic or magnetic forces are applied to particles.

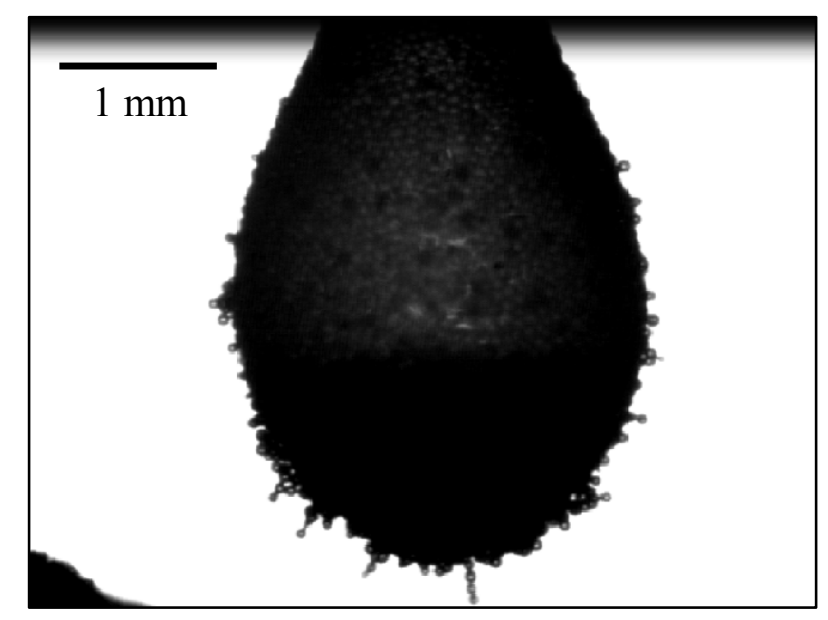

Figure 7.5: As received ballotini with the hanging water drop.

Meanwhile, cleaned rough silica (38-45 $\mu \mathrm{m})$ showed similar behaviour as 75-90 $\mu \mathrm{m}$ cleaned ballotini (with Extran). Further the same observations were seen when Milli Q water was used instead of normal tap water. When the above experiment was repeated with plastic tubing (I.D. $5 \mathrm{~mm}$ ) instead of the metal needle (I.D. $1.3 \mathrm{~mm}$ ) using cleaned glass ballotini $(75-90 \mu \mathrm{m})$, still the particles jumped in to the water drop. As a control experiment, cleaned ballotini $(75-90 \mu \mathrm{m})$ were tested with a dry needle tip without the water drop. Few particles were lifted under this scenario when the distance between the substrate and needle became $2 \mathrm{~mm}$. Thus when the needle is not conducting, the water drop behaves as a conductor and creates the electric field. Further the same experiment was conducted successfully with a drop of distilled water instead of normal water. 


\subsubsection{Hypothesis}

It is hypothesised that the Teflon was triboelectrically charged by rubbing with the Nitrile glove. Teflon has a relatively large contact work function and a high density of surface charge states, and therefore tends to acquire a strong negative charge from contacts with other materials (Wahlin \& Backstrom, 1974), although Teflon is able to impart negative charge on quartz (Manouchehri et al., 2002).

When the glass ballotini contacts with the Teflon, the ballotini becomes charged by conduction as shown in Figure 7.6. The geometry of the drop-substrate system is very similar to the much-studied sphere-plate geometry, particularly the case of a conducting sphere and an insulating charged plate (Berry, 1976; Berry \& Higginbotham, 1975). With the introduction of the drop, charge will rearrange itself on the drop surface such that its underside is positive, forming a reciprocal image of the substrate and particle bed. Collection of image charge on the bottom of the drop and possible polarisation of the bulk liquid will both affect the electric field between the drop and the substrate in a similar way. The field lines will be 'drawn into' the drop as shown in Figure 7.6 and the field magnitude under the drop will increase as it approaches the substrate. The field magnitude will also increase with height from the substrate to the tip of the drop.

The electric field between the charged substrate and the drop may have had several effects on the ballotini. The major effect is the net electrostatic force on glass ballotini. Glass is a poor electrical conductor (bulk conductivity $10^{-11}$ to $10^{-15} \mathrm{~S} / \mathrm{m}$ ), but these experiments were carried out at relative humidities of $40-50 \%$. Adsorbed water on the particle surfaces may have resulted in a much higher effective surface conductivity, and allowed the particles to also become negatively charged. The ballotini would therefore have been repelled upward, away from the Teflon. In the absence of the drop, this upward repulsion was presumably too small to counteract the gravitational force on the particles. However, as the drop approached the substrate, the concentration of the field under the drop may have become large enough to lift the particles. 
Silica, charged

by conduction

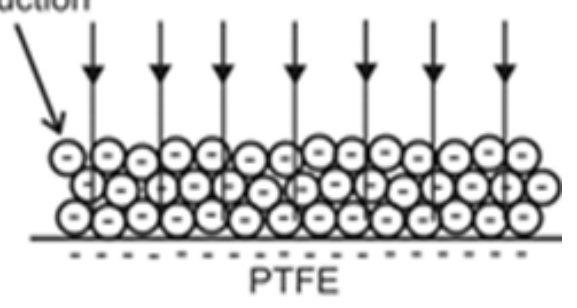

$\downarrow$
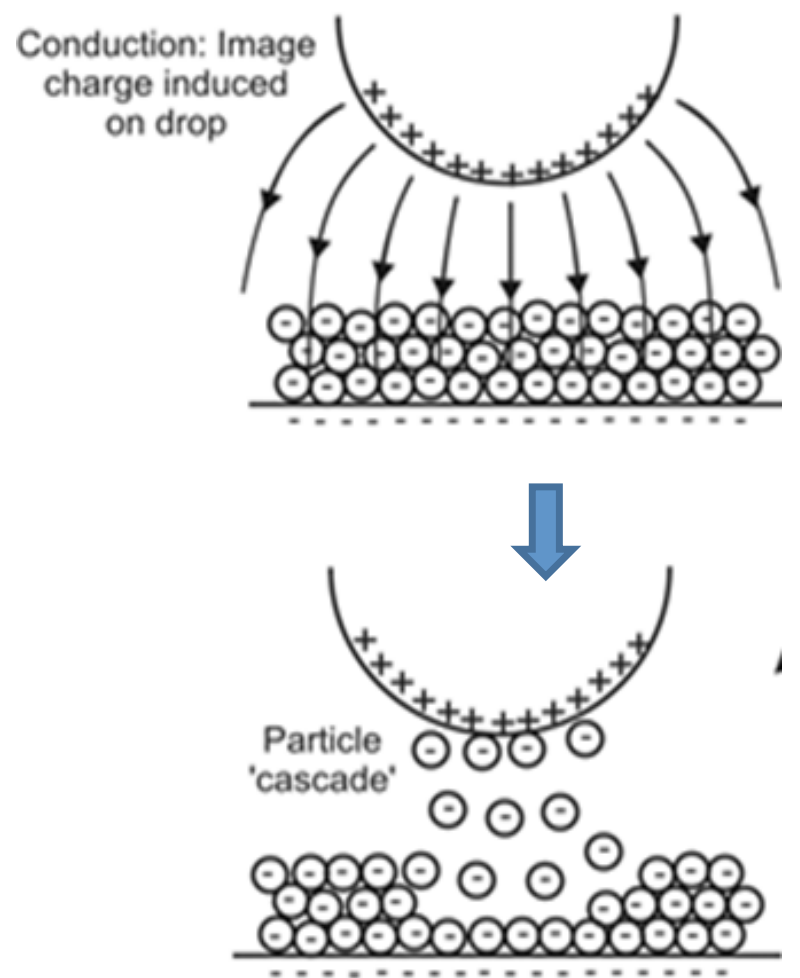

Figure 7.6: Formation of the charges and the electrical field.

Presumably, particle avalanche occurred when positive feedback mechanisms (those encouraging the growth of perturbations) predominated over negative feedback mechanisms (those that opposed perturbations) (Bechhoefer, 2005). Avalanches need not occur exclusively under the tip of the drop. In a parallel with analogous processes such as electrical discharge and brittle fracture, the avalanche depends not only on where the external field is the strongest, but also on the presence of a suitable perturbation. These issues are best illustrated by attempting to identify the main feedback mechanisms. 


\subsubsection{Positive Feedback Mechanism}

In this Section, three causes for the positive feed-back are proposed. These are particle image charge, electrostatic deformation of the drop, and particle loading.

\section{- Particle image charge:}

The charge distribution on the drop is a reciprocal image of the charge on both the substrate and the particles. Any spatial rearrangement of the charged particles will induce a corresponding rearrangement of the drop surface charge as shown in Figure 7.6. Assume an incipient cascade, consisting of a particle or particles moving incrementally closer to the drop. A reciprocal image of this incipient cascade will form on the part of the drop surface closest to those particles. The intervening electric field strength will increase accordingly, the attractive force on the closest particles will increase yet further, and the perturbation will increase.

\section{- Electrostatic deformation of the drop:}

Substantial deformations were observed for the overall drop shape during these experiments. These large-scale deformations led to elongation of the drop geometry.

Within the first 29 seconds, the drop height increased mainly due to the electric field of the substrate as shown in Figure 7.7. This initial deformation brought the underside of the drop closer to the substrate and particle bed, thus increasing the field strength immediately under the drop. This view was supported by some well controlled experiments, shown in Figure 7.7, with and without any particles on the substrate.

After 29 seconds the particles started to transfer into the drop and thereafter the drop elongated further due to the weight of the particles. The total drop height change along with the bed lifting is shown in Figure 7.7. 


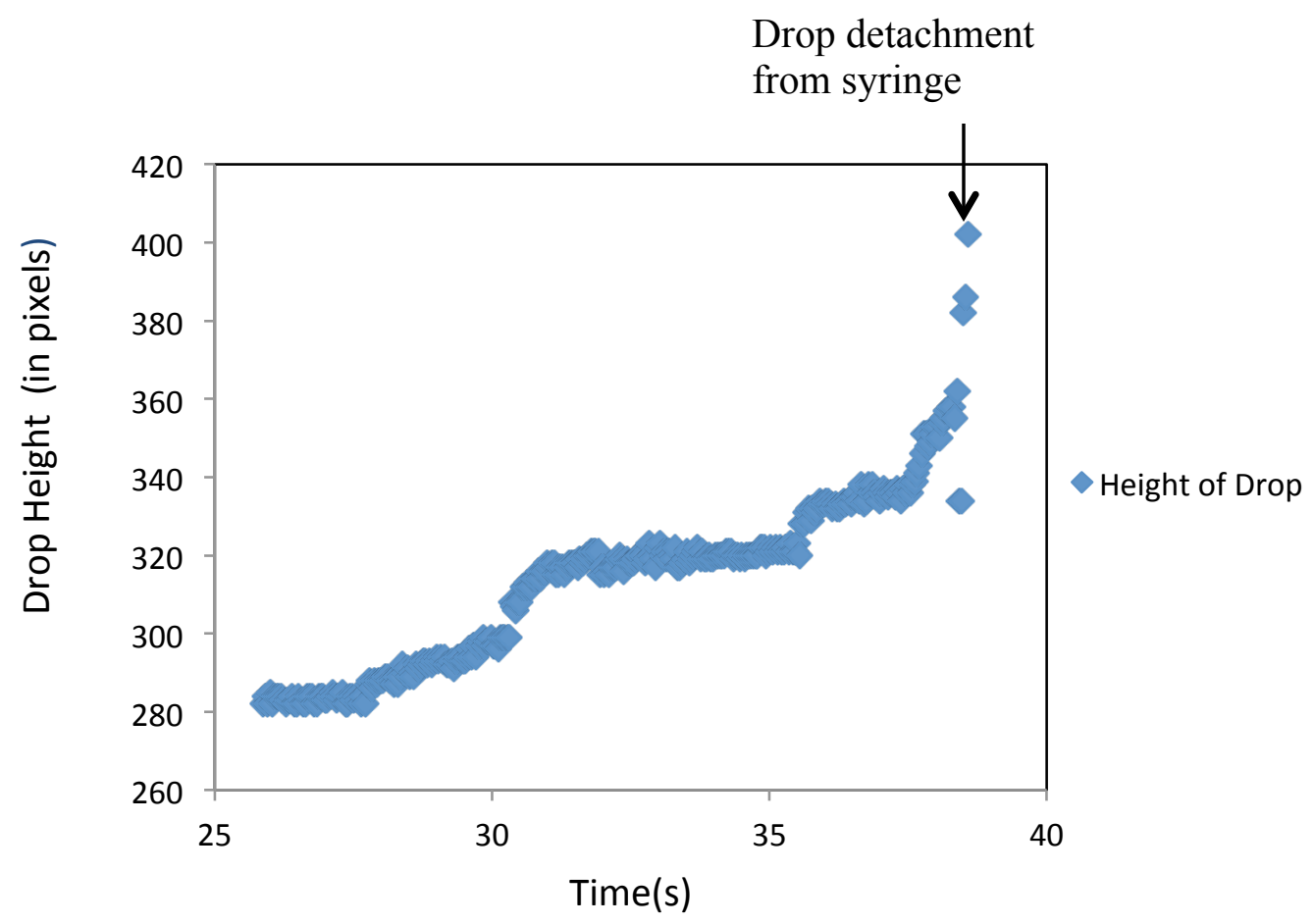

Figure 7.7: Drop height variation with the time(s). Drop height was measured from the bottom of the needle to the bottom of the drop. Particle bed lifting started after 26 s. Scale-112 pixels: $1 \mathrm{~mm}$.

The image charge concentration due to an incipient cascade, as described above, is expected to be accompanied by an additional local deformation of the drop surface in the direction of the perturbed particles. This increases the local electric field strength and thus increases the perturbation.

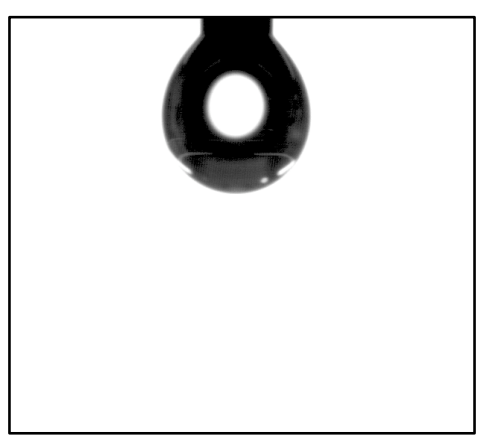

$0 \mathrm{~s}$

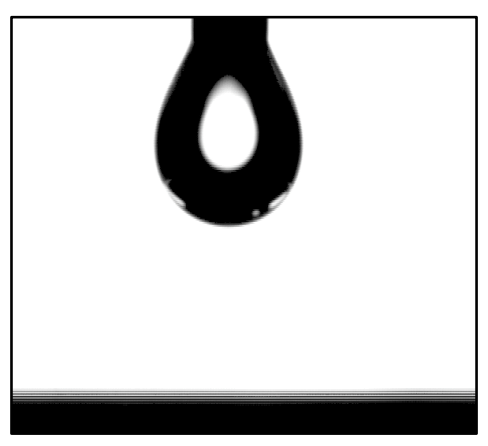

$7 \mathrm{~s}$

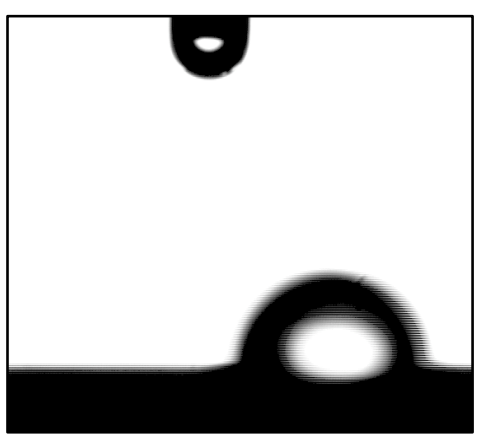

$8 \mathrm{~s}$

Figure 7.8: Drop deformations associated with the manual movement of the substrate in the absence of particles. 


\section{Particle loading:}

As particles embed in the drop, their weight deforms the drop, drawing its tip closer the substrate and particle bed. This increases the magnitude of the electric field under the drop, encouraging the transfer of further particles.

\subsubsection{Negative Feedback Mechanisms}

\section{- Charge transfer:}

As charged particles transfer to the drop, the charge on the particle bed decreases, thus decreasing the overall electric field strength. This mechanism would have a particularly strong suppressive effect on avalanches if the transferred charge persisted once the particles became embedded in the drop. However, charge measurements indicated rapid dispersal of the charge away from the particles once in contact with the drop.

\section{- Reduction of bed height:}

The transfer of particles to the drop results in an effective decrease in the height of the top of the particle bed. The formation of a 'hollow' in the top of the particle bed under the drop is illustrated in Figure 7.6. The electric field strength between the bed and the drop will decrease accordingly, discouraging further transfer of particles.

It is hypothesised that though both these feedback mechanisms are at play, the positive feedback is overcoming the negative feedback effect. It is clear that initiation of particle transfer is not simply a matter of the upward electrostatic force component exceeding the particle weight. High-speed video footage confirms that, at first, single isolated particles detach from the bed and accelerate upwards at $50-200 \mathrm{~m} / \mathrm{s}^{2}$ while the surrounding particles remain in place. This implies the bed is behaving as a cohesive mass, undergoing structural rearrangement, and sometimes releasing individual particles. Bed cohesion can be explained in terms of inter-particle friction. The force on the particles above the substrate on either side of the axis of symmetry should have a radial inward component parallel to the substrate, in addition to the upward component. 
This upward component is due to convergence of the electric field lines on the image charge of the drop (Berry \& Higginbotham, 1975). Both field components will increase as the drop-bed distance decreases. The radial inward component places the particles beneath the drop under a radial compressive stress as shown in Figure 7.9. This stress increases inter-particle friction between the spheres and results in structural locking, opposing the vertical component of the force and preventing vertical transport of the particles. As the stage is driven upward, the compressive stress increases, and the particles accordingly undergo a series of discrete stick-slip rearrangements. Some of these relieve the stress enough to release a quantity of particles. Eventually the system passes the 'tipping point' described above, when the quantity of particles transferred is so great the surface tension force can no longer support the combined weight of the drop and particles, and the downwards electrostatic force.

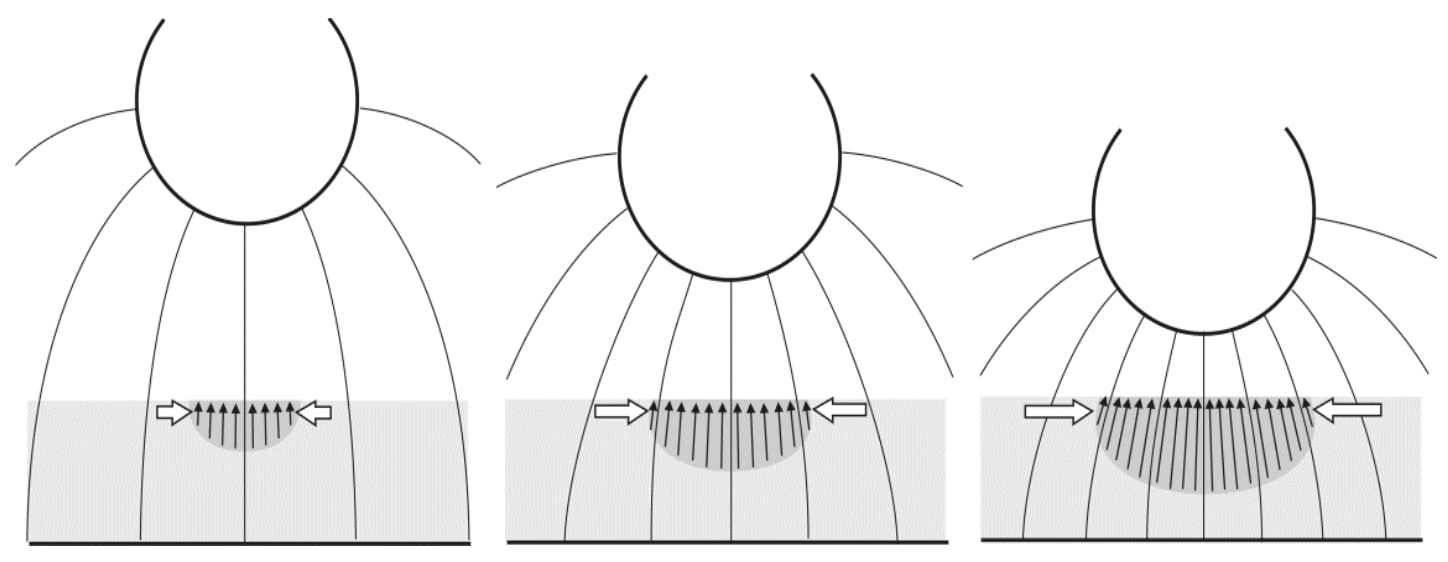

Figure 7.9: Schematic of the proposed bed cohesion mechanism. The electric field lines converge on the drop, putting the particle bed immediately under the drop under a radial compressive stress in the plane of the bed surface. This prevents the particles being gradually stripped away as the gap closes, and instead favours discrete release events associated with bed rearrangements.

As the drop and bed are brought together, electrostatic potential energy is partially converted to stress in the particle bed. Even when the system ceases to be driven, this energy can remain stored. It is hypothesised that a relatively slow rearrangement process occurs in the apparently uneventful periods between particle releases, enabling each subsequent event. If particle release does not occur, the slow rearrangement process eventually leads to a genuinely stable state, as observed. 


\subsubsection{Charge Measurements and Volume Changes with Time}

The accumulation in transferred particle charge, measured by the electrometer, reveals a strong correlation with the volume of the drop calculated using the tensiometer software. Tensiometer software calculated the drop volume by processing images of its videos. In the first instance, cumulative charge transfer was measured when the experiment was performed without particles on the substrate. The cumulative charge transferred in this case is shown in Figure 7.10. In this control experiment, the drop did not detach and the charge did not vary significantly.

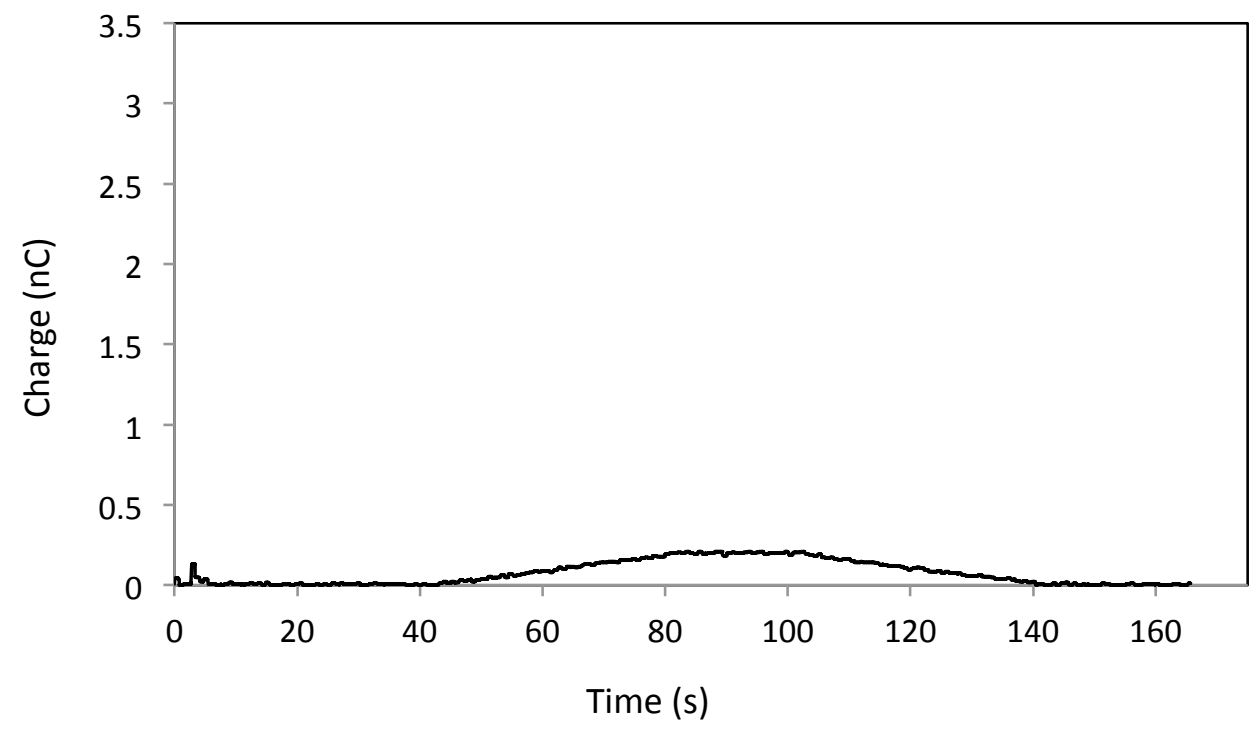

Figure 7.10: Cumulative change in the charge transferred to the drop without particles on the bed.

Figure 7.11 shows the cumulative charge transfer and volume change versus time when the same experiment was conducted with glass ballotini particles on the substrate. While the micrometre stage was driven between $\mathrm{t}=28$ and $35 \mathrm{~s}$ the total charge transfer recorded by the electrometer increased in a manner consistent with the induction of image charge on a spherical conductor approaching a negatively-charged insulating plane (Berry \& Higginbotham, 1975).

There is a rise in charge that coincides with the arrival of the charged ballotini. The charge signal rises to a plateau that represents the total charge transfer. One of the most 
striking characteristics of this system was the tendency of the particles to jump to the drop intermittently in sudden 'cascades' or 'avalanches', rather than undergoing gradual and incremental transfer as the substrate was moved toward the needle. Three large avalanches can be noted at $\mathrm{t}=45,49$ and $51 \mathrm{~s}$. This indicates that for less than some critical separation, small forward perturbations to the system tended to grow until the detachment of the drop from the needle. This scenario was punctuated by two pauses, the first from $t=46 \mathrm{~s}$ to $49 \mathrm{~s}$ and the second from $t=50 \mathrm{~s}$ to $51 \mathrm{~s}$, in which no charge transfer was observed as the lift of the substrate was paused to observe any sudden avalanches. These pauses did not lead to an avalanche possibly because the drop-bed separation was larger. However, the substrate was driven manually in this experiment using a screw micrometer stage. Once the gap was small enough for particle transfer to begin, the stage was moved in rapid advances of a few tens of microns at a time.

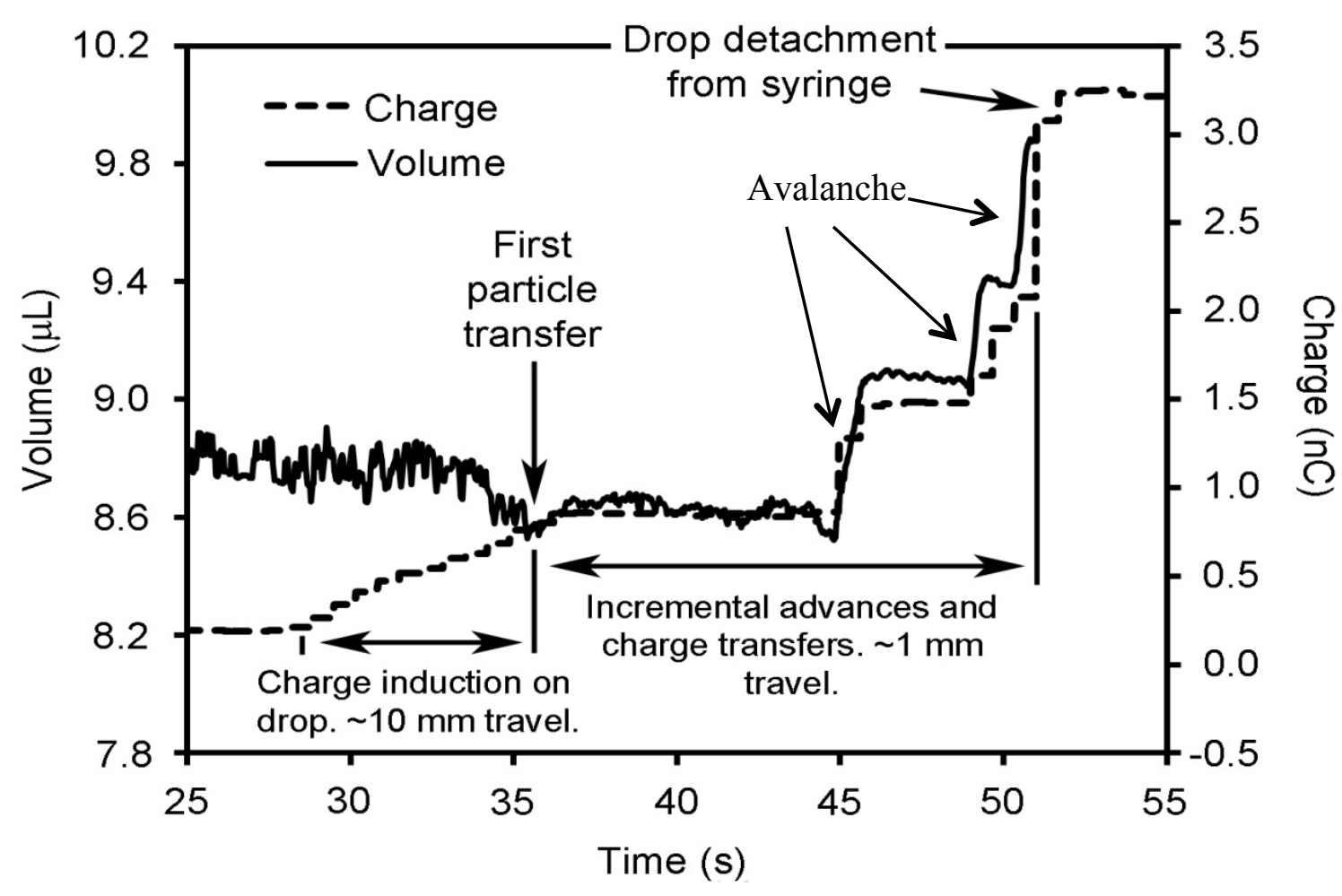

Figure 7.11: Cumulative changes in the drop volume (solid curve), corresponding to cumulative changes in the charge transfer to the drop (broken cure). Subsequent to initial particle transfer, the volume and charge changes correspond closely. Lifting of substrate started after 28 seconds. 
After the first particle transfer, volume changes followed the same behaviour as charge. With the charge increase, the volume also increased due to the drop swelling by engulfing particles. It was observed that the volume did not change when the table was paused and the particles ceased engulfing into the water drop. Although there was an initial volume decrease before the first particle transfer, as per the graph, there was a technical error of the tensiometer software in this initial volume calculation.

The effect of the moving bed was studied using a motorized stage instead of the screw micrometer in the above experiment. Figure 7.12 shows two charge-time profiles where the stage was paused after the first particles were seen to jump to the drop, but before the final avalanche.
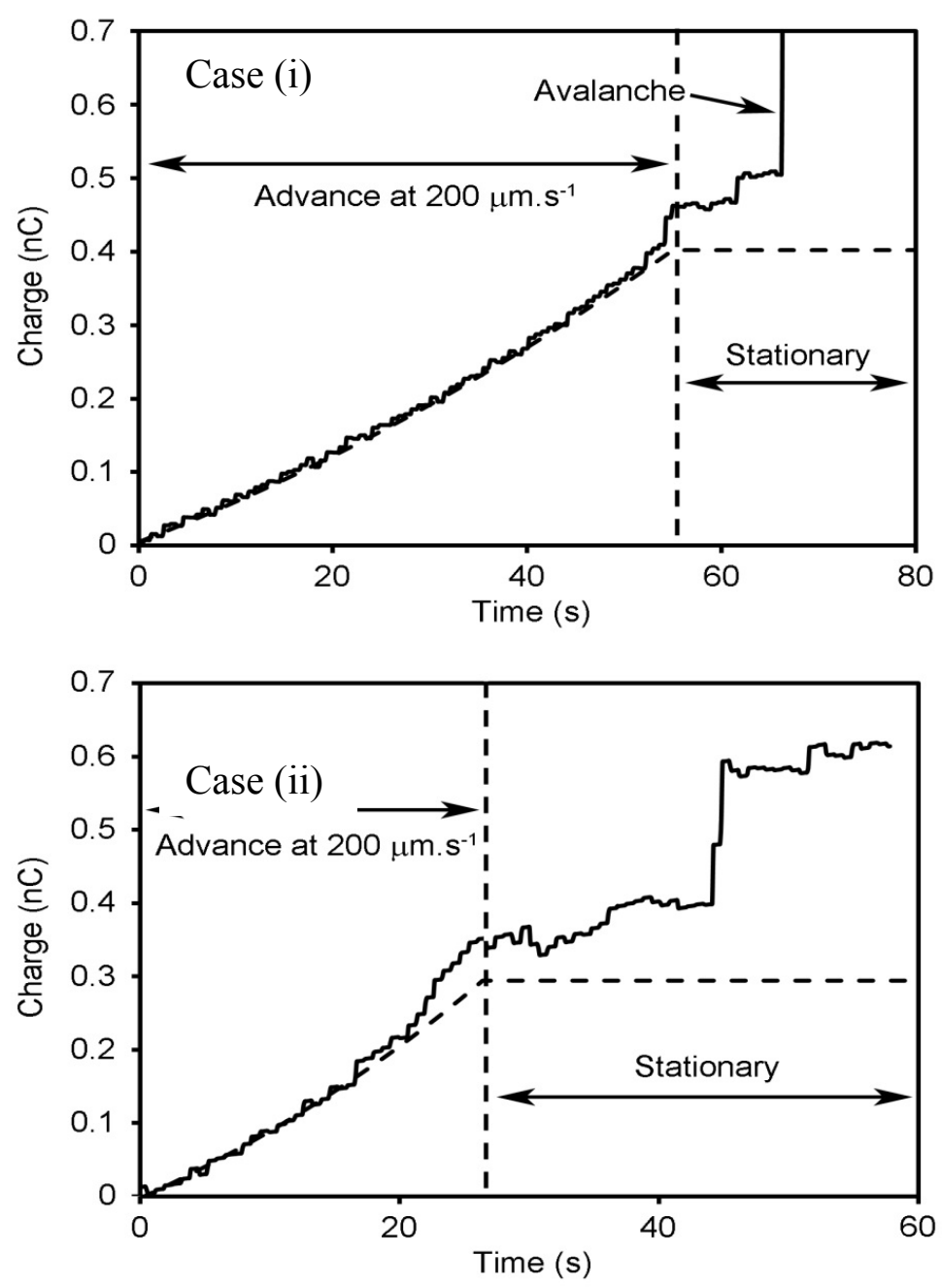

Figure 7.12: Charge transferred to the drop when the stage is advanced at a steady velocity $(200 \mu \mathrm{m} / \mathrm{s})$ then halted when particle transfer is observed. The solid curve indicates measured charge, and the broken curve indicates induction of charge on the 
drop. An avalanche occurred in the first case, but not in the second. Figure reproduced from Liyanaarachchi et al, 2013.

In case (i) a series of particle transfer events occurred spontaneously over a period of $\sim 10$ seconds, eventually leading to an avalanche and detachment of the drop. These events were punctuated by periods of a number of seconds in which no transfer was observed. In case (ii) much larger spontaneous transfer events occurred approximately 20 s after the stage was paused, but this and other smaller transfers did not lead to an avalanche, possibly because the drop-bed separation was larger when particle transfer began. These experiments demonstrate that the system does not need to be continuously driven for particle transfer to occur.

The rate of rise in the volume of the drop was measured in a series of 15 experiments and used to calculate the number rate addition of the ballotini spheres. By way of example, the volume increase in one experiment was found to be 1251 particles per second, while the electrometer showed an increase of $0.4 \mathrm{nC}$ over the same period. The ratio of these rates provided a measure of the charge, $q$, per particle, which was found to be $3.5 \times 10^{-13} \mathrm{C}$. By equating the force, $q E$, with the particle weight, $m g$, the electric field strength was estimated to be at least $21,122 \mathrm{~N} / \mathrm{C}$.

\subsubsection{Charge Measurements and Variable Speeds}

More experiments were performed with different substrate approach speeds of 25, 50, 100 and $200 \mu \mathrm{m} / \mathrm{s}$. Figure 7.13 shows the collected charge versus time profiles for these experiments. Figure 7.14 shows the same charge data plotted against the distance travelled. The avalanche points and approach curves for the experiments are much more closely grouped when plotted against distance than time, although the slope of the approach curve varies by almost a factor of two. Around the avalanche point, the rate of charge transfer neither during the pre-cascade approach phase nor in the charge transfers during the cascade did not show a systematic variation with approach speed. It should be noted that the drop size and particle bed depth were not controlled rigorously, and large variations in the tribocharge density on the Teflon substrate can be expected. 
The explosive release of particles occurred within a very narrow range of separation distance between the drop and the substrate, independent of the rate of elevation in the micrometer stage. In this and all other cases shown here, the drop fully detached from the needle well before making contact with the bed. Hence, the rapid increase in the measured value of the charge is not the result of direct conduction from the bed of silica particles to earth.

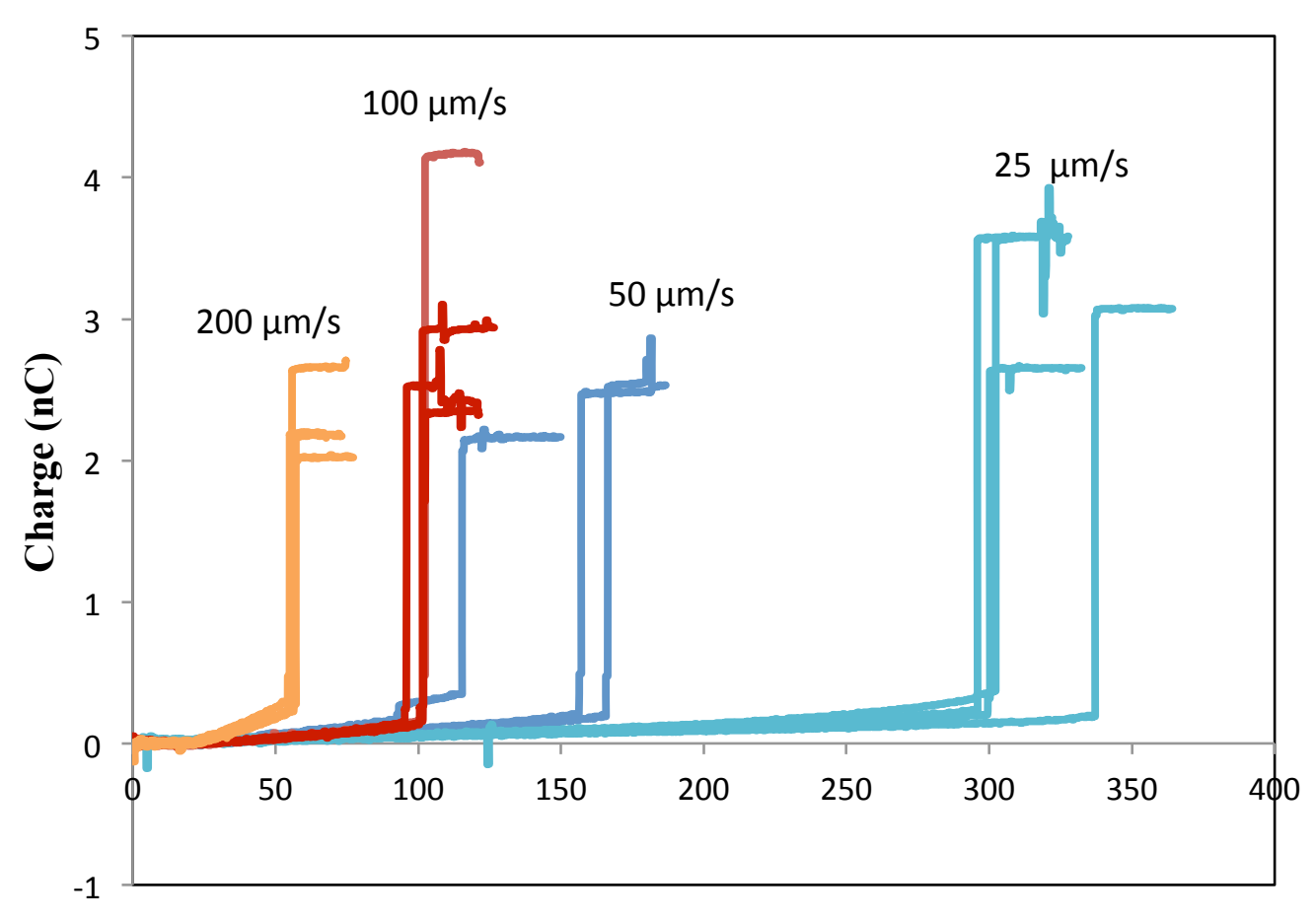

Time (s)

Figure 7.13: Charge measurements corresponding to different substrate velocities against the time. 


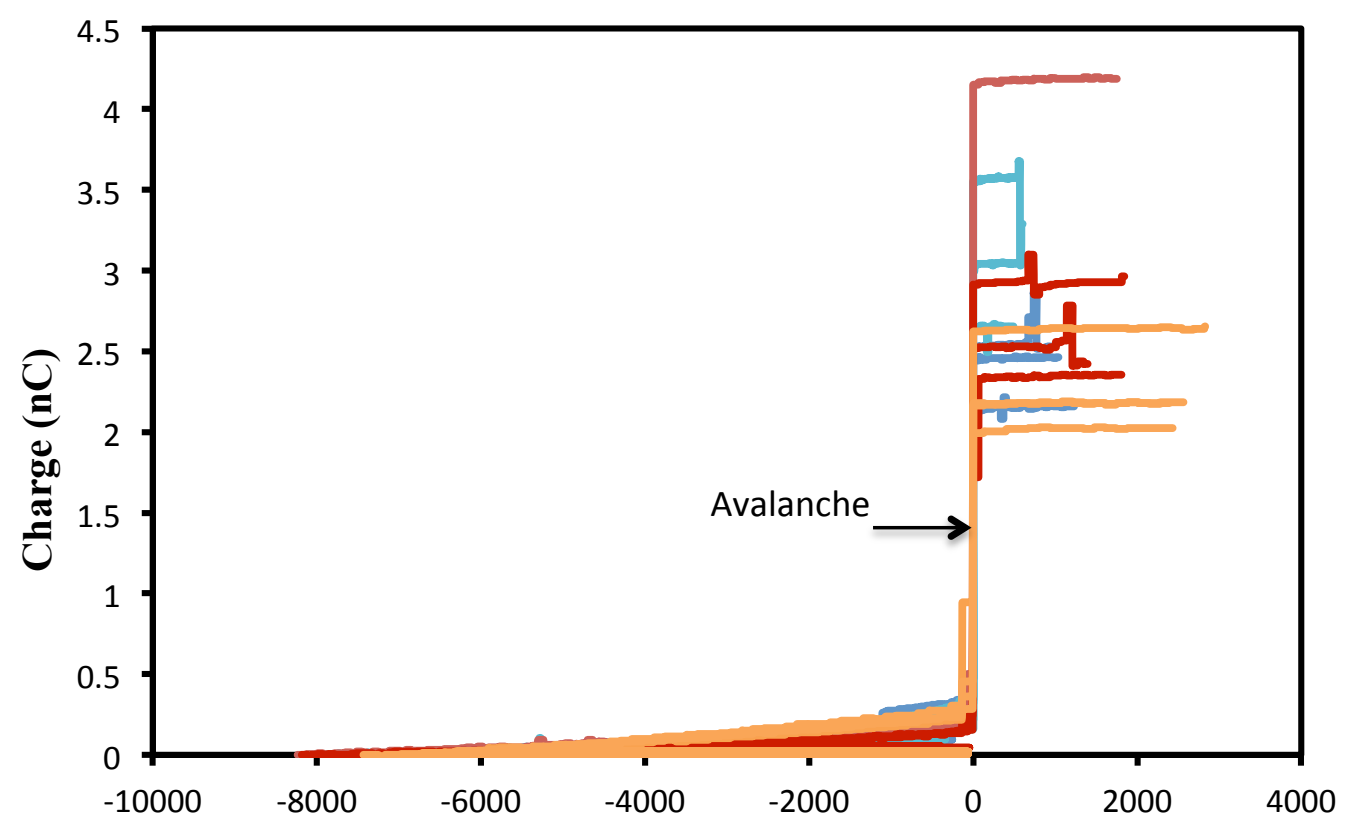

Approach displacement (microns)

Figure 7.14: Charge measurements corresponding to different substrate velocities against the approach displacements (i.e. descent time multiplied by descent speed). Zero reference was selected in each experiment where the final avalanche starts.

Figure 7.14 shows good agreement between duplicate experiments. A similar reproducibility was also achieved in the other systems studied.

\subsubsection{Hydrophilic Particle Behaviour with Different Liquid Drops}

The above mentioned phenomenon was then tested using two different liquid drops. The same experiment was repeated with hydrophilic glycerine and hydrophobic hexane using cleaned glass ballotini $(75-90 \mu \mathrm{m})$.

\subsubsection{Glass Ballotini with Glycerine}

Glycerine (glycerol, $\mathrm{C}_{3} \mathrm{H}_{8} \mathrm{O}_{3}$ ) is a colourless, hydrophilic, viscous liquid with three hydroxyl (-OH) groups. Glycerine is highly soluble in water and hygroscopic in nature. Being hygroscopic, it is able to absorb water from the atmosphere. Glycerine is not a good conductor and its conductivity is around $0.1 \mu \mathrm{S} / \mathrm{cm}$ at $20{ }^{\circ} \mathrm{C}$ (Gamota et al., 2004; 
Marchand, 2012). This is barely $0.2 \%$ of the value for tap water (Table 7.2), so it might be expected that phenomena that relied on liquid conductivity would be significantly reduced.

However, when glycerine was used, it was observed that hydrophilic glass ballotini lift from the bed and engulfed into the drop in the same way they behaved with water drops. The image sequence of this experiment is given in Figure 7.15. It was observed that the particles jumped into the drop at the top as well as at the bottom. A few avalanches were also observed with glycerine. This proved that an electric field exists, even when very poorly conducting liquids are used.

The glass ballotini covered the outer surface of the glycerine drop for a long time, without penetrating in to the drop. This may be due to the high viscosity of glycerine. In addition, a number of pearl chains were also formed on the surface. In Images 1 and 2 of Figure 7.15, streaks caused by fast-moving particles from bed were also observed. With further approach of the bed, more and more particles jumped towards the glycerine drop and formed a thick layer of glass ballotini around the outer surface of the drop as shown in Image 5 and 6. 


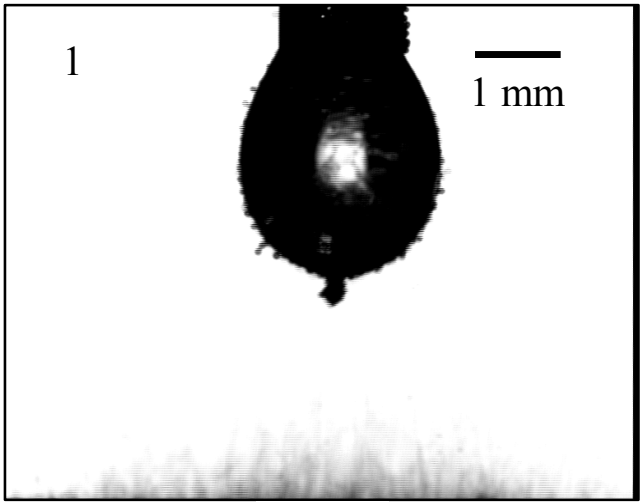

$13 \mathrm{~s}$

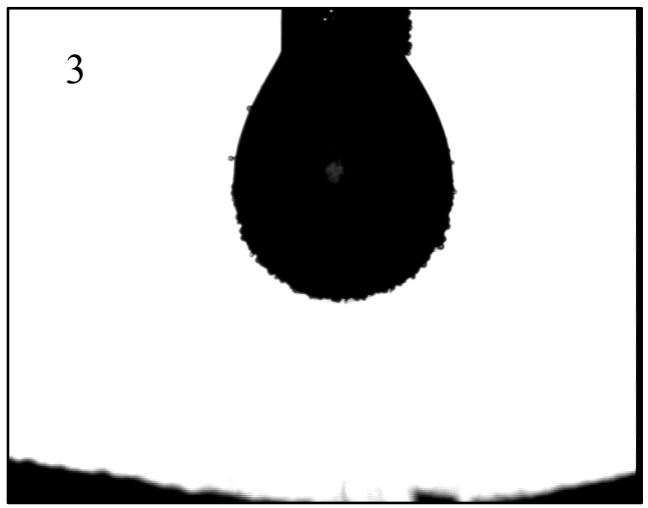

$13 \mathrm{~s}$

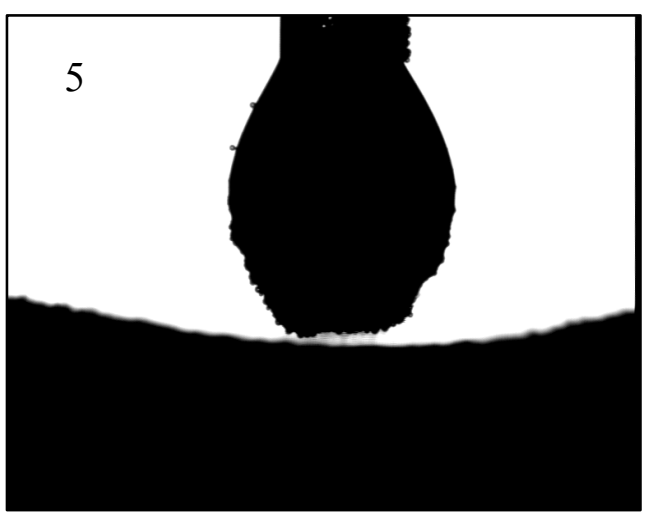

$26 \mathrm{~s}$

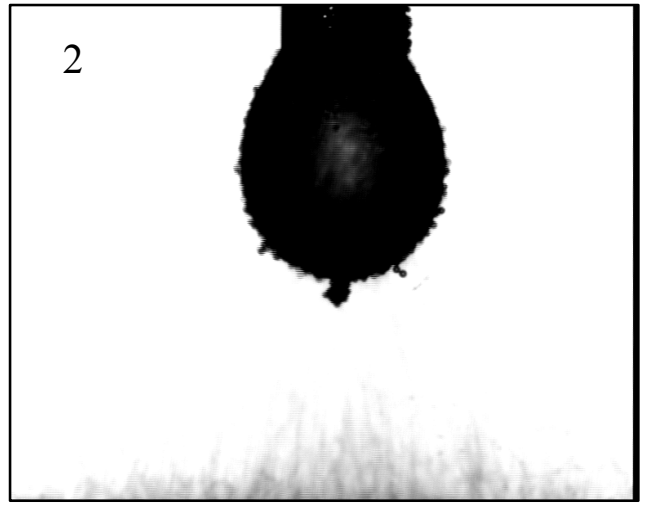

$13 \mathrm{~s}$

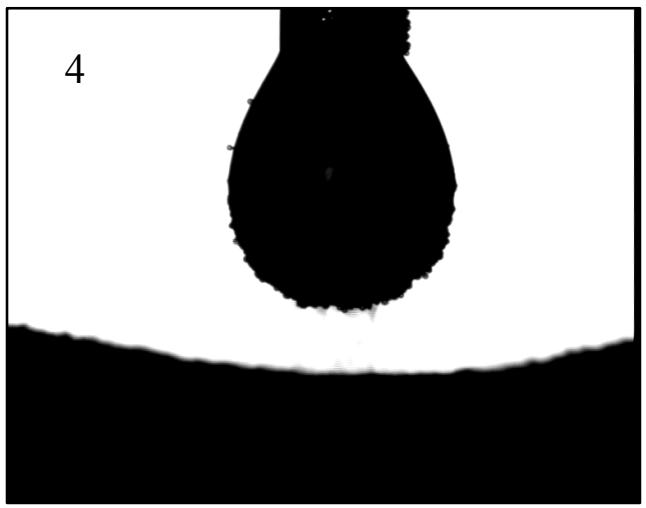

$18 \mathrm{~s}$

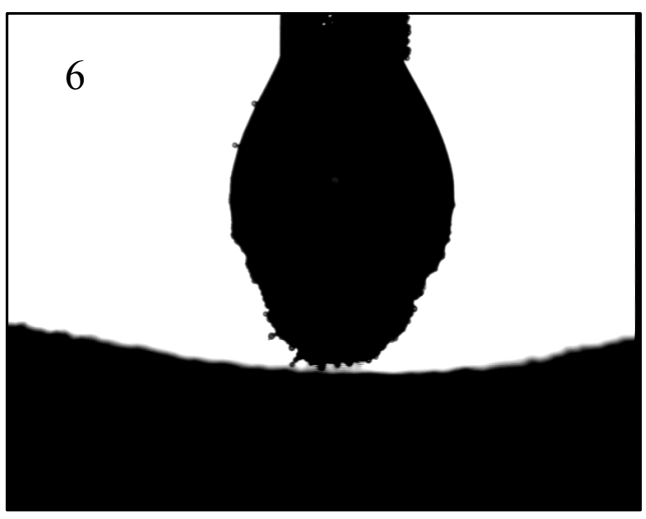

$32 \mathrm{~s}$

Figure 7.15: Image sequence of interaction between a bed of glass ballotini and a hanging drop of glycerine. All above images are on the same scale.

\subsubsection{Glass Ballotini with Hexane}

Hexane $\left(\mathrm{C}_{6} \mathrm{H}_{14}\right)$ is a pure hydrocarbon and a non-polar liquid. Thus hexane also behaves as a non-conductor (Mulligan, 2003). Hexane has a very low dielectric constant (1.88 at $25^{\circ} \mathrm{C}$ ) compared to polar liquids such as glycerine with a dielectric constant of a 42.5 at 
$25{ }^{\circ} \mathrm{C}$ (Lechner \& Wohlfarth, 2008). It was difficult to obtain a proper hanging drop of hexane due to its low surface tension. The surface tension of hexane is $18 \mathrm{mN} / \mathrm{m}$ compared to $72.8 \mathrm{mN} / \mathrm{m}$ for water (Table 7.2 ).

A deformation of the drop was observed at the initial stage of particle (ballotini) jumping into the drop when hydrophobic Hexane was used as the liquid despite of the poor conductivity of hexane. Avalanches were also observed with non-conducting hexane as shown in Figure 7.16.
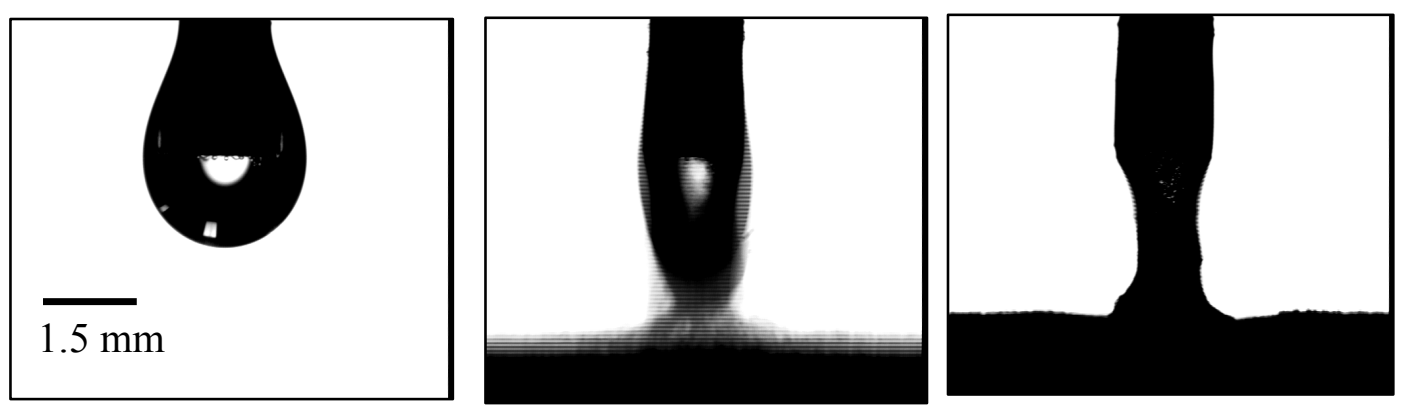

Figure 7.16: Image sequence of interaction between a bed of glass ballotini and a hanging drop of hydrophobic hexane. All above images are on the same scale.

\subsubsection{Hydrophobic Particle Behaviour with Water Drops}

Hydrophobic particles such as coal $(38-45 \mu \mathrm{m}, 63-75 \mu \mathrm{m}, 75-90 \mu \mathrm{m})$ and polyvinyl chloride (PVC) particles $(106-125 \mu \mathrm{m})$ were transferred to water drops. Figure 7.16 shows the image sequence of PVC particles interactions with the hanging water drop.

Although the avalanches were observed with the PVC particles, they did not penetrate in to the water drop. Instead they deformed the drop and finally detached it from the needle due to the high density of particles. This proved that PVC particles also obtained the negative charges through conduction of the rubbed Teflon. 


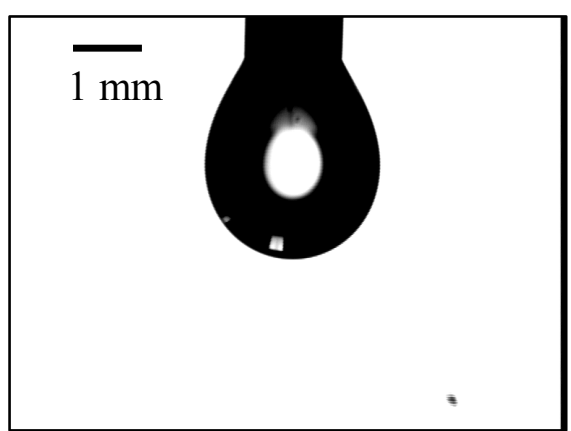

$9 \mathrm{~s}$

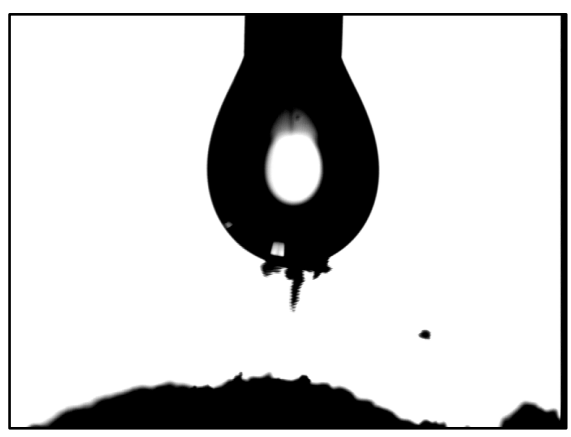

$10 \mathrm{~s}$

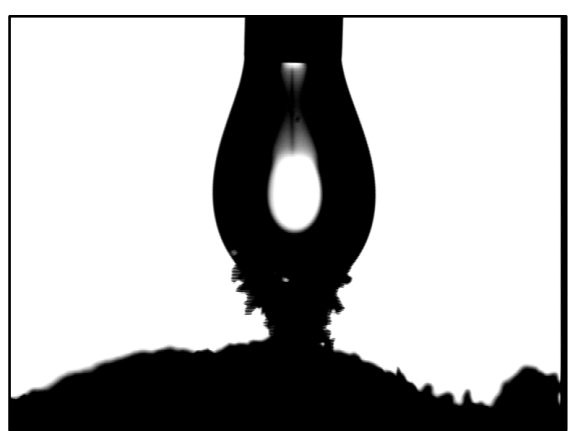

$11 \mathrm{~s}$

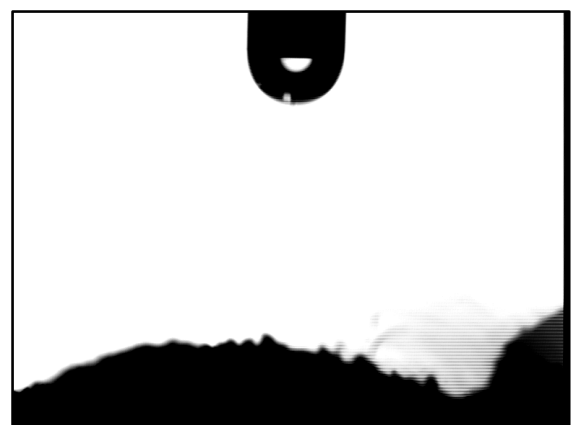

$11 \mathrm{~s}$

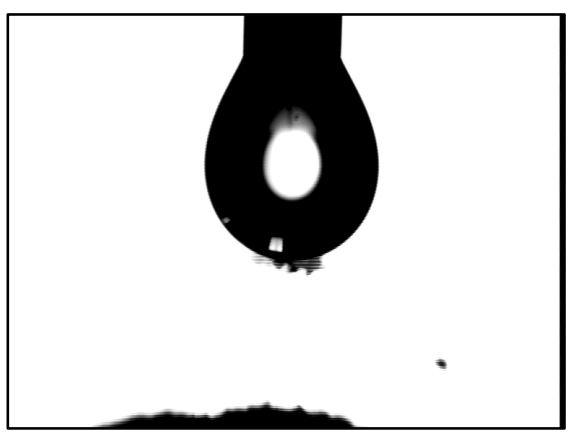

$10 \mathrm{~s}$

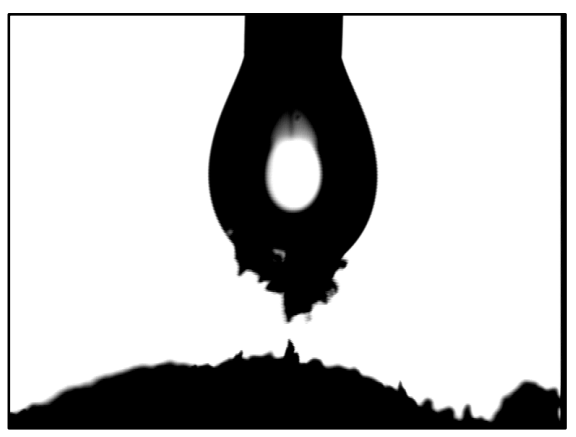

$10 \mathrm{~s}$

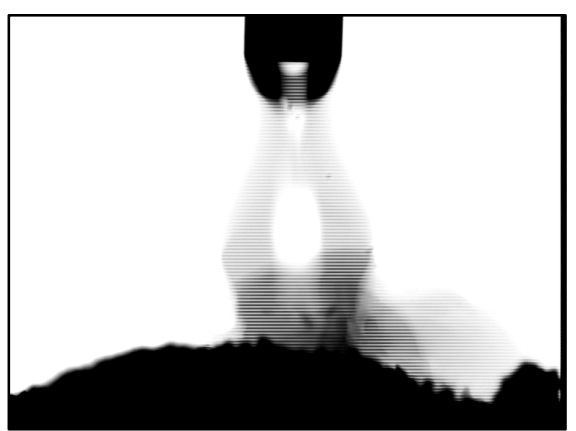

$11 \mathrm{~s}$

Figure 7.17: Image sequence of interaction between a bed of $\mathrm{PVC}$ particles and a hanging water drop. The bed was driven manually. All above images are on the same scale. 
When coal particles were used as the bed material, regardless of the size range, they jumped to the outer surface of the drop. Initially coal particles attached to the bottom of the drop and then branched out towards the top of the drop against gravity. Coarser particles $(75-90 \mu \mathrm{m})$ formed clear branches and caused less deformation of the drop compared to finer particles as shown in Figure 7.18. Finer coal particles spread out and formed a packed domain at the surface of the drop much faster than coarser particles.
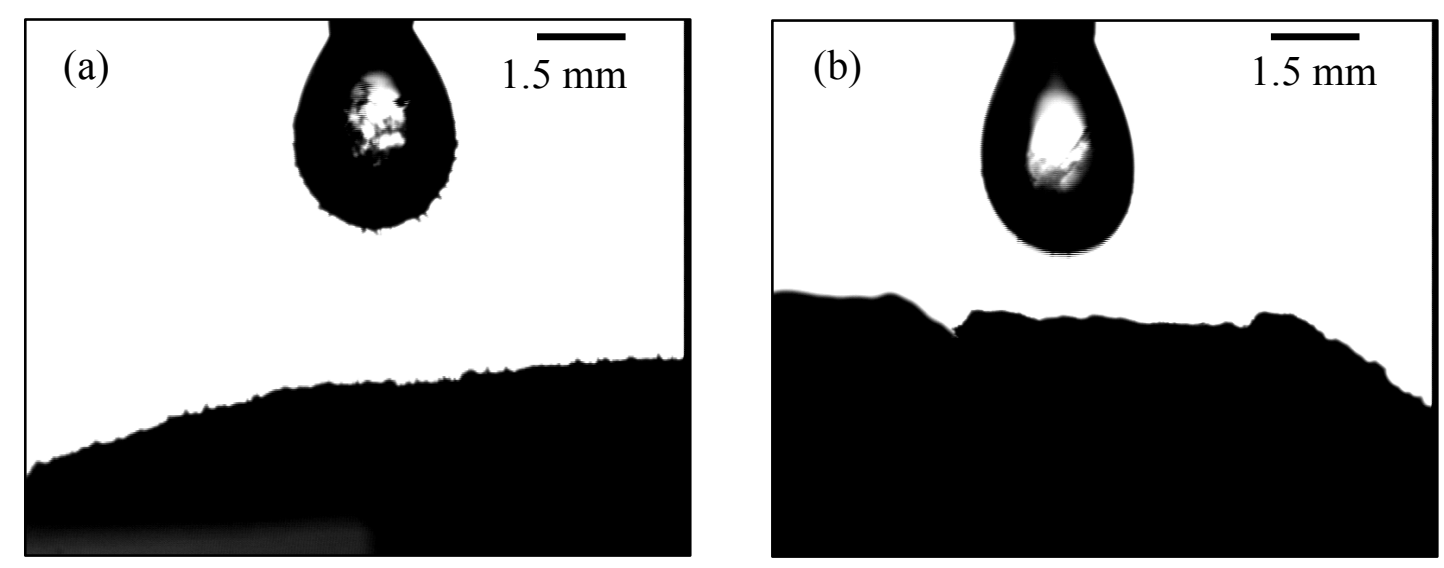

Figure 7.18: (a) Coal 75-90 $\mu \mathrm{m}$ particles show a clear branching and less deformation compared to (b) coal $38-45 \mu \mathrm{m}$ particles.

After covering up the whole surface of the drop, these were similar to the 'liquid marbles' that are formed when a drop rolls across the bed as discussed in Section 3.4.4. This packed layer appears to stabilize the drop surface and make it rigid. As a result, much less deformation is observed, which tends to suppress 'avalanches'. In general, the system exhibits a more even and gradual particle transfer over a larger vertical distance than it does for hydrophilic particles.

\subsubsection{Hydrophilic and Hydrophobic Particle Mixtures with Water Drops}

Different sizes and different proportions of coal silica mixtures were also used as the bed material to investigate the particle behaviour with water drops. Two size fractions of coal and silica were used in these experiments. They are 38-45 $\mu \mathrm{m}$ and 75-90 $\mu \mathrm{m}$. Three different composition mixtures were prepared from each size range of these two components. 


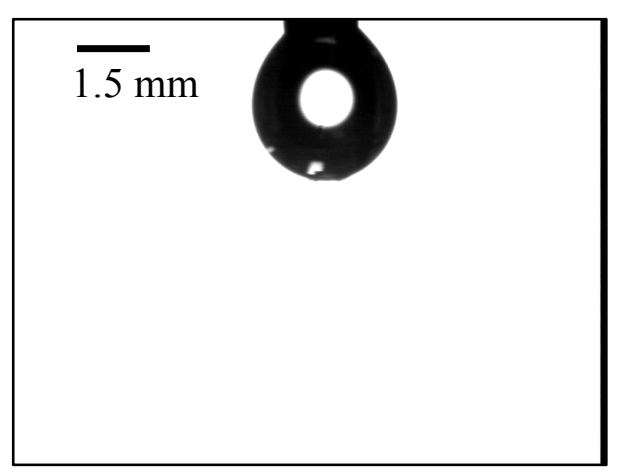

$14 \mathrm{~s}$

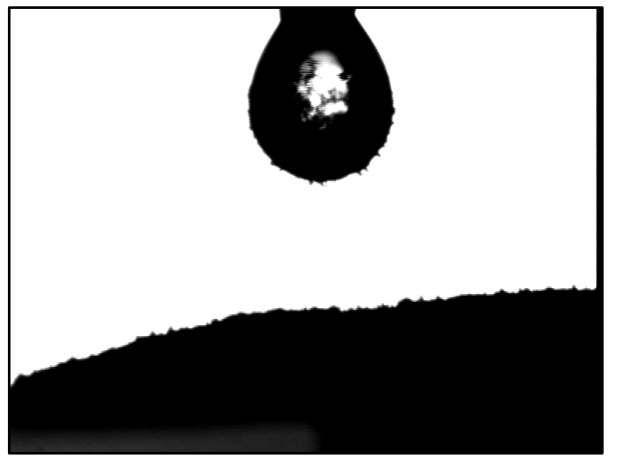

$18 \mathrm{~s}$

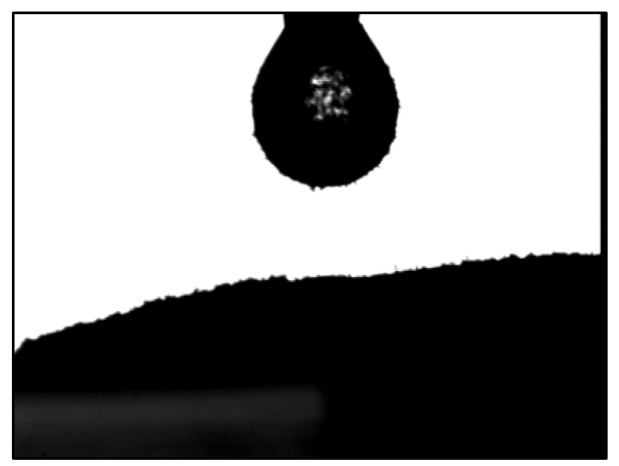

$19 \mathrm{~s}$

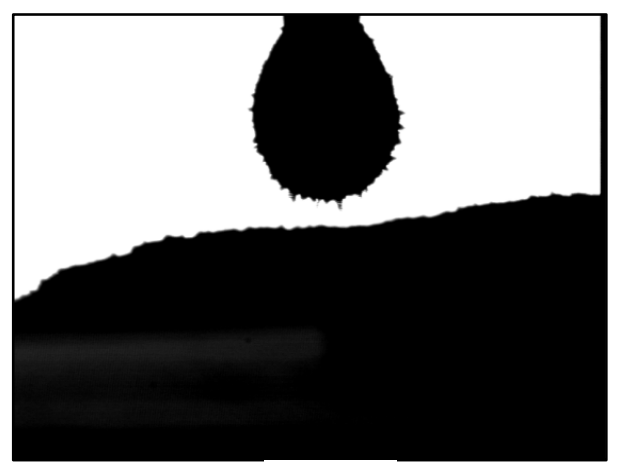

$38 \mathrm{~s}$

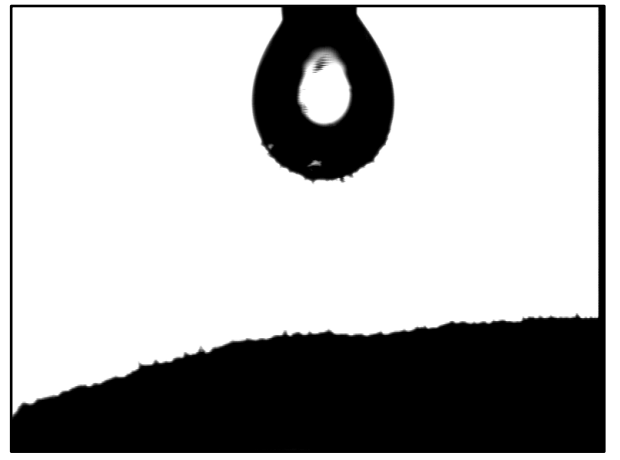

$16 \mathrm{~s}$

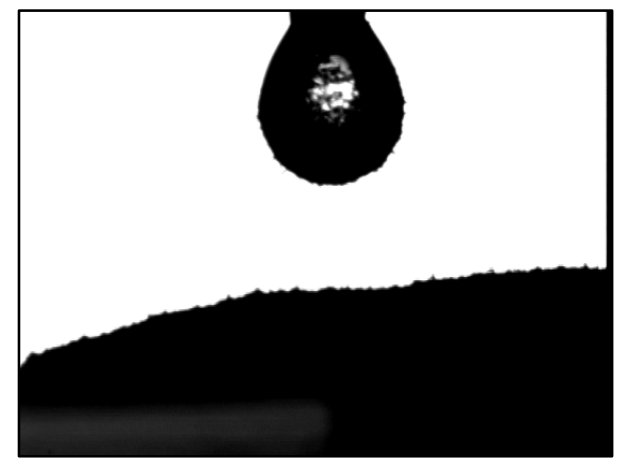

$18 \mathrm{~s}$

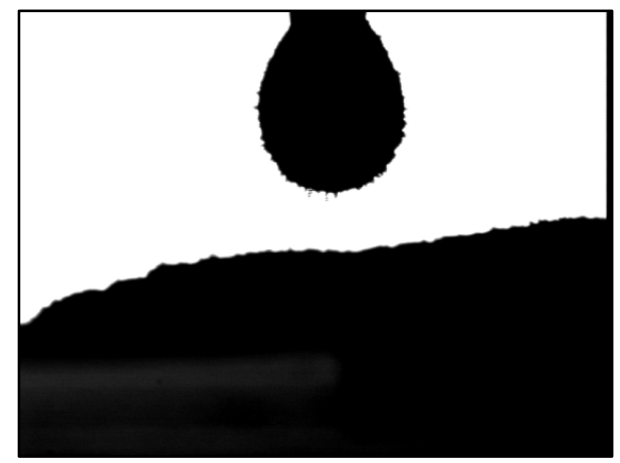

$21 \mathrm{~s}$

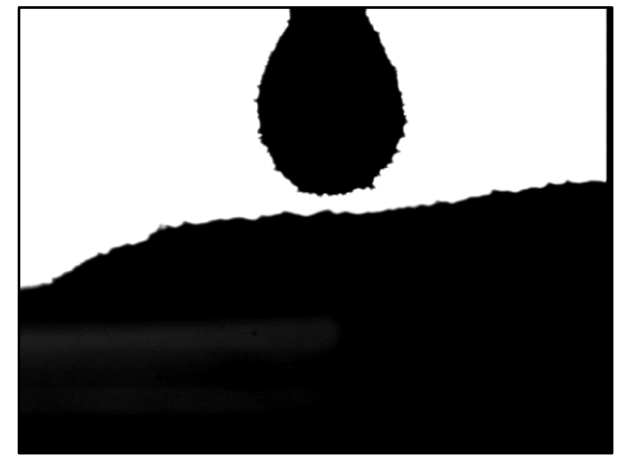

$59 \mathrm{~s}$

Figure 7.19: Image sequence of interaction between a bed of coal $(90 \% \mathrm{w} / \mathrm{w})$ and silica $(10 \% \mathrm{w} / \mathrm{w})$ particles in $75-90$ micron size range with a hanging water drop. Bed was driven manually. All above images are on the same scale. 
Silica $90 \% \mathrm{w} / \mathrm{w}$ with coal $10 \% \mathrm{w} / \mathrm{w}$, silica $50 \% \mathrm{w} / \mathrm{w}$ with coal $50 \% \mathrm{w} / \mathrm{w}$ and silica $10 \%$ w/w with coal 90\% w/w. Figure 7.19 shows the image sequence of behaviour of coal $(90 \% \mathrm{w} / \mathrm{w})$ and silica $(10 \% \mathrm{w} / \mathrm{w})$ mixture in $75-90 \mu \mathrm{m}$ size range with a hanging water drop.

For both size fractions, it was observed that silica particles were engulfed by the water drop whereas the coal particles formed layers packed around the drop. Once a coal layer had formed, it hindered the engulfment of further silica and stabilised the drop. A few more silica particles were slowly engulfed, but only after being attached to the outer surface for more than a couple of seconds. It was observed that the engulfing rate of glass ballotini decreased with the time. The coal layer tended to stabilize the drop. Hence, the detachment of the drop from the needle was delayed by more than a minute. But the interactions with the bed of particles decreased with time.

However, fine (38-45 $\mu \mathrm{m})$ particle mixtures jumped to the water drop much faster than the coarser $(75-90 \mu \mathrm{m})$ particle mixtures. With $90 \% \mathrm{w} / \mathrm{w}$ silica and $(50 \% \mathrm{w} / \mathrm{w})$ coal silica bed experiments more silica particles were engulfed into the drop compared to $90 \% \mathrm{w} / \mathrm{w}$ coal case in both size ranges. Avalanches were also observed with all three mixtures of both size ranges. The drop did not detach from the needle in any of these experiments covering the 75-90 $\mu \mathrm{m}$ range. However, with fine fraction $(38-45 \mu \mathrm{m}$ range) mixtures, the drop detached for the $90 \% \mathrm{w} / \mathrm{w}$ silica- $10 \%$ coal and $(50 \% \mathrm{w} / \mathrm{w})$ coal silica bed experiments.

\subsection{Conclusions}

This chapter reports on new developments related to the field of electrostatics. Since there has been very little direct research into electrostatic charges in this context, preliminary experiments were carried out to identify the phenomena that occur. Extreme care and well planned experiments were required to reproduce the results. The isolation of the charge produced from rubbing the Teflon was crucial in these experiments. There were numerous causes of charge loss.

The particles were observed to follow the field lines in these experiments. The growth of perturbations, which leads to avalanches was studied in terms of the characteristics of 
the system - the substrate-drop distance, the substrate speed, and the charge transferred by the particles. Charge transfer was independent of the substrate velocity and closely related to the displacements of the substrate.

When the behaviour of different liquids was tested it was shown that the conductivity of the liquid does not need to be significant for particles to be engulfed. Hydrophobic particles in a wide size range $(38-90 \mu \mathrm{m})$ also transferred to the outer surface of the water drop. However, hydrophilic silica and hydrophobic coal particle mixtures displayed a different behaviour with the hanging drop. These particles also jumped toward the drop, but hindered the drop detaching from the needle. However, further studies are required to analyse the particle mixture behaviours with the hanging water drop. It would be worthwhile to study the behaviour of different size and compositions of hydrophobic and hydrophilic mixtures such as coal 75-90 $\mu \mathrm{m}$ with silica 38-45 $\mu \mathrm{m}$. 


\section{Chapter 8}

\section{CONCLUSIONS AND RECOMMENDATIONS}

8.1 Conclusions

8.2 Recommendations 


\subsection{Conclusions}

In this study two novel approaches were investigated for the selective collection of fine hydrophilic and hydrophobic particles (less than $100 \mu \mathrm{m}$ ) using water drops. The first approach used the flow of air to drive a dispersion of particles through a system of falling water drops, the goal being to collect the hydrophilic particles, while ideally allowing the hydrophobic particles to pass through the system in a dry form. A mathematical model was also developed to identify the key variables influencing the system. The second approach used electrostatic forces to transport the particles towards a given drop. In this case the work focussed on establishing the factors that needed to be controlled in order to ensure reproducibility.

The first approach required the development of a new laboratory scale experimental system to study the gas-particle interaction. This development required a number of modifications in order to achieve this major objective of the research, resulting in the establishment of a novel and reliable laboratory scale experimental system. The system consisted of a feed column, water distribution chamber mounted on a collision chamber, and an air suction pump. Fine water drops were formed and permitted to settle downwards through the dispersion of particles. Hydrophilic and hydrophobic particles covering a broad size range were used to investigate their relative collection efficiency.

An analytical model was also developed to predict the recovery of particles by the drops. This model was developed using two asymptotic conditions, one in which the particle speed was much higher than the drop speed, and one in which the particle speed was negligible compared to the drop speed. A Discrete Element Method (DEM) simulation was also used in order to assess the analytical model, in both extremes, and within those extremes. Excellent agreement was achieved between $U_{p} \gg U_{d}$ extreme, and hence it was concluded that the model was a good first order description. In particular, the analytical model provided an appreciation of the key parameters responsible for insuring a given level of collection. The model showed that the particle recovery is proportional to the water flux and active length of the collision zone. The recovery is also inversely proportional to the diameter of the drops and the velocity of the fastest species, either the particles or the drops. 
The experimental work demonstrated a linear correlation between the recovery of the particles in the underflow with increasing water flux up to $0.0167 \mathrm{~m}^{3} /\left(\mathrm{m}^{2} \mathrm{~s}\right)$. Spherical glass ballotini, as received, and spherical ballotini treated with Extran ${ }^{\circledR}$, were used in the initial experiments (size range 23-47 $\mu \mathrm{m}$ ). By cleaning the particles with Extran, it was possible to achieve a slight change in surface properties, making the particles totally hydrophilic. The cleaned particles showed a 7\% increase in recovery. The recovery then decreased by $7 \%$ when the particle size decreased from 23-47 $\mu \mathrm{m}\left(d_{50}=33\right.$ $\mu \mathrm{m})$ to $17-36 \mu \mathrm{m}\left(d_{50}=25 \mu \mathrm{m}\right)$. This reduction was attributed to the lower inertia of the finer particles. Interestingly, irregular shaped hydrophilic silica particles did not show the same sensitivity to variations in particle size. Similarly, the recovery of irregular shaped hydrophobic coal particles did not vary with particle size. However, the recovery was $10 \%$ lower than the recovery of the hydrophilic silica. Indeed the selectivity between the hydrophilic silica and the hydrophobic coal was found to be 1.5. Moreover, the selectivity ratio between the silica and coal in a mixed state increased to 2.5. This increase was attributed to a blocking mechanism. Increasing the air flow rate (velocity) caused a slight reduction in particle recovery.

The second approach involved the use of the electrostatic force to transport the particles towards the drop surface. In these experiments, it was observed that the electrostatic forces could lift particles across a gap of several millimetres $(5 \mathrm{~mm})$. The system exhibited the gradual release of fine particles from the lower substrate, followed by an almost explosive release of particles beyond a critical tipping point. The electric field strength was estimated by measuring the charge transfer, the volume of particles collected, and hence the charge per particle. The factors responsible for the reproducibility were studied in detail. Very minor issues, such as the spillage of a few particles across the Teflon substrate and onto the lower glass substrate was sufficient to produce charge release, and hence failure of the experiment. The distance between the substrate and the drop was also found to be more important in affecting particle lift than the substrate speed. While most of the experiments involved the use of glass ballotini, the work was extended to include a much broader range of particles, including coal. 


\subsection{Recommendations}

The main aim of this research was to establish the potential for selective collection of fine hydrophilic particles using water drops. Although the work was successful in quantifying the selectivity based on differences in hydrophilicity / hydrophobicity, the effect was insufficient to justify the development of a new separation technology under the experimental conditions examined here. The major problem was that hydrophobic particles tend to attach to the outer surfaces of the drops. Further, ultrafine coal particles have the potential to saturate the surface and prevent the engulfment of the hydrophilic silica particles. Moreover, the hydrophilic particles may become contaminated by the ultrafine hydrophobic particles. Thus, the potential for taking this work further may be limited, from an applications perspective.

The experimental work on electrostatic forces revealed a complex and intriguing phenomenon. The major advance here was the establishment of the rules needed to ensure reproducibility. This work, which resulted from serendipity, offers the potential for developing a new program of experimental work. Well controlled and very effective particle transport was achieved using the electrostatic forces. This approach offers an alternative method for forming so-called liquid marbles, with several attendant advantages. For example, liquid marbles could be made with a combination of hydrophilic and hydrophobic particles by this method. It would be interesting to further study the behaviour of non-conducting liquids such as hexane in this context. Further, there is a considerable scope to study the behaviour of hydrophobic polymer particles and hydrophilic liquid marbles as drug and pharmaceutical powders. 


\section{REFERENCES}

Adamson, A.W. \& Gast, A.P. (1997), Physical chemistry of surfaces, $6^{\text {th }}$ ed., p.7.

Ahmed, N. \& Jameson, G. (1989), Flotation Kinetics, Mineral Processing and Extractive Metallurgy Review, $51(1 \& 4)$, p.77-99.

Aplan, F.F. (1979), Flotation. Kirk-Othmer Encyclopedia of Chemical Technology, 10, p. 523-547.

Asthana R, \& Sobczak N. (2000), Wettability, Spreading, and Interfacial Phenomena in High-Temperature Coatings, JOM-e, Minerals, Metals \& Materials Society, 52(1). http://www.tms.org/pubs/journals/JOM/0001/Asthana/Asthana-0001.html

Aussillous, P. \& Quéré, D. (2001), Liquid marbles, Nature, 411(6840), p.924-927.

Aussillous, P. \& Quéré, D. (2006), Properties of liquid marbles, Proceedings of the Royal Society A: Mathematical, Physical and Engineering Science, 462(2067), p.973999.

Bailey, A.G. (2001), The charging of insulator surfaces, Journal of Electrostatics, 51(52), p.82-90.

Bechhoefer, J. (2005), Feedback for physicists: A tutorial essay on control. Reviews of Modern Physics, 77, p.783-836.

Bennett, A.J.R., Chapman, W.R., \& Dell, C.C. (1958), Froth and Flotation of Coal, $3^{\text {rd }}$ International Coal Preparation Congress, p.452-462.

Berry, J.S. (1976), Electrostatic forces on a conducting sphere due to a charge on a dielectric half-space. Journal of Physics A: Mathematical and General Physics, 9, p.1939-1945.

Berry, J.S. \& Higginbotham, I.G. (1975) Electrostatic forces on a conducting sphere due to a charged, insulating plane. Journal of Physics A: Mathematical and General Physics 8, p.1842-1851.

Bertrand E., Blake, T.D \& Coninck, J.D. (2009),Influence of solid-liquid interactions on dynamic wetting: a molecular dynamics study, Journal of Physics; Condensed Matter 21, 464124. 
Blake, T.D. \& Hayness, J.M. (1969), Kinetics of liquid/liquid displacement, Journal of Colloid and Interface Science, 30, p.421-423.

Blake, T.D., Clarke, A., Ruschak, K.J. (1994), Hydrodynamic Assist of Dynamic Wetting, AIChE Journal, 40(2), p.229-242.

Blake, T.D., Clarke, A. (1997), Contact Angle Relaxation during Droplet Spreading: Comparison between Molecular Kinetic Theory and Molecular Dynamics, Langmuir, $13,2164-2166$.

Blake, T.D., \& De Coninck, J. (2002), The influence of solid-liquid interactions on dynamic wetting, Advances in Colloid and Interface Science, 96, p.21-36.

Blake T.D (2006), The Physics of Moving Wetting Lines, Journal of Colloid and Interface Science, 299(1), p.1-496.

Bonn, D., Eggers, J., Indekheu, J., Meunier, J. \& Rolley, E. (2009), Wetting and Spreading, Reviews of Modern Physics, 81,p.739-805.

Brenner, M.P., \& Bertozzi, A.L. (1993), Spreading of droplets on a solid surface, Physics Review Letters, 71(4), p. 593-596.

Brochard-Wyart, F. \& de Gennes, P.G. (1992), Dynamics of partial wetting, Advance in Colloid Interface Science, 39, p.1-11.

Burkholz, A. (1989), Droplet Separation, VCH Publications, p.173-176.

Burley, R., \& Kennedy, B. S. (1976), An Experimental Study of Air Entrainment at a Solid-Liquid-Gas Interface, Chemical Engineering Science, 31,p.901.

Butt, H.J., Graf, K., \& Kappl, M. (2003), Physics and Chemistry of Interfaces, WileyVCH Verlag GmbH \& Co. KGaA., p.1-176.

Chau, T.T. (2009), A review of techniques for measurement of contact angles and their applicability on mineral surfaces, Minerals Engineering 22, p.213-219.

Chen, J.D., and Wada, N. (1988), Wetting dynamics near the edge of a spreading drop, Physics Review Letters, 62, p.3050. 
Christenson, H.K., Claesson, P.M., Berg, J. \& Herder, P.C. (1989), Forces between fluorocarbon surfactant monolayers: Salt effects on the hydrophobic interactions, Journal of Physical Chemistry, 93, p.1472.

Churaev, N.V. 2005, Aqueous wetting films in contact with a solid phase, Advances in Colloid and Interface Science, 3, p.114-115.

Claesson, P.M., Blom, C.E., Herder, P.C. \& Ninham, B.W. (1986), Interactions between water stable hydrophobic Langmuir-Blodgett monolayers on mica, Journal of Colloid Interface Science, 114, p.234-242.

Cross, J. (1987), Electrostatics in gas filtration, Electrostatics: Principles, Problems and Applications, Adam Hilger, p.144-237.

Cruz, A.B. (1997), A review of the literature on Column Flotation Modelling, $A$ comprehensive Dynamic Model of the Column Flotation Unit Operation (PhD dissertation) Virginia Polytechnic Institute and State University, VA, USA.

Dai, Z., Fornasiero, D., \& Ralston, J. (2000), Particle-bubble collision models - a review. Advances in Colloid and Interface Science, 85(2-3), p. 231-256. DOI: http://dx.doi.org/10.1016/S0001-8686(99)00030-5.

De Coninck, J., de Ruijter, M.J. \& Voue, M. (2000), Dynamics of wetting, Current Opinion, Journal of Colloid and Interface Science, 6, p.49-53.

de Gennes, P.G. (1985), Wetting: statics and dynamics, Reviews of Modern Physics, 57(3), p.827-863.

de Gennes, P.G. (1987), Polymers at an interface; a simplified view, Advances in Colloid and Interface Science, 27, p. 189-209.

Derjaguin, B. V., Churaev, N. V., \& Muller, V. M. (1987), Surface Forces, Plenum Publishing, New York, p. 198-202.

Derjaguin, B.V., \& Dukhin, S.S. (1961), Theory of flotation of small and medium size particles, Transactions of Institute of Mining \& Metallurgy, 70, p.221-246. 
Dobby, G. S., \& Finch, J. A. (1986), A model of particle sliding time for flotation size bubbles, Journal of Colloid and Interface Science, 109(2), p.493-498.

Environmental Protection Agency (EPA) (2010), Control of Particulate Matter Emissions, Characteristics of Particles-Collection Mechanisms, National Ambient Air Quality Standards (NAAQS), 5, p.1-22. http://earth1.epa.gov/air/criteria.html, 2010-0830.

Erbil H.Y. (2006), Molecular Interactions, Surface Chemistry of Solid and Liquid Interfaces, Blackwell, p. 39-47.

Eriksson J.C., Ljunggren S. \& Claesson P.M. (1989), A phenomenological theory of long-range hydrophobic attraction forces based on a square-gradient variational approach, Journal of Chemical Society Faraday Transactions II, 85, p.163-76.

Flagan, R.C. \& Seinfeld, J.H. (1988), Aerosols, Fundamentals of Air Pollution Engineering. Prentice-Hall, Inc., p. 290-356.

Flynn, D. (2009), Wet Gas Scrubbers, Nalco Water Hand Book, $3^{\text {rd }}$ ed., McGraw Hill Professional, p.28.

Fuerstenau, M.C., Jameson, G. \& Yoon, R.H. (2007), Flotation Fundamentals, Froth Flotation-A Century of Innovation, Society for Mining, Metallurgy and Exploration, Inc, (SME), p.93- 132.

Galvin, K.P., Walton, K. \& Zhou. J. (2010), Application of Closely Spaced Inclined Channels in Gravity Separation of Fine Particles, Minerals Engineering, 23, p.326-338.

Galvin, K.P., Webber G.B., Mason, M. \& Liyanaarachchi K.R. (2010), Inverse Flotation-A new method of fine article beneficiation, Proceedings $40^{\text {th }}$ Annual Australian Chemical Engineering Conference, CHEMECA 2010, Adelaide, Australia.

Gamota, D., Brazis, P., Kalyanasundaram, K., Zhang, J. (2004), Printed organic and molecular electronics, Kluwer Academic Publications, Massachusetts, USA.

Gaudin, A.M. (1957), Flotation, $2^{\text {nd }}$ ed., McGraw Hill Publications, p.157-162. 
Greenspan, H. P. (1978), On the motion of a small viscous droplet that wets the surface, Journal of Fluid Mechanics, 84, p. 125-143.

Good, R.J. (1992), Contact angle, wetting, and adhesion; a critical review, Journal of Adhesion Science Technology, 6(12), p.1269-1302.

Gupta A. \& Yan D.S. (2006), Flotation, Mineral Processing Design and Operation: An Introduction, Elsevier Science, p. 555-603.

Gupta, R., Gidaspow, D. \& Wasan, D.T. (1993), Electrostatic separation of powder mixtures based on the work functions of its constituents, Powder Technology, 75, p.7987.

Haider, A., \& Levenspiel, O. (1989), Drag coefficient and terminal settling velocity of spherical and non-spherical particles, Powder Technology, 77, p.143-152.

Harkins, W. D. (1952), The Physical Chemistry of Surface Films. Reinhold, New York. DOI: 10.1016/0016-0032(52)90624-8.

Hocking, L.M. (1992), Rival contact angle models and the spreading of drops, Journal of Fluid Mechanics, 239, p. 671-681.

Hocking, L.M. (1994), The spreading of drops with intermolecular forces, Physics of Fluids, 6(10), p.3224-3229.

Ireland, P.M. (2012), Dynamic particle-surface tribocharging: The role of shape and contact mode, Journal of Electrostatics, 70(6), p.524-531.

Israelachvili, J. N. (2011), Special Interactions: Hydrogen-Bonding and Hydrophobic and Hydrophilic Interactions, Intermolecular and Surface Forces, 3rd ed., Elsevier Publishes, p.133-158.

Israelachvili, J.N. (1992), Solvation, structural and hydration forces, Intermolecular and surface forces, $2^{\text {nd }}$ ed., Academic Press, p.260-282.

Israelachvili, J., \& Pashley, R. (1982), The hydrophobic interaction is long range, decaying exponentially with distance. Nature, 300(5890), p.341-342. DOI: $10.1038 / 300341 \mathrm{a} 0$. 
Jameson, G.J., Nam, S., \& Moo-Young, M. (1977), Physical factors affecting recovery rates in flotation, Minerals Science and Engineering, 9(3), p. 103-118.

Janczuk, B. (2006), Adhesion of air bubbles to mineral surfaces in the presence of liquids, Encyclopedia of Surface and Colloid Science, 1(1), p.97-112.

Jowett, A., Somasundaran P. ed. (1980), Formation and Disruption of Particle-Bubble Aggregates in Flotation, Fine Particles Processing, 1, AIME, p.720-754.

Kelly, E.G., \& Spottiswood, D.J. (1989), The theory of electrostatic separations: A review part I. Fundamentals, Minerals Engineering, 2(1), p. 33-46.

King, R.P. (2002), Interaction between fluids and particles, Introduction to particle fluid flow, Butterworth Heinemann, p.59-65.

Lacks, D.J. 2012, The unpredictability of electrostatic charging, Angewandte Chemie International Edition, 51(28), p.6822-6823.

Langmuir I., \& Blodgett, K. (1945), Mathematical investigation of water droplet trajectories, General Electric Company Publication., 129.

Laskowski, J. \& Kitchener, J.A.(1969), Hydrophilic-hydrophobic transition on silica, Journal of Colloid \& Interface Science, 30, p.391.

Lechner, M.D. \& Wohlfarth, Ch. (2008), Static dielectric constants of pure liquids and binary liquid mixtures, Landolt-Bornstein - Group IV Physical Chemistry, 17(IV/6), p. 360-362.

Liang, Y., Hilal, N., Langstong, P. \& Starov, V. (2007), Interaction forces between colloidal particles in liquid: Theory and Experiment, Advances in Colloid and Interface Science, 134-135, p. 151-166.

Liu, Y., German, R.M. (1996), Contact angle and Solid-Liquid-Vapor Equilibrium, Acta Materials, 44(4), p.1657-1663.

Liyanaarachchi, K.R., Ireland, P.M., Webber, G.B., \& Galvin, K.P. (2013), Electrostatic formation of liquid marbles and agglomerates, Applied Physics Letters, 103(5), p.054104- 054105. 
Liyanaarachchi, K.R., Webber, G.B., Van-Netten, K., Moreno-Atanasio, R. \& Galvin, K.P. (2014), Selective Collection of Fine Particles by Water Drops, Advanced Powder Technology, 25(4), p. 1311-1318.

Liu, D., He , Q. \& Evans, G.M. (2010).Flotation separation of Mixtures of Coal and Silica Particles onto a Moving Water Surface, Proceedings, $40^{\text {th }}$ Annual Australian Chemical Engineering Conference, CHEMECA 2010, Adelaide, Australia.

Lowell, J. and Rose-Innes, A.C. (1980), Contact electrification, Advances in Physics, 29 (6), p.947-1023.

Luttrell, G.H., \& Yoon, R. H. (1992), A Hydrodynamic Model for Bubble-Particle Attachment, Journal of Colloid and Interface Science, 154(1), p.129-137.

DOI: http://dx.doi.org/10.1016/0021-9797(92)90085-Z

Mahadevan, L. \& Pomeau, Y. (1999), Rolling droplets. Physics of Fluids, 11(9), p. 2449-2453.

Manouchehri, H. R.; Rao, K. Hanumantha \& Forssberg, K. S. E. (2002), Triboelectric Charge, Electrophysical Properties and Electrical Beneficiation Potential of Chemically Treated Feldspar, Quartz and Wollastonite. Magnetic \& Electrical Separation, 11(1/2), p.1-24.

Mao, L. (1998), Application of extended DLVO theory; Modelling of Flotation and Hydrophobicity of Dodecane (PhD Dissertation), Virginia Polytechnic Institute and State University, p.14-17.

Marchand, K. A., (2012), Utilization of Biodiesel Derived Crude Glycerol by Fungi for Biomass and Lipid Production (PhD Dissertation), University of Guelph, Ontario, Canada.

Masuda, H., Gotoh, K., Higashitani, K. \& Matsusaka, S. (2007), Adhesive force of a single particle, Powder Technology: Fundamentals of Particles, Powder Beds and Particle Generation, Taylor and Francis, p.157-170. 
Masuda, H., Higashitani, K. \& Yoshida, H. (2007), Powder Technology: Handling and Operations, Process Instrumentation, and Working Hazards, Taylor and Francis, p.205215.

Matsusaka, S. (2011), Control of particle tribocharging, KONA Powder and Particle Journal, 29, p.27-38.

McHale, G., Newton, M.I., \& Shirtcliffe, N.J. (2009), Dynamic wetting and spreading and the role of topography, Journal of Physics; Condensed Matter, 21, p.464122.

McHale, G., \& Newton, M.I. (2011), Liquid Marbles: Principles and Applications, Soft Matter, 7 (12), p. 5473-5481.

Miettinen, T., Ralston, J. \& Fornasiero, D. (2010), The limits of fine particle flotation, Minerals Engineering, 23, p.420-437.

Mika, T.S. \& Fuerstenau, D.W. (1968), A microscopic model of the flotation process, Proc., $8^{\text {th }}$ International Mineral Processing Congress, 2, paper $S-4$.

Moore, A.D. (1973), Introduction, Electrostatics and its applications, WileyInterscience Publication, p.1-25.

Mulligan, J.C. (2003), Handling flammable liquids, Liquids Handling, CEP Magazine, July 2003, p.48-56.

Nguyen, A.V. (1994), The Collision between Fine Particles and Single Air Bubbles in Flotation, Journal of Colloid and Interface Science, 162(1), p.123-128.

Nguyen, A. V., \& Evans, G. M. (2004), Attachment interaction between air bubbles and particles in froth flotation. Experimental Thermal and Fluid Science, 28(5), p.381-385. DOI: http://dx.doi.org/10.1016/j.expthermflusci.2002.12.001

Nguyen, A.V. \& Kmet, S. (1992), Collision efficiency for fine mineral particles with single bubble in a countercurrent flow regime. International Journal of Mineral Processing, 35(3-4), p.205-223.

Nguyen, A.V., Schulze, H.J. (2004), Colloid Science of Flotation, Surfactant Science Series, 118, Marcel Dekker, p.295-801. 
Nguyen, A.V., Schulze, H.J. \& Ralston, J. (1997), Elementary steps in particle-bubble attachment, International Journal of Mineral Processing, 51 (1-4), p. 183-195.

Nicol, S.K. (2001), Fine coal beneficiation. In: Swanson, A.R., Partridge, A.C. (Eds.), Advanced Coal Preparation Monograph Series, Vol. IV, Part 9, p.107-136.

Paulsen, F.G., Pan, R., Bousfield, D.W. \& Thompson, E.V. (1996), The Dynamics of Bubble / Particle Attachment and the Application of Two Disjoining Film Rupture Models to Flotation I. Nondraining Model, Journal of Colloid and Interface Science, 178, p. $400-410$.

Peckham, D.E. (1996), Work of adhesion: contact angles and contact mechanics, International Journal of Adhesion and Adhesives, 16(2), p.121-128.

Pethig, R. (2010), Review Article-Dielectrophoresis: Status of the theory, technology, and applications, Biomicrofluidics, 4(2), p.022811.

Petrov, P.G., \& Petrov. J.G. (1992), A combined molecular-hydrodynamic approach to wetting kinetics, Langmuir, 8, p.1762-1767.

Phan, C.M., Nguyen A.V., Miller, J.D., Evans, G.M. \& Jameson G.J. (2003), Investigations of bubble-particle interactions, International Journal of Mineral Processing, 72, p.239- 254.

Pohl, H.A. (1958) Some Effects of nonuniform fields on dielectrics. Journal of Applied Physics, 29, p.1182-1188.

Pohl, H.A., Plymale, C.E. (1960) Continuous separations of suspensions by nonuniform electric fields in liquid dielectrics, Journal of the Electrochemical Society 107, p.390396.

Ralston, J. \& Dukhin, S.S. (1999), The interaction between particles and bubbles, Colloids and Surfaces, A: Physiochemical and Engineering Aspects 151,15, p.3-14.

Ralston, J., Fornasiero, D., \& Hayes, R. (1999), Bubble-particle attachment and detachment in flotation. International Journal of Mineral Processing, 56(1-4), p.133164. DOI: http://dx.doi.org/10.1016/S0301-7516(98)00046-5 
Ralston, J., Popescu, M., \& Sedev, R. (2008), Dynamics of Wetting from an

Experimental Point of View, Annual Review of Materials Research, 38(1), p. 23-43.

DOI: 10.1146/annurev.matsci.38.060407.130231

Rao, S.R. \& Leja, J. (2004), Surface chemistry of froth flotation, $2^{\text {nd }}$ ed., Vol.2, p. 257292.

Roura, P. \& Fort, J. (2004), Local thermodynamic derivation of Young's equation, Journal of Colloid and Interface Science, 272, p.420-429.

Ruckenstein E. \& Churaev N. (1991), A Possible Hydrodynamic Origin of the Forces of Hydrophobic Attraction, Journal of Colloid and Interface Science, 147, p.535-8.

Schnelle, K.B. \& Brown, C.A. (2002), Design and Application of Wet Scrubbers, Air Pollution Control Technology Handbook, CRC press, p.1-29.

Schrader, M.E. (1995), Young -Dupre Revisited, Langmuir, 11, p.3585- 3589.

Schulze, H. J. (1984), Physico-chemical elementary processes in flotation: an analysis from the point of view of colloid science including process engineering considerations, Elsevier Publications .

Schulze, H. J. (1992), Probability of particle attachment on gas bubbles by sliding. Advances in Colloid and Interface Science, 40, p.283-305.

Seville, J.P.K, Tuzun, U. \& Clift, R. (1997), Particles in Fluids, Processing of Particulate Solids, Blackie Academic \& Professional Publications, p.53-285.

Shahbazi, B., Rezai, B. \& Koleini S.M.J. (2010), Bubble-particle collision and attachment probability of fine particles flotation, Chemical Engineering and Processing, 49, p.622-627.

Sharma, P.K. \& Rao, H.K. (2002), Analysis of different approaches for evaluation of surface energy of microbial cells by contact angle goniometry, Advances in Colloid and Interface Science, 98, p.341- 463.

Shikhmurzaev, Y.D. (1994), Moving Contact Lines in Liquid/Liquid/Solid Systems, Journal of Fluid Mechanics, 334, p. 211-249. 
Shang, J., Flury, M .\& Deng, Y. (2009), Force measurements between particles and the air-water interface: Implications for particle mobilization in unsaturated porous media, Water Resources Research, 45. (6), W06420. DOI: 10.1029/2008wr007384

Somasundaran, P., Mehta, S.C., Yu, X. \& Krishnakumar, S. (2009), Colloid systems and interfaces and interfaces stability of dispersions through Polymer and Surfactant adsorption, Introduction to Particle Technology, Taylor and Francis, p.155-194.

Starov,V.M., Velarde, M.G. \& Radke, C.J. (2007), Wetting and Spreading, Surfactant Science Series, 138 , p.515.

Sutherland, K.L. (1948), Physical chemistry of flotation XI, Kinetics of the flotation process, Journal of Physical Chemistry, 52,p. 394-425.

Sutherland, K. L \& Wark, I.W. (1955), Principles of flotation (Revised and enlarged ed.). Australasian Institute of Mining and Metallurgy, Melbourne.

Szatkowski, M. (1987), Factors influencing behaviour of flotation froth, Transactions of Institute of Mining \& Metallurgy, Sect. C 96, p.115-122.

Tanner, L.H. (1979), The spreading of silicone oil drops on horizontal surfaces, Journal of Physics D: Applied Physics, 12, p.1473.

Tian, J., Arbatan, T., Li,X., \& Shen, W. (2010), Liquid marble for gas sensing, Chemical Communications, 46, p.4734-4736.

Tien, C. \& Ramarao, B.V. (2007), Introduction, Granular Filtration of Aerosols and Hydrosols, $2^{\text {nd }}$ ed., Elsevier, p.1-7.

Tran, D. N. H., Whitby, C. P., Fornasiero, D. \& Ralston, J. (2010), Selective separation of very fine particles at a planar air-water interface, International Journal of Mineral Processing, 94,1-2, p.35-42.

Tsai, S.C. (1985), Effects of Surface Chemistry and Particle Size and Density on Froth Flotation of Fine Coal, Colloids and Surfaces, 16, p.323-336. 
Tsao, Y. H., Yang, S. X., Evans, D. F., \& Wennerstroem, H. (1991), Interactions between hydrophobic surfaces- Dependence on temperature and alkyl chain length. Langmuir, 7(12), p.3154-3159. DOI: 10.1021/la00060a039

Verwey, E. J. W. and Overbeek, J. Th. G. 1948, Theory of the Stability of Lyophobic Colloids, Elsevier, New York, pp. 106-115;

Voinov, O. V. (1976), Hydrodynamics of wetting, Journal of Fluid Dynamics, 11(5), p.714-721. DOI: $10.1007 /$ bf01012963

Wåhlin, A., Bäckström, G. (1974), Sliding electrification of Teflon by metals, Journal of Applied Physics, 45, p.2058-2064.

Weber, M.E. \& Paddock, D. (1983), Interceptional and gravitational collision efficiencies for single collectors at intermediate Reynolds numbers, Journal of Colloid and Interface Science, 94 (2), p. 328-335.

Wilkinson, M. C., \& Elliott, T. A. (1974), Dynamic contact angles in mercury/carbon tetrachloride/solution systems. II. The process of attachment. Journal of Colloid and Interface Science, 48(2), p. 209-224. DOI: http://dx.doi.org/10.1016/00219797(74)90156-8.

Wyart, B., \& de Gennes, P.G. (1992), Dynamics of partial wetting, Advances in Colloid and Interface Science, 39, p.1-11.

Xu. Z., Huang, X. \& Wan, L. (2009), Wettability of Membrane Surfaces, Surface Engineering of Polymer Membranes, Zhegiang University Press \& Springer GmBH, p.44-50.

Xu. Z. \& Yoon, R.H. (1989), The Role of Hydrophobic Interactions in Coagulation, Journal of Colloid and Interface Science, 132(2), p. 532-541.

Ye, Y., \& Miller, J.D. (1988), Bubble-particle contact time in the analysis of coal flotation. Coal Preparation 5 (3-4), p.147-166.

Yarar, B. (2000), Flotation, Kirk-Othmer Encyclopedia of Chemical Technology, John Wiley and Sons, Inc., DOI: 10.1002/14356007.b02_23 
Yoon, R. H. (2000), The role of hydrodynamic and surface forces in bubble-particle interaction. International Journal of Mineral Processing, 58(1-4), p.129-143.

DOI: http://dx.doi.org/10.1016/S0301-7516(99)00071-X

Yoon, R. H. \& Luttrell, G. H. (1986), The Effect of Bubble Size on Fine Coal Flotation, Coal Preparation, 2(3), p.179-192.

Yoon, R. H., \& Luttrell, G. H. (1989), The Effect of Bubble Size on Fine Particle Flotation. Mineral Processing and Extractive Metallurgy Review, 5(1-4), 101-122.DOI: $10.1080 / 08827508908952646$

Yoon, R. H., \& Mao, L. (1996), Application of extended DLVO theory, IV: Derivation of flotation rate equation from first principles. Journal of Colloid and Interface Science, 181(2), p.613-626.

Young, H.D. (1991), University Physics, $8^{\text {th }}$ ed., Addison-Wesley Publishing Company, United States. p. 605-707.

Young. T. (1805), An essay on the cohesion of fluid. Philosophical Transactions, Read December, 20, p. 65.

Zettlemoyer, A.C. (1968), Hydrophobic Surfaces, Journal of Colloid and Interface Science, 28(3-4), p. 343-369.

Zisman, W. A. (1964), Relation of the equilibrium contact angle to liquid and solid constitution, Advances in Chemistry Series, 43, p.1-49.

Zhou, Z.A., Xu, Z. \& Finch, J.A., (1994), On the role of cavitation in particle collection during flotation - a critical review, Minerals Engineering, 7 (9), p.1073-1084.

Zhou, Z.A., Xu, Z., Finch, J.A., Hu, H. \& Rao, S.R.(1997), Role of hydrodynamic cavitation in fine particle flotation. International Journal of Mineral Processing, 51 (14), p.139-149.

Zongfu, D., Fornasiero, D. \& Ralston, J. (2000), Particle- Bubble Collision Models-A Review, Advances in Colloid and Interface Science, 85, p.231-256. 


\title{
APPENDICES
}

\author{
APPENDIX A
}

\section{Particle Size Distributions}

Figure A.1: Particle size distribution of 23-47 $\mu \mathrm{m}$ ballotini as received and cleaned glass ballotini fraction as measured by the Malvern Mastersizer. $d_{10}=23 \mu \mathrm{m} ; d_{50}=$ $33 \mu \mathrm{m} ; d_{90}=47 \mu \mathrm{m}$.

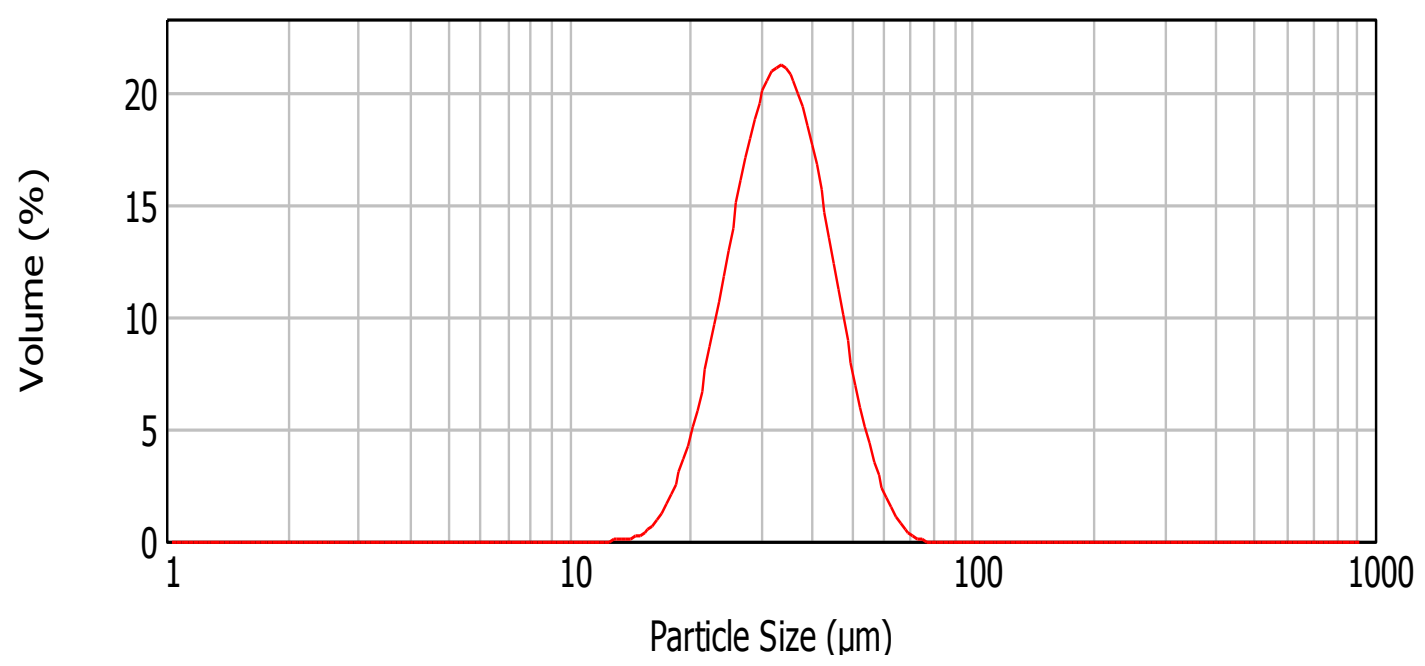

Table A.1: Volume distribution data for 38-45 micron glass ballotini as measured by the Malvern Mastersizer.

\begin{tabular}{|r|r|}
\hline Size $(\mu \mathrm{m})$ & Volume $\ln \%$ \\
\hline 0.010 & 0.00 \\
0.011 & 0.00 \\
0.013 & 0.00 \\
0.015 & 0.00 \\
0.017 & 0.00 \\
0.020 & 0.00 \\
0.023 & 0.00 \\
0.026 & 0.00 \\
0.030 & 0.00 \\
0.035 & 0.00 \\
0.040 & 0.00 \\
0.046 & 0.00 \\
0.052 & 0.00 \\
0.060 & 0.00 \\
0.069 & 0.00 \\
0.079 & 0.00 \\
0.091 & 0.00 \\
0.105 & \\
\hline
\end{tabular}

\begin{tabular}{|r|r|}
\hline Size $(\mu \mathrm{m})$ & Volume $\ln \%$ \\
\hline 0.105 & 0.00 \\
0.120 & 0.00 \\
0.138 & 0.00 \\
0.158 & 0.00 \\
0.182 & 0.00 \\
0.209 & 0.00 \\
0.240 & 0.00 \\
0.275 & 0.00 \\
0.316 & 0.00 \\
0.363 & 0.00 \\
0.417 & 0.00 \\
0.479 & 0.00 \\
0.550 & 0.00 \\
0.631 & 0.00 \\
0.724 & 0.00 \\
0.832 & 0.00 \\
0.955 & 0.00 \\
1.096 & \\
\hline
\end{tabular}

\begin{tabular}{|r|r|}
\hline Size $(\mu \mathrm{m})$ & Volume $\ln \%$ \\
\hline 1.096 & 0.00 \\
1.259 & 0.00 \\
1.445 & 0.00 \\
1.660 & 0.00 \\
1.905 & 0.00 \\
2.188 & 0.00 \\
2.512 & 0.00 \\
2.884 & 0.00 \\
3.311 & 0.00 \\
3.802 & 0.00 \\
4.365 & 0.00 \\
5.012 & 0.00 \\
5.754 & 0.00 \\
6.607 & 0.00 \\
7.586 & 0.00 \\
8.710 & 0.00 \\
10.000 & 0.00 \\
11.482 & \\
\hline
\end{tabular}

\begin{tabular}{|r|r|}
\hline Size $(\mu \mathrm{m})$ & Volume $\ln \%$ \\
\hline 11.482 & 0.00 \\
13.183 & 0.14 \\
15.136 & 1.00 \\
17.378 & 3.38 \\
19.953 & 7.62 \\
22.909 & 13.07 \\
26.303 & 17.70 \\
30.200 & 19.32 \\
34.674 & 16.96 \\
39.811 & 11.77 \\
45.709 & 6.22 \\
52.481 & 2.31 \\
60.256 & 0.50 \\
69.183 & 0.01 \\
79.433 & 0.00 \\
91.201 & 0.00 \\
104.713 & 0.00 \\
120.226 & \\
\hline
\end{tabular}

\begin{tabular}{|r|r|}
\hline Size $(\mu \mathrm{m})$ & Volume $\ln \%$ \\
\hline 120.226 & 0.00 \\
138.038 & 0.00 \\
158.489 & 0.00 \\
181.970 & 0.00 \\
208.930 & 0.00 \\
239.883 & 0.00 \\
275.423 & 0.00 \\
316.228 & 0.00 \\
363.078 & 0.00 \\
416.869 & 0.00 \\
478.630 & 0.00 \\
549.541 & 0.00 \\
630.957 & 0.00 \\
724.436 & 0.00 \\
831.764 & 0.00 \\
954.993 & 0.00 \\
1096.478 & 0.00 \\
1258.925 & \\
\hline
\end{tabular}

\begin{tabular}{|r|r|}
\hline Size $(\mu \mathrm{m})$ & Volume In $\%$ \\
\hline 1258.925 & 0.00 \\
1445.440 & 0.00 \\
1659.587 & 0.00 \\
1905.461 & 0.00 \\
2187.762 & 0.00 \\
2511.886 & 0.00 \\
2884.032 & 0.00 \\
3311.311 & 0.00 \\
3801.894 & 0.00 \\
4365.158 & 0.00 \\
5011.872 & 0.00 \\
5754.399 & 0.00 \\
6606.934 & 0.00 \\
7585.776 & 0.00 \\
8709.636 & 0.00 \\
10000.000 & \\
& \\
& \\
\hline
\end{tabular}


Figure A.2: Particle size distribution of 17-36 $\mu \mathrm{m}$ Cleaned glass ballotini fraction as measured by the Malvern Mastersizer. $d_{10}=17 \mu \mathrm{m} ; d_{50}=25 \mu \mathrm{m} ; d_{90}=36 \mu \mathrm{m}$.

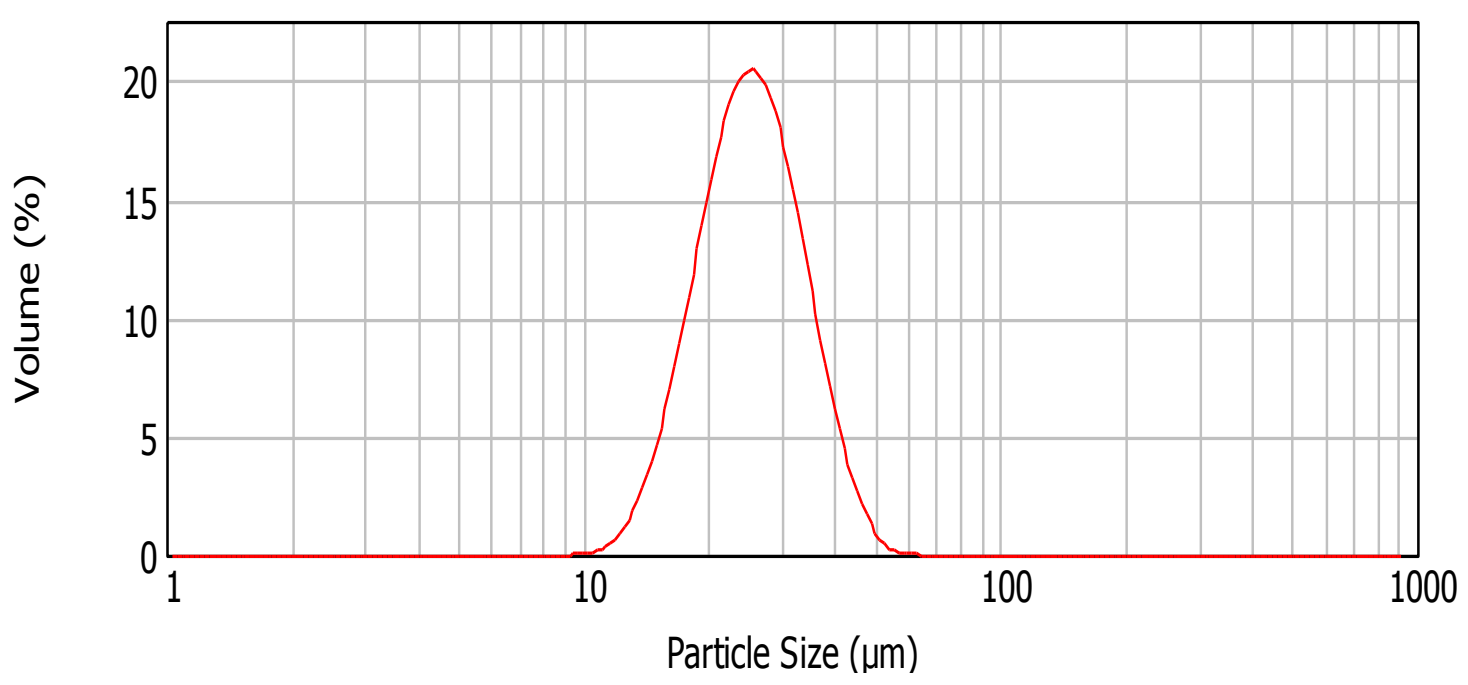

Table A.2: Volume frequency distribution data for $\mathbf{- 3 8}$ micron glass ballotini as measured by the Malvern Mastersizer.

\begin{tabular}{|c|c|c|c|c|c|c|c|c|c|c|c|}
\hline Size (mm) & Volume (\%) & Size $\left(\mu_{m}\right)$ & Volume (\%) & Size $\left(\mu_{m}\right)$ & Volume (\%) & Size $\left(\mu_{m}\right)$ & Volume (\%) & Size $\left(\mu_{\mathrm{m}}\right)$ & Volume (\%) & Size $\left(\mu_{\mathrm{m}}\right)$ & Volume (\%) \\
\hline 0.010 & & 0.105 & & 1.096 & & 11.482 & & 120.226 & & 1258.925 & \\
\hline 0.011 & 0.00 & 0.120 & 0.00 & 1.259 & 0.00 & 13.183 & 0.84 & 138.038 & 0.00 & 1445.440 & 0.00 \\
\hline 0.013 & 0.00 & 0.138 & 0.00 & 1.445 & 0.00 & 15.136 & 2.85 & 158.489 & 0.00 & 1659.587 & 0.00 \\
\hline 0.015 & 0.00 & 0.158 & 0.00 & 1.660 & 0.00 & 17.378 & 6.44 & 181.970 & 0.00 & 1905.461 & 0.00 \\
\hline 0.017 & 0.00 & 0.182 & 0.00 & 1.905 & 0.00 & 19.953 & 11.33 & 208.930 & 0.00 & 2187.762 & 0.00 \\
\hline 0.020 & 0.00 & 0.209 & 0.00 & 2.188 & 0.00 & 22.909 & 15.95 & 239.883 & 0.00 & 2511.886 & 0.00 \\
\hline 0.023 & 0.00 & 0.240 & 0.00 & 2.512 & 0.00 & 26.303 & 18.42 & 275.423 & 0.00 & 2884.032 & 0.00 \\
\hline 0.026 & 0.00 & 0.275 & 0.00 & 2.884 & 0.00 & 30.200 & 17.44 & 316.228 & 0.00 & 3311.311 & 0.00 \\
\hline 0.030 & 0.00 & 0.316 & 0.00 & 3.311 & 0.00 & 34.674 & 13.41 & 363.078 & 0.00 & 3801.894 & 0.00 \\
\hline 0.035 & 0.00 & 0.363 & 0.00 & 3.802 & 0.00 & 39.811 & 8.16 & 416.869 & 0.00 & 4365.158 & 0.00 \\
\hline 0.040 & 0.00 & 0.417 & 0.00 & 4.365 & 0.00 & 45.709 & 3.75 & 478.630 & 0.00 & 5011.872 & 0.00 \\
\hline 0.046 & 0.00 & 0.479 & 0.00 & 5.012 & 0.00 & 52.481 & 1.16 & 549.541 & 0.00 & 5754.399 & 0.00 \\
\hline 0.052 & 0.00 & 0.550 & 0.00 & 5.754 & 0.00 & 60.256 & 0.11 & 630.957 & 0.00 & 6606.934 & 0.00 \\
\hline 0.060 & 0.00 & 0.631 & 0.00 & 6.607 & 0.00 & 69.183 & 0.00 & 724.436 & 0.00 & 7585.776 & 0.00 \\
\hline 0.069 & 0.00 & 0.724 & 0.00 & 7.586 & 0.00 & 79.433 & 0.00 & 831.764 & 0.00 & 8709.636 & 0.00 \\
\hline 0.079 & 0.00 & 0.832 & 0.00 & 8.710 & 0.00 & 91.201 & 0.00 & 954.993 & 0.00 & 10000.000 & 0.00 \\
\hline 0.091 & 0.00 & 0.955 & 0.00 & 10.000 & 0.00 & 104.713 & 0.00 & 1096.478 & 0.00 & & \\
\hline 0.105 & 0.00 & 1.096 & 0.00 & 11.482 & 0.15 & 120.226 & 0.00 & 1258.925 & 0.00 & & \\
\hline
\end{tabular}


Figure A.3: Particle size distribution of 38-45 $\mu \mathrm{m}$ cleaned rough silica fraction as measured by the Malvern Mastersizer. $d_{10}=21 \mu \mathrm{m} ; d_{50}=34 \mu \mathrm{m} ; d_{90}=54 \mu \mathrm{m}$.

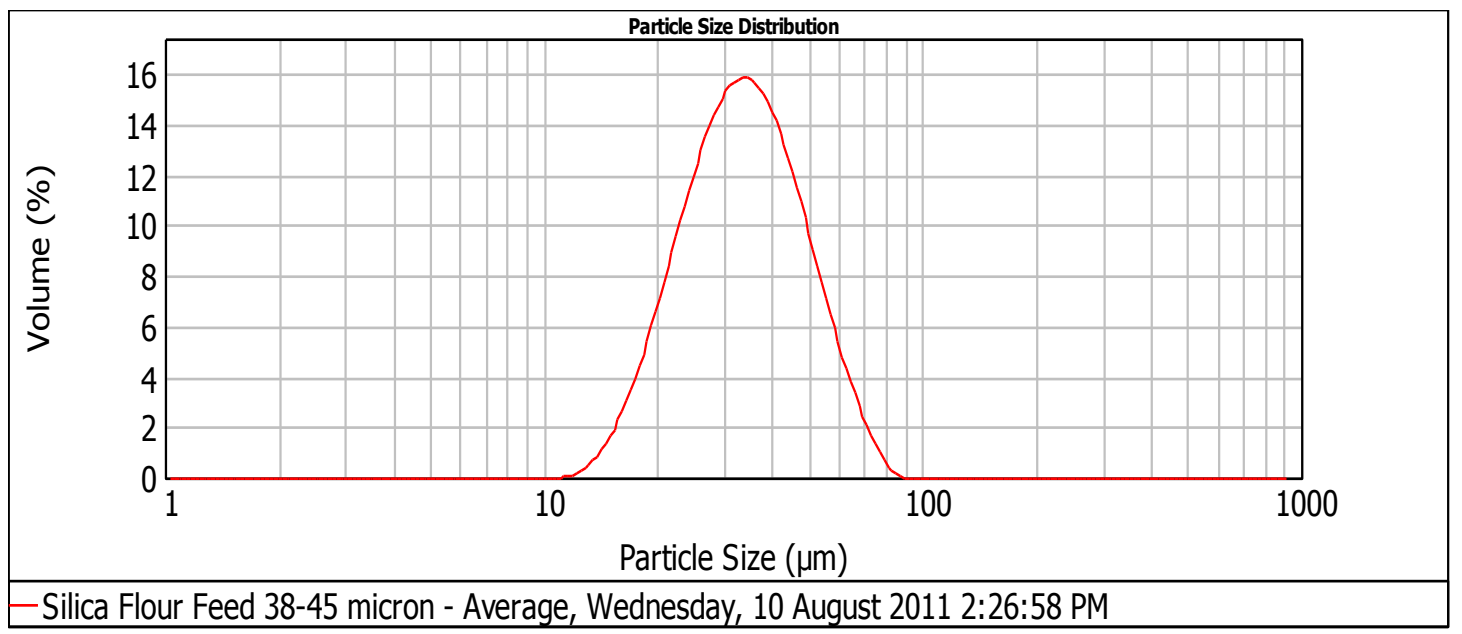

Table A.3: Volume frequency distribution data for 38-45 micron rough silica flour as measured by the Malvern Mastersizer.

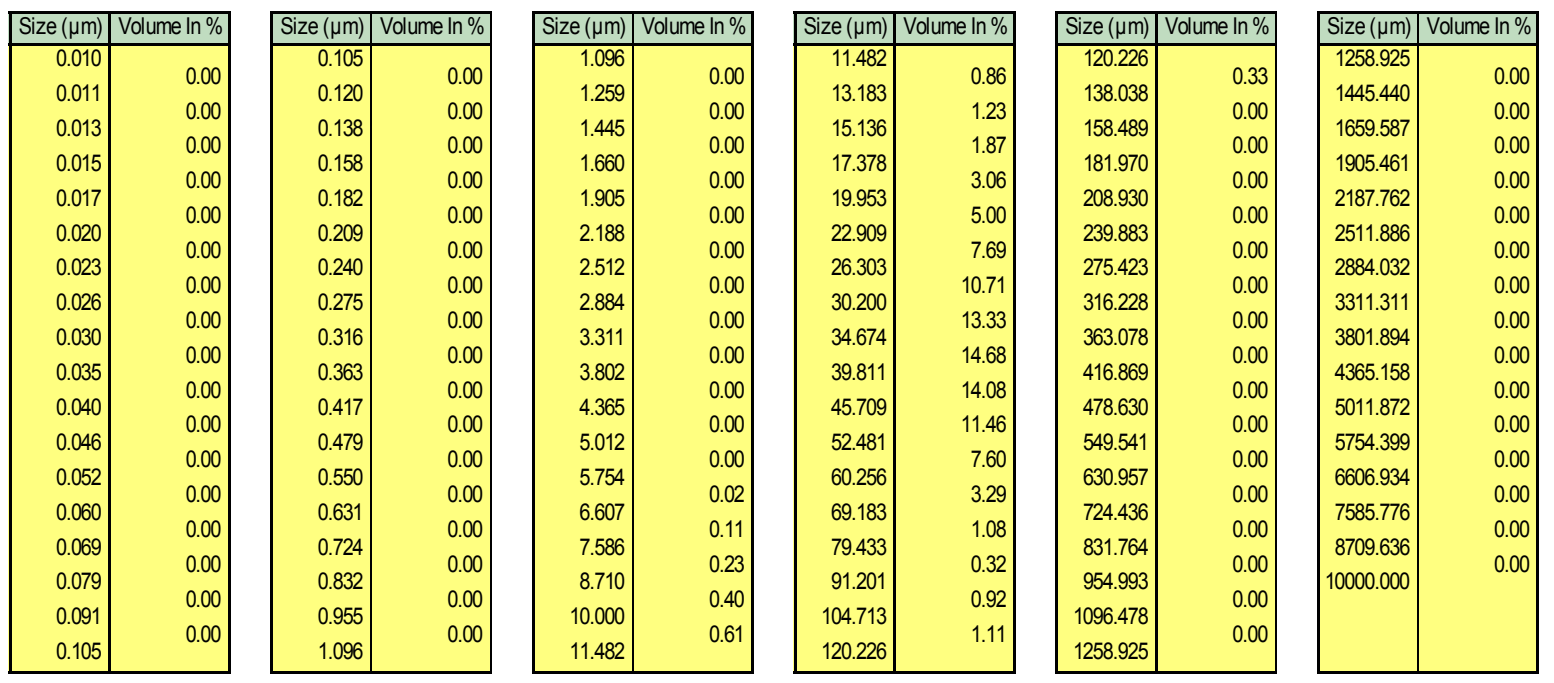


Figure A.4: Particle size distribution of 10-20 $\mu \mathrm{m}$ coal fraction as measured by the Malvern Mastersizer. $d_{10}=8 \mu \mathrm{m} ; d_{50}=16 \mu \mathrm{m} ; d_{90}=29 \mu \mathrm{m}$

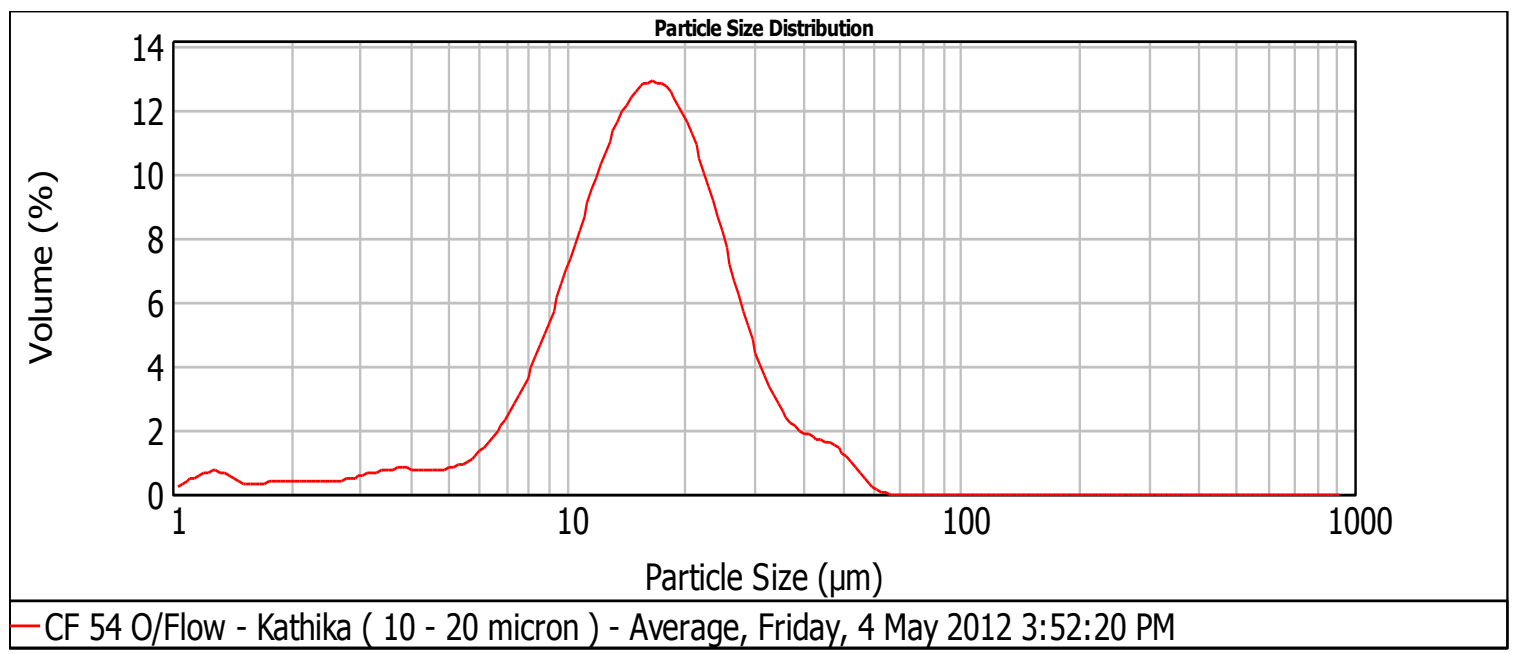

Table A.4: Volume frequency distribution data for 10-20 micron Silica as measured by the Malvern Mastersizer.

\begin{tabular}{|r|r|r|r|}
\hline Size $(\mu \mathrm{m})$ & Volume In \% \\
\hline 0.010 & 0.00 \\
0.011 & 0.00 \\
0.013 & 0.00 \\
0.015 & Size $(\mu \mathrm{m})$ & Volume In \% \\
\hline 0.017 & 0.00 \\
0.020 & 0.00 \\
0.105 & 0.00 \\
0.023 & 0.00 \\
0.026 & 0.138 & 0.00 \\
0.030 & 0.00 \\
0.158 & 0.00 \\
0.035 & 0.00 \\
0.040 & 0.00 \\
0.046 & 0.00 \\
0.052 & 0.00 \\
0.060 & 0.00 \\
0.069 & 0.00 \\
0.079 & 0.240 & 0.00 \\
0.091 & 0.00 \\
0.105 & 0.07 \\
0.316 & 0.00 \\
0.363 & 0.00 \\
0.417 & 0.00 \\
0.479 & 0.00 \\
0.550 & 0.00 \\
0.631 & 0.00 \\
0.724 & 0.00 \\
0.832 & 0.00 \\
0.955 & 0.00 \\
1.096 & 0.00 \\
& & 0.15 \\
\hline
\end{tabular}

\begin{tabular}{|r|r|}
\hline Size $(\mu \mathrm{m})$ & Volume $\ln \%$ \\
\hline 1.096 & 0.52 \\
1.259 & 0.59 \\
1.445 & 0.27 \\
1.660 & 0.33 \\
1.905 & 0.39 \\
2.188 & 0.37 \\
2.512 & 0.39 \\
2.884 & 0.54 \\
3.311 & 0.69 \\
3.802 & 0.70 \\
4.365 & 0.67 \\
5.012 & 0.81 \\
5.754 & 1.29 \\
6.607 & 2.15 \\
7.586 & 3.48 \\
8.710 & 5.25 \\
10.000 & 7.34 \\
11.482 & \\
\hline
\end{tabular}

\begin{tabular}{|r|r|}
\hline Size $(\mu \mathrm{m})$ & Volume $\ln \%$ \\
\hline 11.482 & 9.34 \\
13.183 & 10.91 \\
15.136 & 11.61 \\
17.378 & 11.22 \\
19.953 & 9.77 \\
22.909 & 7.55 \\
26.303 & 5.14 \\
30.200 & 3.14 \\
34.674 & 2.00 \\
39.811 & 1.58 \\
45.709 & 1.28 \\
52.481 & 0.52 \\
60.256 & 0.01 \\
69.183 & 0.00 \\
79.433 & 0.00 \\
91.201 & 0.00 \\
104.713 & 0.00 \\
120.226 & \\
\hline
\end{tabular}

\begin{tabular}{|r|r|}
\hline Size $(\mu \mathrm{m})$ & Volume $\ln \%$ \\
\hline 120.226 & 0.00 \\
138.038 & 0.00 \\
158.489 & 0.00 \\
181.970 & 0.00 \\
208.930 & 0.00 \\
239.883 & 0.00 \\
275.423 & 0.00 \\
316.228 & 0.00 \\
363.078 & 0.00 \\
416.869 & 0.00 \\
478.630 & 0.00 \\
549.541 & 0.00 \\
630.957 & 0.00 \\
724.436 & 0.00 \\
831.764 & 0.00 \\
954.993 & 0.00 \\
1096.478 & 0.00 \\
1258.925 & \\
\hline
\end{tabular}

\begin{tabular}{|r|r|}
\hline Size $(\mu \mathrm{m})$ & Volume $\ln \%$ \\
\hline 1258.925 & 0.00 \\
1445.440 & 0.00 \\
1659.587 & 0.00 \\
1905.461 & 0.00 \\
2187.762 & 0.00 \\
2511.886 & 0.00 \\
2884.032 & 0.00 \\
3311.311 & 0.00 \\
3801.894 & 0.00 \\
4365.158 & 0.00 \\
5011.872 & 0.00 \\
5754.399 & 0.00 \\
6606.934 & 0.00 \\
7585.776 & 0.00 \\
8709.636 & 0.00 \\
10000.000 & \\
& \\
& \\
\hline
\end{tabular}


Figure A.5: Particle size distribution of 20-40 $\mu \mathrm{m}$ Silica fraction as measured by the Malvern Mastersizer. $d_{10}=16 \mu \mathrm{m} ; d_{50}=27 \mu \mathrm{m} ; d_{90}=42 \mu \mathrm{m}$

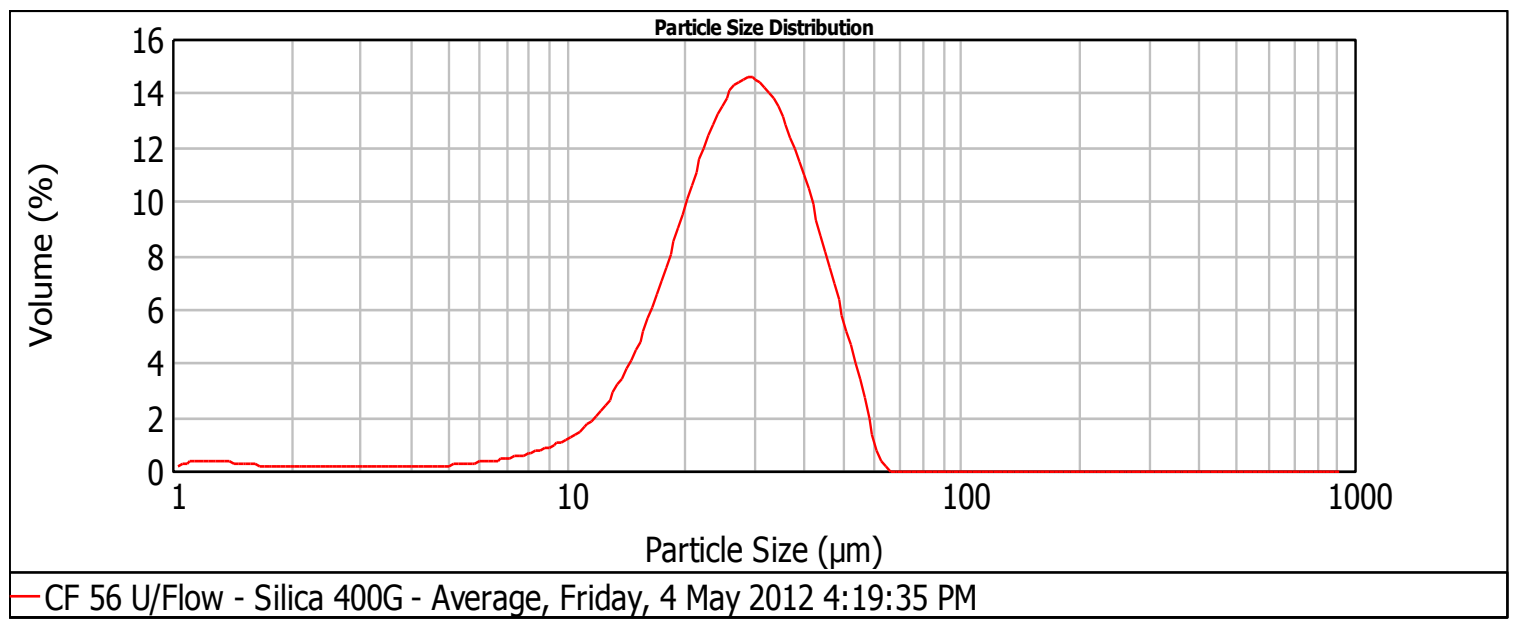

Table A.5: Volume frequency distribution data for 20-40 micron Silica as measured by the Malvern Mastersizer.

\begin{tabular}{|c|c|c|c|}
\hline $\operatorname{Size}(\mu \mathrm{m})$ & Volume $\ln \%$ & Size $(\mu \mathrm{m})$ & Volume $\ln \%$ \\
\hline 0.010 & $\cap \Omega 0$ & 0.105 & 000 \\
\hline 0.011 & 0.00 & 0.120 & 0.00 \\
\hline 0.013 & 0.00 & 0.138 & 0.00 \\
\hline 0.015 & 0.00 & O158 & 0.00 \\
\hline 0.017 & 0.00 & & 0.00 \\
\hline 0.020 & 0.00 & 020 & 0.00 \\
\hline 0.023 & 0.00 & 0240 & 0.00 \\
\hline 0.026 & 0.00 & 0275 & 0.00 \\
\hline 0.030 & 0.00 & 021 & 0.00 \\
\hline 0.035 & 0.00 & 030 & 0.00 \\
\hline 0.040 & 0.00 & 0417 & 0.00 \\
\hline 0.046 & 0.00 & 0470 & 0.00 \\
\hline 0,052 & 0.00 & 0550 & 0.00 \\
\hline 0,060 & 0.00 & 0021 & 0.00 \\
\hline 0069 & 0.00 & 0.001 & 0.00 \\
\hline 0.079 & 0.00 & $0.1<4$ & 0.00 \\
\hline 0.091 & 0.00 & 005 & 0.00 \\
\hline 0.105 & 0.00 & & 0.11 \\
\hline
\end{tabular}

\begin{tabular}{|r|r|}
\hline Size $(\mu \mathrm{m})$ & Volume In \% \\
\hline 1.096 & 0.31 \\
1.259 & 0.31 \\
1.445 & 0.20 \\
1.660 & 0.16 \\
1.905 & 0.13 \\
2.188 & 0.14 \\
2.512 & 0.15 \\
2.884 & 0.14 \\
3.311 & 0.14 \\
3.802 & 0.13 \\
4.365 & 0.14 \\
5.012 & 0.19 \\
5.754 & 0.28 \\
6.607 & 0.41 \\
7.586 & 0.59 \\
8.710 & 0.84 \\
10.000 & 1.28 \\
11.482 & \\
\hline
\end{tabular}

\begin{tabular}{|r|r|}
\hline Size $(\mu \mathrm{m})$ & Volume $\ln \%$ \\
\hline 11.482 & 2.03 \\
13.183 & 3.29 \\
15.136 & 5.12 \\
17.378 & 7.50 \\
19.953 & 10.01 \\
22.909 & 12.09 \\
26.303 & 13.10 \\
30.200 & 12.75 \\
34.674 & 11.16 \\
39.811 & 8.68 \\
45.709 & 5.72 \\
52.481 & 2.77 \\
60.256 & 0.09 \\
69.183 & 0.00 \\
79.433 & 0.00 \\
91.201 & 0.00 \\
104.713 & 0.00 \\
120.226 & \\
\hline
\end{tabular}

\begin{tabular}{|r|r|}
\hline Size $(\mu \mathrm{m})$ & Volume $\ln \%$ \\
\hline 120.226 & 0.00 \\
138.038 & 0.00 \\
158.489 & 0.00 \\
181.970 & 0.00 \\
208.930 & 0.00 \\
239.883 & 0.00 \\
275.423 & 0.00 \\
316.228 & 0.00 \\
363.078 & 0.00 \\
416.869 & 0.00 \\
478.630 & 0.00 \\
549.541 & 0.00 \\
630.957 & 0.00 \\
724.436 & 0.00 \\
831.764 & 0.00 \\
954.993 & 0.00 \\
1096.478 & 0.00 \\
1258.925 & \\
\hline
\end{tabular}

\begin{tabular}{|r|r|}
\hline Size $(\mu \mathrm{m})$ & Volume $\ln \%$ \\
\hline 1258.925 & 0.00 \\
1445.440 & 0.00 \\
1659.587 & 0.00 \\
1905.461 & 0.00 \\
2187.762 & 0.00 \\
2511.886 & 0.00 \\
2884.032 & 0.00 \\
3311.311 & 0.00 \\
3801.894 & 0.00 \\
4365.158 & 0.00 \\
5011.872 & 0.00 \\
5754.399 & 0.00 \\
6606.934 & 0.00 \\
7585.776 & 0.00 \\
8709.636 & 0.00 \\
10000.000 & \\
& \\
& \\
\hline
\end{tabular}


Figure A.6: Particle size distribution of 38-90 $\mu \mathrm{m}$ coal fraction as measured by the Malvern Mastersizer. $d_{10}=29 \mu \mathrm{m} ; d_{50}=64 \mu \mathrm{m} ; d_{90}=105 \mu \mathrm{m}$

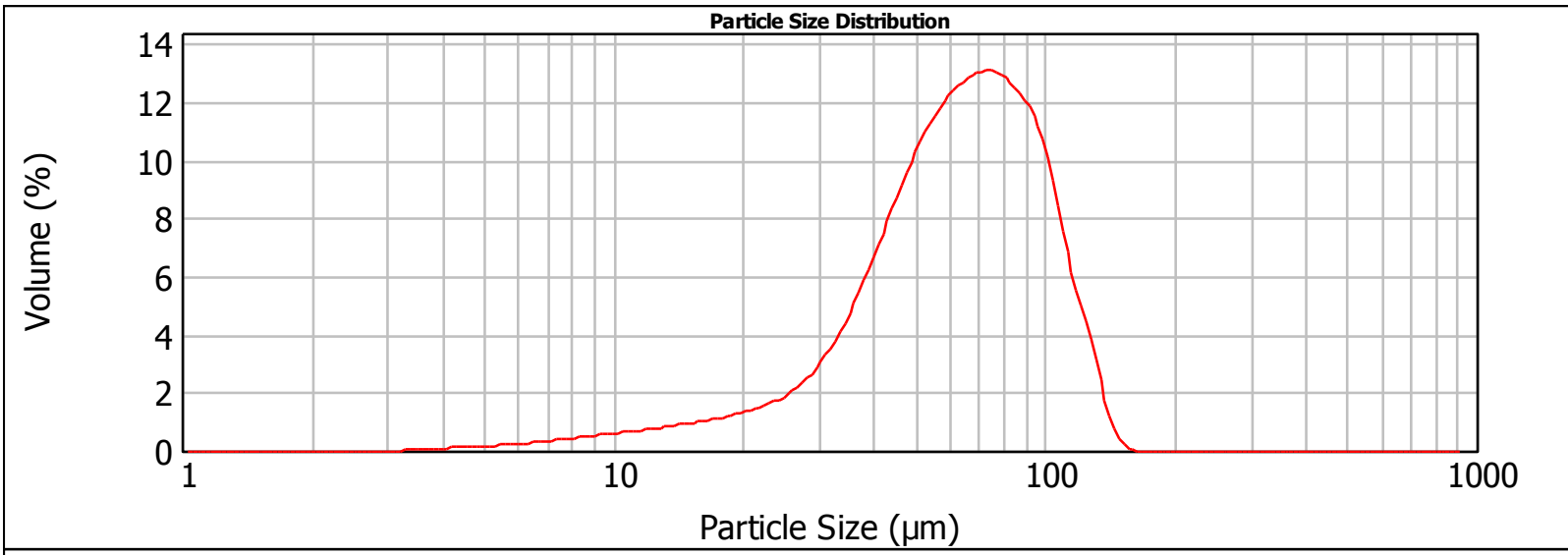

Coal 0_90 25_11_11 - Average, Friday, 25 November 2011 4:36:16 PM

Table A.6: Volume frequency distribution data for 38-90 micron coal as measured by the Malvern Mastersizer.

\begin{tabular}{|r|r|}
\hline Size $(\mu \mathrm{m})$ & Volume $\ln \%$ \\
\hline 0.010 & 0.00 \\
0.011 & 0.00 \\
\hline 0.013 & 0.00 \\
0.015 & 0.00 \\
0.017 & 0.00 \\
0.020 & 0.00 \\
0.023 & 0.00 \\
0.026 & 0.00 \\
0.030 & 0.00 \\
0.035 & 0.00 \\
0.040 & 0.00 \\
0.046 & 0.00 \\
0.052 & 0.00 \\
0.060 & 0.00 \\
0.069 & 0.00 \\
0.079 & 0.00 \\
0.091 & 0.00 \\
0.105 & \\
\hline
\end{tabular}

\begin{tabular}{|r|r|}
\hline Size $(\mu \mathrm{m})$ & Volume In $\%$ \\
\hline 0.105 & 0.00 \\
0.120 & 0.00 \\
0.138 & 0.00 \\
0.158 & 0.00 \\
0.182 & 0.00 \\
0.209 & 0.00 \\
0.240 & 0.00 \\
0.275 & 0.00 \\
0.316 & 0.00 \\
0.363 & 0.00 \\
0.417 & 0.00 \\
0.479 & 0.00 \\
0.550 & 0.00 \\
0.631 & 0.00 \\
0.724 & 0.00 \\
0.832 & 0.00 \\
0.955 & 0.00 \\
1.096 & \\
\hline
\end{tabular}

\begin{tabular}{|r|r|}
\hline Size $(\mu \mathrm{m})$ & Volume $\ln \%$ \\
\hline 1.096 & 0.00 \\
1.259 & 0.00 \\
1.445 & 0.00 \\
1.660 & 0.00 \\
1.905 & 0.00 \\
2.188 & 0.00 \\
2.512 & 0.00 \\
2.884 & 0.00 \\
3.311 & 0.03 \\
3.802 & 0.08 \\
4.365 & 0.10 \\
5.012 & 0.16 \\
5.754 & 0.22 \\
6.607 & 0.30 \\
7.586 & 0.39 \\
8.710 & 0.48 \\
10.000 & 0.58 \\
11.482 & \\
\hline
\end{tabular}

\begin{tabular}{|r|r|}
\hline Size $(\mu \mathrm{m})$ & Volume $\ln \%$ \\
\hline 11.482 & 0.68 \\
13.183 & 0.79 \\
15.136 & 0.92 \\
17.378 & 1.08 \\
19.953 & 1.29 \\
22.909 & 1.63 \\
26.303 & 2.24 \\
30.200 & 3.30 \\
34.674 & 4.92 \\
39.811 & 6.94 \\
45.709 & 8.94 \\
52.481 & 10.51 \\
60.256 & 11.46 \\
69.183 & 11.81 \\
79.433 & 11.31 \\
91.201 & 9.96 \\
104.713 & 6.22 \\
120.226 & \\
\hline
\end{tabular}

\begin{tabular}{|r|r|}
\hline Size $(\mu \mathrm{m})$ & Volume $\ln \%$ \\
\hline 120.226 & 3.27 \\
138.038 & 0.39 \\
158.489 & 0.00 \\
181.970 & 0.00 \\
208.930 & 0.00 \\
239.883 & 0.00 \\
275.423 & 0.00 \\
316.228 & 0.00 \\
363.078 & 0.00 \\
416.869 & 0.00 \\
478.630 & 0.00 \\
549.541 & 0.00 \\
630.957 & 0.00 \\
724.436 & 0.00 \\
831.764 & 0.00 \\
954.993 & 0.00 \\
1096.478 & 0.00 \\
1258.925 & \\
\hline
\end{tabular}

\begin{tabular}{|r|r|}
\hline Size $(\mu \mathrm{m})$ & Volume $\ln \%$ \\
\hline 1258.925 & 0.00 \\
1445.440 & 0.00 \\
1659.587 & 0.00 \\
1905.461 & 0.00 \\
2187.762 & 0.00 \\
2511.886 & 0.00 \\
2884.032 & 0.00 \\
3311.311 & 0.00 \\
3801.894 & 0.00 \\
4365.158 & 0.00 \\
5011.872 & 0.00 \\
5754.399 & 0.00 \\
6606.934 & 0.00 \\
7585.776 & 0.00 \\
8709.636 & 0.00 \\
10000.000 & \\
& \\
& \\
\hline
\end{tabular}


Figure A.7: Particle size distribution of 75-90 $\mu \mathrm{m}$ coal fraction as measured by the Malvern Mastersizer. $d_{10}=24 \mu \mathrm{m} ; d_{50}=77 \mu \mathrm{m} ; d_{90}=116 \mu \mathrm{m}$

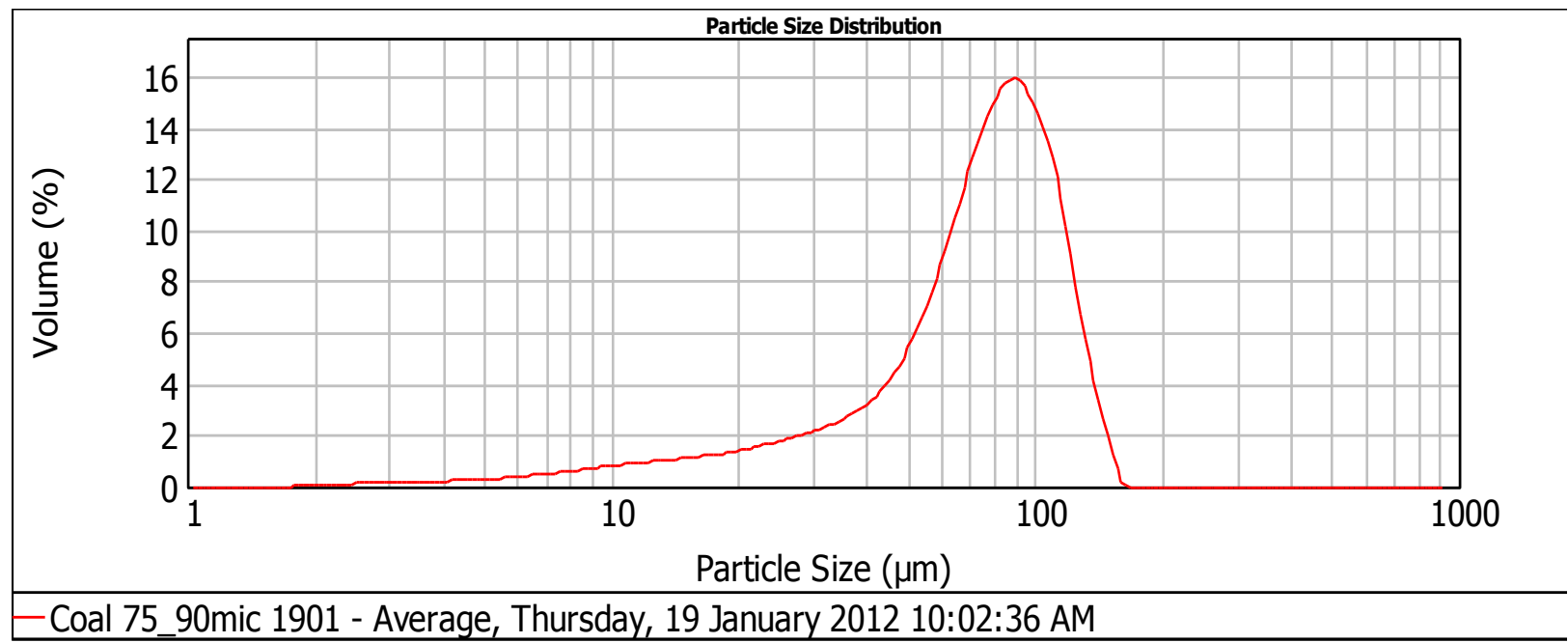

Table A.7: Volume frequency distribution data for 75-90 micron coal as measured by the Malvern Mastersizer.

\begin{tabular}{|r|r|}
\hline Size $(\mu \mathrm{m})$ & Volume In $\%$ \\
\hline 0.010 & 0.00 \\
0.011 & 0.00 \\
0.013 & 0.00 \\
0.015 & 0.00 \\
0.017 & 0.00 \\
0.020 & 0.00 \\
0.023 & 0.00 \\
0.026 & 0.00 \\
0.030 & 0.00 \\
0.035 & 0.00 \\
0.040 & 0.00 \\
0.046 & 0.00 \\
0.052 & 0.00 \\
0.060 & 0.00 \\
0.069 & 0.00 \\
0.079 & 0.00 \\
0.091 & 0.00 \\
0.105 & \\
\hline
\end{tabular}

\begin{tabular}{|r|r|}
\hline Size $(\mu \mathrm{m})$ & Volume $\ln \%$ \\
\hline 0.105 & 0.00 \\
0.120 & 0.00 \\
0.138 & 0.00 \\
0.158 & 0.00 \\
0.182 & 0.00 \\
0.209 & 0.00 \\
0.240 & 0.00 \\
0.275 & 0.00 \\
0.316 & 0.00 \\
0.363 & 0.00 \\
0.417 & 0.00 \\
0.479 & 0.00 \\
0.550 & 0.00 \\
0.631 & 0.00 \\
0.724 & 0.00 \\
0.832 & 0.00 \\
0.955 & 0.00 \\
1.096 & \\
\hline
\end{tabular}

\begin{tabular}{|r|r|}
\hline Size $(\mu \mathrm{m})$ & Volume $\ln \%$ \\
\hline 1.096 & 0.00 \\
1.259 & 0.00 \\
1.445 & 0.00 \\
1.660 & 0.00 \\
1.905 & 0.07 \\
2.188 & 0.09 \\
2.512 & 0.11 \\
2.884 & 0.13 \\
3.311 & 0.15 \\
3.802 & 0.18 \\
4.365 & 0.23 \\
5.012 & 0.28 \\
5.754 & 0.35 \\
6.607 & 0.45 \\
7.586 & 0.55 \\
8.710 & 0.67 \\
10.000 & 0.78 \\
11.482 & \\
\hline
\end{tabular}

\begin{tabular}{|r|r|}
\hline Size $(\mu \mathrm{m})$ & Volume In $\%$ \\
\hline 11.482 & 0.87 \\
13.183 & 0.96 \\
15.136 & 1.05 \\
17.378 & 1.17 \\
19.953 & 1.35 \\
22.909 & 1.57 \\
26.303 & 1.81 \\
30.200 & 2.11 \\
34.674 & 2.53 \\
39.811 & 3.24 \\
45.709 & 4.52 \\
52.481 & 6.57 \\
60.256 & 9.37 \\
69.183 & 12.28 \\
79.433 & 14.22 \\
91.201 & 13.75 \\
104.713 & 11.03 \\
120.226 & \\
\hline
\end{tabular}

\begin{tabular}{|r|r|}
\hline Size $(\mu \mathrm{m})$ & Volume $\ln \%$ \\
\hline 120.226 & 5.68 \\
138.038 & 1.89 \\
158.489 & 0.00 \\
181.970 & 0.00 \\
208.930 & 0.00 \\
239.883 & 0.00 \\
275.423 & 0.00 \\
316.228 & 0.00 \\
363.078 & 0.00 \\
416.869 & 0.00 \\
478.630 & 0.00 \\
549.541 & 0.00 \\
630.957 & 0.00 \\
724.436 & 0.00 \\
831.764 & 0.00 \\
954.993 & 0.00 \\
1096.478 & 0.00 \\
1258.925 & \\
\hline
\end{tabular}

\begin{tabular}{|r|r|}
\hline Size $(\mu \mathrm{m})$ & Volume $\ln \%$ \\
\hline 1258.925 & 0.00 \\
1445.440 & 0.00 \\
1659.587 & 0.00 \\
1905.461 & 0.00 \\
2187.762 & 0.00 \\
2511.886 & 0.00 \\
2884.032 & 0.00 \\
3311.311 & 0.00 \\
3801.894 & 0.00 \\
4365.158 & 0.00 \\
5011.872 & 0.00 \\
5754.399 & 0.00 \\
6606.934 & 0.00 \\
7585.776 & 0.00 \\
8709.636 & 0.00 \\
10000.000 & \\
& \\
& \\
\hline
\end{tabular}


Figure A.8: Particle size distribution of 45-63 $\mu \mathrm{m}$ coal fraction as measured by the Malvern Mastersizer. $d_{10}=30 \mu \mathrm{m} ; d_{50}=52 \mu \mathrm{m} ; d_{90}=78 \mu \mathrm{m}$

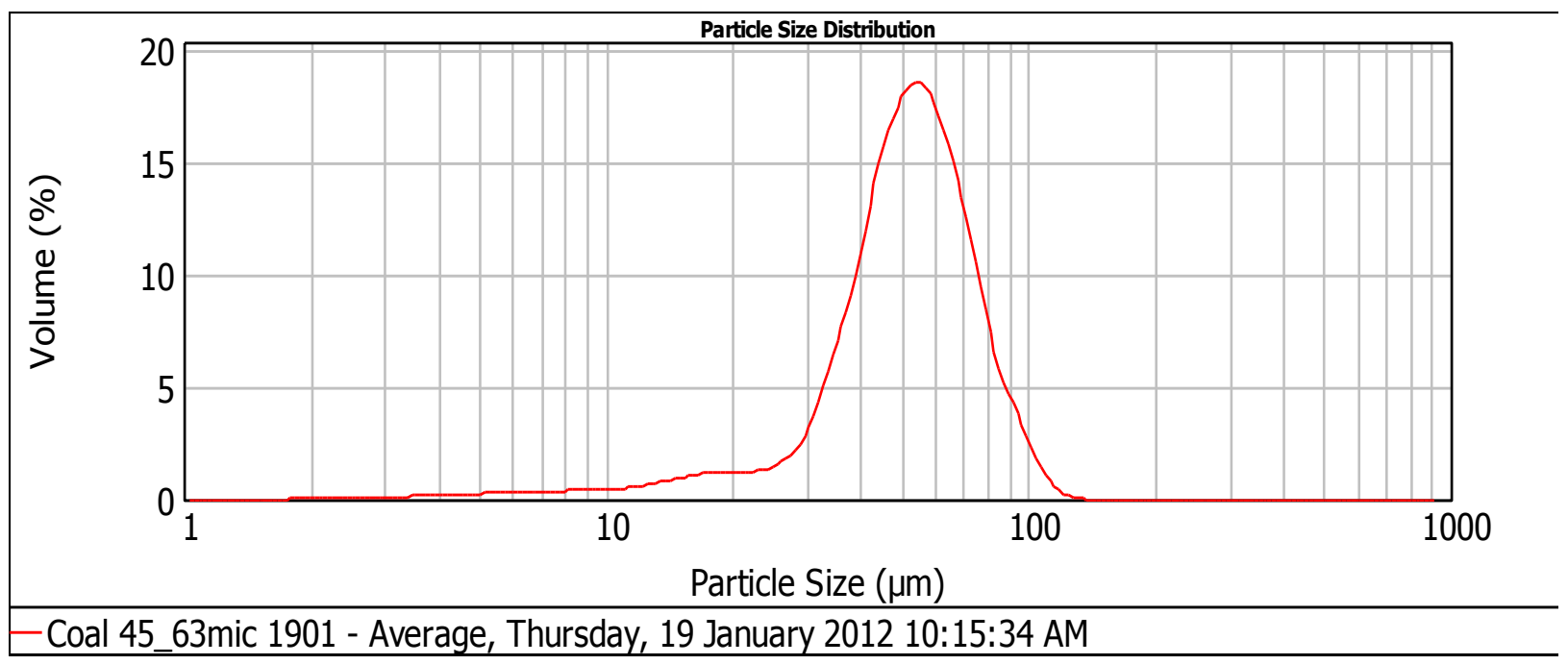

Table A.8: Volume frequency distribution data for 45-63 micron coal as measured by the Malvern Mastersizer.

\begin{tabular}{|c|c|c|c|}
\hline$\overline{\operatorname{Size}(\mu \mathrm{m})}$ & Volume $\ln \%$ & Size $(\mu \mathrm{m})$ & Volume In \% \\
\hline 0.010 & $0 \Omega$ & 0.105 & 000 \\
\hline 0.011 & & 0.120 & 0.00 \\
\hline 0.013 & 0.00 & 0.138 & 0.00 \\
\hline 0.015 & 0.00 & 0158 & 0.00 \\
\hline $0 \Omega 17$ & 0.00 & 0.100 & 0.00 \\
\hline 0.017 & 0.00 & 0.182 & 0,00 \\
\hline 0.020 & 000 & 0.209 & 000 \\
\hline 0.023 & 0.00 & 0.240 & 0.00 \\
\hline 0.026 & 0.00 & 0.275 & 0.00 \\
\hline 0.030 & 0.00 & 0.316 & 0.00 \\
\hline 0.035 & 0.00 & 0.363 & 0.00 \\
\hline 0.040 & 0.00 & 0417 & 0.00 \\
\hline 0.046 & 0.00 & 0170 & 0.00 \\
\hline 0.052 & 0.00 & 0.710 & 0.00 \\
\hline 0.060 & 0.00 & 0021 & 0.00 \\
\hline 0.069 & 0.00 & 0.07 & 0.00 \\
\hline & 0.00 & & 0.00 \\
\hline 0091 & 0.00 & 0.0032 & 0.00 \\
\hline 0.091 & 000 & 0.955 & 000 \\
\hline 0.105 & & 1.096 & \\
\hline
\end{tabular}

\begin{tabular}{|r|r|}
\hline Size $(\mu \mathrm{m})$ & Volume In \% \\
\hline 1.096 & 0.00 \\
1.259 & 0.00 \\
1.445 & 0.00 \\
1.660 & 0.00 \\
1.905 & 0.08 \\
2.188 & 0.08 \\
2.512 & 0.09 \\
2.884 & 0.10 \\
3.311 & 0.12 \\
3.802 & 0.15 \\
4.365 & 0.19 \\
5.012 & 0.24 \\
5.754 & 0.29 \\
6.607 & 0.32 \\
7.586 & 0.34 \\
8.710 & 0.36 \\
10.000 & 0.42 \\
11.482 & \\
\hline
\end{tabular}

\begin{tabular}{|r|r|}
\hline Size $(\mu \mathrm{m})$ & Volume $\ln \%$ \\
\hline 11.482 & 0.55 \\
13.183 & 0.75 \\
15.136 & 0.96 \\
17.378 & 1.09 \\
19.953 & 1.10 \\
22.909 & 1.25 \\
26.303 & 1.96 \\
30.200 & 4.21 \\
34.674 & 7.52 \\
39.811 & 12.25 \\
45.709 & 15.74 \\
52.481 & 16.65 \\
60.256 & 14.26 \\
69.183 & 10.08 \\
79.433 & 5.25 \\
91.201 & 2.79 \\
104.713 & 0.73 \\
120.226 & \\
\hline
\end{tabular}

\begin{tabular}{|r|r|}
\hline Size $(\mu \mathrm{m})$ & Volume $\ln \%$ \\
\hline 120.226 & 0.05 \\
138.038 & 0.00 \\
158.489 & 0.00 \\
181.970 & 0.00 \\
208.930 & 0.00 \\
239.883 & 0.00 \\
275.423 & 0.00 \\
316.228 & 0.00 \\
363.078 & 0.00 \\
416.869 & 0.00 \\
478.630 & 0.00 \\
549.541 & 0.00 \\
630.957 & 0.00 \\
724.436 & 0.00 \\
831.764 & 0.00 \\
954.993 & 0.00 \\
1096.478 & 0.00 \\
1258.925 & \\
\hline
\end{tabular}

\begin{tabular}{|r|r|}
\hline Size $(\mu \mathrm{m})$ & Volume $\ln \%$ \\
\hline 1258.925 & 0.00 \\
1445.440 & 0.00 \\
1659.587 & 0.00 \\
1905.461 & 0.00 \\
2187.762 & 0.00 \\
2511.886 & 0.00 \\
2884.032 & 0.00 \\
3311.311 & 0.00 \\
3801.894 & 0.00 \\
4365.158 & 0.00 \\
5011.872 & 0.00 \\
5754.399 & 0.00 \\
6606.934 & 0.00 \\
7585.776 & 0.00 \\
8709.636 & 0.00 \\
10000.000 & \\
& \\
\hline
\end{tabular}




\section{APPENDIX B}

\section{Calibration Curve of Water Rotameter}

Water flow rate was measured using a beaker and stopwatch for a range of flows from 20 to $100 \%$ of scale. The results are plotted in Figure B.1. The trend is well fitted by a straight line through the origin.

Table B.1: Water rotameter calibration measurements.

\begin{tabular}{cccc}
\hline $\begin{array}{c}\text { Scale Reading } \\
(\%)\end{array}$ & $\begin{array}{c}\text { Volume } \\
\text { (mL) }\end{array}$ & $\begin{array}{c}\text { Time } \\
\text { (s) }\end{array}$ & $\begin{array}{c}\text { Vol. Rate } \\
\text { (L/min) }\end{array}$ \\
\hline $20 \%$ & 500 & 69.0 & 0.435 \\
$30 \%$ & 425 & 44.6 & 0.571 \\
$40 \%$ & 500 & 35.8 & 0.837 \\
$50 \%$ & 420 & 25.1 & 1.003 \\
$50 \%$ & 467 & 27.9 & 1.006 \\
$60 \%$ & 490 & 24.3 & 1.209 \\
$70 \%$ & 420 & 18.7 & 1.349 \\
$74 \%$ & 480 & 19.4 & 1.487 \\
$100 \%$ & 455 & 14.7 & 1.858 \\
\hline
\end{tabular}

\section{G4 Meter-1/2-GSVT-45 Heavier Float}

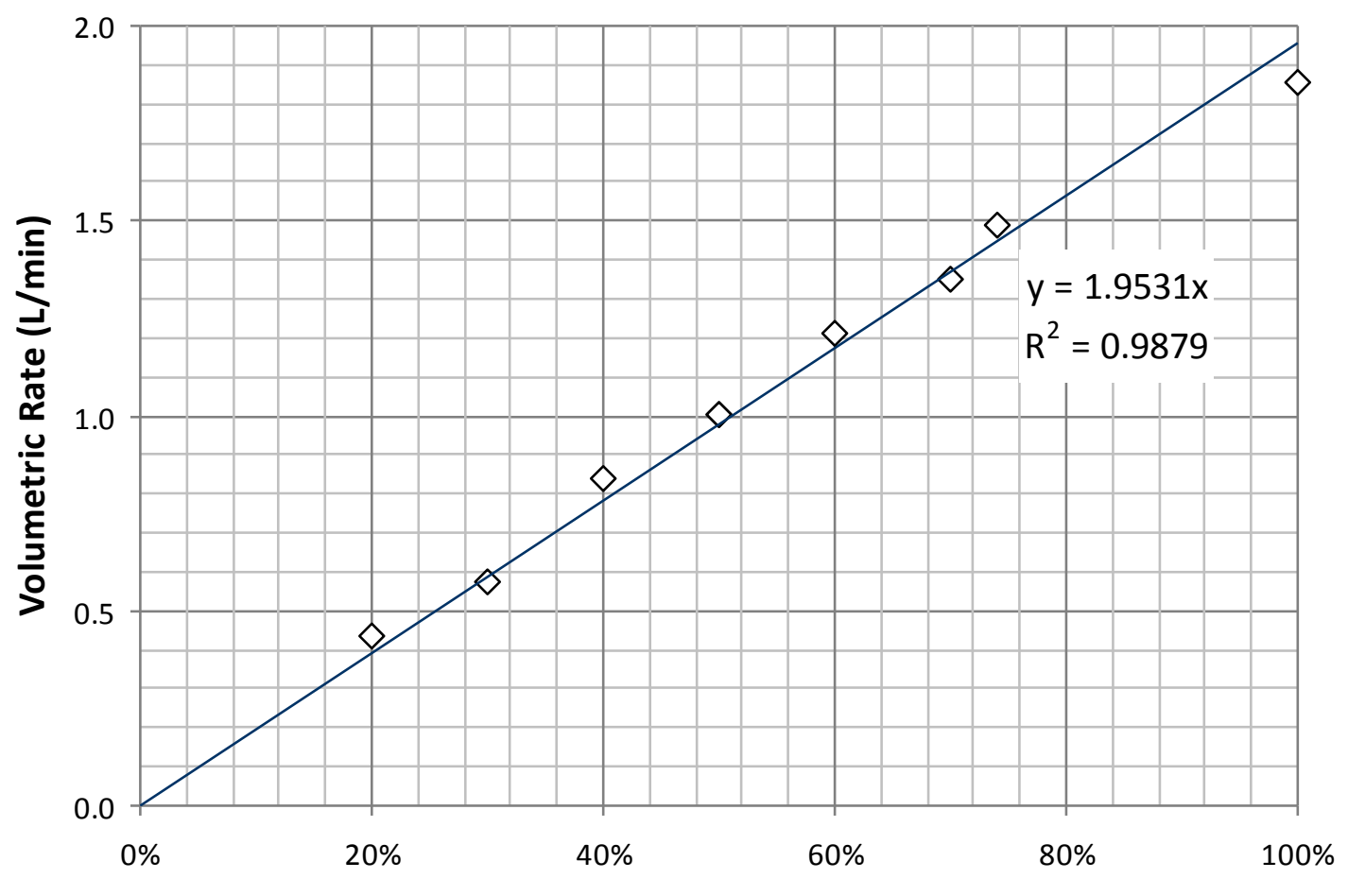

Flow Rate \%

Figure B.1: Water rotameter calibration chart. 


\section{APPENDIX C}

\section{Drop Diameter Calculation of Multiple Drop Experimental System.}

\begin{tabular}{|c|c|c|c|c|c|}
\hline \multicolumn{5}{|c|}{ Average no. of drops per 10 seconds per needle } & \\
\hline Time (S) & $\begin{array}{l}\text { Total Volume } \\
\left(\mathrm{ml} \mathrm{or} \mathrm{cm}^{3}\right)\end{array}$ & $\begin{array}{l}\text { Total } \\
\text { Drops }\end{array}$ & $\begin{array}{l}\text { Volume of a drop } \\
\left(\mathrm{cm}^{3}\right)\end{array}$ & $\begin{array}{l}\text { Radius of a } \\
\text { drop }(\mathrm{cm})\end{array}$ & Diameter of a drop $(\mathrm{cm})$ \\
\hline 3480 & 198 & 10440 & 0.018965517 & 0.001509993 & 0.003019987 \\
\hline 3480 & 200 & 10440 & 0.019157088 & 0.001525246 & 0.003050492 \\
\hline 3090 & 180 & 9270 & 0.019417476 & 0.001545977 & 0.003091955 \\
\hline
\end{tabular}

Average drop diameter $=0.00305 \mathrm{~cm}$

$$
=\underline{3.05 \mathrm{~mm}}
$$

\section{Terminal Velocity Calculation of Water Drops}

Assuming there is no pressure in the needle;

$$
\mathrm{v}=\mathrm{u}+\mathrm{at}
$$

Since $u=0$;

$\mathrm{v}=9.81^{*}(0.1427 / 2)$

$\mathrm{v}=1.399 \mathrm{~ms}^{-1}$

$\mathrm{v}=1.4 \mathrm{~ms}^{-1}$ 


\section{APPENDIX D}

\section{All Experimental Results of Multi Drop Experimental System}

1. Recovery of ballotini as received $23-47 \mu \mathrm{m}\left(\mathrm{d}_{50}=33 \mu \mathrm{m}\right)$ with tap water.

\begin{tabular}{|c|c|c|c|c|c|c|c|c|c|c|}
\hline $\begin{array}{c}\text { Air } \\
\text { Flowr } \\
\text { ate } \\
(\%)\end{array}$ & $\begin{array}{c}\text { Water } \\
\text { Flowrate } \\
(\%)\end{array}$ & $\begin{array}{c}\text { Water } \\
\text { Volumetric } \\
\text { Rate } \\
(\mathrm{L} / \mathrm{min})\end{array}$ & $\begin{array}{c}\text { Water } \\
\text { Superficial } \\
\text { Velocity } \\
(\mathrm{m} / \mathrm{s})\end{array}$ & $\begin{array}{c}\text { Total } \\
\text { Feed } \\
(\mathrm{g})\end{array}$ & $\begin{array}{c}\text { Mas in } \\
\text { Cyclone } \\
(\mathrm{g})\end{array}$ & $\begin{array}{c}\text { Mass in } \\
2^{\text {nd }} \\
\text { Chamber } \\
(\mathrm{g})\end{array}$ & $\begin{array}{c}\text { Underflow } \\
\text { Mass } \\
(\mathrm{g})\end{array}$ & $\begin{array}{c}\text { Total } \\
\text { Mass } \\
\text { Recovered } \\
(\mathrm{g})\end{array}$ & $\begin{array}{c}\text { Recovery } \\
\text { to } \\
\text { Underflow } \\
(\mathrm{wt} \%)\end{array}$ & $\begin{array}{c}\text { Experime } \\
\text { ntal } \\
\text { Losses } \\
(\mathrm{wt} \%)\end{array}$ \\
\hline 40 & 0 & 0 & 0 & 49.7 & 44.4 & 2 & 1.7 & 48.1 & 3.53 & 3.22 \\
\hline 40 & 30 & 0.65 & 0.0057 & 97.0 & 82.7 & 2.3 & 10.1 & 95.1 & 10.6 & 2.0 \\
\hline 40 & 50 & 1 & 0.0088 & 97.1 & 67.7 & 4.7 & 13.8 & 86.2 & 16.0 & 11.2 \\
\hline 40 & 70 & 1.35 & 0.0118 & 98.3 & 54.7 & 6.7 & 17.3 & 78.7 & 22.0 & 19.9 \\
\hline 40 & 70 & 1.35 & 0.0118 & 98.3 & 74.8 & 1.3 & 17.1 & 93.2 & 18.3 & 5.2 \\
\hline 40 & 85 & 1.63 & 0.0143 & 99.0 & 74.5 & 1 & 23.5 & 99.0 & 23.7 & 0.0 \\
\hline 40 & 100 & 1.9 & 0.0167 & 99.0 & 64.0 & 1.2 & 31.1 & 96.3 & 32.3 & 2.7 \\
\hline 40 & 100 & 1.9 & 0.0167 & 99.1 & 59.5 & 4.7 & 31.1 & 95.3 & 32.6 & 3.8 \\
\hline
\end{tabular}

2. Recovery predicted from Analytical model (For $u_{p} \gg u_{d}$ )

\begin{tabular}{|l|l|l|l|l|l|}
\hline $\begin{array}{l}\text { Water } \\
\text { Flowrate }(\%)\end{array}$ & $\begin{array}{l}\text { Water } \\
\text { Superficial } \\
\text { Velocity-W } \\
(\mathrm{m} / \mathrm{s})\end{array}$ & $\begin{array}{l}\text { Drop } \\
\text { Diameter }-\mathrm{D}_{\mathrm{d}} \\
(\mathrm{m})\end{array}$ & $\begin{array}{l}\text { Drop } \\
\text { Velocity- } \mathrm{U}_{\mathrm{d}} \\
(\mathrm{m} / \mathrm{s})\end{array}$ & $\begin{array}{l}\text { Length of } \\
\text { water array-X } \\
(\mathrm{m})\end{array}$ & $\begin{array}{l}\text { Underflow } \\
\text { Recovery }(\%)\end{array}$ \\
\hline 0 & 0 & 0.003 & 1.04 & 0.0246 & 0 \\
\hline 30 & 0.0057 & 0.003 & 1.04 & 0.0246 & 6.74 \\
\hline 50 & 0.0088 & 0.003 & 1.04 & 0.0246 & 10.41 \\
\hline 70 & 0.0118 & 0.003 & 1.04 & 0.0246 & 13.96 \\
\hline 85 & 0.0143 & 0.003 & 1.04 & 0.0246 & 16.91 \\
\hline 100 & 0.0167 & 0.003 & 1.04 & 0.0246 & 19.75 \\
\hline
\end{tabular}

3. Recovery predicted from DEM

\begin{tabular}{|c|c|c|c|c|}
\hline $\mathrm{Up}(\mathrm{m} / \mathrm{s})$ & Water Flux $(\mathrm{m} / \mathrm{s})$ & Particles Captured & $\begin{array}{l}\text { Particles Not } \\
\text { Captured }\end{array}$ & $\%$ Captured \\
\hline 6 & 0.0101 & 3841 & 29448 & 11.538 \\
\hline 6 & 0.0112 & 4191 & 29169 & 12.563 \\
\hline 6 & 0.0126 & 4728 & 28937 & 14.044 \\
\hline 6 & 0.0145 & 5527 & 28500 & 16.243 \\
\hline
\end{tabular}


4. Recovery of cleaned glass ballotini $23-47 \mu \mathrm{m}\left(\mathrm{d}_{50}=33 \mu \mathrm{m}\right)$ with tap water.

\begin{tabular}{|c|c|c|c|c|c|c|c|c|c|c|}
\hline $\begin{array}{c}\text { Air } \\
\text { Flowrate } \\
(\%)\end{array}$ & $\begin{array}{c}\text { Water } \\
\text { Flowrate } \\
(\%)\end{array}$ & $\begin{array}{c}\text { Water } \\
\text { Volumetric } \\
\text { Rate } \\
(\mathrm{L} / \mathrm{min})\end{array}$ & $\begin{array}{c}\text { Water } \\
\text { Superficial } \\
\text { Velocity } \\
(\mathrm{m} / \mathrm{s})\end{array}$ & $\begin{array}{c}\text { Total } \\
\text { Feed } \\
(\mathrm{g})\end{array}$ & $\begin{array}{c}\text { Mas in } \\
\text { Cyclone } \\
(\mathrm{g})\end{array}$ & $\begin{array}{c}\text { Mass in } \\
2^{\text {nd }} \\
\text { Chamber } \\
(\mathrm{g})\end{array}$ & $\begin{array}{c}\text { Underflow } \\
\text { Mass } \\
(\mathrm{g})\end{array}$ & $\begin{array}{c}\text { Total } \\
\text { Mass } \\
\text { Recovered } \\
(\mathrm{g})\end{array}$ & $\begin{array}{c}\text { Recovery } \\
\text { to } \\
\text { Underflow } \\
(\mathrm{wt} \%)\end{array}$ & $\begin{array}{c}\text { Experimental } \\
\text { Losses } \\
(\mathrm{wt} \%)\end{array}$ \\
\hline 40 & 0 & 0 & 0 & 49.7 & 44.4 & 2 & 1.7 & 48.1 & 3.5 & 3.2 \\
\hline 43 & 30 & 0.65 & 0.0057 & 87.4 & 70.2 & 2.1 & 10.9 & 83.2 & 13.1 & 4.8 \\
\hline 40 & 50 & 1.00 & 0.0088 & 98.8 & 55.7 & 2.7 & 14.3 & 72.7 & 19.7 & 26.4 \\
\hline 43 & 50 & 1.00 & 0.0088 & 98.4 & 68.8 & 1.3 & 21.3 & 91.4 & 23.3 & 7.1 \\
\hline 42 & 70 & 1.35 & 0.0118 & 91.3 & 52.6 & 2.1 & 24.4 & 79.1 & 30.8 & 13.4 \\
\hline 40 & 100 & 1.35 & 0.0118 & 98.1 & 39.5 & 2.4 & 27.1 & 69.0 & 39.3 & 29.7 \\
\hline 42 & 100 & 1.90 & 0.0167 & 98.2 & 47.9 & 1.9 & 30.5 & 80.3 & 38.0 & 18.2 \\
\hline 41 & 100 & 1.90 & 0.0167 & 95.2 & 58.2 & 1.1 & 30.4 & 89.7 & 33.9 & 5.8 \\
\hline
\end{tabular}

5. Recovery of cleaned glass ballotini $17-36 \mu \mathrm{m}\left(\mathrm{d}_{50}=25 \mu \mathrm{m}\right)$ with tap water.

\begin{tabular}{|c|c|c|c|c|c|c|c|c|c|c|}
\hline $\begin{array}{c}\text { Air } \\
\text { Flowrate } \\
(\%)\end{array}$ & $\begin{array}{c}\text { Water } \\
\text { Flowrate } \\
(\%)\end{array}$ & $\begin{array}{c}\text { Water } \\
\text { Volumetric } \\
\text { Rate } \\
(\mathrm{L} / \mathrm{min})\end{array}$ & $\begin{array}{c}\text { Water } \\
\text { Superficial } \\
\text { Velocity } \\
(\mathrm{m} / \mathrm{s})\end{array}$ & $\begin{array}{c}\text { Total } \\
\text { Feed } \\
(\mathrm{g})\end{array}$ & $\begin{array}{c}\text { Mas in } \\
\text { Cyclone } \\
(\mathrm{g})\end{array}$ & $\begin{array}{c}\text { Mass in } \\
2^{\text {nd }} \\
\text { Chamber } \\
(\mathrm{g})\end{array}$ & $\begin{array}{c}\text { Underflow } \\
\text { Mass } \\
(\mathrm{g})\end{array}$ & $\begin{array}{c}\text { Total } \\
\text { Mass } \\
\text { Recovered } \\
(\mathrm{g})\end{array}$ & $\begin{array}{c}\text { Recovery } \\
\text { to } \\
\text { Underflow } \\
(\mathrm{wt} \%)\end{array}$ & $\begin{array}{c}\text { Experimental } \\
\text { Losses } \\
(\mathrm{wt} \%)\end{array}$ \\
\hline 40 & 0 & 0 & 0 & 49.3 & 39.7 & 3.1 & 2.1 & 44.9 & 4.7 & 8.9 \\
\hline 42 & 30 & 0.65 & 0.0057 & 48.5 & 39.9 & 1.1 & 6.7 & 47.7 & 14.0 & 1.6 \\
\hline 40 & 50 & 1.00 & 0.0088 & 49.2 & 40.1 & 0.8 & 7.0 & 47.9 & 14.6 & 2.6 \\
\hline 35 & 60 & 1.19 & 0.0104 & 48.6 & 28.7 & 2.1 & 5.2 & 36.0 & 14.4 & 25.9 \\
\hline 35 & 70 & 1.35 & 0.0118 & 48.7 & 26.6 & 0.6 & 5.9 & 33.1 & 17.8 & 32.0 \\
\hline 42 & 70 & 1.35 & 0.0118 & 48.6 & 32.3 & 0.9 & 9 & 42.2 & 21.3 & 13.2 \\
\hline 42 & 70 & 1.35 & 0.0118 & 48.9 & 34.7 & 0.7 & 7.6 & 43.0 & 17.7 & 12.2 \\
\hline 40 & 85 & 1.63 & 0.0143 & 48.2 & 31.0 & 1.2 & 11 & 43.2 & 25.5 & 10.4 \\
\hline 40 & 100 & 1.90 & 0.0167 & 49.0 & 30.3 & 0.8 & 15.8 & 46.9 & 33.7 & 4.3 \\
\hline
\end{tabular}

6. Recovery of cleaned rough silica flour $8-29 \mu \mathrm{m}\left(\mathrm{d}_{50}=16 \mu \mathrm{m}\right)$ with tap water.

\begin{tabular}{|c|c|c|c|c|c|c|c|c|c|c|}
\hline $\begin{array}{c}\text { Air } \\
\text { Flowrate } \\
(\%)\end{array}$ & $\begin{array}{c}\text { Water } \\
\text { Flowrate } \\
(\%)\end{array}$ & $\begin{array}{c}\text { Water } \\
\text { Volumetric } \\
\text { Rate } \\
(\mathrm{L} / \mathrm{min})\end{array}$ & $\begin{array}{c}\text { Water } \\
\text { Superficial } \\
\text { Velocity } \\
(\mathrm{m} / \mathrm{s})\end{array}$ & $\begin{array}{c}\text { Total } \\
\text { Feed } \\
(\mathrm{g})\end{array}$ & $\begin{array}{c}\text { Mas in } \\
\text { Cyclone } \\
(\mathrm{g})\end{array}$ & $\begin{array}{c}\text { Mass in } \\
2^{\text {nd }} \\
\text { Chamber } \\
(\mathrm{g})\end{array}$ & $\begin{array}{c}\text { Underflow } \\
\text { Mass } \\
(\mathrm{g})\end{array}$ & $\begin{array}{c}\text { Total } \\
\text { Mass } \\
\text { Recovered } \\
(\mathrm{g})\end{array}$ & $\begin{array}{c}\text { Recovery } \\
\text { to } \\
\text { Underflow } \\
(\mathrm{wt} \%)\end{array}$ & $\begin{array}{c}\text { Experimental } \\
\text { Losses } \\
(\mathrm{wt} \%)\end{array}$ \\
\hline 40 & 50 & 1.00 & 0.0088 & 49.3 & 31.1 & 2.6 & 11.9 & 46.2 & 25.76 & 6.29 \\
\hline 40 & 85 & 1.63 & 0.0143 & 49.5 & 28.5 & 2.1 & 14.3 & 45 & 31.78 & 9.09 \\
\hline
\end{tabular}


7. Recovery of cleaned rough silica flour $16-42 \mu \mathrm{m}\left(\mathrm{d}_{50}=27 \mu \mathrm{m}\right)$ with tap water.

\begin{tabular}{|c|c|c|c|c|c|c|c|c|c|c|}
\hline $\begin{array}{c}\text { Air } \\
\begin{array}{c}\text { Flowrate } \\
(\%)\end{array}\end{array}$ & $\begin{array}{c}\text { Water } \\
\text { Flowrate } \\
(\%)\end{array}$ & $\begin{array}{c}\text { Water } \\
\text { Volumetric } \\
\text { Rate } \\
(\mathrm{L} / \mathrm{min})\end{array}$ & $\begin{array}{c}\text { Water } \\
\text { Superficial } \\
\text { Velocity } \\
(\mathrm{m} / \mathrm{s})\end{array}$ & $\begin{array}{c}\text { Total } \\
\text { Feed } \\
(\mathrm{g})\end{array}$ & $\begin{array}{c}\text { Mas in } \\
\text { Cyclone } \\
(\mathrm{g})\end{array}$ & $\begin{array}{c}\text { Mass in } \\
2^{\text {nd }} \\
\text { Chamber } \\
(\mathrm{g})\end{array}$ & $\begin{array}{c}\text { Underflow } \\
\text { Mass } \\
(\mathrm{g})\end{array}$ & $\begin{array}{c}\text { Total } \\
\text { Mass } \\
\text { Recovered } \\
(\mathrm{g})\end{array}$ & $\begin{array}{c}\text { Recovery } \\
\text { to } \\
\text { Underflow } \\
(\mathrm{wt} \%)\end{array}$ & $\begin{array}{c}\text { Experimental } \\
\text { Losses } \\
(\mathrm{wt} \%)\end{array}$ \\
\hline 40 & 50 & 1.00 & 0.0088 & 49.3 & 34.2 & 2.8 & 10.8 & 48.2 & 22.41 & 2.23 \\
\hline 40 & 85 & 1.63 & 0.0143 & 49.1 & 31.8 & 2.4 & 14.8 & 49.2 & 30.08 & -0.20 \\
\hline
\end{tabular}

8. Recovery of cleaned rough silica flour $21-54 \mu \mathrm{m}\left(\mathrm{d}_{50}=34 \mu \mathrm{m}\right)$ with tap water

\begin{tabular}{|c|c|c|c|c|c|c|c|c|c|c|}
\hline $\begin{array}{c}\text { Air } \\
\text { Flowrate } \\
(\%)\end{array}$ & $\begin{array}{c}\text { Water } \\
\text { Flowrate } \\
(\%)\end{array}$ & $\begin{array}{c}\text { Water } \\
\text { Volumetric } \\
\text { Rate } \\
(\mathrm{L} / \mathrm{min})\end{array}$ & $\begin{array}{c}\text { Water } \\
\text { Superficial } \\
\text { Velocity } \\
(\mathrm{m} / \mathrm{s})\end{array}$ & $\begin{array}{c}\text { Total } \\
\text { Feed } \\
(\mathrm{g})\end{array}$ & $\begin{array}{c}\text { Mas in } \\
\text { Cyclone } \\
(\mathrm{g})\end{array}$ & $\begin{array}{c}\text { Mass in } \\
2^{\text {nd }} \\
\text { Chamber } \\
(\mathrm{g})\end{array}$ & $\begin{array}{c}\text { Underflow } \\
\text { Mass } \\
(\mathrm{g})\end{array}$ & $\begin{array}{c}\text { Total } \\
\text { Mass } \\
\text { Recovered } \\
(\mathrm{g})\end{array}$ & $\begin{array}{c}\text { Recovery } \\
\text { to } \\
\text { Underflow } \\
(\mathrm{wt} \%)\end{array}$ & $\begin{array}{c}\text { Experimental } \\
\text { Losses } \\
(\mathrm{wt} \%)\end{array}$ \\
\hline 40 & 0 & 0 & 0 & 49.8 & 40.1 & 5.7 & 0 & 45.8 & 0 & 8.03 \\
\hline 40 & 50 & 1.00 & 0.0088 & 49.8 & 29.4 & 5.6 & 11.9 & 46.9 & 25.37 & 5.82 \\
\hline 40 & 70 & 1.35 & 0.0118 & 49.9 & 31.8 & 4.4 & 13.8 & 50 & 27.60 & -0.02 \\
\hline 40 & 85 & 1.63 & 0.0143 & 49.8 & 26.6 & 5.2 & 15.5 & 47.3 & 32.77 & 5.02 \\
\hline 40 & 85 & 1.63 & 0.0143 & 49.9 & 27.6 & 5.2 & 15.6 & 48.4 & 32.23 & 3.01 \\
\hline
\end{tabular}

9. Recovery of coal $30-78 \mu \mathrm{m}\left(\mathrm{d}_{50}=52 \mu \mathrm{m}\right)$ with tap water

\begin{tabular}{|c|c|c|c|c|c|c|c|c|c|c|}
\hline $\begin{array}{c}\text { Air } \\
\begin{array}{c}\text { Flowrate } \\
(\%)\end{array}\end{array}$ & $\begin{array}{c}\text { Water } \\
\text { Flowrate } \\
(\%)\end{array}$ & $\begin{array}{c}\text { Water } \\
\text { Volumetric } \\
\text { Rate } \\
(\mathrm{L} / \mathrm{min})\end{array}$ & $\begin{array}{c}\text { Water } \\
\text { Superficial } \\
\text { Velocity } \\
(\mathrm{m} / \mathrm{s})\end{array}$ & $\begin{array}{c}\text { Total } \\
\text { Feed } \\
(\mathrm{g})\end{array}$ & $\begin{array}{c}\text { Mas in } \\
\text { Cyclone } \\
(\mathrm{g})\end{array}$ & $\begin{array}{c}\text { Mass in } \\
2^{\text {nd }} \\
\text { Chamber } \\
(\mathrm{g})\end{array}$ & $\begin{array}{c}\text { Underflow } \\
\text { Mass } \\
(\mathrm{g})\end{array}$ & $\begin{array}{c}\text { Total } \\
\text { Mass } \\
\text { Recovered } \\
(\mathrm{g})\end{array}$ & $\begin{array}{c}\text { Recovery } \\
\text { to } \\
\text { Underflow } \\
(\mathrm{wt} \%)\end{array}$ & $\begin{array}{c}\text { Experimental } \\
\text { Losses } \\
(\mathrm{wt} \%)\end{array}$ \\
\hline 40 & 50 & 1 & 0.0088 & 49.6 & 36.6 & 3 & 7.5 & 47.1 & 15.9 & 5.0 \\
\hline 40 & 70 & 1.35 & 0.0118 & 49.4 & 33.0 & 5.1 & 10.3 & 48.4 & 21.3 & 2.0 \\
\hline
\end{tabular}

10. Recovery of coal 29-105 $\mu \mathrm{m}\left(\mathrm{d}_{50}=64 \mu \mathrm{m}\right)$ with tap water

\begin{tabular}{|c|c|c|c|c|c|c|c|c|c|c|}
\hline $\begin{array}{c}\text { Air } \\
\text { Flowrate } \\
(\%)\end{array}$ & $\begin{array}{c}\text { Water } \\
\text { Flowrate } \\
(\%)\end{array}$ & $\begin{array}{c}\text { Water } \\
\text { Volumetric } \\
\text { Rate } \\
(\mathrm{L} / \mathrm{min})\end{array}$ & $\begin{array}{c}\text { Water } \\
\text { Superficial } \\
\text { Velocity } \\
(\mathrm{m} / \mathrm{s})\end{array}$ & $\begin{array}{c}\text { Total } \\
\text { Feed } \\
(\mathrm{g})\end{array}$ & $\begin{array}{c}\text { Mas in } \\
\text { Cyclone } \\
(\mathrm{g})\end{array}$ & $\begin{array}{c}\text { Mass in } \\
2^{\text {nd }} \\
\text { Chamber } \\
(\mathrm{g})\end{array}$ & $\begin{array}{c}\text { Underflow } \\
\text { Mass } \\
(\mathrm{g})\end{array}$ & $\begin{array}{c}\text { Total } \\
\text { Mass } \\
\text { Recovered } \\
(\mathrm{g})\end{array}$ & $\begin{array}{c}\text { Recovery } \\
\text { to } \\
\text { Underflow } \\
(\mathrm{wt} \%)\end{array}$ & $\begin{array}{c}\text { Experimental } \\
\text { Losses } \\
(\mathrm{wt} \%)\end{array}$ \\
\hline 40 & 30 & 0.65 & 0.0057 & 39.0 & 31.9 & 2.3 & 4.5 & 38.7 & 11.6 & \\
\hline 40 & 50 & 1.00 & 0.0088 & 49.4 & 38.6 & 3.7 & 6.8 & 49.1 & 13.8 & 0.8 \\
\hline 40 & 70 & 1.35 & 0.0118 & 47.9 & 36.8 & 2.0 & 6.4 & 45.2 & 14.2 & 5.6 \\
\hline 40 & 70 & 1.35 & 0.0118 & 48.7 & 37.9 & 1.6 & 8.6 & 48.1 & 17.9 & 1.2 \\
\hline 40 & 85 & 1.63 & 0.0143 & 49.7 & 36.6 & 0.3 & 10.9 & 47.8 & 22.8 & 3.8 \\
\hline 40 & 85 & 1.63 & 0.0143 & 49.2 & 32.4 & 4.5 & 10.9 & 47.8 & 22.8 & 2.8 \\
\hline
\end{tabular}


11.Recovery of coal $24-116 \mu \mathrm{m}\left(\mathrm{d}_{50}=77 \mu \mathrm{m}\right)$ with tap water

\begin{tabular}{|c|c|c|c|c|c|c|c|c|c|c|}
\hline $\begin{array}{c}\text { Air } \\
\text { Flowrate } \\
(\%)\end{array}$ & $\begin{array}{c}\text { Fater } \\
\text { Flowrate } \\
(\%)\end{array}$ & $\begin{array}{c}\text { Water } \\
\text { Volumetric } \\
\text { Rate } \\
(\mathrm{L} / \mathrm{min})\end{array}$ & $\begin{array}{c}\text { Water } \\
\text { Superficial } \\
\text { Velocity } \\
(\mathrm{m} / \mathrm{s})\end{array}$ & $\begin{array}{c}\text { Total } \\
\text { Feed } \\
(\mathrm{g})\end{array}$ & $\begin{array}{c}\text { Mas in } \\
\text { Cyclone } \\
(\mathrm{g})\end{array}$ & $\begin{array}{c}\text { Mass in } \\
2^{\text {nd }} \\
\text { Chamber } \\
(\mathrm{g})\end{array}$ & $\begin{array}{c}\text { Underflow } \\
\text { Mass } \\
(\mathrm{g})\end{array}$ & $\begin{array}{c}\text { Total } \\
\text { Mass } \\
\text { Recovered } \\
(\mathrm{g})\end{array}$ & $\begin{array}{c}\text { Recovery } \\
\text { to } \\
\text { Underflow } \\
(\mathrm{wt} \%)\end{array}$ & $\begin{array}{c}\text { Experimental } \\
\text { Losses } \\
(\mathrm{wt} \%)\end{array}$ \\
\hline 40 & 50 & 1 & 0.088 & 49.3 & 37.0 & 2.3 & 7.8 & 47.1 & 16.6 & 4.5 \\
\hline 40 & 70 & 1.35 & 0.0118 & 49.1 & 29.0 & 5.4 & 7.5 & 41.9 & 17.9 & 14.7 \\
\hline 40 & 70 & 1.35 & 0.0118 & 49.6 & 37.9 & 2.6 & 8.2 & 48.7 & 16.8 & 1.8 \\
\hline 40 & 85 & 1.63 & 0.0143 & 49.8 & 32.0 & 4.1 & 11.7 & 47.8 & 24.5 & 4.0 \\
\hline
\end{tabular}

12.Recovery of 21- $54 \mu \mathrm{m}$ cleaned silica with airflow rate of $1.4 \mathrm{~m} / \mathrm{s}$ (tap water)

\begin{tabular}{|c|c|c|c|c|c|c|c|c|c|c|}
\hline $\begin{array}{c}\text { Air } \\
\text { Flowrate } \\
(\%)\end{array}$ & $\begin{array}{c}\text { Water } \\
\text { Flowrate } \\
(\%)\end{array}$ & $\begin{array}{c}\text { Water } \\
\text { Volumetric } \\
\text { Rate } \\
(\mathrm{L} / \mathrm{min})\end{array}$ & $\begin{array}{c}\text { Water } \\
\text { Superficial } \\
\text { Velocity } \\
(\mathrm{m} / \mathrm{s})\end{array}$ & $\begin{array}{c}\text { Total } \\
\text { Feed } \\
(\mathrm{g})\end{array}$ & $\begin{array}{c}\text { Mas in } \\
\text { Cyclone } \\
(\mathrm{g})\end{array}$ & $\begin{array}{c}\text { Mass in } \\
2^{\text {nd }} \\
\text { Chamber } \\
(\mathrm{g})\end{array}$ & $\begin{array}{c}\text { Underflow } \\
\text { Mass } \\
(\mathrm{g})\end{array}$ & $\begin{array}{c}\text { Total } \\
\text { Mass } \\
\text { Recovered } \\
(\mathrm{g})\end{array}$ & $\begin{array}{c}\text { Recovery } \\
\text { to } \\
\text { Underflow } \\
(\mathrm{wt} \%)\end{array}$ & $\begin{array}{c}\text { Experimental } \\
\text { Losses } \\
(\mathrm{wt} \%)\end{array}$ \\
\hline 40 & 50 & 1.00 & 0.0088 & 49.8 & 29.4 & 5.6 & 11.9 & 46.9 & 25.37 & 5.82 \\
\hline 40 & 70 & 1.35 & 0.0118 & 49.9 & 31.8 & 4.4 & 13.8 & 50 & 27.60 & -0.02 \\
\hline 40 & 85 & 1.63 & 0.0143 & 49.8 & 26.6 & 5.2 & 15.5 & 47.3 & 32.77 & 5.02 \\
\hline 40 & 85 & 1.63 & 0.0143 & 49.9 & 27.6 & 5.2 & 15.6 & 48.4 & 32.23 & 3.01 \\
\hline
\end{tabular}

13. Recovery of $21-54 \mu \mathrm{m}\left(\mathrm{d}_{50}=34 \mu \mathrm{m}\right)$ cleaned silica with airflow rate $1.75 \mathrm{~m} / \mathrm{s}$ (with tap water)

\begin{tabular}{|c|c|c|c|c|c|c|c|c|c|c|}
\hline $\begin{array}{c}\text { Air } \\
\text { Flowrate } \\
(\%)\end{array}$ & $\begin{array}{c}\text { Flowrate } \\
(\mathrm{m} / \mathrm{s})\end{array}$ & $\begin{array}{c}\text { Water } \\
\text { Flowrate } \\
(\%)\end{array}$ & $\begin{array}{c}\text { Water } \\
\text { Superficial } \\
\text { Velocity } \\
(\mathrm{m} / \mathrm{s})\end{array}$ & $\begin{array}{c}\text { Total } \\
\text { Feed } \\
(\mathrm{g})\end{array}$ & $\begin{array}{c}\text { Mas in } \\
\text { Cyclone } \\
(\mathrm{g})\end{array}$ & $\begin{array}{c}\text { Mass in } \\
2^{\text {nd }} \\
\text { Chamber } \\
(\mathrm{g})\end{array}$ & $\begin{array}{c}\text { Underflow } \\
\text { Mass } \\
(\mathrm{g})\end{array}$ & $\begin{array}{c}\text { Total } \\
\text { Mass } \\
\text { Recovered } \\
(\mathrm{g})\end{array}$ & $\begin{array}{c}\text { Recovery } \\
\text { to } \\
\text { Underflow } \\
(\mathrm{wt} \%)\end{array}$ & $\begin{array}{c}\text { Experimental } \\
\text { Losses } \\
(\mathrm{wt} \%)\end{array}$ \\
\hline 50 & 1.75 & 50 & 0.0088 & 49.1 & 36.2 & 2.6 & 10.4 & 49.2 & 21.14 & -0.20 \\
\hline 50 & 1.75 & 85 & 0.0143 & 47.7 & 30.5 & 3.5 & 12.2 & 46.2 & 26.41 & 3.14 \\
\hline
\end{tabular}

14. Recovery of $21-54 \mu \mathrm{m}\left(\mathrm{d}_{50}=34 \mu \mathrm{m}\right)$ cleaned silica with reverse osmosis (RO) water

\begin{tabular}{|c|c|c|c|c|c|c|c|c|c|c|}
\hline $\begin{array}{c}\text { Air } \\
\text { Flowrate } \\
(\%)\end{array}$ & $\begin{array}{c}\text { Water } \\
\text { Flowrate } \\
(\%)\end{array}$ & $\begin{array}{c}\text { Water } \\
\text { Volumetric } \\
\text { Rate } \\
(\mathrm{L} / \mathrm{min})\end{array}$ & $\begin{array}{c}\text { Water } \\
\text { Superficial } \\
\text { Velocity } \\
(\mathrm{m} / \mathrm{s})\end{array}$ & $\begin{array}{c}\text { Total } \\
\text { Feed } \\
(\mathrm{g})\end{array}$ & $\begin{array}{c}\text { Mas in } \\
\text { Cyclone } \\
(\mathrm{g})\end{array}$ & $\begin{array}{c}\text { Mass in } \\
2^{\text {nd }} \\
\text { Chamber } \\
(\mathrm{g})\end{array}$ & $\begin{array}{c}\text { Underflow } \\
\text { Mass } \\
(\mathrm{g})\end{array}$ & $\begin{array}{c}\text { Total } \\
\text { Mass } \\
\text { Recovered } \\
(\mathrm{g})\end{array}$ & $\begin{array}{c}\text { Recovery } \\
\text { to } \\
\text { Underflow } \\
(\mathrm{wt} \%)\end{array}$ & $\begin{array}{c}\text { Experimental } \\
\text { Losses } \\
(\mathrm{wt} \%)\end{array}$ \\
\hline 40 & 30 & 0.65 & 0.0057 & 49.8 & 30.8 & 5.4 & 10.1 & 46.3 & 21.81 & 7.03 \\
\hline 40 & 50 & 1.00 & 0.0088 & 49.9 & 36.6 & 2.7 & 10.6 & 49.9 & 21.24 & 0.00 \\
\hline 40 & 70 & 1.35 & 0.0118 & 49.9 & 31.5 & 4 & 12.9 & 48.4 & 26.65 & 3.01 \\
\hline 40 & 70 & 1.35 & 0.0118 & 49.8 & 19.8 & 7.5 & 13.9 & 41.2 & 33.74 & 17.21 \\
\hline 40 & 85 & 1.63 & 0.0143 & 49.9 & 26.7 & 5.9 & 14.9 & 47.5 & 31.37 & 4.81 \\
\hline
\end{tabular}

15. Recovery of $21-54 \mu \mathrm{m}\left(\mathrm{d}_{50}=34 \mu \mathrm{m}\right)$ cleaned silica with tap water Same as Table 6 -Recovery of cleaned rough silica flour $21-54 \mu \mathrm{m}\left(\mathrm{d}_{50}=34\right.$ $\mu \mathrm{m})$ 
16. Recovery of $21-54 \mu \mathrm{m}\left(\mathrm{d}_{50}=34 \mu \mathrm{m}\right)$ cleaned silica particles with different salt solution (Aluminium sulphate) concentrations

\begin{tabular}{|c|c|c|c|c|c|c|c|c|c|c|}
\hline $\begin{array}{c}\text { Salt } \\
\begin{array}{c}\text { Concentrati } \\
\text { on } \\
(\mathrm{mg} / \mathrm{L})\end{array}\end{array}$ & $\begin{array}{c}\text { Conductivi } \\
\text { ty } \\
(\mu \mathrm{S})\end{array}$ & $\begin{array}{c}\text { Air } \\
\text { Flowr } \\
\text { ate } \\
(\%)\end{array}$ & $\begin{array}{c}\text { Water } \\
\text { Superficial } \\
\text { Velocity } \\
(\mathrm{m} / \mathrm{s})\end{array}$ & $\begin{array}{c}\text { Total } \\
\text { Feed } \\
(\mathrm{g})\end{array}$ & $\begin{array}{c}\text { Mas in } \\
\text { Cyclon } \\
\mathrm{e} \\
(\mathrm{g})\end{array}$ & $\begin{array}{c}\text { Mass in } \\
2^{\text {nd }} \\
\text { Chambe } \\
\mathrm{r} \\
(\mathrm{g})\end{array}$ & $\begin{array}{c}\text { Underflo } \\
\mathrm{w} \\
\text { Mass } \\
(\mathrm{g})\end{array}$ & $\begin{array}{c}\text { Total } \\
\text { Mass } \\
\text { Recovere } \\
\mathrm{d} \\
(\mathrm{g})\end{array}$ & $\begin{array}{c}\text { Recovery } \\
\text { to } \\
\text { Underflo } \\
\mathrm{w} \\
(\mathrm{wt} \%)\end{array}$ & $\begin{array}{c}\text { Experiment } \\
\text { al Losses } \\
(\mathrm{wt} \%)\end{array}$ \\
\hline 50 & 247 & 40 & 0.0143 & 49.8 & 27.9 & 3.9 & 13.9 & 45.7 & 30.42 & 8.23 \\
\hline 400 & 497 & 40 & 0.0143 & 49.6 & 26.1 & 6.1 & 16.2 & 48.4 & 33.47 & 2.42 \\
\hline 800 & 637 & 40 & 0.0143 & 49.9 & 33 & 1.1 & 15.9 & 50 & 31.8 & -0.20 \\
\hline 1000 & 703 & 40 & 0.0143 & 50 & 30.8 & 2.4 & 15.8 & 49 & 32.24 & 2.00 \\
\hline 2000 & 966 & 40 & 0.0143 & 48.9 & 28.4 & 2.2 & 16.6 & 47.2 & 35.17 & 3.48 \\
\hline
\end{tabular}

* Highlighted results have being omitted from the results presented in the Chapter 6 as they are above the standard experimental loss. 


\section{APPENDIX E}

\section{Underflow Recovery for Different Particles at a Constant Water Flux.}

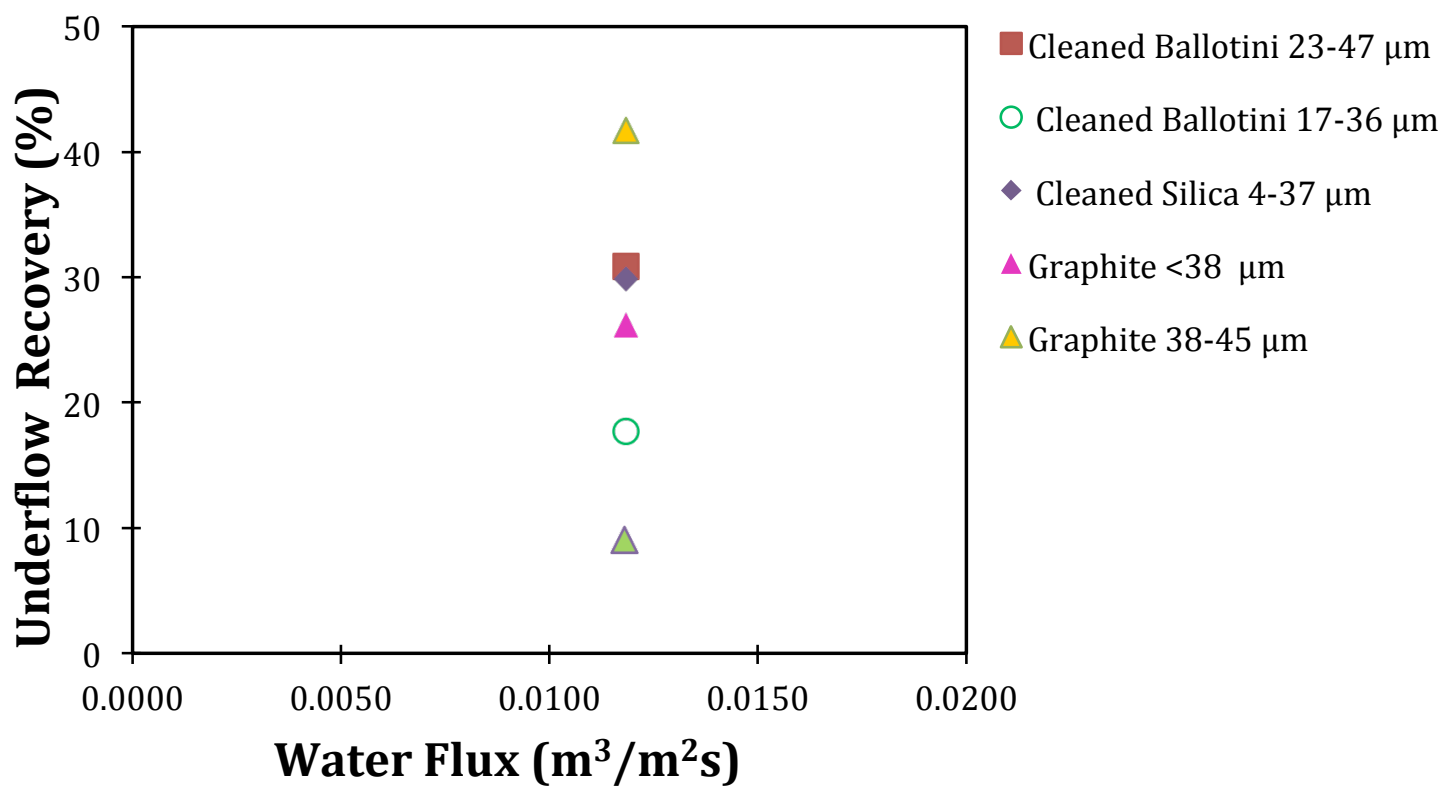

Figure E.1: Underflow recoveries for different particle types at $0.01 \mathrm{~m} / \mathrm{s}$ water flux. 


\section{Underflow Recovery for Esterified (Hydrophobised) and As Received (Uncleaned) Glass Ballotini}

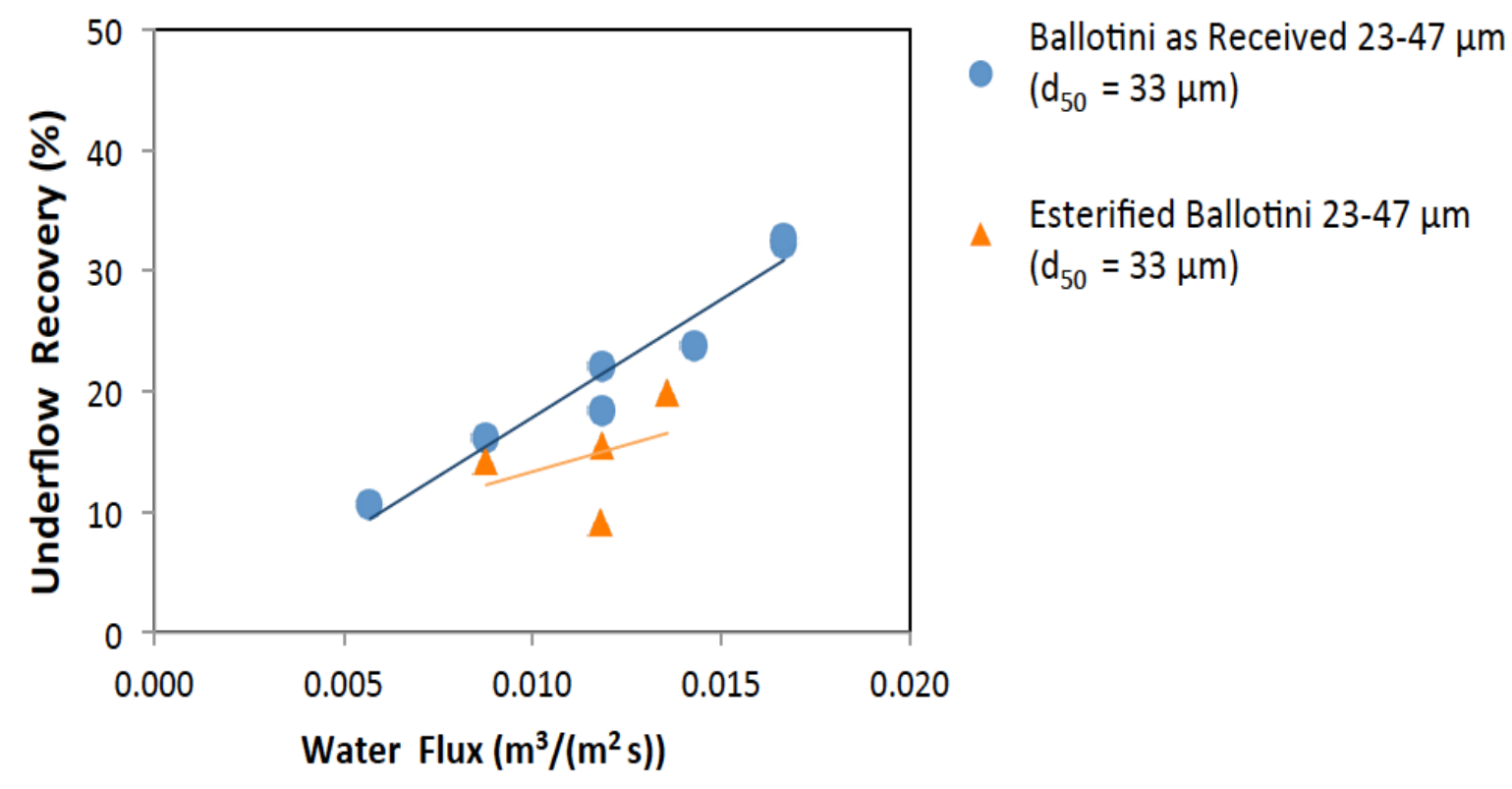

Figure E.2: Underflow recovery for Esterified (Hydrophobised) and As Received (Uncleaned) glass ballotini in $23-47 \mu \mathrm{m}$ size range. 


\section{APPENDIX F}

\section{Audit of Experimental Losses}

\begin{tabular}{|c|c|c|c|c|}
\hline 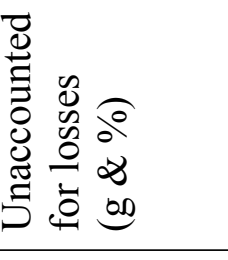 & $\div 8^{\circ}$ & $=\stackrel{0}{\overbrace{}^{\circ}}$ & $\infty \stackrel{\circ}{\circ}$ & $\sim \underset{0}{\stackrel{0}{+}}$ \\
\hline 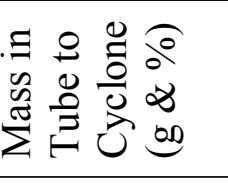 & $\because$ 事 & ? & $\ddot{m}$ & $\stackrel{n}{\rightarrow} \stackrel{\circ}{\circ}$ \\
\hline 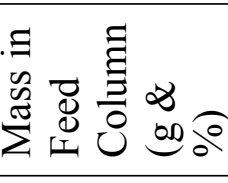 & : & $2 \stackrel{0}{\circ} \stackrel{0}{0}^{\circ}$ & $\because \stackrel{0}{\circ}$ & $=-\frac{8}{0}$ \\
\hline 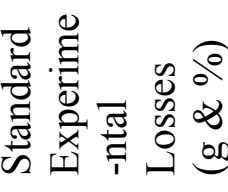 & 굴 & 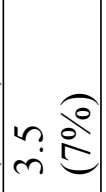 & $+\stackrel{\circ}{\circ}$ & $=$ \\
\hline 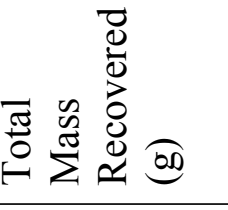 & $\stackrel{+}{\ddot{2}}$ & $\begin{array}{l}3 \\
6 \\
6 \\
q\end{array}$ & 字 & $\vec{\infty}$ \\
\hline 离 古 & $\vec{m}$ & గె & in & $\dot{m}$ \\
\hline 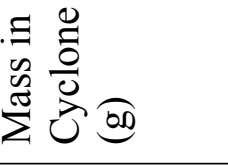 & $?$ & $\vec{q}$ & $\ddot{\dot{m}}$ & $\stackrel{+}{\dot{f}}$ \\
\hline 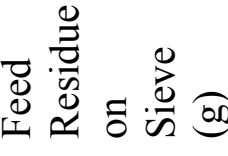 & $\stackrel{t}{2}$ & $\tilde{o}$ & $\hat{o}$ & 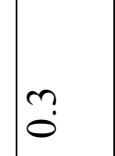 \\
\hline 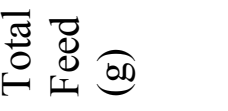 & 凩 & in & in & 으 \\
\hline 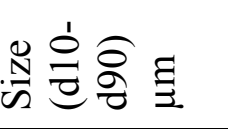 & $\hat{\tilde{g}}$ & 莡 & 总 & 守 \\
\hline 莺 & 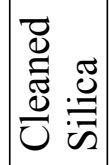 & 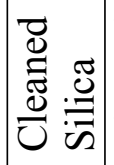 & 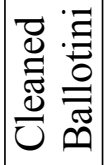 & 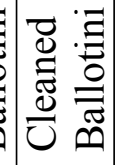 \\
\hline
\end{tabular}




\section{APPENDIX G}

\section{Raw Data of Electrostatic Experiments}

Due to high volume of data in Figure 7.8, 7.11, 7.12 and 7.14 these are included in the enclosed CD. 